\title{
A REVIEW OF EXTANT CROATIAN FRESHWATER FISH AND LAMPREYS Annotated list and distribution
}

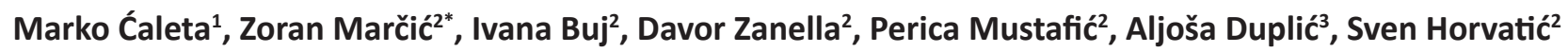 \\ ${ }^{1}$ Faculty of Teacher Education, Savska cesta 77, University of Zagreb, Zagreb, Croatia \\ ${ }^{2}$ Faculty of Science, Department of Zoology, Rooseveltov trg 6, University of Zagreb, Zagreb, Croatia \\ ${ }^{3}$ Ministry of Environmental Protection and Energy, Radnička cesta 80, Zagreb, Croatia \\ *Corresponding Author, Email: zoran.marcic@biol.pmf.hr
}

\section{ARTICLE INFO}

Received: 22 July 2019

Accepted: 26 September 2019

\section{Keywords:}

Danube drainage

Adriatic basin

Endemism

Introductions

Translocations

How to Cite

\section{ABSTRACT}

A checklist of the freshwater fish fauna of Croatia is presented for the first time. It is based on 1360 publications of historical and recent data in the literature. According to the literature review, there were 137 fish species in 30 families and 75 genera recorded in Croatia. The checklist is systematically arranged and provides distributional data of the freshwater fish fauna as well as whether the species is endemic, introduced or translocated.

\section{INTRODUCTION}

The Republic of Croatia is a small country with a land area of $56,594 \mathrm{~km}^{2}$ at the crossroads of several European biogeographical regions: Continental, Alpine, Mediterranean and, on its north-east, fragments of the Panonian biogeographical region are present (EEA, 2002). Croatia's geographical position includes two river basins (Adriatic Sea and Black Sea basins) with an interesting geological history that has resulted in one of the most diverse freshwater ichthyofaunas in Europe. With 137 species that are strictly freshwater or occasionally use freshwater, Croatia has among the highest species richness in Europe (Mrakovčić et al., 2006). In particular, the Dinaric karst region of Croatia (Banarescu, 2004; Smith and Darwall, 2006; Oikonomou et al., 2014) harbours numerous endemic species that are found only in Croatia, while some are also found in neighbouring Bosnia and Herzegovina (Ćaleta et al., 2015). Despite the long tradition of ichthyology in these parts through the $19^{\text {th }}$ and $20^{\text {th }}$ century (most important researchers were Johann Jakob Heckel, Rudolf Kner, Franz Steindachner, Luka Trgovčević, Mijo Kišpatić, Josipa Plančić, Tihomir Vuković, Dobrila Habeković, Milorad Mrakovčić i Tomislav Treer), in the $21^{\text {st }}$ century, new species are still being discovered and described (Kovačić, 2005; Freyhof and Stelbrink, 2007; Mustafić et al., 2008; Miller, 2009; Bogutskaya and Zupančič, 2010; Buj et al., 2010; Marčić et 
al., 2011; Bogutskaya et al., 2012; Tutman et al., 2017). To date, the only comprehensive list of the freshwater fishes of Croatia was compiled within the freshwater fish fauna of Yugoslavia (Taler, 1953; Vuković and Ivanović, 1971), which ceased to exist as a state in 1991, breaking up into several sovereign states lying in different biogeographical regions. Despite more than a century of ichthyological research, no checklist has ever been compiled of the Croatian freshwater ichthyofauna. Previous publications about the freshwater fishes of Croatia were aimed at threatened (Mrakovčić et al., 2006) or endemic (Ćaleta et al., 2015) fishes. Furthermore, to date, the literature data on the freshwater fish of Croatia have not been comprehensively collected, analysed and published.

The objective of this review article was to collect and analyse all the available literature data on the freshwater fish of Croatia, and to draft an annotated checklist of the species currently found in Croatia.

\section{MATERIAL AND METHODS}

This is the first annotated checklist of the freshwater fish species of Croatia, which includes a compilation of the available literature for Croatia. The bibliography includes scientific papers, professional papers, doctoral dissertations, master theses, books, monographs, chapters in books and technical reports. Grey literature like unpublished reports, professional papers, diploma theses and government documents were not analyzed for the purposes of this checklist. In addition to the exclusively freshwater species, data are also provided on euryhaline species that inhabit brackish and transitional waters (Aphanius fasciatus, Alosa fallax, Platichthys flesus, Dicentrarchus labrax, Sparus aurata, Gobiids and Mugilids) and migratory fish species (Anguilla anguilla, Petromyzon marinus and Acipenserids). In the text of article were used scientific species names while in the species list were listed valid English names (Froese and Pauly, 2019), authority and Croatian names. The list was compiled on the basis of the latest taxonomic classifications of orders, families, genera and species (Stout et al., 2016; Eschmeyer et al., 2018a; Eschmeyer et al., 2018b; Schonhuth et al., 2018; Tan and Armbruster, 2018). Within each family, the genera are listed in alphabetical order. The checklist includes a distribution range for each species, and data as to whether it is an endemic, introduced or translocated species. The list also includes species that are considered regionally extinct (EX in the list) and unsuccessfully introduced species (UI in the list).

The border between the Danube drainage and the Adriatic Sea basin passes through the Lika area, which is located in the Alpine biogeographic region (Fig 1). Except the rivers, some karst fields also belong to different river drainages/ basins. Thus, the Danube drainage includes the following fields: Lug, Jasenačko, Drežničko, Stajničko (Mrežnica River drainage) and Krbavsko (Una River Drainage); which are inhabited by the endemic species of the genus Telestes and Delminichthys.

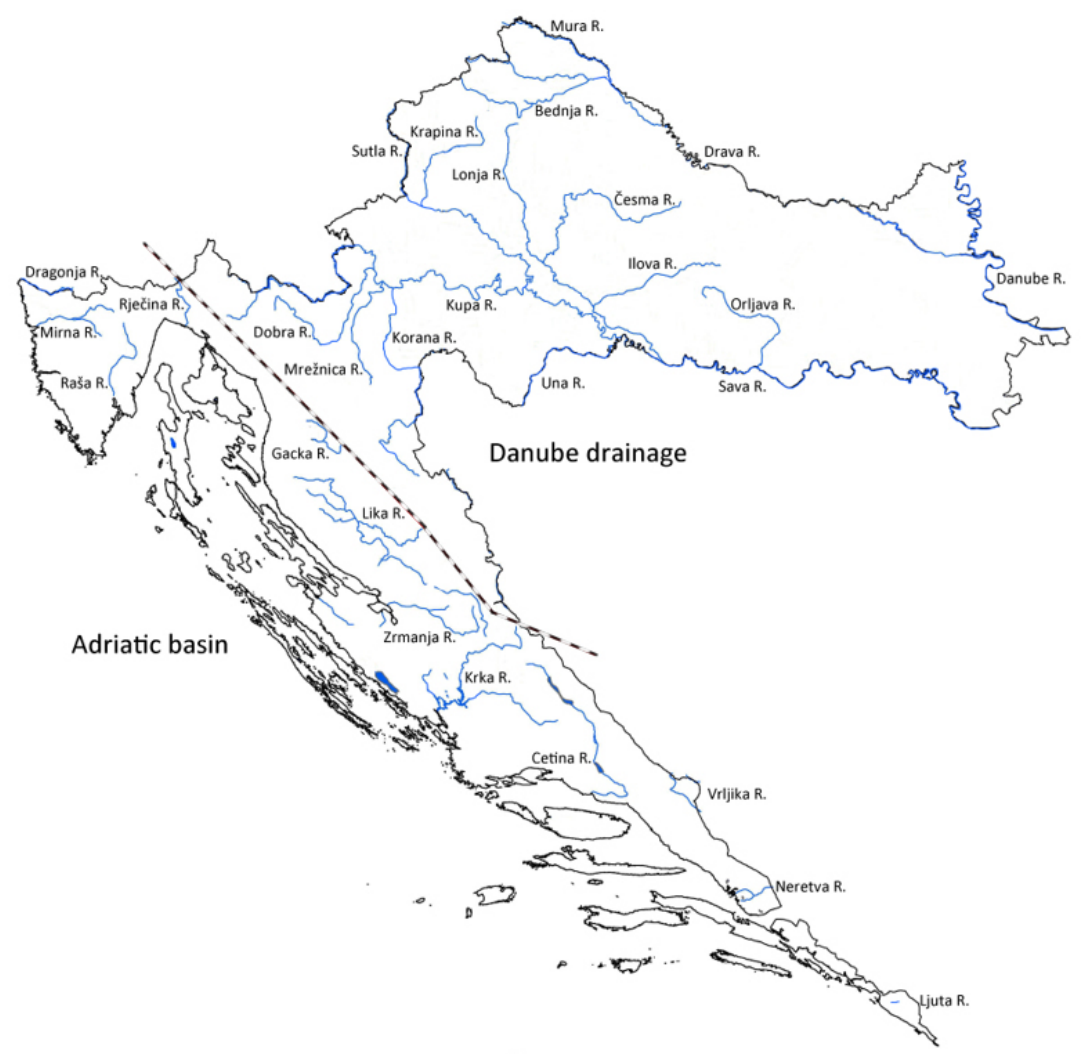

Fig 1. Map of Croatia with two drainages (basins) and major river courses (dashed line represents the basin boundary) 


\section{RESULTS AND DISCUSSION}

\section{Croatian ichthyofauna bibliography}

For the purposes of checklist, available literature data on the freshwater fishes of Croatia were collected. This included 1360 publications in the period since 1771, and represents the most comprehensive Croatian ichthyofauna bibliography.

The number of literature reports varies largely depending on the historical period. There are only 5 publications from the $18^{\text {th }}$ century, and 153 in the $19^{\text {th }}$ century. A total of 775 publications date back to the $20^{\text {th }}$ century, while in the first 19 years of the $21^{\text {st }}$ century, there have been 424 publications. A review of the number of literature reports by species shows that the most frequently mentions are those common and recognisable species used in human nutrition, such as Salmo trutta, Cyprinus carpio, Anguilla anguilla, Esox lucius and Silurus glanis. A total of 25 fish species are mentioned in more than 100 literature reports (in checklist (Appendix 1) species no. 5, 6, 22, 25, 26, 27, $28,34,36,57,61,64,66,82,86,89,93,94,98,99,101$, $126,124,131,132)$. However, there are species for which there are very few literature reports, with six species mentioned in fewer than 10 publications (in checklist (Appendix 1) species no. 17, 30, 39, 58, 103, 106). These are mostly species that have been recently described, discovered or whose taxonomic status has only recently been resolved, and therefore have not been discussed in the literature.

\section{Diversity of the Croatian ichthyofauna}

Based on the collected data, the freshwater ichthyofauna of Croatia currently totals 137 species (Table 1; Appendix $1)$, for which literature records are provided. These are species that are native, endemic and succesfully introduced. The freshwater ichthyofauna of Croatia can be classified into two classes, 16 orders, 30 families and 75 genera. The greatest diversity is found within the order Cypriniformes (78 species, $56.9 \%$ ), followed by the orders Perciformes (13 species, 9.5\%), Gobiiformes and Salmoniformes (11 species, 8.0\%) (Table 1). At the family level, the highest species richness is found amongst the Leuciscidae (48 species, 35.0\%), Gobiidae and Salmonidae (11 species, 8.0\%), Cobitidae (10 species, 7.3\%) and Percidae and Cyprinidae (8 species, 5.8\%). All other families are represented by six or fewer species (Table 1 ).

Several explanations have been posed for the exceptionally high ichthyodiversity in this relatively small area. First, Croatian freshwaters are divided into two major drainage basins and their rivers and lakes have different geographical, hydrological and ecological features. Most of the rivers belonging to Black Sea Basin (also called the Danube drainage system, therefore hereinafter:
Danube drainage) are long, with many tributaries and many are interconnected. On the other hand, the rivers of the Adriatic Sea Basin (hereinafter: Adriatic Basin) are much shorter and isolated from each other, with few or no tributaries, thus spurring allopatric speciation and resulting in very high species richness and high degree of endemism (Marčić et al., 2011; Buj et al., 2014; Buj et al., 2015; Ćaleta et al., 2015; Buj et al., 2017; Buj et al., 2019 in press). Moreover, $50 \%$ of the Croatian territory is covered by karst, which is a geologic and geographic feature that favoured development of diverse, often isolated water habitats. As a specific feature of this area, presence of the karst fields (known as 'polje') can be mentioned. Those are karst relief forms where surface flows sink underground in contact with limestone. Isolated, unique habitats with specific hydrological conditions are often inhabited by numerous endemic and endangered species. The second important reason causing the origin of a rich and diverse fish community lies in the complex geological history of the region, which included various vicariant events and periods of favourable conditions, promoting diversification and unconstrained evolutionary history of species and genera. This situation is evident in the recently described evolutionary history of the genus Telestes (Buj et al., 2017), and spined loaches of the genus Cobitis (Buj et al., 2015), though it is likely also true for other freshwater fishes whose evolutionary history is yet to be revealed. Moreover, the Dinaric karst region in Croatia seems to harbour ancient populations of certain freshwater species (Gasterosteus) and genera (e.g. Telestes, Delminichthys, Phoxinellus), and represents the site from where colonization of a wider area begun, whilst still maintaining reservoirs of ancient genetic diversity (Buj et al., 2017; Buj et al., 2019 in press).

In addition to the extraordinary high species diversity, there are natural hybrid biotypes that originated through the hybridization of two species in geological periods when temporary connections were formed between rivers. Such past geologic hybridizations left traces in the recent genetic composition of several populations, whose genomes are comprised of alleles belonging to two or more species. Hybrid biotypes of $C$. elongatoides (all female triploids) have been reported from the Danube River drainage in Croatia (Buj et al., 2008), as well as introgressions in some populations of $S$. obtusirostris (Sušnik et al., 2007; Razpet et al., 2007). Furthermore, recent investigations (unpublished data) have revealed natural interspecific and even intergeneric hybrids in certain Squalius populations Buj et al. 2019 in press).

A total of 49 species of freshwater fish in Croatia are endemic species. However, the Danube drainage is inhabited by 9 endemic species (Eudontomyzon vladykovi, Cobitis elongata, Gobio obtusirostris, R. uranoscopus, Romanogobio vladykovi, Alburnus sava, Rutilus virgo, Hucho hucho, Gymnocephalus schraetser) which have wider distribution (endemic to the Danube basin). 
Table 1. Taxonomic distribution, species number, number of alien and number of endemic species of freshwater fishes of Croatia ( $*$ without Danube drainage endemics)

\begin{tabular}{|c|c|c|c|c|c|c|}
\hline ORDER & FAMILY & GENERA & SPECIES & $\%$ species & ALIEN & TRUE ENDEMIC* \\
\hline PETROMYZONTIFORMES & PETROMYZONTIDAE & 3 & 3 & $2,19 \%$ & - & 1 \\
\hline ACIPENSERIFORMES & ACIPENSERIDAE & 1 & 2 & $1,46 \%$ & - & - \\
\hline ANGUILLIFORMES & ANGUILLIDAE & 1 & 1 & $0,73 \%$ & - & - \\
\hline \multirow[t]{5}{*}{ CLUPEIFORMES } & CLUPEIDAE & 1 & 1 & $0,73 \%$ & - & - \\
\hline & COBITIDAE & 3 & 10 & $7,30 \%$ & - & 4 \\
\hline & NEMACHEILIDAE & 1 & 1 & $0,73 \%$ & - & - \\
\hline & ACHEILOGNATHIDAE & 1 & 1 & $0,73 \%$ & - & - \\
\hline & CYPRINIDAE & 4 & 8 & $5,84 \%$ & 2 & 2 \\
\hline \multirow{5}{*}{ CYPRINIFORMES } & GOBIONIDAE & 3 & 6 & $4,38 \%$ & 1 & 1 \\
\hline & LEUCISCIDAE & 17 & 48 & $35,04 \%$ & - & 21 \\
\hline & TINCIDAE & 1 & 1 & $0,73 \%$ & - & - \\
\hline & XENOCYPRIDIDAE & 2 & 3 & $2,19 \%$ & 3 & - \\
\hline & SILURIDAE & 1 & 1 & $0,73 \%$ & - & - \\
\hline \multirow{2}{*}{ SILURIFORMES } & ICTALURIDAE & 1 & 2 & $1,46 \%$ & 2 & - \\
\hline & ESOCIDAE & 1 & 1 & $0,73 \%$ & - & - \\
\hline ESOCIFORMES & UMBRIDAE & 1 & 1 & $0,73 \%$ & - & - \\
\hline SALMONIFORMES & SALMONIDAE & 6 & 11 & $8,03 \%$ & 4 & 1 \\
\hline GADIFORMES & LOTIDAE & 1 & 1 & $0,73 \%$ & - & - \\
\hline GOBIIFORMES & GOBIIDAE & 8 & 11 & $8,03 \%$ & 4 & 6 \\
\hline PLEURONECTIFORMES & PLEURONECTIDAE & 1 & 1 & $0,73 \%$ & - & - \\
\hline \multirow{2}{*}{ CYPRINODONTIFORMES } & CYPRINODONTIDAE & 1 & 1 & $0,73 \%$ & - & - \\
\hline & POECILIIDAE & 1 & 1 & $0,73 \%$ & 1 & - \\
\hline MUGILIFORMES & MUGILIDAE & 4 & 6 & $4,38 \%$ & - & - \\
\hline BLENNIFORMES & BLENNIIDAE & 1 & 1 & $0,73 \%$ & - & - \\
\hline \multirow[t]{3}{*}{ SCORPAENIFORMES } & COTTIDAE & 1 & 1 & $0,73 \%$ & - & - \\
\hline & MORONIDAE & 1 & 1 & $0,73 \%$ & - & - \\
\hline & CENTRARCHIDAE & 2 & 2 & $1,46 \%$ & 2 & - \\
\hline \multirow[t]{3}{*}{ PERCIFORMES } & PERCIDAE & 4 & 8 & $5,84 \%$ & - & - \\
\hline & SPARIDAE & 1 & 1 & $0,73 \%$ & - & - \\
\hline & GASTEROSTEIDAE & 1 & 1 & $0,73 \%$ & - & - \\
\hline 16 & 30 & 75 & 137 & $100 \%$ & 19 & 36 \\
\hline
\end{tabular}


The remaining 40 species ( $29.2 \%$ of the total ichthyofauna) are true endemic species (narrower distribution) to Croatia and/or to Adriatic basin (Table 1). Gobio obtusirostris inhabits both drainages/basins because it has been translocated to the Adriatic basin. The true endemic species belong to seven families, with 21 endemic species included in the family Leuciscidae, or $15.3 \%$ of all recorded species (Ćaleta et al., 2015). Among the true endemics there are also 15 species (10.9\% of the total ichthyofauna) exclusively inhabiting the waters of Croatia (Cobitis dalmatina, C. jadovaensis, Delminichthys jadovensis, D. krbavensis, Phoxinellus dalmaticus, Squalius illyricus, $S$. zrmanjae, Telestes croaticus, T. fontinalis, T. karsticus, T. miloradi, T. polylepis, T. turskyi, T. ukliva and Knipowitschia mrakovcici) (stenoendemic species). Considering that the historical literature data were consulted, the checklist also includes (though not enumerated) those species that formerly inhabited the freshwaters of Croatia, and which are today considered regionally extinct (genus Acipenser and Huso and the Alosa immaculata) (Table 2).

To date, according to literature data, a total of 32 freshwater fish species have been introduced to Croatia (Piria et al., 2018; Pofuk et al., 2017.; personal data). The freshwaters of Croatia have 19 alien species (naturalised and acclimatized), introduced from other geographic areas and continents (Table 1). Four of them have been introduced into the Danube drainage, and two into the Adriatic Basin. The remaining 13 species have been introduced into both basins (Piria et al., 2018). The checklist of Croatian freshwater fish species lists (though not enumerated) exotic (alien) (unsuccessfully introduced) species (total of
13) which were introduced historically or escaped (from aquaculture or aquaria) and were reported in the waters of Croatia but failed to create self-sustaining populations (Table 2). In addition to the introduced species, there are also species that are native to the Republic of Croatia, but have been translocated between the basins. There are 17 species, native to the Danube drainage, known to have been introduced (naturalised and acclimatized) into the Adriatic Basin (Pofuk et al., 2017.; personal data). The only recorded species translocated from Adriatic Basin to the Danube drainage is Gasterosteus aculeatus.

\section{Geographical distribution of the freshwater fish diversity (hot spots)}

In the Danube drainage in Croatia (total area $35132 \mathrm{~km}^{2}$ ) 81 freshwater fish species are present, of which 64 species are native and the remaining 17 are alien. In the Adriatic Basin (total area $21405 \mathrm{~km}^{2}$ ) were recorded 90 fish species of which 60 species are native while 30 species are introduced from Danube drainage (17 species) or alien (15 species). Denotation of the waters of the Adriatic Basin in Croatia as the ichthyodiversity 'hot spot' is clearly argumented by the exceptionaly high number of fish species distributed there, and particularly high number of endemic fishes (36 species) (Table 3). On the other hand, rivers of the Danube drainage harbour a greater number of species, which is expected, due to their greater length and interconnections. Rivers with the highest number of species in Croatia are Drava, Sava, Kupa, Mura, Una

Table 2. Taxonomic distribution, number of extinct (EX) and number of unsuccessfully introduced (UI) species in Croatia

\begin{tabular}{|c|c|c|c|c|c|}
\hline ORDER & FAMILY & GENERA & SPECIES & $E X$ & UI \\
\hline \multirow{3}{*}{ ACIPENSERIFORMES } & ACIPENSERIDAE & 2 & 6 & 5 & 1 \\
\hline & & & & & \\
\hline & POLYODONTIDAE & 1 & 1 & - & 1 \\
\hline CHARACIFORMES & SERRASALMIDAE & 1 & 1 & - & 1 \\
\hline CICHLIFORMES & CICHLIDAE & 1 & 1 & - & 1 \\
\hline CLUPEIFORMES & CLUPEIDAE & 1 & 1 & 1 & - \\
\hline GOBIIFORMES & ODONTOBUTIDAE & 1 & 1 & - & 1 \\
\hline PERCIFORMES & MORONIDAE & 1 & 1 & - & 1 \\
\hline \multirow[t]{2}{*}{ SALMONIFORMES } & SALMONIDAE & 3 & 4 & - & 4 \\
\hline & MOCHOKIDAE & 1 & 1 & - & 1 \\
\hline \multirow[t]{2}{*}{ SILURIFORMES } & ICTALURIDAE & 1 & 1 & - & 1 \\
\hline & CLARIIDAE & 1 & 1 & - & 1 \\
\hline 8 & 10 & 14 & 19 & 6 & 13 \\
\hline
\end{tabular}


Table 3. Distribution of fish taxa in Croatia by drainages (*without Danube drainage endemics; in brackets total number with species of brackish and transitional waters; bold - Danube drainage, italic - Adriatic Basin)

\begin{tabular}{|c|c|c|c|c|c|c|}
\hline Drainage & Orders & Families & Genera & Species & Introduced & True endemic* \\
\hline Mura & 9 & 20 & 44 & 57 & 10 & \\
\hline Drava & 11 & 22 & 51 & 70 & 15 & \\
\hline Bednja & 8 & 16 & 34 & 40 & 6 & \\
\hline Danube & 9 & 18 & 41 & 55 & 13 & \\
\hline Sava & 10 & 20 & 49 & 68 & 13 & \\
\hline Orljava & 7 & 15 & 32 & 38 & 6 & \\
\hline Una & 10 & 18 & 43 & 57 & 8 & 2 \\
\hline llova & 7 & 16 & 36 & 44 & 9 & \\
\hline Česma & 7 & 16 & 35 & 43 & 9 & \\
\hline Lonja & 7 & 15 & 30 & 36 & 5 & \\
\hline Krapina & 8 & 16 & 33 & 39 & 5 & \\
\hline Sutla & 8 & 16 & 30 & 38 & 4 & \\
\hline Kupa & 10 & 19 & 45 & 59 & 12 & \\
\hline Dobra & 7 & 15 & 32 & 38 & 5 & \\
\hline Mrežnica & 7 & 15 & 31 & 37 & 5 & 2 \\
\hline Korana & 8 & 17 & 34 & 41 & 8 & \\
\hline Dragonja & 5 & 7 & 10 & $5(12)$ & 1 & 1 \\
\hline Mirna & 10 & 17 & 28 & 19 (31) & 10 & 6 \\
\hline Raša & 8 & 11 & 17 & $9(19)$ & 3 & 4 \\
\hline Rječina & 5 & 6 & 8 & $3(10)$ & & \\
\hline Gacka & 4 & 7 & 9 & 9 & 8 & \\
\hline Lika & 5 & 9 & 17 & 17 & 12 & 3 \\
\hline Zrmanja & 10 & 15 & 23 & 15 (25) & 5 & 4 \\
\hline Krka & 11 & 18 & 30 & 22 (33) & 11 & 9 \\
\hline Cetina & 11 & 18 & 29 & 22 (33) & 12 & 8 \\
\hline Vrljika & 4 & 7 & 10 & 10 & 5 & 5 \\
\hline Neretva & 13 & 20 & 36 & 31 (43) & 12 & 14 \\
\hline Ljuta & 2 & 2 & 5 & 5 & 1 & 2 \\
\hline
\end{tabular}

and Danube, all of them contained inside the Danube drainage. Rivers with the highest number of species inside the Adriatic Basin are Neretva, Krka, Cetina and Mirna (Figure 1). Some of those species (particularly in the Mirna River) are also brackish fish species (Mugilids) that regularly enter river mouths and the lower stretches of rivers. 


\section{Implications for future study}

The checklist presented here clearly displays the exceptional diversity of the Croatian ichthyofauna. Research conducted in recent decades has revealed the great diversity of Croatian freshwater fishes at several levels: species diversity, intraspecific genetic diversity, natural hybrid richness and functional diversity of freshwater ecosystems (Buj et al., 2008; Buj et al., 2014; Buj et al., 2018; Buj et al., 2019 in press). On the other hand, drawbacks have been recognised with regard to the current knowledge and areas where information is scarce. There are still many outstanding questions, which these can serve as guidelines for future study. The salmonids are an example of an important case where the taxonomy is yet to be resolved (Kottelat and Freyhof 2007). The unresolved taxonomy of the genus Salmo is a problem not only for Croatia but is characteristic for all of Europe (Kottelat and Freyhof 2007). A debate is still ongoing as to whether there is a single widespread trout species with pronounced intraspecific structuring (at least five lineages) that inhabits most European waters, or whether these are several different species, and no final answer has yet been reached (Bernatchez et al., 1992; Kottelat and Freyhof, 2007; Schenekar et al., 2014). Moreover, the taxonomic status and validity of several species described as endemic to Adriatic Basin (e.g. Salmo visovacensis, S. dentex) is questionable (see Kottelat and Freyhof, 2007). Due to their importance in freshwater ecosystems, but also in fisheries, determining the exact taxonomic status of trout populations in Croatia, as a prerequisite for their adequate conservation, should be the focus of further scientific study. The possibility of the existence of cryptic diversity has already been reported for genus Phoxinus (Palandačić et al., 2015). The possible discovery of an endemic species would be very important in the conservation sense. A specific taxonomic problem is also seen in the family Petromizontidae (Tutman et al., 2017) since its taxonomy is unsettled. Comprehensive taxonomic study, including detailed analysis of the morphology, and also multilocus phylogenetic research is required to resolve this problem. Moreover, inclusion of both morphological characters and phylogenetic relationships in taxonomical studies is a necessary prerequisite to resolve taxonomic dilemmas, instead of the exclusive reliance on morphological characters which in the past has led to many incorrect taxonomic conclusions.

In addition to resolving taxonomic uncertainties, an important direction for future research would be to confirm the native distribution ranges for several species (e.g. Petromyzontidae species, Alosa fallax, Chondrostoma phoxinus, Rutilus spp., Romanogobio spp., Misgurnus fossilis). Due to contradictory reports by earlier authors (Langhoffer, 1904; Trgovčević, 1932; Rössler, 1931; Taler, 1953; Basioli, 1957a; Leiner \& Popović, 1984; Leiner, $1985)$, it is often not clear whether a report of a certain species at a locality outside its common distribution range is due to misidentification (Barbatula barbatula in the Lika River, Barbus barbus in the Neretva River, Cobitis taenia in the Cetina River, Leuciscus souffia in the Cetina River), or whether is it a case of a rare or locally extinct species, if there are no recent reports (Rutilus aula in the Rječina River and in the Vransko Lake, Scardinius hesperidicus in the Istria, Salmo dentex in the Cetina River). In some cases, it is easy to decide between the two hypotheses, while in others only extensive field studies can enable exclusion of one of the hypotheses.

Finally, future research should also focus on specific populations of well-known species, for which there is a lack of data on their status and they may be locally extinct or present only in very low population densities (e.g. Salmo obtusirostris in the Krka River, Leucapius delineates in the Sava River system). Due to growing pressures on freshwater communities, such studies are urgent and should be followed by adequate conservation measures to ensure the future viability and unconstrained evolution of the observed exceptionally rich ichthyodiversity.

Appendix 1. Checklist of Croatian ichthyofauna (numbers before species indicate that species is native, endemic or succesfuly introduced; UI unsuccessfully introduced; $\mathrm{EX}=$ extinct species)

\section{CLASS: PETROMYZONTI}

\subsection{ORDER: PETROMYZONTIFORMES}

\subsubsection{FAMILY: PETROMYZONTIDAE Bonaparte, 1831}

\section{EUDONTOMYZON Regan, 1911}

\section{Eudontomyzon vladykovi Oliva \& Zanandrea, 1959}

Names under which the taxon was mentioned: Ammocoetes branchialis Cuvier; Eudontomyzon danfordi Regan, 1911; Eudontomyzon danfordi vladykovi Oliva \& Zanandrea, 1959; Eudontomyzon mariae Oliva \& Zanandrea, 1959; Lampetra fluviatilis Berg, 1931; Lampetra planeri (Bloch, 1784); Petromyzon fluviatilis Linnaeus, 1758; Petromyzon planeri Bloch, 1784

English name: Vladykov's lamprey

Croatian name: dunavska paklara

Range in Croatia: Danube Drainage

Literature: Taube (1777); Heckel and Kner (1858); Jurinac (1880); Jurinac (1881); Jurinac (1882); Mojsisovics (1883); Jurinac (1884); Mojsisovics (1884); Glowacki (1885); Anonymous (1886); Glowacki (1896); Hirc (1897); Ćurčić (1910); Hirc (1911); Langhoffer (1915); Vutskits (1918); Zanandrea (1958); Tadić (1959); Oliva and Zanandrea (1959); Zanandrea (1959); Vuković and Ivanović (1971); Klašterka (1979); Delić (1993); Habeković et al. (1997); Kottelat (1997); Lelek (1987); Mrakovčić et al. (1996b); Honsig-Erlenburg et al. (1997); Povž et al. (1998); Holčík and Delić (2000); Majer (2001); Mustafić (2001); Bath 
(2003); Delić et al. (2003a); Mustafić (2005); Sallai and Kontos (2005); Kottelat and Freyhof (2007); Sallai and Mrakovčić (2007); Sallai and Kontos (2008); Zanella et al. (2008b); Delić et al. (2009); Dumbović et al. (2009); Jelić et al. (2009); Sofradžija (2009); Bučar et al. (2010); Buj (2010c); Perović and Tvrtković (2010); Tvrtković (2010); Jelić et al. (2012); Ćaleta et al. (2015); Piria et al. (2016b)

Remarks: endemic to the Danube drainage; literature reports of Eudontomyzon mariae are result of misidentification

\section{LAMPETRA Gray, 1851}

2. Lampetra soljani Tutman, Freyhof, Dulčić, Glamuzina \& Geiger, 2017

Names under which the taxon was mentioned: Eudontomyzon mariae Oliva \& Zanandrea, 1959; Lampetra fluviatilis Berg, 1931; Lampetra planeri (Bloch, 1784); Lampetra zanandreai Vladykov, 1955; Lethenteron zanandreai (Vladykov, 1955); Petromyzon fluviatilis Linnaeus, 1758

English name: Šoljan's brook lamprey

Croatian name: primorska paklara

Range in Croatia: Adriatic Basin - Neretva and Norin Rivers, Baćinska Lakes and Desne Lake

Literature: Maldini (1938a); Taler (1953a); Vuković and Ivanović (1971); Morović (1976); Sofradžija and Hadžiselimović (1981); Crivelli (1996); Mrakovčić et al. (1996a); Holčík and Mrakovčić (1997); Leiner (1998b); Tvrtković (1998); Mrakovčić et al. (2000a); Tvrtković and Franičević (2002); Zerunian (2004); Tvrtković and Veen (2006); Kottelat and Freyhof (2007); Glamuzina et al. (2010b); Povž (2011); Renaud (2011); Glamuzina et al. (2013); Ćaleta et al. (2015); Tutman et al. (2017)

Remarks: endemic to the Neretva River drainage and the Adriatic Basin, reports for the Istria are not confirmed

\section{PETROMYZON Linnaeus, 1758}

\section{Petromyzon marinus Linnaeus, 1758}

Names under which the taxon was mentioned: Petromyzon fluviatilis Linnaeus, 1758

English name: Sea lamprey

Croatian name: morska paklara

Range in Croatia: Adriatic Basin - Neretva River

Literature: Carrara (1846); Nardo (1847); Heckel and Kner (1858); Kolombatović (1881); Kolombatović (1882); Faber (1883); Katurić (1883); Kolombatović (1886a); Kolombatović (1886b); Kolombatović (1888); Carus (18891893); Botteri (1891); Kišpatić (1893); Damin (1900); Kosić (1903); Langhoffer (1904); Katurić 1907); Maldini (1938c); Taler (1953a); Zanandrea (1958); Tadić (1959); Vuković (1963); Morović (1965); Vuković and Ivanović (1971);
Sofradžija and Hadžiselimović (1981); Hardisty (1986); Kottelat (1997); Leiner (1998b); Holčík et al. (2004); Kottelat and Freyhof (2007); Jardas et al. (2008); Kovačić (2008); Tvrtković et al. (2009); Lipej and Dulčić (2010); Mrakovčić and Ćaleta (2011); Renaud (2011); Glamuzina et al. (2013)

Remarks: reports for the lower course and mouth of the Cetina River and Istria are not confirmed

\section{CLASS: ACTINOPTERI}

\subsection{ORDER: ACIPENSERIFORMES}

\subsubsection{FAMILY: ACIPENSERIDAE Bonaparte, 1831}

ACIPENSER Linnaeus, 1758

UI1 Acipenser baerii Brandt, 1869

English name: Siberian sturgeon

Croatian name: sibirska jesetra

Range in Croatia: Danube drainage - introduced locally

Remarks: non-native species; introduced to the Draganići fish farm for aquaculture purposes without a selfsustaining population

EX1 Acipenser gueldenstaedtii Brandt \& Ratzeburg, 1833

Names under which the taxon was mentioned: Acipenser aculeatus Lovetsky, 1834; Acipenser guldenstädti colchicus Marti, 1940

English name: Russian sturgeon

Croatian name: jesetra

Range in Croatia: Danube drainage - Danube, Drava and Sava Rivers

Literature: Fitzinger and Heckel (1836); Bonaparte (1846); Heckel and Kner (1858); Mojsisovics (1883); Mojsisovics (1884); Glowacki (1885); Anonymous (1886); Anonymous (1886); Seeley (1886); Dudan (1893); Kišpatić (1893); Glowacki (1896); Hirc (1897); Mojsisovics (1897); Brusina (1902); Ćurčić (1910); Vutskits (1918); Maldini (1935a); Pešić (1955); Sabioncello (1967); Vuković and Ivanović (1971); Klašterka (1979); Mikuska (1979); Sofradžija and Hadžiselimović (1981); Mikuska (1983b); Lelek (1987); Vlasenko et al. (1989); Hensel and Holčík (1997); Jirásek and Fašaić (2000); Bloesch et al. (2006a); Bloesch et al. (2006b); Mrakovčić et al. (2006); Sofradžija (2009); Schmall and Friedrich (2014b); Schmall and Friedrich (2014d)

Remarks: regionally extinct species

\section{EX2 Acipenser naccarii Bonaparte, 1836}

Names under which the taxon was mentioned: Acipenser aculeatus Lovetsky, 1834; Acipenser heckelii Brandt \& Ratzeburg, 1833; Acipenser nardoi Heckel, 1851; Acipenser nasus Heckel, 1847 


\section{English name: Adriatic sturgeon}

Croatian name: jadranska jesetra

Range in Croatia: Adriatic Basin - Neretva and Cetina Rivers

Literature: Bonaparte (1846); Heckel and Kner (1858); Perugia (1881); Seeley (1886); Kolombatović (1894); Brusina (1902); Kosić (1903); Katurić (1903); Langhoffer (1904); Maldini (1936c); Taler (1951b); Taler (1951f); Sabioncello (1967); Vuković and Ivanović (1971); Sofradžija and Hadžiselimović (1981); Bojčić et al. (1982); Tvrtković (1985); Lelek (1987); Tortonese (1989); Crivelli (1996); Jardas (1996); Jardas et al. (2008); Leiner (1998b); Jirásek and Fašaić (2000); Zerunian (2004); Bloesch et al. (2006a); Bloesch et al. (2006b); Mrakovčić et al. (2006); Kottelat and Freyhof (2007); Valić et al. (2008); Tvrtković et al. (2009); Sofradžija (2009); Glamuzina et al. (2010b); Glamuzina et al. (2013); Schmall and Friedrich (2014d); Ćaleta et al. (2015); Piria et al. (2016b)

Remarks: regionally extinct species in freshwater habitats

\section{Acipenser nudiventris Lovetsky, 1828}

Names under which the taxon was mentioned: Acipenser glaber Fitzinger, 1836; Acipenser glaber Fitzinger, 1836; Acipenser schypa Güldenstädt, 1772

English name: Ship sturgeon

Croatian name: sim

Range in Croatia: Danube drainage - Danube, Drava, Mura, Sava, Una, Kupa and Lonja Rivers

Literature: Marsigli (1726); Fitzinger and Heckel (1836); Bonaparte (1846); Heckel and Kner (1858); Sebišanović (1880); Mojsisovics (1883); Frick (1883); Mojsisovics (1884); Glowacki (1885); Anonymous (1886); Seeley (1886); Brusina (1892); Dudan (1893); Kišpatić (1893); Glowacki (1896); Brusina (1902); Langhoffer (1904); RHZZM (1908); Ćurčić (1910); Vutskits (1918); Plančić (1923b); Tadić (1932); Trojanović (1934); Maldini (1935a); Fink (1947); Anonymous (1953b); Taler (1953a); Pešić (1955); Berinkey (1966); Sabioncello (1967); Vuković and Ivanović (1971); Mikuska (1979); Sofradžija and Hadžiselimović (1981); Mikuska (1983b); Lelek (1987); Sokolova and Vasil'ev (1989b); Harka (1992); Hensel and Holčík (1997); Jirásek and Fašaić (2000); Sallai and Kontos (2005); Simonović et al. (2005); Bloesch et al. (2006a); Bloesch et al. (2006b); Guti (2006); Lenhardt et al. (2006); Mrakovčić et al. (2006); Sallai and Mrakovčić (2007); Sallai and Kontos (2008); Sofradžija (2009); Freyhof (2012); Jelić et al. (2012); Schmall and Friedrich (2014b); Schmall and Friedrich (2014d)

Remarks: over the past 20 years, only a few individuals have been reported, and likely originated from breeding programmes

\section{Acipenser ruthenus Linnaeus, 1758}

Names under which the taxon was mentioned: Acipenser gmelini Fitzinger, 1836; Acipenser pygmaeus Pallas, 1814; Acipenser ruthenus var. albinea Brusina, 1902; Acipenser ruthenus var. birostrata Brusina, 1902; Acipenser ruthenus var. obtusirostris Brusina, 1902

\section{English name: Sterlet}

Croatian name: kečiga

Range in Croatia: Danube drainage - lower rivers course

Literature: Taube (1777); Fitzinger and Heckel (1836); Heckel and Kner (1858); Brusina (1878); Jurinac (1880); Sebišanović (1880); Frick (1883); Mojsisovics (1883); Jurinac (1884); Mojsisovics (1884); Glowacki (1885); Anonymous (1886); Kesterčanek (1886); Seeley (1886); Mojsisovics (1887); Hefele (1889); Mojsisovics (1889); Brusina (1892); Franke (1892); Brusina (1893); Dudan (1893); Kišpatić (1893); Flögel (1894); Glowacki (1896); Hirc (1897); Mojsisovics (1897); Anonymous (1898b); Hawlitschek (1898); Medić (1901b); Ribić (1901); Brusina (1902); Langhoffer (1904); RHZZM (1908); Ćurčić (1910); Vutskits (1918); Plančić (1920); Plančić (1923a); Anonymous (1929b); Böhm (1929); Anonymous (1931c); Thaller (1932); Trojanović (1934); Anonymous (1935d); Jedlička (1935); Maldini (1935a); Maldini (1937); HafnerLahorski (1948b); Taler (1948b); Hafner-Lahorski (1950c); Plančić (1952d); Plančić (1952e); Pešić (1955); Jagodić (1957); Ristić (1963); Vuković (1963); Hadžić (1964c); Stepanek (1966); Sabioncello (1967); Tóth and Mikuska (1971); Vuković and Ivanović (1971); Munjko (1972); Vrebac (1972); Herga (1968); Klašterka (1979); Mikuska (1979); Sofradžija and Hadžiselimović (1981); Bojčić et al. (1982); Veljović (1982); Mikuska (1983a); Mikuska (1983b); Delić (1984); Mikuska (1984); Vrenk (1984); Tvrtković (1985); Veljović (1985); Lelek (1987); Sokolov and Vasil'ev (1989a); Homen et al. (1991); Harka (1992); KolarDimitrijević (1994); Mrakovčić et al. (1996b); Biber (1997); Hensel and Holčík (1997); Kottelat (1997); Majer (1998); Jirásek and Fašaić (2000); Mustafić (2001); Jakirčević (2005); Mustafić (2005); Sallai and Kontos (2005); Bloesch et al. (2006a); Bloesch et al. (2006b); Lenhardt et al. (2006); Mrakovčić et al. (2006); Sallai and Mrakovčić (2007); Sallai and Kontos (2008); Suić et al. (2008); Guti and Gaebele (2009); Sofradžija (2009); Ozimec et al. (2010); Labak (2011); Freyhof (2012); Jelić et al. (2012); Tomljanović et al. (2013b); Schmall and Friedrich (2014c); Schmall and Friedrich (2014d)

\section{EX3 Acipenser stellatus Pallas, 1771}

Names under which the taxon was mentioned: Acipenser stellatus danubialis Brusina, 1902; Acipenser stellatus illyricus Brusina, 1902

English name: Stellate sturgeon

Croatian name: pastruga

Range in Croatia: Danube drainage - Danube, Drava and 
Sava Rivers

Literature: Heckel and Kner (1858); Perugia (1881); Faber (1883); Frick (1883); Mojsisovics (1883); Mojsisovics (1884); Glowacki (1885); Anonymous (1886); Kolombatović (1886a); Kolombatović (1886b); Mojsisovics (1887); Kolombatović (1888); Mojsisovics (1889); Kišpatić (1893); Kolombatović (1894); Anonymous (1894g); Trois (1896); Glowacki (1896); Medić (1896); Hirc (1897); Mojsisovics (1897); Medić (1898); Horvat (1901); Medić (1901a); Brusina (1902); Katurić (1903); Kosić (1903); Brusina (1907); Kolombatović (1907); Vutskits (1918); Plančić (1923b); Tadić (1931); Tadić (1932); Maldini (1935a); Rotarides (1944); Berg (1948); Taler (1951f); Taler (1953a); Pešić (1955); Ristić (1963); Sabioncello (1967); Vuković and Ivanović (1971); Mikuska (1983a); Mikuska (1983b); Lelek (1987); Shubina et al. (1989); Hensel and Holčík (1997); Kottelat (1997); Jirásek and Fašaić (2000); Bloesch et al. (2006a); Bloesch et al. (2006b); Mrakovčić et al. (2006); Schmall and Friedrich (2014b); Schmall and Friedrich (2014d)

Remarks: regionally extinct species

\section{EX4 Acipenser sturio Linnaeus, 1758}

English name: Atlantic sturgeon

Croatian name: atlanska jesetra

Range in Croatia: Danube drainage - Danube, Drava and Sava Rivers; Adriatic Basin - Neretva River

Literature: Heckel and Kner (1858); Kolombatović (1881); Perugia (1881); Faber (1883); Kolombatović (1886a); Kolombatović (1886b); Seeley (1886); Kolombatović (1888); Hefele (1889); Kosić (1889); Brusina (1892); Kišpatić (1893); Katurić (1896d); Hawlitschek (1898); Brusina (1902); Katurić (1903); Kosić (1903); Langhoffer (1904); Kolombatović (1907); Katurić (1908); Ćurčić (1910); Thaller (1932); Anonymous (1935g); Maldini (1935a); Šoljan (1948); Taler (1951f); Taler (1953a); Basioli (1959); Sabioncello (1967); Vuković and Ivanović (1971); Klašterka (1979); Sofradžija and Hadžiselimović (1981); Bojčić et al. (1982); Tvrtković (1985); Lelek (1987); Holčík et al. (1989); Jardas (1996); Leiner (1998b); Tvrtković (1998); Jirásek and Fašaić (2000); Bloesch et al. (2006a); Bloesch et al. (2006b); Mrakovčić et al. (2006); Kottelat and Freyhof (2007); Kovačić (2008)

Remarks: regionally extinct species; reports for the Ombla River near Dubrovnik are not confirmed

HUSO Brandt \& Ratzeburg, 1833

EX5 Huso huso (Linnaeus, 1758)

Names under which the taxon was mentioned: Acipenser huso Linnaeus, 1758

English name: Beluga

Croatian name: moruna
Range in Croatia: Danube drainage - Danube, Drava, Sava and Kupa Rivers; Adriatic Basin - Neretva River

Literature: Fitzinger and Heckel (1836); Heckel and Kner (1858); Brusina (1878); Perugia (1881); Kesterčanek (1886); Seeley (1886); Šoštarić (1888); Hefele (1889); Brusina (1892); Dudan (1893); Kišpatić (1893); Kolombatović (1894); Mojsisovics (1884); Anonymous (1894g); Katurić (1896d); Hirc (1897); Hawlitschek (1898); Steuer (1899); Anonymous (1900a); Brusina (1902); Katurić (1903); Kosić (1903); Langhoffer (1904); Kolombatović (1907); Anonymous (1908a); Ćurčić (1910); Vutskits (1918); Thaller (1932); Anonymous (1935g); Maldini (1935a); Taler (1946a); Taler (1951f); Taler (1953a); Pešić (1955); Basioli (1959); Tadić (1966); Sabioncello (1967); Stevanović (1969a); Vuković and Ivanović (1971); Sofradžija and Hadžiselimović (1981); Bojčić et al. (1982); Tvrtković (1985); Lelek (1987); Holčík et al. (1989); Pirogovskiĭ et al. (1989); Jardas (1996); Bloesch et al. (2006a); Bloesch et al. (2006b); Mrakovčić et al. (2006); Kottelat and Freyhof (2007); Kovačić (2008); Schmall and Friedrich (2014a); Schmall and Friedrich (2014d)

Remarks: regionally extinct species; introduced to fish farms for aquaculture purposes (Recirculation Aquaculture Systems)

\section{FAMILY: POLYODONTIDAE Bonaparte, 1835}

POLYODON Lacepède, 1797

UI2 Polyodon spathula (Walbaum, 1792)

English name: Paddlefish

Croatian name: veslokljunka

Range in Croatia: Danube drainage - Danube River

Literature: Jelkić and Opačak (2013); Piria et al. (2016b); Piria et al. (2018)

Remarks: non-native species in Croatia; only a few individuals recorded in the Danube River, originating from breeding programmes

\subsection{ORDER: ANGUILLIFORMES}

\subsubsection{FAMILY: ANGUILLIDAE Rafinesque, 1810}

ANGUILLA Schrank, 1798

6. Anguilla anguilla (Linnaeus, 1758)

Names under which the taxon was mentioned: Anguilla eurystoma Heckel \& Kner, 1858; Anguilla fluviatilis Anslijn, 1828; Anguilla vulgaris Shaw, 1803; Muraena anguilla Linnaeus, 1758

English name: European eel

Croatian name: jegulja

Range in Croatia: Danube drainage - sparse reports; Adriatic Basin - widespread 
Literature: Fortis (1771); Fortis (1774); Taube (1777); Grisogono (1780); Rödlich (1811); Lanza (1842); Seljan (1843); Carrara (1846); Heckel and Kner (1858); Canestrini (1866); Šloser (1870); Brusina (1874); Brusina (1878); Kolombatović (1881); Faber (1883); Mojsisovics (1883); Mojsisovics (1884); Kesterčanek (1886); Kolombatović (1886a); Kolombatović (1886b); Seeley (1886); Katurić (1887); Kolombatović (1888); Kosić (1889); Mojsisovics (1889); Sebišanović (1890); Botteri (1891); Bullo (1891); Brusina (1892); Anonymous (1893d); Dudan (1893); Kišpatić (1893); Kolombatović (1893); Anonymous (1894e); Anonymous (1894f); Katurić (1896b); Katurić (1896c); Katurić (1896e); Hirc (1897); Mojsisovics (1897); Petermann (1899); Horvat (1900b); Horvat (1901); Kosić (1903); Langhoffer (1904); Largaiolli (1904); Anonymous (1906a); Anonymous (1907a); Brusina (1907); Katurić 1907); Kolombatović (1907); Anonymous (1908b); Anonymous (1908c); Brusina (1908); RHZZM (1908); Trgovčević (1908); Anonymous (1910b); Langhoffer (1910); Lorini (1910); Car (1911); Anonymous (1913b); Anonymous (1914); Fink (1920); Lorini (1921); Houška (1924); Hirc (1925); Anonymous (1928b); Anonymous (1928e); Hirtz (1928); Parenzan (1928); Wettstein (1928); Anonymous (1931e); Rössler (1931); Šoljan (1931); Thaller (1932); Gandolfi-Hornyold (1933); Tadić (1933); Hafner-Lahorski (1935b); Maldini (1935b); Gridelli (1936); Ćurčić (1938); Gandolfi-Hornyold (1938); Maldini (1938b); Dojmi (1939); Anonymous (1947d); Hafner-Lahorski (1947a); Morović (1947); Fijan (1948b); Morović (1948); Plančić (1948); Taler (1948c); Fijan (1949); Plančić (1949); Plančić (1950); Fijan (1951a); Taler (1951g); Basioli (1952); Kovačević (1952); Plančić (1952c); Plančić (1952d); Fijan (1953); Hafner-Lahorski (1953b); Plančić et al. (1953); Plančić (1953a); Plančić (1953b); Škovrlj (1953a); Taler (1953a); Taler (1954a); Anonymous (1955b); Morović (1955); Plančić (1955); Fijan (1956); Grce (1956); Basioli (1957a); Basioli (1957b); Grce (1957); Grubišić (1957); Anonymous (1958b); Basioli (1958a); Basioli (1958b); Porečnik (1958); Basioli (1959); Grce (1959); Basioli (1960); Dubac (1961); Morović (1961); Pešić (1961); Livojević (1962b); Livojević (1962a); Morović (1962); Marčić Brusina (1963); Morović (1963); Vuković (1963); Anonymous (1964); Morović (1964b); Morović (1964a); Sabioncello et al. (1964); Anonymous (1965c); Morović (1966); Stepanek (1966); Anonymous (1967); Habeković (1967); Morović (1967); Sabioncello (1967); Stevanović (1969b); Giunio (1970); Morović (1970); Basioli (1971); Vuković and Ivanović (1971); Kolaković (1972); Morović (1972); Morović (1976); Morović (1978); Klašterka (1979); Mikuska (1979); Žikić and Bertoša (1980); Bojčić et al. (1982); Veljović (1982); Mikuska (1983a); Mikuska (1983b); Ocvirk (1984); Popović et al. (1984); Treer et al. (1984); Leiner (1985); Ocvirk (1985); Tvrtković (1985); Bojčić (1987); Lelek (1987); Mišetić et al. (1989); Treer (1989); Fašaić et al. (1990); Mrakovčić and Mišetić (1990a); Povž et al. (1990); Habeković (1993); Host (1993); Leiner and Povž (1993); Peričić (1993); Curić (1994); Leiner and
Povž (1994); Leiner et al. (1995); Mrakovčić et al. (1995); Jardas (1996); Maitland and Crivelli (1996); Mrakovčić et al. (1996a); Mrakovčić et al. (1996b); Holčík and Mrakovčić (1997); Kottelat (1997); Bonacci et al. (1998); Kerovec (1998); Leiner (1998b); Pallaoro (1998); Schneider (1998); Tvrtković (1998); Mrakovčić et al. (2000a); Mrakovčić et al. (2000b); Mikavica et al. (2001); Simonović (2001); Povž (2002); Treer et al. (2002); Treer et al. (2003c); Balbo (2005); Bašić (2005); Jakirčević (2005); Matić-Skoko et al. (2005); Mustafić (2005); Pažur (2005); Treer et al. (2005); Dulčić and Glamuzina (2006); Has-Schön et al. (2006); Mrakovčić et al. (2006); Tvrtković and Veen (2006); Valić (2006); Dulčić et al. (2007); Kottelat and Freyhof (2007); Sallai and Mrakovčić (2007); Tutman et al. (2007); Valić et al. (2007); Kovačić (2008); Marguš (2008); Muhamedagić et al. (2008); Sallai and Kontos (2008); Kužir et al. (2009); Sofradžija (2009); Trrtković et al. (2009); Bukvić et al. (2010); Glamuzina (2010); Marević (2010); Valić (2010); Vardić Smrzlić (2010); Mrakovčić and Ćaleta (2011); Jelić et al. (2012); Šiljeg (2012); Glamuzina et al. (2013); Miočić-Stošić and Kovačević (2013); Duplić (2014); Piria et al. (2014); Tomljanović (2014); Babić (2016); Jelić et al. (2016); Piria et al. (2016b); Piria et al. (2016c); Piria et al. (2016d); Mustafić et al. (2017); Pofuk et al. (2017)

Remarks: in the Danube drainage is very rare based on natural migration and latest reports probably originate from breeding

\subsection{ORDER: CLUPEIFORMES}

\subsubsection{FAMILY: CLUPEIDAE CUvier, 1816}

\section{ALOSA Garsault, 1764}

\section{Alosa fallax (Lacepède, 1803)}

Names under which the taxon was mentioned: Alosa alosa (Linnaeus, 1758); Alosa fallax nilotica (Geoffroy Saint-Hilaire, 1809); Alosa finta (Cuvier, 1829); Alosa vulgaris (Valenciennes, 1847)

English name: Twaite shad

Croatian name: čepa

Range in Croatia: Adriatic Basin - Neretva River drainage and Baćinska Lakes

Literature: Kolombatović (1881); Kolombatović (1886b); Kosić (1899); Botteri (1891); Kosić (1903); Katurić 1907); Morović (1948); Šoljan (1948); Taler (1953a); Morović (1958); Vuković (1961); Vuković (1962); Vuković (1963); Sabioncello (1967); Vuković and Ivanović (1971); Šoljan (1975); Morović (1976); Bojčić et al. (1982); Lelek (1987); Quignard and Douchement (1991); Mrakovčić et al. (1995); Jardas (1996); Kovačić (1998); Mrakovčić (1998); Mrakovčić et al. (2000a); Mikavica et al. (2001); Bianco (2002); Aprahamian (2003); Mrakovčić et al. (2006); Dulčić and Glamuzina (2006); Dulčić et al. (2007); Muhamedagić et al. (2008); Sofradžija (2009); Glamuzina et al. (2010b); Lipej and Dulčić (2010); Glamuzina et al. (2013); Piria et 
al. (2016b)

Remarks: reports for the Ombla River near Dubrovnik have not been confirmed

\section{EX6 Alosa immaculata Bennett, 1835}

Names under which the taxon was mentioned: Alausa vulgaris Valenciennes, 1847; Alosa alosa (Linnaeus, 1758); Alosa caspia nordmanni Antipa, 1904; Alosa finta (Cuvier, 1829); Alosa kessleri pontica (Eichwald, 1838); Alosa pontica (Eichwald, 1838); Alosa vulgaris (Valenciennes, 1847); Caspialosa nordmanni Knipowitsch, 1923

English name: Pontic shad

Croatian name: dunavska haringa

Range in Croatia: Danube drainage - Danube, Drava and Sava Rivers

Literature: Mojsisovics (1884); Mojsisovics (1887); Mojsisovics (1897); Glowacki (1885); Sabioncello (1967); Vuković and Ivanović (1971); Mikuska (1979); Bojčić et al. (1982); Mikuska (1983b); Mrakovčić et al. (2006); Sofradžija 2009)

Remarks: regionally extinct species

\subsection{ORDER: CYPRINIFORMES}

\subsubsection{FAMILY: COBITIDAE Swainson, 1838}

COBITIS Linnaeus, 1758

8. Cobitis bilineata Canestrini, 1865

Names under which the taxon was mentioned: Cobitis taenia bilineata Canestrini, 1865; Cobitis taenia dalmatina Karaman, 1928

English name: Italian spined loach

Croatian name: dvoprugasti vijun

Range in Croatia: Danube drainage - Plitvice Lakes; Adriatic Basin - Zrmanja River drainage

Literature: Mrakovčić et al. (1995); Mrakovčić et al. (2000c); Schneider et al. (2000b); Bohlen and Ráb (2001); Freyhof and Stelbrink (2007); Kottelat and Freyhof (2007); Buj et al. (2008b); Mrakovčić et al. (2008); Mustafić et al. (2008); Perdices et al. (2008); Zrnčić et al. (2009b); Buj (2010a); Miočić-Stošić and Kovačević (2013); Buj et al. (2014); Buj et al. (2015a); Buj et al. (2015c)

Remarks: taxonomic investigation of specimens from the Plitvice Lakes, previously reported as $C$. taenia and $C$. elongatoides, proved to be $C$. bilineata

\section{Cobitis dalmatina Karaman, 1928}

Names under which the taxon was mentioned: Cobitis taenia Linnaeus, 1758; Cobitis taenia dalmatina Karaman, 1928

English name: Dalmatian spined loach

\section{Croatian name: cetinski vijun}

Range in Croatia: Adriatic Basin - Cetina River drainage

Literature: Giglioli (1880); Faber (1883); Katurić (1883); Kolombatović (1886a); Kolombatović (1888); Katurić (1896a); Langhoffer (1904); Brusina (1907); Karaman (1928); Karaman (1929); Rössler (1931); Taler (1953a); Sabioncello (1967); Marko et al. (1968); Vuković and Ivanović (1971); Bojčić et al. (1982); Lelek (1987); Povž et al. (1990); Habeković (1993); Mrakovčić et al. (1995); Kottelat (1997); Kerovec (1998); Schneider (1998); Tvrtković (1998); Nocita and Vanni (1999); Habeković and Mišetić (2000); Mrakovčić et al. (2000c); Schneider et al. (2000b); Bohlen and Ráb (2001); Odak et al. (2002); Mateš (2004); Mustafić (2005); Mrakovčić et al. (2006); Freyhof and Stelbrink (2007); Kottelat and Freyhof (2007); Buj et al. (2008b); Duplić (2008); Jelić et al. (2008); Mrakovčić et al. (2008); Mustafić et al. (2008); Perdices et al. (2008); Šanda et al. (2008); Tvrtković et al. (2009); Buj (2010a); Popović (2010); Mrakovčić and Ćaleta (2011); Freyhof (2012); Kottelat (2012); Buj et al. (2014); Buj et al. (2015a); Buj et al. (2015c); Ćaleta et al. (2015)

Remarks: reports for the Zrmanja River is result of misidentification; stenoendemic to the Cetina River and endemic to Croatia

\section{Cobitis elongata Heckel \& Kner, 1858}

Names under which the taxon was mentioned: Cobitis taenia Linnaeus, 1758; Cobitis taenia elongata Heckel \& Kner, 1858

English name: Balkan spined loach

Croatian name: veliki vijun

Range in Croatia: Danube drainage - Sava, Kupa and Una Rivers and their tributaries

Literature: Steindachner (1866b); Sebišanović (1880); Brusina (1892); Medić (1901b); RHZZM (1908); Maldini (1935c); Karaman (1952); Vuković and Ivanović (1971); Delić (1993); Habeković et al. (1997); Honsig-Erlenburg et al. (1997); Povž et al. (1998); Mrakovčić et al. (2000c); Povž and Šumer (2000); Bohlen and Ráb (2001); Mrakovčić et al. (2002); Delić et al. (2003a); Povž and Šumer (2003); Mustafić (2005); Mrakovčić et al. (2006); Ćaleta (2007); Ivelić et al. (2007); Kottelat and Freyhof (2007); Buj et al. (2008b); Kopjar et al. (2008); Kučinić (2008); Mičetić et al. (2008); Mrakovčić et al. (2008); Treer et al. (2008a); Dumbović et al. (2009); Sofradžija (2009); Bučar et al. (2010); Buj (2010a); Popović (2010); Piria et al. (2011b); Jakovlić et al. (2013); Delić et al. (2014a); Delić et al. (2014b); Ćaleta et al. (2015); Piria et al. (2016a); Piria et al. (2016b); Simonović et al. (2017b)

Remarks: endemic to the Danube drainage 


\section{Cobitis elongatoides Băcescu \& Mayer, 1969}

Names under which the taxon was mentioned: Cobitis taenia Linnaeus, 1758; Cobitis taenia danubialis Nalbant, 1993

English name: Danube spined loach

Croatian name: obični vijun

Range in Croatia: Danube drainage - middle and lower course of rivers

Literature: Heckel and Kner (1858); Jurinac (1880); Sebišanović (1880); Sebišanović (1881); Mojsisovics (1883); Jurinac (1884); Mojsisovics (1884); Glowacki (1885); Anonymous (1886); Mojsisovics (1889); Sebišanović (1890); Dudan (1893); Kišpatić (1893); Glowacki (1896); Hirc (1897); Hirc (1898); RHZZM (1908); Hirc (1902d); Ćurčić (1910); Franić (1910); Hirc (1911); Vutskits (1918); Karaman (1923b); Anonymous (1925); Pešić (1955); Sabioncello (1967); Zobundžija (1968); Vuković and Ivanović (1971); Mikuska (1979); Sofradžija and Hadžiselimović (1981); Bojčić et al. (1982); Veljović (1982); Mikuska (1983a); Mikuska (1983b); Leiner (1985); Habeković et al. (1986); Lelek (1987); Habeković et al. (1990); Povž and Sket (1990); Habeković and Popović (1991); Habeković et al. (1992); Mrakovčić (1992); Delić (1993); Mrakovčić et al. (1996b); Habeković et al. (1997); Honsig-Erlenburg et al. (1997); Leiner (1998a); Majer (1998); Povž et al. (1998); Schneider (1998); Mrakovčić et al. (2000c); Povž and Šumer (2000); Schneider et al. (2000b); Zanella et al. (2000); Mustafić (2001); Mrakovčić et al. (2002); Bakota et al. (2003); Delić et al. (2003a); Mustafić et al. (2003); Getz (2004); Jugović (2004); Mustafić (2005); Sallai and Kontos (2005); Mrakovčić et al. (2006); Ćaleta (2007); Ivelić et al. (2007); Kottelat and Freyhof (2007); Prpa et al. (2007); Sallai and Mrakovčić (2007); Buj et al. (2008b); Mrakovčić et al. (2008); Sallai and Kontos (2008); Sevcsik and Erös (2008); Treer et al. (2008a); Delić et al. (2009); Dumbović et al. (2009); Jelić et al. (2009); Kerestessy et al. (2009); Sofradžija (2009); Treer et al. (2009); Zrnčić et al. (2009b); Bučar et al. (2010); Buj (2010a); Jelić et al. (2010); Tvrtković (2010); Vardić Smrzlić (2010); Piria et al. (2011b); Jelić et al. (2012); Delić et al. (2014a); Žutinić et al. (2014); Huang et al. (2016); Piria et al. (2016a); Simonović et al. (2017b)

Remarks: Co-occurrence of $C$. elongatoides with allfemale triploid hybrid biotypes has been reported from the Sava and Drava River systems

\section{Cobitis illyrica Freyhof \& Stelbrink, 2007}

Names under which the taxon was mentioned: Cobitis narentana Karaman, 1928; Cobitis taenia Linnaeus, 1758; Cobitis taenia dalmatina Karaman, 1928; Cobitis taenia narentana Karaman, 1928

English name: Illyrian spined loach

Croatian name: ilirski vijun
Range in Croatia: Adriatic Basin - Prološko Blato Lake and Baćinska Lakes, Matica River near Vrgorac

Literature: Faber (1883); Katurić (1883); Kolombatović (1886a); Kolombatović (1886b (Cobitis taenia)); Kolombatović (1888); Katurić (1896a); Mrakovčić et al. (1996a); Holčík and Mrakovčić (1997); Leiner (1998b); Schneider (1998); Durand et al. (2000); Mrakovčić et al. (2000a); Schneider et al. (2000a); Miotti (2002); Zanella et al. (2003a); Freyhof and Stelbrink (2007); Kottelat and Freyhof (2007); Duplić (2008); Jelić et al. (2008); Mrakovčić et al. (2008); Mustafić et al. (2008); Šanda et al. (2008); Buj (2010a); Popović (2010); Freyhof (2012); Kottelat (2012); Buj et al. (2014); Buj et al. (2015a); Buj et al. (2015c); Ćaleta et al. (2015)

Remarks: endemic to the Adriatic Basin

\section{Cobitis jadovaensis Mustafic \& Mrakovcic, 2008}

Names under which the taxon was mentioned: Barbatula barbatula (Linnaeus, 1758); Cobitis taenia Linnaeus, 1758

English name: Jadova spined loach

Croatian name: jadovski vijun

Range in Croatia: Adriatic Basin - Jadova River

Literature: Buj et al. (2008b); Jelić et al. (2008); Buj et al. (2008b); Duplić (2008); Mrakovčić et al. (2008); Mustafić et al. (2008); Buj (2010a); Popović (2010); Freyhof (2012); Kottelat (2012); Buj et al. (2014); Buj et al. (2015c); Ćaleta et al. (2015); Mihinjač et al. (2015a); Jelić et al. (2016); Jelić and Krivek (2017)

Remarks: stenoendemic to the Jadova River and endemic to Croatia

\section{Cobitis narentana Karaman, 1928}

Names under which the taxon was mentioned: Cobitis taenia Linnaeus, 1758; Cobitis taenia narentana Karaman, 1928

English name: Neretva spined loach

Croatian name: neretvanski vijun

Range in Croatia: Adriatic Basin - Neretva River drainage

Literature: Faber (1883); Katurić (1883); Katurić (1896a); Katurić 1907); Karaman (1928); Karaman (1929); Taler (1953a); Vuković (1963); Sabioncello (1967); Vuković and Ivanović (1971); Bojčić et al. (1982); Kottelat (1997); Leiner (1998b); Schneider (1998); Mrakovčić et al. (2000c); Schneider et al. (2000a); Schneider et al. (2000b); Bohlen and Ráb (2001); Odak et al. (2002); Zanella et al. (2003a); Mrakovčić et al. (2006); Freyhof and Stelbrink (2007); Kottelat and Freyhof (2007); Buj et al. (2008b); Jelić et al. (2008); Mrakovčić et al. (2008); Mustafić et al. (2008); Šanda et al. (2008); Sofradžija (2009); Zanella et al. (2009b); Buj (2010a); Glamuzina et al. (2010b); Popović (2010); Gabelica et al. (2011); Freyhof (2012); Kottelat 
(2012); Glamuzina et al. (2013); Buj et al. (2014); Buj et al. (2015a); Buj et al. (2015c); Ćaleta et al. (2015)

Remarks: reports for the Cetina River is the result of misidentification; endemic to the Neretva River drainage

MISGURNUS Lacepède, 1803

15. Misgurnus fossilis (Linnaeus, 1758)

Names under which the taxon was mentioned: Cobitis fossilis Linnaeus, 1758

English name: Weatherfish

Croatian name: piškur

Range in Croatia: Danube drainage - lower course and backwaters of rivers; Adriatic Basin - Gacka River, Blata Field and Stajnička Jaruga creek in the Lika region

Literature: Taube (1777); Heckel and Kner (1858); Brusina (1878); Jurinac (1880); Sebišanović (1880); Sebišanović (1881); Mojsisovics (1883); Jurinac (1884); Glowacki (1885); Anonymous (1886); Kesterčanek (1886); Jurinac (1887); Hefele (1889); Mojsisovics (1889); Sebišanović (1890); Brusina (1892); Dudan (1893); Kišpatić (1893); Anonymous (1894f); Glowacki (1896); Hirc (1896); Hirc (1897); Hirc (1899d); Ivakić (1905); RHZZM (1908); Trgovčević (1908); Anonymous (1909a); Ćurčić (1910); Kempf (1910); Lang (1911); Vutskits (1918); Plančić (1920); Anonymous (1925); Kaitner (1928); Fink (1932a); Trgovčević (1932); Maldini (1935c); Taler (1947); Taler (1948c); Plančić et al. (1953); Taler (1953a); Pešić (1955); Sabioncello (1967); Zobundžija (1968); Vuković and Ivanović (1971); Klašterka (1979); Mikuska (1979); Sofradžija and Hadžiselimović (1981); Bojčić et al. (1982); Veljović (1982); Ocvirk (1984); Lelek (1987); Delić (1993); Crivelli (1995); Povž (1995); Delić et al. (1997); Leiner (1998a); Majer (1998); Mrakovčić et al. (2000c); Povž and Šumer (2000); Zanella et al. (2000); Mustafić (2001); Mrakovčić et al. (2002); Delić et al. (2003a); Eros et al. (2003); Jakirčević (2005); Mustafić (2005); Suić (2005); Mrakovčić et al. (2006); Tvrtković and Veen (2006); Ćaleta (2007); Knežević (2007); Kottelat and Freyhof (2007); Sallai and Mrakovčić (2007); Mrakovčić et al. (2008); Sallai and Kontos (2008); Delić et al. (2009); Sofradžija (2009); Bučar et al. (2010); Golub (2011); Zelić et al. (2011); Jelić et al. (2012); Jakovlić et al. (2013); Jelić et al. (2016); Pofuk et al. (2017)

Remarks: populations in the Lika region may have been introduced from the Danube drainage, although the presence of a native population in the Lika region cannot be ruled out without further investigation.

SABANEJEWIA Vladykov, 1929

16. Sabanejewia balcanica (Karaman, 1922)

Names under which the taxon was mentioned: Cobitis aurata De Filippi, 1863; Cobitis aurata balcanica Karaman, 1922; Cobitis balcanica Karaman, 1922; Sabanejewia aurata (De Filippi, 1863); Sabanejewia aurata balcanica Karaman, 1922; Sabanejewia bulgarica (Drensky 1928)

English name: Balkan golden loach

Croatian name: zlatni vijun

Range in Croatia: Danube drainage - middle and lower course of rivers

Literature: Karaman (1922); Karaman (1952); Sabioncello (1967); Vuković and Ivanović (1971); Lelek (1987); Delić (1993); Honsig-Erlenburg et al. (1997); Povž et al. (1998); Mrakovčić et al. (2000c); Povž and Šumer (2000); Delić et al. (2003a); Delića (2003); Delić et al. (2003b); Eros et al. (2003); Mustafić (2005); Sallai and Kontos (2005); Mrakovčić et al. (2006); Kottelat and Freyhof (2007); Sallai and Mrakovčić (2007); Buj et al. (2008a); Kučinić (2008); Mičetić et al. (2008); Mrakovčić et al. (2008); Sallai and Kontos (2008); Zanella et al. (2008b); Delić et al. (2009); Dumbović et al. (2009); Jelić et al. (2009); Zrnčić et al. (2009a); Bučar et al. (2010); Marešová et al. (2011); Jelić et al. (2012); Delić et al. (2014a); Delić et al. (2014b); Piria et al. (2016a)

Remarks: presence of S. bulgarica (Drensky, 1928) has not been confirmed for Croatia

\section{Sabanejewia larvata (De Filippi, 1859)}

English name: Italian loach

Croatian name: talijanski vijun

Range in Croatia: Danube drainage - Plitvice Lakes

Literature: personal data

Remarks: Recent investigation of morphology and phylogenetics (unpublished data) on material from the Plitvice Lakes that was previously reported as $C$. taenia or $C$. elongatoides, revealed the presence of $S$. larvata, which is the first report of this species in Croatia.

\subsubsection{FAMILY: NEMACHEILIDAE Regan, 1911}

BARBATULA Linck, 1790

18. Barbatula barbatula (Linnaeus, 1758)

Names under which the taxon was mentioned: Nemacheilus barbatulus (Linnaeus, 1758); Nemachilus barbatula

English name: Stone loach

Croatian name: brkica

Range in Croatia: Danube drainage - upper and middle course of rivers

Literature: Jurinac (1880); Jurinac (1884); Mojsisovics (1884); Kišpatić (1893); Hirc (1897); Vutskits (1918); Sabioncello (1967); Vuković and Ivanović (1971); Brusina (1878); Klašterka (1979); Bojčić et al. (1982); Veljović (1982); Delić (1984); Vrenk (1984); Delić (1989); Habeković et al. (1990); Harka (1992); Martinović et al. (1992); Delić 
(1993); Habeković (1993); Zrnčić (1995); Mrakovčić et al. (1996b); Delić et al. (1997); Honsig-Erlenburg et al. (1997); Kerovec (1998); Leiner (1998a); Majer (1998); Schneider (1998); Holčík and Delić (2000); Zanella et al. (2000); Mustafić (2001); Mrakovčić et al. (2002); Bakota et al. (2003); Bošnir et al. (2003); Delić et al. (2003a); Jugović (2004); Mateš (2004); Bošnir et al. (2005); Jakirčević (2005); Mustafić (2005); Mrakovčić et al. (2006); Bošnir et al. (2007); Ćaleta (2007); Prpa et al. (2007); Sallai and Mrakovčić (2007); Sallai and Kontos (2008); Šedivá et al. 2008; Treer et al. (2008a); Delić et al. (2009); Dumbović et al. (2009); Jelić et al. (2009); Sofradžija (2009); Treer et al. (2009); Bukvić et al. (2010); Jelić et al. (2010); Ozimec et al. (2010); Vardić Smrzlić (2010); Jelić et al. (2012); Piria et al. (2016a)

\subsubsection{FAMILY: ACHEILOGNATHIDAE Bleeker, 1863}

RHODEUS Agassiz, 1832

\section{Rhodeus amarus (Bloch, 1782)}

Names under which the taxon was mentioned: Cyprinus amarus Bloch, 1782; Rhodeus sericeus (Pallas, 1776); Rhodeus sericeus amarus (Bloch, 1782)

English name: European bitterling

Croatian name: gavčica

Range in Croatia: Danube drainage - widespread; Adriatic Basin - introduced to Zrmanja (personal data) and Neretva River drainages

Literature: Heckel and Kner (1858); Steindachner (1866b); Sebišanović (1880); Jurinac (1881); Glowacki (1885); Seeley (1886); Jurinac (1887); Sebišanović (1889a); Sebišanović (1890); Brusina (1892); Kišpatić (1893); Glowacki (1896); Hirc (1896); Hirc (1900a); Hirc (1902a); Hirc (1902c); Hirc (1902d); RHZZM (1908); Hirc (1911); Vutskits (1918); Anonymous (1925); Bergleitner (1925); Anonymous (1928h); Maldini (1936b); Rotarides (1944); Pešić (1955); Sabioncello (1967); Zobundžija (1968); Vuković and Ivanović (1971); Čanadjija (1977); Mikuska (1979); Sofradžija and Hadžiselimović (1981); Bojčić et al. (1982); Mikuska (1983a); Mikuska (1983b); Lelek (1987); Delić (1989); Habeković et al. (1990); Martinović et al. (1992); Mrakovčić (1992); Delić (1993); Crivelli (1995); Mrakovčić et al. (1995); Mrakovčić et al. (1996b); Habeković et al. (1997); Leiner (1998a); Majer (1998); Povž et al. (1998); Holčík (1999); Zanella et al. (2000); Mikavica et al. (2001); Mustafić (2001); Mrakovčić et al. (2002); Bakota et al. (2003); Jugović (2004); Mustafić (2005); Sallai and Kontos (2005); Ćaleta (2007); Kottelat and Freyhof (2007); Prpa et al. (2007); Sallai and Mrakovčić (2007); Sallai and Kontos (2008); Treer et al. (2008a); Zanella et al. (2008b); Delić et al. (2009); Dumbović et al. (2009); Jelić et al. (2009); Kerestessy et al. (2009); Sofradžija (2009); Treer et al. (2009); Zrnčić et al. (2009a); Bryja et al. (2010); Bučar et al. (2010); Jelić et al. (2010); Tvrtković (2010); Ištok (2011);
Piria et al. (2011b); Jelić et al. (2012); Delić et al. (2014a);

Žutinić et al. (2014); Simonović et al. (2017b)

Remarks: non-native in the Adriatic Basin

\subsubsection{FAMILY: CYPRINIDAE Rafinesque, 1815}

AULOPYGE Heckel, 1841

20. Aulopyge huegelii Heckel, 1843

Names under which the taxon was mentioned: Aulopyge hugeli Heckel 1841; Aulopyge huegeli Heckel, 1843; Aulopyge hügelii Heckel, 1843

English name: Dalmatian Barbel-gudgeon

Croatian name: oštrulja

Range in Croatia: Adriatic Basin - Krka, Čikola, Cetina, Ruda and Rumin Rivers

Literature: Heckel (1841a); Heckel (1841b); Heckel (1842); Heckel (1843); Bonaparte (1845); Bonaparte (1846); Carrara (1846); Heckel and Kner (1858); Brusina (1872); Brusina (1878); Giglioli (1880); Faber (1883); Katurić (1883); Kolombatović (1886a); Kolombatović (1886b); Seeley (1886); Kolombatović (1888); Brusina (1890); Botteri (1891); Kišpatić (1893); Katurić (1896a); Petermann (1899); Langhoffer (1904); Brusina (1907); Katurić 1907); Car (1911); Ćurčić 1917); Karaman (1923b); Koller (1928); Ćurčić (1939a); Taler (1949a); Plančić (1950); Taler (1951b); Taler (1951g); Anonymous (1953a); Škovrlj (1953a); Taler (1953a); Taler (1953f); Taler (1954a); Basioli (1958b); Pešić (1961); Aganović and Vuković and Prolić (1966); Kapetanović et al. (1966); Sabioncello (1967); Karaman (1971); Vuković and Ivanović (1971); Đurović 1975); Bojčić et al. (1982); Kottelat (1984); Tvrtković (1985); Bianco (1986); Guzina 1986); Howes (1987); Lelek (1987); Fašaić et al. (1990); Mrakovčić and Mišetić (1990a); Mrakovčić and Mišetić (1990b); Povž et al. (1990); Zupančič (1990); Habeković (1993); Habeković (1994b); Mrakovčić et al. (1995); Crivelli (1996); Maitland and Crivelli (1996); Freyhof (1997); Kottelat (1997); Kerovec (1998); Schneider (1998); Šorić and Bănărescu et al. (1999a); Nocita and Vanni (1999); Guzina (2000); Habeković and Mišetić (2000); Bless and Riehl (2002); Miotti (2002); Rüdiger and Rüdiger (2002); Tvrtković and Franičević (2002); Mustafić (2005); Mrakovčić et al. (2006); Tvrtković and Veen (2006); Valić (2006); Kottelat and Freyhof (2007); Prpa et al. (2007); Jelić et al. (2008); Marguš (2008); Treer et al. (2008a); Ćaleta et al. (2009b); Treer et al. (2009); Tvrtković et al. (2009); Popović (2010); Mrakovčić and Ćaleta (2011); Freyhof (2012); Glamuzina et al. (2013); Ćaleta et al. (2015); Landeka et al. (2015); Mustafić and Mrakovčić (2017)

Remarks: endemic to the Adriatic Basin; reports for the Zrmanja River not confirmed 
BARBUS Cuvier \& Cloquet, 1816

21. Barbus balcanicus Kotlík, Tsigenopoulos, Ráb \& Berrebi, 2002

Names under which the taxon was mentioned: Barbus caninus Bonaparte, 1839; Barbus meridionalis Risso, 1827; Barbus meridionalis petenyi Heckel, 1852; Barbus peloponnesius Valenciennes, 1842; Barbus petenyi Heckel, 1852

English name: Danube barbel

Croatian name: potočna mrena

Range in Croatia: Danube drainage - upper and middle course of rivers

Literature: Steindachner (1866b); Sebišanović (1880); Sebišanović (1881); Glowacki (1885); Mojsisovics (1887); Mojsisovics (1889); Sebišanović (1890); Brusina (1892); Brusina (1893); Kišpatić (1893); Glowacki (1896); Hirc (1898); Hirc (1899d); Hirc (1900a); Medić (1901b); Langhoffer (1904); RHZZM (1908); Vutskits (1918); Koller (1926); Kaitner (1929b); Maldini (1935b); Smlatić (1961); Sabioncello (1967); Karaman (1971); Vuković and Ivanović (1971); Klašterka (1979); Delić (1984); Popović and Fašaić (1986); Lelek (1987); Delić (1989); Delić (1993); Mrakovčić et al. (1996b); Habeković et al. (1997); Honsig-Erlenburg et al. (1997); Povž et al. (1998); Holčík and Delić (2000); Mustafić (2001); Kotlík et al. (2002); Delić et al. (2003a); Economidis et al. (2003); Domitrović et al. (2004); Jugović (2004); Piria et al. (2004); Jakirčević (2005); Piria et al. (2005b); Mrakovčić et al. (2006); Treer et al. (2006); Kottelat and Freyhof (2007); Piria (2007); Piria et al. (2007); Prpa et al. (2007); Treer et al. (2008a); Delić et al. (2009); Dumbović et al. (2009); Jelić et al. (2009); Marková et al. (2010); Sofradžija (2009); Treer et al. (2009); Bučar et al. (2010); Kraljić (2010); Perović and Tvrtković (2010); Tvrtković (2010); Jelić et al. (2012); Delić et al. (2014a); Žutinić et al. (2014); Piria et al. (2016a); Simonović et al. (2017b)

\section{Barbus barbus (Linnaeus, 1758)}

Names under which the taxon was mentioned: Barbus fluviatilis Fitzinger, 1832; Barbus vulgaris Fleming, 1828

English name: Barbel

Croatian name: mrena

Range in Croatia: Danube drainage - middle and lower course of rivers

Literature: Heckel and Kner (1858); Jurinac (1880); Sebišanović (1880); Jurinac (1884); Glowacki (1885); Anonymous (1886); Kesterčanek (1886); Seeley (1886); Jurinac (1887); Šoštarić (1888); Hefele (1889); Sebišanović (1890); Brusina (1892); Franke (1892); Brusina (1893); Dudan (1893); Kišpatić (1893); Glowacki (1896); Hirc (1896); Hirc (1897); Franić (1898); Hirc (1898); Steuer (1899); RHZZM (1908); Ćurčić (1910); Kempf (1910); Hirc (1911); Lang (1911); Vutskits (1918); Plančić (1923b);
Anonymous (1925); Anonymous (1930c); Anonymous (1931c); Fink (1932b); Trojanović (1934); Bek (1935); Maldini (1935b); Anonymous (1937b); Maldini (1938b); Taler (1945a); Anonymous (1946a); Bergleitner (1946); Hafner-Lahorski (1948b); Morović (1948); Basioli (1957a); Jagodić (1957); Sabioncello (1967); Vuković and Ivanović (1971); Čanadjija (1977); Klašterka (1979); Mikuska (1979); Bojčić et al. (1982); Veljović (1982); Mikuska (1983a); Mikuska (1983b); Delić (1984); Mikuska (1984); Vrenk (1984); Tvrtković (1985); Veljović (1985); Habeković et al. (1986); Lelek (1987); Habeković et al. (1990); Habeković and Popović (1991); Homen et al. (1991); Mrakovčić (1992); Pažur (1993); Zrnčić (1995); Mrakovčić et al. (1996b); Habeković et al. (1997); Honsig-Erlenburg et al. (1997); Majer (1998); Povž et al. (1998); Mustafić (2001); Mrakovčić et al. (2002); Bănărescu et al. (2003); Bošnir et al. (2003); Domitrović et al. (2004); Piria et al. (2004); Bošnir et al. (2005); Jakirčević (2005); Mustafić (2005); Pažur (2005); Piria et al. (2005b); Piria et al. (2005a); Sallai and Kontos (2005); Mrakovčić et al. (2006); Treer et al. (2006); Bošnir et al. (2007); Ćaleta (2007); Ivelić et al. (2007); Kottelat and Freyhof (2007); Piria (2007); Prpa et al. (2007); Sallai and Mrakovčić (2007); Sallai and Kontos (2008); Sevcsik and Erös (2008); Treer et al. (2008a); Dumbović et al. (2009); Kerestessy et al. (2009); Sofradžija (2009); Treer et al. (2009); Bučar et al. (2010); Ozimec et al. (2010); Vardić Smrzlić (2010); Matulić et al. (2011); Piria et al. (2011b); Jelić et al. (2012); Muhamedagić and Habibović (2013); Delić et al. (2014a); Piria et al. (2016a); Simonović et al. (2017b); Nedić et al. (2018); Zuliani et al. (2019)

\section{Barbus plebejus Bonaparte, 1839}

Names under which the taxon was mentioned: Barbus meridionalis caninus Almaça, 1969; Barbus balcanicus Kotlík, Tsigenopoulos, Ráb \& Berrebi, 2002; Barbus barbus (Linnaeus, 1758); Barbus barbus plebejus Bonaparte, 1839; Barbus caninus Bonaparte, 1839; Barbus eques Bonaparte, 1839; Barbus fluviatilis Fitzinger, 1832; Barbus meridionalis petenyi Heckel, 1852; Barbus plebeius Valenciennes, 1842

\section{English name: Italian barbel}

Croatian name: mren

Range in Croatia: Adriatic Basin - rivers on the Istrian Peninsula, Krka and Zrmanja River drainage

Literature: Grisogono (1780); Carrara (1846); Heckel and Kner (1858); Dybowski (1862); Canestrini (1866); Brusina (1874); Canestrini (1874); Faber (1883); Katurić (1883); Kolombatović (1886a); Kolombatović (1886b); Seeley (1886); Katurić (1887); Kolombatović (1888); Botteri (1891); Kišpatić (1893); Katurić (1896a); Katurić (1896c); Petermann (1899); Langhoffer (1904); Largaiolli (1904); Katurić 1907); Kolombatović (1907); Karaman (1923b); Koller (1926); Karaman (1928); Rössler (1931); Gridelli (1936); Hafner-Lahorski (1947a); Taler (1954a); Basioli 
(1958b); Anonymous (1967); Sabioncello (1967); Karaman (1971); Vuković and Ivanović (1971); Žikić and Bertoša (1980); Sofradžija and Hadžiselimović (1981); Bojčić et al. (1982); Almaca (1984b); Almaca (1984a); Leiner (1984); Leiner (1985); Lelek (1987); Fašaić et al. (1990); Mrakovčić and Mišetić (1990a); Povž et al. (1990); Povž (2002); Leiner and Povž (1993); Leiner and Povž (1994); Leiner et al. (1995); Bianco (1995b); Mrakovčić et al. (1995); Crivelli (1996); Maitland and Crivelli (1996); Kottelat (1997); Bianco (1998); Mrakovčić (1998); Schneider (1998); Nocita and Vanni (1999); Mrakovčić et al. (2000b); Bianco (2003); Zerunian (2004); Piria et al. (2005b); Mrakovčić et al. (2006); Kottelat and Freyhof (2007); Valić et al. (2007); Jelić et al. (2008); Marguš (2008); Bianco (2014a); Bianco (2014b); Ćaleta et al. (2015); Piria et al. (2016b)

Remarks: endemic to the Adriatic Basin

\section{CARASSIUS Jarocki, 1822}

\section{Carassius auratus (Linnaeus, 1758)}

Names under which the taxon was mentioned: Carassius auratus auratus (Linnaeus, 1758)

English name: Goldfish

Croatian name: zlatna ribica

Range in Croatia: Danube drainage and Adriatic Basin introduced locally

Literature: Maldini (1935c); Leiner (1984); Leiner (1985); Treer (1989); Leiner and Povž (1993); Delić (1993); Crivelli (1995); Leiner et al. (1995); Szczerbowski and Szczerbowski (2002); Mrakovčić et al. (2006); Prpa et al. (2007); Jakovlić and Gui (2011); Povž and Gregori (2014); Piria et al. (2016b); Jelić and Krivek (2017); Piria et al. (2018)

Remarks: non-native species in Croatia; it is possible that older reports of $C$. auratus are result of misidentification while new records are individuls of domesticated (colored) form

\section{Carassius carassius (Linnaeus, 1758)}

Names under which the taxon was mentioned: Carassius vulgaris Nordmann, 1840

\section{English name: Crucian carp}

Croatian name: karas

Range in Croatia: Danube drainage - floodplains in the drainage, introduced to the Krbavsko Field; Adriatic Basin - introduced to the Tribalj reservoir, Vransko Lake near Biograd and Jadova River in Lika region

Literature: Brusina (1878); Jurinac (1880); Sebišanović (1880); Jurinac (1881); Sebišanović (1881); Mojsisovics (1883); Jurinac (1884); Mojsisovics (1884); Glowacki (1885); Anonymous (1886); Kesterčanek (1886); Seeley (1886); Hefele (1889); Sebišanović (1889a); Sebišanović (1890); Brusina (1892); Brusina (1893); Dudan (1893); Kišpatić (1893); Anonymous (1896a); Glowacki (1896);
Horvat (1900c); Ivakić (1905); RHZZM (1908); Ćurčić (1910); Kempf (1910); Vutskits (1918); Plančić (1920); Plančić (1923b); Anonymous (1925); Rössler (1928); Fink (1932a); Thaller (1932); Trojanović (1934); Maldini (1935c); Maldini (1938b); Anonymous (1946a); Franić (1949); Taler (1951e); Plančić (1955); Grce (1956); Grce (1957); Morović (1962); Knežević (1963b); Sabioncello et al. (1964); Pešić (1955); Habeković (1967); Sabioncello (1967); Pažur (1968a); Pažur (1969c); Zobundžija (1968); Pažur (1969b); Vuković and Ivanović (1971); Fijan (1976b); Čanadjija (1977); Klašterka (1979); Mikuska (1979); Bojčić et al. (1982); Veljović (1982); Mikuska (1983a); Mikuska (1983b); Delić (1984); Mikuska (1984); Pompe-Gotal (1984); Vrenk (1984); Tvrtković (1985); Veljović (1985); Habeković et al. (1986); Lelek (1987); Mišetić et al. (1989); Treer (1989); Habeković et al. (1990); Habeković and Popović (1991); Mrakovčić (1992); Crivelli (1995); Mrakovčić et al. (1995); Povž (1995); Mrakovčić et al. (1996b); Majer (1998); Zanella et al. (2000); Mustafić (2001); Mrakovčić et al. (2002); Szczerbowski and Szczerbowski (2002); Bakota et al. (2003); Leiner (2003); Jakirčević (2005); Pažur (2005); Suić (2005); Mrakovčić et al. (2006); Knežević (2007); Kottelat and Freyhof (2007); Sallai and Mrakovčić (2007); Sallai and Kontos (2008); Sevcsik and Erös (2008); Treer et al. (2008a); Sofradžija (2009); Treer et al. (2009); Buj (2010c); Ozimec et al. (2010); Zelić et al. (2011); Jelić et al. (2012); Marčić et al. (2012); Marr et al. (2013); Jelić et al. (2016); Piria et al. (2016a); Mustafić et al. (2017); Pofuk et al. (2017)

Remarks: non-native in the Krbavsko Field and Adriatic Basin

26. Carassius gibelio (Bloch, 1782)

Names under which the taxon was mentioned: Carassius auratus (Linnaeus, 1758); Carassius auratus gibelio (Bloch, 1782); Carassius carassius (Linnaeus, 1758)

English name: Gibel carp

Croatian name: babuška

Range in Croatia: introduced to the Danube drainage and Adriatic Basin

Literature: Jurinac (1880); Jurinac (1880); Sebišanović (1880); Jurinac (1881); Jurinac (1884); Hirc (1897); Vutskits (1918); Maldini (1935c); Gridelli (1936); Plančić (1967); Sabioncello (1967); Vuković and Ivanović (1971); Mikuska (1979); Sofradžija and Hadžiselimović (1981); Bojčić et al. (1982); Povž (1982); Mikuska (1983a); Mikuska (1983b); Delić (1984); Leiner (1984); Delić (1985); Leiner (1985); Tvrtković (1985); Habeković et al. (1986); Lelek (1987); Turk et al. (1987); Delić (1989); Treer (1989); Habeković et al. (1990); Homen et al. (1991); Martinović et al. (1992); Mrakovčić (1992); Delić (1993); Habeković (1993); Leiner and Povž (1993); Habeković (1994b); Leiner and Povž (1994); Leiner et al. (1995); Mrakovčić et al. (1995); Povž (1995); Zrnčić (1995); Kajgana (1996); Mihaljević 
et al. (1996); Mrakovčić et al. (1996b); Habeković et al. (1997); Kerovec (1998); Leiner (1998b); Leiner (1998a); Majer (1998); Schneider (1998); Mrakovčić et al. (2000a); Zanella et al. (2000); Mustafić (2001); Mrakovčić et al. (2002); Szczerbowski (2002); Bakota et al. (2003); Bošnir et al. (2003); Delić et al. (2003a); Leiner (2003); Treer et al. (2003c); Jugović (2004); Piria et al. (2004); Bošnir et al. (2005); Jakirčević (2005); Mustafić (2005); Pažur (2005); Suić (2005); Mrakovčić et al. (2006); Bošnir et al. (2007); Dulčić et al. (2007); Ćaleta (2007); Knežević (2007); Kottelat and Freyhof (2007); Sallai and Mrakovčić (2007); Valić et al. (2007); Duplić (2008); Sallai and Kontos (2008); Treer et al. (2008a); Valić et al. (2008); Delić et al. (2009); Dumbović et al. (2009); Jelić et al. (2009); Kerestessy et al. (2009); Tvrtković et al. (2009); Piria et al. (2009); Sofradžija (2009); Treer et al. (2009); Bukvić et al. (2010); Jelić et al. (2010); Jelkić et al. (2010); Ozimec et al. (2010); Treer et al. (2011); Vardić Smrzlić (2010); Matulić et al. (2011); Mrakovčić and Ćaleta (2011); Jelić et al. (2012); Marčić et al. (2012); Tomljanović et al. (2012b); Marr et al. (2013); Rylková et al. (2013); Zrnčić et al. (2013); Delić et al. (2014a); Duplić (2014); Nedić et al. (2014a); Nedić et al. (2014b); Povž and Gregori (2014); Riđanović et al. (2014); Žutinić et al. (2014); Piria et al. (2016a); Piria et al. (2016b); Piria et al. (2016c); Mustafić et al. (2017); Simonović et al. (2017b); Nedić et al. (2018); Piria et al. (2018)

Remarks: non-native, invasive species presence of Carassius langsdorfii Temminck \& Schlegel, 1846 was not confirmed for Croatia although it was reported from Neretva river basin (Hutovo blato wetland) in Bosnia and Herzegovina (Kalous et al. 2013) very close to Croatian border

CYPRINUS Linnaeus, 1758

27. Cyprinus carpio Linnaeus, 1758

Names under which the taxon was mentioned: Cyprinus acuminatus Heckel \& Kner, 1858; Cyprinus carpio carpio Linnaeus, 1758

English name: Common carp

Croatian name: šaran

Range in Croatia: Danube drainage - middle and lower course of the rivers, lakes and reservoirs; Adriatic Basin introduced to rivers and reservoirs

Literature: Taube (1777); Grisogono (1780); Heckel and Kner (1858); Šloser (1870); Brusina (1878); Jurinac (1880); Sebišanović (1880); Borne (1881); Mojsisovics (1883); Jurinac (1884); Glowacki (1885); Anonymous (1886); Kesterčanek (1886); Seeley (1886); Jurinac (1887); Hefele (1889); Sebišanović (1890); Dudan (1891); Brusina (1892); Anonymous (1893a); Brusina (1893); Dudan (1893); Kišpatić (1893); Anonymous (1894a); Flögel (1894); Anonymous (1896a); Glowacki (1896); Hirc (1896); Anonymous (1897a); Hirc (1897); Zagorac (1897); Anonymous (1899b); Anonymous (1899d); Aue (1900);
Hirc (1900b); Largaiolli (1904); Anonymous (1905a); Ivakić (1905); Peheim (1905); Anonymous (1906d); Anonymous (1906e); Anonymous (1907a); Anonymous (1907b); RHZZM (1908); Anonymous (1909a); Anonymous (1910b); Ćurčić (1910); Lorini (1910); Rössler (1910); Anonymous (1911); Hruška (1911); Vutskits (1918); Plančić (1920); Plančić (1923a); Anonymous (1925); Kaitner (1926a); Anonymous (1927c); Anonymous (1927h); Anonymous (1928c); Rössler (1928); Anonymous (1929a); Anonymous (1930c); Anonymous (1931b); Anonymous (1931c); Fink (1932a); Fink (1932b); Krajačić (1932); Thaller (1932); Tadić (1933); Trojanović (1934); Anonymous (1935j); Hafner-Lahorski (1935a); Jedlička (1935); Maldini (1935c); Plančić (1935a); Samobor (1935); Maldini (1936a); Anonymous (1937b); Maldini (1938b); Anonymous (1946a); Anonymous (1947b); Anonymous (1947c); Taler (1947); Anonymous (1948b); Anonymous (1948c); Fijan (1948a); Fijan (1948b); Morović (1948); Taler (1948c); Taler (1948e); Anonymous (1949); Fijan (1949); Livojević (1949); Fijan (1950); Hafner-Lahorski (1950a); HafnerLahorski (1950b); Plančić (1950); Fijan (1951a); Fijan (1951b); Taler (1951e); Basioli (1952); Glavan (1952); Plančić (1952d); Fijan (1953); Hafner-Lahorski (1953a); Plančić (1953b); Škovrlj (1953a); Taler (1953a); Taler (1953d); Pešić (1955); Plančić (1955); Fijan (1956); Grce (1956); Plančić (1956a); Basioli (1957a); Grce (1957); Grubišić (1957); Jagodić (1957); Basioli (1958a); Basioli (1958b); Grce (1959); Sabioncello (1959); Basioli (1960); Vuletić (1960); Sokal (1961); Livojević (1962b); Livojević (1962a); Morović (1962); Morović (1963); Fijan (1964); Morović (1964a); Sabioncello et al. (1964); Bikić (1966); Vraneš (1966); Habeković (1967); Hadžić (1967); Morović (1967); Sabioncello (1967); Pažur (1968a); Zobundžija (1968); Anonymous (1969b); Morović (1970); Sabioncello et al. (1970); Vrebac (1970); Sabioncello (1971a); Tóth and Mikuska (1971); Vuković and Ivanović (1971); Pogrmilović (1972); Vrebac (1972); Habeković (1973); Herga (1968); Fijan (1975); Munjko (1975); Fijan (1976b); Munjko (1978); Klašterka (1979); Radaković et al. (1980); Žikić and Bertoša (1980); Sofradžija and Hadžiselimović (1981); Bojčić et al. (1982); Veljović (1982); Bauer (1983); Mikuska (1983a); Delić (1984); Leiner (1984); Mikuska (1984); Mikuska and Mamić (1984); Ocvirk (1984); Pompe-Gotal (1984); Ržaničanin et al. (1984a); Ržaničanin et al. (1985); Treer et al. (1984); Vrenk (1984); Leiner (1985); Mikuska (1985); Tvrtković (1985); Habeković et al. (1986); Delić (1987); Lelek (1987); Treer (1987); Turk et al. (1987); Delić (1989); Mišetić et al. (1989); Treer (1989); Treer et al. (1989); Habeković et al. (1990); Kažić et al. (1990); Mrakovčić and Mišetić (1990a); Homen et al. (1991); Treer et al. (1991); Mrakovčić (1992); Debeljak et al. (1993); Delić (1993); Leiner and Povž (1993); Pažur (1993); Leiner and Povž (1994); Leiner et al. (1995); Mrakovčić et al. (1995); Treer et al. (1995); Turk and Debeljak (1995); Maitland and Crivelli (1996); Mihaljević et al. (1996); Mrakovčić et al. (1996b); Habeković et al. (1997); Kerovec (1998); Leiner (1998a); Leiner (1998b); Majer (1998); Mrakovčić (1998); 
Povž et al. (1998); Schneider (1998); Treer et al. (1998); Tvrtković (1998); Habeković and Mišetić (2000); Habeković and Pažur (2000); Holčík and Delić (2000); Mrakovčić et al. (2000a); Treer (2000a); Zanella et al. (2000); Mikavica et al. (2001); Mustafić (2001); Baruš et al. (2002); Mrakovčić et al. (2002); Treer et al. (2002); Delić et al. (2003a); Treer et al. (2003c); Getz (2004); Jugović (2004); Piria et al. (2004); Balbo (2005); Jakirčević (2005); Mustafić (2005); Pažur (2005); Has-Schön et al. (2006); Mrakovčić et al. (2006); Bogut et al. (2007); Domitrović et al. (2007); Dulčić et al. (2007); Ćaleta (2007); Kottelat and Freyhof (2007); Prpa et al. (2007); Sallai and Mrakovčić (2007); Sallai and Kontos (2008); Sevcsik and Erös (2008); Suić et al. (2008); Treer et al. (2008a); Valić et al. (2008); Delić et al. (2009); Jelić et al. (2009); Tvrtković et al. (2009); Sofradžija (2009); Treer et al. (2009); Bukvić et al. (2010); Jelkić et al. (2010); Ozimec et al. (2010); Treer et al. (2011); Vardić Smrzlić (2010); Zupančič et al. (2010b); Ištok (2011); Labak (2011); Matulić et al. (2011); Mrakovčić and Ćaleta (2011); Tomljanović et al. (2011); Freyhof (2012); Jelić et al. (2012); Marčić et al. (2012); Vilizzi (2012); Jelkić and Opačak (2013); Marr et al. (2013); Tomljanović et al. (2013a); Zrnčić et al. (2013); Delić et al. (2014a); Nedić et al. (2014a); Nedić et al. (2014b); Riđanović et al. (2014); Jelić et al. (2016); Pavličević et al. (2016); Piria et al. (2016a); Piria et al. (2016b); Piria et al. (2016c); Croatian State Archives (2017); Mustafić et al. (2017); Pofuk et al. (2017); Nedić et al. (2018); Piria et al. (2018)

Remarks: non-native in the Adriatic Basin; according to phylogenetic research there are not difference between wild carp populations and individuals from fish farms. Further extensive taxonomic investigation needed.

\subsubsection{FAMILY: GOBIONIDAE Bleeker, 1863}

\section{GOBIO Cuvier, 1816}

\section{Gobio obtusirostris Valenciennes, 1842}

Names under which the taxon was mentioned: Gobio fluviatilis Cuvier, 1842; Gobius fluviatilis; Gobio gobio (Linnaeus, 1758); Gobio gobio obtusirostris; Gobio vulgaris Heckel, 1837; Gobius vulgaris

English name: Danube gudgeon

Croatian name: dunavska krkuša

Range in Croatia: Danube drainage - middle and lower course of rivers; Adriatic Basin - introduced to the Mirna (Istrian penisula), Ričica and Otuča Rivers (Lika region)

Literature: Piller and Mitterpacher (1783); Heckel and Kner (1858); Steindachner (1866b); Jurinac (1880); Sebišanović (1880); Jurinac (1881); Mojsisovics (1883); Jurinac (1884); Glowacki (1885); Kesterčanek (1886); Seeley (1886); Mojsisovics (1887); Sebišanović (1888); Mojsisovics (1889); Sebišanović (1889a); Sebišanović (1890); Brusina (1892); Kišpatić (1893); Glowacki (1896); Hirc (1896); Medić (1896); Hirc (1897); Hirc (1898); Langhoffer
(1904); RHZZM (1908); Kempf (1910); Hirc (1911); Vutskits (1918); Anonymous (1925); Hafner-Lahorski (1935a); Maldini (1936a); Knop (1947c); Sabioncello (1967); Zobundžija (1968); Vuković and Ivanović (1971); Čanadjija (1977); Klašterka (1979); Mikuska (1979); Sofradžija and Hadžiselimović (1981); Bojčić et al. (1982); Veljović (1982); Mikuska (1983b); Delić (1984); Leiner (1985); Tvrtković (1985); Veljović (1985); Habeković et al. (1986); Lelek (1987); Delić (1989); Habeković and Popović (1991); Habeković et al. (1992); Mrakovčić (1992); Delić (1993); Leiner and Povž (1993); Leiner and Povž (1994); Leiner et al. (1995); Mrakovčić et al. (1995); Mrakovčić et al. (1996b); Habeković et al. (1997); Honsig-Erlenburg et al. (1997); Leiner (1998a); Majer (1998); Povž et al. (1998); Bănărescu et al. (1999a); Holčík and Delić (2000); Mrakovčić et al. (2000b); Mustafić (2001); Mrakovčić et al. (2002); Bakota et al. (2003); Delić et al. (2003a); Jugović (2004); Mateš (2004); Jakirčević (2005); Mustafić (2005); Mustafić et al. (2005); Sallai and Kontos (2005); Mrakovčić et al. (2006); Ćaleta (2007); Ivelić et al. (2007); Kottelat and Freyhof (2007); Piria (2007); Prpa et al. (2007); Sallai and Mrakovčić (2007); Sallai and Kontos (2008); Treer et al. (2008a); Zanella et al. (2008b); Delić et al. (2009); Dumbović et al. (2009); Jelić et al. (2009); Piria et al. (2009); Sofradžija (2009); Treer et al. (2009); Zrnčić et al. (2009a); Bučar et al. (2010); Buj (2010c); Jelić et al. (2010); Perović and Tvrtković (2010); Piria et al. (2011b); Jelić et al. (2012); Delić et al. (2014a); Ćaleta et al. (2015); Jelić et al. (2016); Piria et al. (2016a); Piria et al. (2016b); Pofuk et al. (2017); Simonović et al. (2017b)

Remarks: endemic to the Danube drainage; non-native in the Adriatic Basin (Istria and Lika regions); report for the Plitvice Lakes not confirmed and are likely the result of misidentification

\section{PSEUDORASBORA Bleeker, 1860}

\section{Pseudorasbora parva (Temminck \& Schlegel, 1846)}

English name: Topmouth gudgeon

\section{Croatian name: bezribica}

Range in Croatia: Danube drainage - introduced, widespread; Adriatic Basin - introduced to the Cetina and Neretva River drainages and Vransko Lake near Biograd

Literature: Tvrtković (1985); Habeković and Popović (1991); Janković and Karapetkova (1992); Martinović et al. (1992); Delić (1993); Habeković (1993); Mihaljević et al. (1996); Mrakovčić et al. (1996b); Habeković et al. (1997); Kerovec (1998); Leiner (1998b); Majer (1998); Schneider (1998); Bănărescu (1999b); Zanella et al. (2000); Mustafić (2001); Mrakovčić et al. (2002); Jugović (2004); Piria et al. (2004); Mustafić (2005); Mrakovčić et al. (2006); Ćaleta (2007); Kottelat and Freyhof (2007); Sallai and Mrakovčić (2007); Duplić (2008); Sallai and Kontos (2008); Sevcsik and Erös (2008); Delić et al. (2009); Dumbović et al. (2009); Jelić et al. (2009); Kerestessy et al. (2009); Tvrtković et al. 
(2009); Sofradžija (2009); Zrnčić et al. (2009a); Jelić et al. (2010); Ozimec et al. (2010); Mrakovčić and Ćaleta (2011); Jelić et al. (2012); Marr et al. (2013); Delić et al. (2014a); Povž and Gregori (2014); Piria et al. (2016a); Piria et al. (2016b); Mustafić et al. (2017); Piria et al. (2017); Pofuk et al. (2017); Simonović et al. (2017b); Piria et al. (2018)

Remarks: non-native, invasive species

ROMANOGOBIO Bănărescu, 1962

30. Romanogobio benacensis (Pollini, 1816)

Names under which the taxon was mentioned: Gobio gobio (Linnaeus, 1758)

English name: Italian gudgeon

Croatian name: talijanska krkuša

Range in Croatia: Adriatic Basin - Mirna River drainage

Literature: Leiner (1985); Leiner and Povž (1993); Ćaleta et al. (2015); Pofuk et al. (2017); Jelić et al. (2018)

Remarks: endemic to Adriatic Basin

\section{Romanogobio kesslerii (Dybowski, 1862)}

Names under which the taxon was mentioned: Gobio kesslerii Dybowski, 1862

English name: Kessler's gudgeon

Croatian name: Keslerova krkuša

Range in Croatia: Danube drainage - middle and lower course of rivers

Literature: Karaman (1952); Sabioncello (1967); Vuković and Ivanović (1971); Bănărescu and Nalbant (1973); Bojčić et al. (1982); Habeković et al. (1986); Lelek (1987); HonsigErlenburg et al. (1997); Povž et al. (1998); Bănărescu (1999a); Mustafić (2001); Mustafić et al. (2005); Sallai and Kontos (2005); Mrakovčić et al. (2006); Kottelat and Freyhof (2007); Sallai and Mrakovčić (2007); Sallai and Kontos (2008); Sevcsik and Erös (2008); Dumbović et al. (2009); Kerestessy et al. (2009); Sofradžija (2009); Jelić et al. (2012); Friedrich et al. (2018)

Remarks: it is possible in Croatia is present $R$. carpathorossicus, because specimens from the Kupa river used in article Friedrich et al. (2018) was identified as this species

\section{Romanogobio uranoscopus (Agassiz, 1828)}

Names under which the taxon was mentioned: Gobio uranoscopus (Agassiz, 1828)

English name: Stone gudgeon

Croatian name: tankorepa krkuša

Range in Croatia: Danube drainage - fast flowing rivers

Literature: Bonaparte (1832-1841); Heckel and Kner (1858); Siebold (1863); Jurinac (1881); Jurinac (1884);
Glowacki (1885); Seeley (1886); Sebišanović (1888); Sebišanović (1890); Kišpatić (1893); Glowacki (1896); Vutskits (1918); Sabioncello (1967); Vuković and Ivanović (1971); Bănărescu and Nalbant (1973); Bojčić et al. (1982); Lelek (1987); Mrakovčić et al. (1996b); Povž et al. (1998); Bănărescu et al. (1999b); Mustafić et al. (2005); Sallai and Kontos (2005); Mrakovčić et al. (2006); Kottelat and Freyhof (2007); Sallai and Mrakovčić (2007); Sallai and Kontos (2008); Sevcsik and Erös (2008); Ozimec et al. (2010); Ištok (2011); Jelić et al. (2012); Ćaleta et al. (2015); Piria et al. (2016a); Piria et al. (2016b); Friedrich et al. (2018)

Remarks: some literature data are likely the result of misidentification, endemic to the Danube drainage

\section{Romanogobio vladykovi (Fang, 1943)}

Names under which the taxon was mentioned: Gobio albipinnatus Lukasch, 1933; Romanogobio albipinnatus (Lukasch, 1933)

English name: Danube whitefin gudgeon

Croatian name: bjeloperajna krkuša

Range in Croatia: Danube drainage - middle and lower course of rivers

Literature: Vuković and Ivanović (1971); Bănărescu and Nalbant (1973); Bojčić et al. (1982); Delić (1993); Naseka et al. (1999); Mrakovčić et al. (2002); Mustafić (2005); Mustafić et al. (2005); Sallai and Kontos (2005); Mrakovčić et al. (2006); Ćaleta (2007); Kottelat and Freyhof (2007); Sallai and Mrakovčić (2007); Sallai and Kontos (2008); Dumbović et al. (2009); Jelić et al. (2009); Kerestessy et al. (2009); Sofradžija (2009); Jelić et al. (2010); Jelić et al. (2012); Delić et al. (2014a); Ćaleta et al. (2015); Piria et al. (2016b); Simonović et al. (2017b)

Remarks: endemic to the Danube drainage

\subsubsection{FAMILY: LEUCISCIDAE Bonaparte, 1835}

ABRAMIS Cuvier, 1816

34. Abramis brama (Linnaeus, 1758)

Names under which the taxon was mentioned: Abramis brama danubii Pavlov, 1956

English name: Bream

Croatian name: deverika

Range in Croatia: Danube drainage - middle and lower course of rivers; Adriatic Basin - introduced to the Neretva River drainage

Literature: Heckel and Kner (1858); Brusina (1878); Jurinac (1880); Sebišanović (1880); Mojsisovics (1883); Jurinac (1884); Mojsisovics (1884); Glowacki (1885); Anonymous (1886); Kesterčanek (1886); Seeley (1886); Hefele (1889); Sebišanović (1890); Brusina (1892); Brusina (1893); Dudan (1893); Kišpatić (1893); Glowacki (1896); 
Anonymous (1897b); Hirc (1897); Mojsisovics (1897); Medić (1898); RHZZM (1908); Ćurčić (1910); Vutskits (1918); Plančić (1920); Plančić (1923b); Anonymous (1925); Anonymous (1931c); Fink (1932a); Trojanović (1934); Maldini (1935a); Anonymous (1946a); Franić (1949); Pešić (1955); Sabioncello (1967); Zobundžija (1968); Sabioncello et al. (1969); Vuković and Ivanović (1971); Ristić (1972a); Vrebac (1972); Klašterka (1979); Mikuska (1979); Bojčić et al. (1982); Veljović (1982); Bauer (1983); Mikuska (1983a); Mikuska (1983b); Delić (1984); Mikuska (1984); Mikuska and Mamić (1984); Vrenk (1984); Mikuska (1985); Tvrtković (1985); Veljović (1985); Habeković et al. (1986); Delić (1989); Habeković et al. (1990); Habeković and Popović (1991); Homen et al. (1991); Martinović et al. (1992); Mrakovčić (1992); Delić (1993); Pažur (1993); Zrnčić (1995); Mihaljević et al. (1996); Mrakovčić et al. (1996b); Leiner (1998a); Majer (1998); Treer et al. (2000a); Zanella et al. (2000); Mustafić (2001); Simonović (2001); Mrakovčić et al. (2002); Bošnir et al. (2003); Treer et al. (2003a); Jugović (2004); Bošnir et al. (2005); Jakirčević (2005); Mustafić (2005); Pažur (2005); Bošnir et al. (2007); Ćaleta (2007); Kottelat and Freyhof (2007); Piria (2007); Prpa et al. (2007); Sallai and Mrakovčić (2007); Sallai and Kontos (2008); Sevcsik and Erös (2008); Suić et al. (2008); Treer et al. (2008a); Delić et al. (2009); Dumbović et al. (2009); Jelić et al. (2009); Kerestessy et al. (2009); Sofradžija (2009); Treer et al. (2009); Jelić et al. (2010); Jelkić et al. (2010); Ozimec et al. (2010); Bartulović et al. (2011); Matulić et al. (2011); Piria et al. (2011b); Jelić et al. (2012); Glamuzina et al. (2013); Jelkić and Opačak (2013); Safner et al. (2013); Zrnčić et al. (2013); Delić et al. (2014a); Nedić et al. (2014a); Nedić et al. (2014b); Riđanović et al. (2014); Dulčić and Tutman (2015); Piria et al. (2016b); Piria et al. (2016c); Pofuk et al. (2017); Simonović et al. (2017b); Nedić et al. (2018)

Remarks: non-native in the Neretva River drainage

ALBURNOIDES Jeitteles, 1861

35. Alburnoides bipunctatus (Bloch, 1782)

Names under which the taxon was mentioned: Alburnus bipunctatus (Bloch, 1782)

English name: Schneider, Spirlin

Croatian name: dvoprugasta uklija

Range in Croatia: Danube drainage - upper and middle course of rivers

Literature: Heckel and Kner (1858); Steindachner (1866b); Sebišanović (1880); Jurinac (1881); Jurinac (1884); Glowacki (1885); Anonymous (1886); Seeley (1886); Mojsisovics (1889); Sebišanović (1889a); Sebišanović (1890); Dudan (1893); Kišpatić (1893); Glowacki (1896); Hirc (1897); Hirc (1898); Medić (1901b); Langhoffer (1904); RHZZM (1908); Lang (1911); Vutskits (1918); Maldini (1935a); Sabioncello (1967); Vuković and Ivanović (1971); Klašterka (1979); Mikuska (1979); Sofradžija and Hadžiselimović (1981); Bojčić et al. (1982); Mikuska (1983b); Tvrtković (1985); Lelek (1987); Habeković and Popović (1991); Mrakovčić (1992); Mrakovčić et al. (1996b); Habeković et al. (1997); Honsig-Erlenburg et al. (1997); Povž et al. (1998); Treer et al. (2000b); Mustafić (2001); Delić et al. (2003a); Domitrović et al. (2004); Jugović (2004); Piria et al. (2004); Mustafić (2005); Piria et al. (2005b); Sallai and Kontos (2005); Mrakovčić et al. (2006); Treer et al. (2006); Ćaleta (2007); Ivelić et al. (2007); Kottelat and Freyhof (2007); Piria (2007); Prpa et al. (2007); Sallai and Mrakovčić (2007); Sallai and Kontos (2008); Treer et al. (2008a); Zanella et al. (2008b); Delić et al. (2009); Dumbović et al. (2009); Jelić et al. (2009); Kerestessy et al. (2009); Sofradžija (2009); Treer et al. (2009); Bučar et al. (2010); Buj (2010c); Jelić et al. (2010); Perović and Tvrtković (2010); Ištok (2011); Jelić et al. (2012); Delić et al. (2014a); Stierandová et al. (2016); Simonović et al. (2017b)

Remarks: probably complex of several species, Croatian freshwaters are likely inhabited by yet undescribed species (Stierandová et al. 2016)

\section{ALBURNUS Rafinesque, 1820}

36. Alburnus alburnus (Linnaeus, 1758)

Names under which the taxon was mentioned: Abramis alburnus (Linnaeus, 1758); Alburnus alburnus alburnus (Linnaeus, 1758); Abramis lucidus (Heckel, 1843); Alburnus lucidus Heckel, 1843;

English name: Bleak

Croatian name: obična uklija

Range in Croatia: Danube drainage - middle and lower course of rivers; Adriatic Basin - introduced to the Lika River system

Literature: Heckel and Kner (1858); Brusina (1878); Jurinac (1880); Sebišanović (1880); Sebišanović (1881); Jurinac (1884); Glowacki (1885); Anonymous (1886); Jurinac (1887); Hefele (1889); Mojsisovics (1889); Sebišanović (1889a); Sebišanović (1890); Brusina (1892); Brusina (1893); Dudan (1893); Kišpatić (1893); Glowacki (1896); Hirc (1896); Medić (1901b); Langhoffer (1904); RHZZM (1908); Ćurčić (1910); Hirc (1911); Lang (1911); Vutskits (1918); Plančić (1923b); Maldini (1935a); Rotarides (1944); Sabioncello (1967); Zobundžija (1968); Vuković and Ivanović (1971); Čanadjija (1977); Klašterka (1979); Mikuska (1979); Sofradžija and Hadžiselimović (1981); Bojčić et al. (1982); Mikuska (1983a); Mikuska (1983b); Mikuska and Mamić (1984); Mikuska (1985); Tvrtković (1985); Bianco (1986); Habeković et al. (1986); Lelek (1987); Delić (1989); Habeković et al. (1990); Habeković and Popović (1991); Homen et al. (1991); Mrakovčić (1992); Delić (1993); Mrakovčić et al. (1996b); Habeković et al. (1997); Leiner (1998a); Majer (1998); Povž et al. (1998); Treer et al. (1998); Zanella et al. (2000); Jakovlić and Treer (2001); Mustafić (2001); Simonović (2001); 
Mrakovčić et al. (2002); Pizzul et al. (2002); Bakota et al. (2003); Delić et al. (2003a); Leiner (2003); Domitrović et al. (2004); Jugović (2004); Piria et al. (2004); Jakirčević (2005); Mustafić (2005); Piria et al. (2005b); Treer et al. (2006); Ćaleta (2007); Ivelić et al. (2007); Kottelat and Freyhof (2007); Piria (2007); Prpa et al. (2007); Sallai and Mrakovčić (2007); Polačik et al. (2008); Sallai and Kontos (2008); Sevcsik and Erös (2008); Treer et al. (2008a); Delić et al. (2009); Dumbović et al. (2009); Jelić et al. (2009); Kerestessy et al. (2009); Sofradžija (2009); Treer et al. (2009); Zrnčić et al. (2009a); Bučar et al. (2010); Jelić et al. (2010); Jelkić et al. (2010); Ozimec et al. (2010); Vardić Smrzlić (2010); Matulić et al. (2011); Piria et al. (2011b); Jelić et al. (2012); Jelkić and Opačak (2013); Safner et al. (2013); Zrnčić et al. (2013); Delić et al. (2014a); Nedić et al. (2014a); Nedić et al. (2014b); Riđanović et al. (2014); Žutinić et al. (2014); Jelić et al. (2016); Kolarević et al. (2016); Pofuk et al. (2017); Simonović et al. (2017b); Nedić et al. (2018); Zuliani et al. (2019)

Remarks: non-native in the Lika River system

\section{Alburnus arborella (Bonaparte, 1841)}

Names under which the taxon was mentioned: Alburnus albidus (Costa, 1838); Alburnus albidus alborella (De Filippi, 1844); Alburnus alburnus (Linnaeus, 1758); Alburnus alburnus alborella (De Filippi, 1844); Alburnus alborella (De Filippi, 1844)

English name: Alborella

Croatian name: primorska uklija

Range in Croatia: Adriatic Basin - rivers on the Istrian Peninsula, Zrmanja River drainage, introduced to Ričica River and reservoirs in the Lika region

Literature: Heckel and Kner (1858); Dybowski (1862); Brusina (1878); Faber (1883); Kolombatović (1886a); Kolombatović (1886b); Kišpatić (1893); Largaiolli (1904); Karaman (1923b); Gridelli (1936); Anonymous (1967); Sabioncello (1967); Vuković and Ivanović (1971); Sofradžija and Hadžiselimović (1981); Bojčić et al. (1982); Leiner (1984); Leiner (1985); Lelek (1987); Povž et al. (1990); Leiner and Povž (1993); Leiner and Povž (1994); Leiner et al. (1995); Mrakovčić et al. (1995); Crivelli (1996); Schneider (1998); Mrakovčić et al. (2000b); Zerunian (2004); Piria et al. (2005b); Mrakovčić et al. (2006); Kottelat and Freyhof (2007); Buj et al. (2010); Miočić-Stošić and Kovačević (2013); Bianco (2014a); Bianco (2014b); Jelić et al. (2016); Jelić and Krivek (2017)

Remarks: introduced from the Zrmanja River to the Ričica River in the Lika region due to pumping of water for the Velebit pumped-storage hydro power plant; reports for the Krka River not confirmed

38. Alburnus neretvae Buj, Šanda \& Perea, 2010

Names under which the taxon was mentioned: Alburnus albidus (Costa, 1838); Alburnus albidus alborella (De Filippi, 1844); Alburnus alborella (De Filippi, 1844); Alburnus alburnellus (Martens, 1857); Alburnus alburnus alborella (De Filippi, 1844); Alburnus arborella (Bonaparte, 1841)

English name: Neretva bleak

Croatian name: neretvanska uklija

Range in Croatia: Adriatic Basin - Neretva River drainage, Matica River near Vrgorac and Baćinska Lakes

Literature: Faber (1883); Katurić (1883); Kolombatović (1886a); Kolombatović (1886b); Seeley (1886); Kolombatović (1888); Katurić (1896a); Kolombatović (1907); Vuković (1961); Kolaković (1972); Mrakovčić et al. (1995); Leiner (1998b); Tvrtković (1998); Nocita and Vanni (1999); Mrakovčić et al. (2000a); Mikavica et al. (2001); Aprahamian (2003); Kottelat and Freyhof (2007); Sofradžija (2009); Buj et al. (2010); Glamuzina et al. (2010b); Glamuzina et al. (2013); Ćaleta et al. (2015)

Remarks: endemic to the Adriatic Basin

39. Alburnus sava Bogutskaya, Zupančič, Jelić, Diripasko \& Naseka, 2017

Names under which the taxon was mentioned: Alburnus mento (Heckel, 1837); Alburnus sarmaticus Freyhof \& Kottelat, 2007; Chalcalburnus chalcoides (Güldenstädt, 1772); Chalcalburnus chalcoides danubicus (Antipa, 1909); Chalchalburnus chalcoides mento (Agassiz, 1832)

English name: Sava bleak

Croatian name: velika pliska

Range in Croatia: Danube drainage - Kupa, Mrežnica and Sava Rivers

Literature: Anonymous (1886); Sebišanović (1890); Dudan (1893); Kišpatić (1893); Glowacki (1896); Hirc (1897); Ćurčić (1910); Vutskits (1918); Maldini (1935a); Sabioncello (1967); Vuković and Ivanović (1971); Bojčić et al. (1982); Veljović (1982); Mrakovčić et al. (1996b); Honsig-Erlenburg et al. (1997); Povž et al. (1998); Mustafić (2001); Jakirčević (2005); Mrakovčić et al. (2006); Kottelat and Freyhof (2007); Sofradžija (2009); Buj (2010b); Jelić et al. (2012); Bogutskaya et al. (2017); Simonović et al. (2017b); Vucić et al. (2017)

Remarks: endemic to the Danube drainage

BALLERUS Heckel, 1843

40. Ballerus ballerus (Linnaeus, 1758)

Names under which the taxon was mentioned: Abramis ballerus (Linnaeus, 1758)

English name: Blue bream

Croatian name: kosalj

Range in Croatia: Danube drainage - middle and lower course of the rivers 
Literature: Sebišanović (1881); Mojsisovics (1883); Glowacki (1885); Anonymous (1886); Kesterčanek (1886); Seeley (1886); Brusina (1893); Dudan (1893); Kišpatić (1893); Glowacki (1896); Hirc (1897); Medić (1898); Medić (1902); RHZZM (1908); Ćurčić (1910); Vutskits (1918); Plančić (1923b); Juzbašić (1928); Fink (1932b); Trojanović (1934); Maldini (1935a); Maldini (1937); Sabioncello (1967); Zobundžija (1968); Vuković and Ivanović (1971); Čanadjija (1977); Klašterka (1979); Mikuska (1979); Sofradžija and Hadžiselimović (1981); Bojčić et al. (1982); Veljović (1982); Mikuska (1983a); Mikuska (1983b); Delić (1984); Mikuska (1984); Mikuska and Mamić (1984); Vrenk (1984); Mikuska (1985); Veljović (1985); Lelek (1987); Homen et al. (1991); Harka (1992); Mrakovčić (1992); Mrakovčić et al. (1996b); Majer (1998); Zanella et al. (2000); Mustafić (2001); Simonović (2001); Mrakovčić et al. (2002); Bakota et al. (2003); Jugović (2004); Jakirčević (2005); Mustafić (2005); Mrakovčić et al. (2006); Ćaleta (2007); Kottelat and Freyhof (2007); Sallai and Mrakovčić (2007); Sallai and Kontos (2008); Sofradžija (2009); Treer et al. (2009); Ozimec et al. (2010); Jelić et al. (2012); Jelkić and Opačak (2013); Simonović et al. (2017b)

\section{Ballerus sapa (Pallas, 1814)}

Names under which the taxon was mentioned: Abramis sapa (Pallas, 1814)

English name: White-eye bream

Croatian name: crnooka deverika

Range in Croatia: Danube drainage - middle and lower course of rivers

Literature: Heckel and Kner (1858); Glowacki (1885); Anonymous (1886); Kesterčanek (1886); Seeley (1886); Brusina (1893); Dudan (1893); Kišpatić (1893); Glowacki (1896); Mojsisovics (1897); RHZZM (1908); Ćurčić (1910); Vutskits (1918); Plančić (1923b); Anonymous (1925); Pešić (1955); Sabioncello (1967); Vuković and Ivanović (1971); Klašterka (1979); Mikuska (1979); Bojčić et al. (1982); Veljović (1982); Mikuska (1983a); Mikuska (1983b); Mikuska and Mamić (1984); Vrenk (1984); Mikuska (1985); Veljović (1985); Lelek (1987); Homen et al. (1991); Mrakovčić et al. (1996b); Leiner (1998a); Majer (1998); Mustafić (2001); Simonović (2001); Leiner (2003); Jakirčević (2005); Mustafić (2005); Mrakovčić et al. (2006); Ćaleta (2007); Kottelat and Freyhof (2007); Prpa et al. (2007); Sallai and Mrakovčić (2007); Sallai and Kontos (2008); Treer et al. (2008a); Sofradžija (2009); Treer et al. (2009); Ozimec et al. (2010); Jelić et al. (2012); Jelkić and Opačak (2013); Piria et al. (2016a); Simonović et al. (2017b); Nedić et al. (2018)

BLICCA Heckel, 1843

42. Blicca bjoerkna (Linnaeus, 1758)

Names under which the taxon was mentioned: Abramis bjoerkna (Linnaeus, 1758); Abramis blicca (Bloch, 1782);
Blicca argyroleuca Heckel, 1843

English name: White bream

Croatian name: krupatica

Range in Croatia: Danube drainage - middle and lower course of rivers

Literature: Brusina (1878); Jurinac (1880); Sebišanović (1880); Mojsisovics (1883); Jurinac (1884); Glowacki (1885); Anonymous (1886); Jurinac (1887); Hefele (1889); Mojsisovics (1889); Sebišanović (1890); Brusina (1892); Brusina (1893); Kišpatić (1893); Glowacki (1896); Mojsisovics (1897); Medić (1898); Ćurčić (1910); Vutskits (1918); Maldini (1935b); Pešić (1955); Sabioncello (1967); Vuković and Ivanović (1971); Klašterka (1979); Mikuska (1979); Sofradžija and Hadžiselimović (1981); Bojčić et al. (1982); Veljović (1982); Mikuska (1983a); Mikuska (1983b); Delić (1984); Mikuska (1984); Mikuska and Mamić (1984); Delić (1985); Mikuska (1985); Tvrtković (1985); Veljović (1985); Habeković et al. (1986); Lelek (1987); Homen et al. (1991); Mrakovčić (1992); Delić (1993); Mrakovčić et al. (1996b); Leiner (1998a); Majer (1998); Zanella et al. (2000); Mustafić (2001); Mrakovčić et al. (2002); Bakota et al. (2003); Jugović (2004); Jakirčević (2005); Mustafić (2005); Ćaleta (2007); Kottelat and Freyhof (2007); Sallai and Mrakovčić (2007); Sallai and Kontos (2008); Sevcsik and Erös (2008); Delić et al. (2009); Dumbović et al. (2009); Jelić et al. (2009); Kerestessy et al. (2009); Sofradžija (2009); Treer et al. (2009); Jelić et al. (2010); Jelkić et al. (2010); Ozimec et al. (2010); Vardić Smrzlić (2010); Matulić et al. (2011); Jelić et al. (2012); Piria et al. (2016a); Simonović et al. (2017b); Nedić et al. (2018)

\section{CHONDROSTOMA Agassiz, 1832}

\section{Chondrostoma knerii Heckel, 1843}

Names under which the taxon was mentioned: Chondrostoma kneri Heckel, 1843; Chondrostomum kneri Heckel, 1843

English name: Dalmatian nase

Croatian name: podustva

Range in Croatia: Adriatic Basin - Neretva River drainage and Baćinska Lakes

Literature: Heckel (1843); Bonaparte (1845); Bonaparte (1846); Carrara (1846); Heckel (1852); Heckel and Kner (1858); Dybowski (1862); Canestrini (1866); Canestrini (1874); Brusina (1878); Giglioli (1880); Faber (1883); Katurić (1883); Kolombatović (1886a); Kolombatović (1886b); Seeley (1886); Kolombatović (1888); Botteri (1891); Dudan (1893); Kišpatić (1893); Hamann (1896); Katurić (1896a); Petermann (1899); Langhoffer (1901); Katurić 1907); Kolombatović (1907); Brusina (1908); Karaman (1923b); Basioli (1952); Basioli (1958a); Vuković (1961); Vuković (1963); Sabioncello (1967); Kolaković (1972); Vuković and Ivanović (1971); Vuković and Kosorić (1978); Klašterka (1979); Sofradžija and Hadžiselimović 
(1981); Bojčić et al. (1982); Povž (1983); Bianco (1986); Elvira (1987); Economidis and Bănărescu (1991); Mrakovčić et al. (1995); Crivelli (1996); Elvira (1997); Kottelat (1997); Leiner (1998b); Mrakovčić (1998); Schneider (1998); Tvrtković (1998); Nocita and Vanni (1999); Mikavica et al. (2001); Aprahamian (2003); Mrakovčić et al. (2006); Glamuzina (2007); Kottelat and Freyhof (2007); Jelić et al. (2008); Muhamedagić et al. (2008); Tutman et al. (2008b); Glamuzina et al. (2010b); Freyhof (2012); Glamuzina et al. (2013); Ćaleta et al. (2015)

Remarks: endemic to the Neretva River drainage and to Adriatic Basin

\section{Chondrostoma nasus (Linnaeus, 1758)}

Names under which the taxon was mentioned: Chondrostoma nasus nasus (Linnaeus, 1758)

English name: Common nase

\section{Croatian name: podust}

Range in Croatia: Danube drainage - middle and lower course of rivers

Literature: Heckel and Kner (1858); Canestrini (1866); Canestrini (1874); Brusina (1878); Jurinac (1880); Sebišanović (1880); Sebišanović (1881); Jurinac (1884); Mojsisovics (1884); Seeley (1886); Botteri (1891); Franke (1892); Brusina (1893); Kišpatić (1893); Hirc (1897); Hirc (1902d); RHZZM (1908); Kempf (1910); Vutskits (1918); Crnić (1923); Anonymous (1925); Bergleitner (1925); Rössler (1931); Maldini (1935c); Taler (1945a); Kosorić (1955); Jagodić (1957); Vuković (1963); Marko et al. (1968); Vuković et al. (1970); Vuković and Ivanović (1971); Vrebac (1972); Čanadjija (1977); Klašterka (1979); Bojčić et al. (1982); Bianco (1986); Economidis and Bănărescu (1991); Habeković (1993); Crivelli (1996); Elvira (1997); Honsig-Erlenburg et al. (1997); Kerovec (1998); Schneider (1998); Miotti (2002); Mrakovčić et al. (2002); Hrbek et al. (2004); Pažur (2005); Mrakovčić et al. (2006); Kottelat and Freyhof (2007); Prpa et al. (2007); Jelić et al. (2008); Ćaleta et al. (2009a); Dumbović et al. (2009); Sofradžija (2009); Treer et al. (2009); Tvrtković et al. (2009); Freyhof (2012); Zrnčić et al. (2013); Delić et al. (2014a); Piria et al. (2016b); Simonović et al. (2017b); Nedić et al. (2018)

\section{Chondrostoma phoxinus Heckel, 1843}

\section{English name: Minnow nase}

\section{Croatian name: podbila}

Range in Croatia: Adriatic Basin - Cetina River drainage and Prološko Blato Lake

Literature: Carrara (1846); Heckel (1852); Heckel and Kner (1858); Dybowski (1862); Canestrini (1866); Canestrini (1874); Brusina (1878); Giglioli (1880); Faber (1883); Kolombatović (1886a); Seeley (1886); Kolombatović (1888); Botteri (1891); Kišpatić (1893); Karaman (1923a); Karaman (1923b); Koller (1928); Rössler (1931); Taler (1951g); Taler (1953a); Taler (1953f); Vuković (1963); Marko et al. (1968); Vuković et al. (1970); Vuković and Ivanović (1971); Bojčić et al. (1982); Kottelat (1984); Bianco (1986); Economidis and Bănărescu (1991); Habeković (1993); Crivelli (1996); Elvira (1997); Kerovec (1998); Schneider (1998); Miotti (2002); Hrbek et al. (2004); Mrakovčić et al. (2006); Tvrtković and Veen (2006); Kottelat and Freyhof (2007); Jelić et al. (2008); Valić et al. (2008); Ćaleta et al. (2009a); Tvrtković et al. (2009); Sofradžija (2009); Mrakovčić and Ćaleta (2011); Freyhof (2012); Ćaleta et al. (2015); Pofuk et al. (2017)

Remarks: report for the Vrba River (Krka River drainage) not confirmed; endemic to the Adriatic Basin

DELMINICHTHYS Freyhof, Lieckfeldt, Bogutskaya, Pitra \& Ludwig, 2006

46. Delminichthys adspersus (Heckel, 1843)

Names under which the taxon was mentioned: Leuciscus adspersus (Heckel, 1843); Leucos adspersus Heckel, 1843; Paraphoxinus adspersus (Heckel, 1843); Phoxinellus adspersus (Heckel, 1843); Phoxinellus scharfetteri

English name: Spotted minnow

Croatian name: imotska gaovica

Range in Croatia: Adriatic Basin - Neretva and Vrljika River drainage, Prološko Blato and Baćinska Lakes, lakes near Imotski, Matica River near Vrgorac

Literature: Heckel (1843); Bonaparte (1845); Bonaparte (1846); Carrara (1846); Heckel and Kner (1858); Dybowski (1862); Canestrini (1866); Canestrini (1874); Brusina (1878); Giglioli (1880); Steindachner (1882a); Faber (1883); Katurić (1883); Kolombatović (1886a); Kolombatović (1886b); Seeley (1886); Kolombatović (1888); Brusina (1890); Botteri (1891); Katurić (1896a); Petermann (1899); Trgovčević (1905a); Trgovčević (1905c); Katurić 1907); Kolombatović (1907); Car (1911); Karaman (1923a); Karaman (1923b); Trgovčević (1932); Maldini (1936a); Vraneš (1966); Sabioncello (1967); Bilopavlović (1970); Berberović et al. (1971); Guzina and Vuković (1971); Miladinović et al. (1971); Vuković and Ivanović (1971); Karaman (1972); Sofradžija and Hadžiselimović (1981); Bojčić et al. (1982); Vuković (1982); Bianco (1986); Crivelli (1996); Mrakovčić et al. (1996a); Holčík and Mrakovčić (1997); Kottelat (1997); Leiner (1998b); Schneider (1998); Tvrtković (1998); Nocita and Vanni (1999); Aspacher et al. (2000); Habeković and Pažur (2000); Mrakovčić et al. (2000a); Zupančič and Bogutskaya (2000); Miotti (2002); Tvrtković and Franičević (2002); Zupančič and Bogutskaya (2002); Bogutskaya and Zupančič (2003); Franičević and Tičina (2003); Freyhof et al. (2006); Mrakovčić et al. (2006); Kottelat and Freyhof (2007); Jelić et al. (2008); Šanda et al. (2008); Zupančič (2008); Sofradžija (2009); Glamuzina et al. (2010b); Palandačić et al. (2010); Gabelica et al. (2011); Freyhof (2012); Palandačić (2012a); Palandačić (2012b); Palandačić et al. (2012a); Palandačić 
et al. (2012b); Glamuzina et al. (2013); Duplić (2014); Ćaleta et al. (2015); Ozimec (2015); Mustafić et al. (2016)

Remarks: reports for the Lika region not confirmed and are likely the result of misidentification; endemic to the Adriatic Basin

\section{Delminichthys ghetaldii (Steindachner, 1882)}

Names under which the taxon was mentioned: Paraphoxinus ghetaldii Steindachner, 1882; Paraphoxinus pstrossii Steindachner, 1882; Phoxinellus ghetaldii (Steindachner, 1882);

English name: Southern Dalmatian minnow

Croatian name: popovska gaovica

Range in Croatia: Adriatic Basin - Ombla River, springs and small creeks in the Konavosko Field

Literature: Kišpatić (1893); Kosić (1903); Trgovčević (1905a); Trgovčević (1905c); Karaman (1923a); Karaman (1923b); Trgovčević (1932); Maldini (1936b); Ćurčić (1938); Taler (1953f); Sabioncello (1967); Vuković and Ivanović (1971); Sofradžija and Berberović (1972); Bianco (1986); Mrakovčić and Mišetić (1990a); Mrakovčić et al. (1995); Crivelli (1996); Maitland and Crivelli (1996); Grmek and Balabanić (2000); Tvrtković and Franičević (2002); Zupančič and Bogutskaya (2002); Bogutskaya and Zupančič (2003); Mrakovčić et al. (2006); Tvrtković and Veen (2006); Kottelat and Freyhof (2007); Jelić et al. (2008); Perea et al. (2010); Freyhof (2012); Glamuzina et al. (2013); Ćaleta et al. (2015); Jelić and Jelić (2015); Ozimec (2015); Mustafić et al. (2016)

Remarks: reports for the Krka, Vrljika and Neretva Rivers are likely the result of misidentification; endemic to the Trebišnjica River system in Bosnia and Herzegovina and Croatia

48. Delminichthys jadovensis (Zupančič \& Bogutskaya, 2002)

Names under which the taxon was mentioned: Leucos adspersus (Heckel, 1843); Paraphoxinus adspersus (Heckel, 1843); Phoxinellus jadovensis Zupančič \& Bogutskaya, 2002

English name: Jadova minnow

Croatian name: jadovska gaovica

Range in Croatia: Adriatic Basin - Jadova River

Literature: Heckel (1843); Car (1911); Taler (1953a); Zupančič and Bogutskaya (2002); Bogutskaya and Zupančič (2003); Freyhof et al. (2006); Mrakovčić et al. (2006); Kottelat and Freyhof (2007); Duplić (2008); Jelić et al. (2008); Valić et al. (2008); Palandačić et al. (2010); Perea et al. (2010); Popović (2010); Freyhof (2012); Palandačić (2012a); Ćaleta et al. (2015); Ozimec (2015); Jelić et al. (2016); Jelić and Krivek (2017); Mustafić et al. (2016)
Remarks: early reports of species of the genera Paraphoxinus and Phoxinellus in the Lika River system cannot be identified, given the presence of $T$. croaticus and $D$. jadovensis here; stenoendemic to the Jadova River and endemic to the Adriatic Basin and Croatia

49. Delminichthys krbavensis (Zupančič \& Bogutskaya, 2002)

Names under which the taxon was mentioned: Paraphoxinus ghetaldi Steindachner, 1882; Phoxinellus krbavensis Zupančič \& Bogutskaya, 2002

English name: Krbava minnow

Croatian name: krbavska gaovica

Range in Croatia: Danube drainage - Krbavsko Field

Literature: RHZZM (1908); Trgovčević (1908); Taler (1953a); Vraneš (1966); Zupančič and Bogutskaya (2002); Bogutskaya and Zupančič (2003); Mateš (2004); Freyhof et al. (2006); Mrakovčić et al. (2006); Tvrtković and Veen (2006); Kottelat and Freyhof (2007); Duplić (2008); Jelić et al. (2008); Valić et al. (2008); Palandačić et al. (2010); Perea et al. (2010); Popović (2010); Freyhof (2012); Palandačić (2012a); Palandačić (2012b); Mihinjač et al. (2015b); Ćaleta et al. (2015); Ozimec (2015); Jelić et al. (2016); Mustafić et al. (2016)

Remarks: stenoendemic to the Krbavsko Field, endemic to the Danube drainage and Croatia

LEUCASPIUS Heckel \& Kner, 1858

50. Leucaspius delineatus (Heckel, 1843)

English name: Belica

Croatian name: bjelica

Range in Croatia: Danube drainage - lower course of rivers

Literature: Giglioli (1880); Kolombatović (1886a); Seeley (1886); Kolombatović (1888); Mojsisovics (1897); Vutskits (1918); Sabioncello (1967); Vuković and Ivanović (1971); Čanadjija (1977); Klašterka (1979); Mikuska (1979); Bojčić et al. (1982); Veljović (1982); Mikuska (1983b); Veljović (1985); Lelek (1987); Harka (1992); Mrakovčić (1992); Delić (1993); Mrakovčić et al. (1996b); Nocita and Vanni (1999 (Kerka)); Zanella et al. (2000); Mustafić (2001); Jakirčević (2005); Mrakovčić et al. (2006); Kottelat and Freyhof (2007); Sallai and Mrakovčić (2007); Sallai and Kontos (2008); Jelić et al. (2009); Sofradžija (2009); Jelić et al. (2012)

Remarks: a rare species in Croatia that has only been recorded in the Žutica Forest and llova River in the past 20 years; some literature reports are likely the result of misidentification; reports in the Krka River (Adriatic Basin) not confirmed and are also likely due to misidentification 
LEUCISCUS Cuvier, 1816

51. Leuciscus aspius (Linnaeus, 1758)

Names under which the taxon was mentioned: Aspius aspius (Linnaeus, 1758); Aspius rapax Agassiz, 1835

English name: Asp

Croatian name: bolen

Range in Croatia: Danube drainage - middle and lower course of the rivers

Literature: Heckel and Kner (1858); Jurinac (1880); Sebišanović (1880); Sebišanović (1881); Jurinac (1884); Glowacki (1885); Anonymous (1886); Hefele (1889); Sebišanović (1890); Brusina (1892); Brusina (1893); Dudan (1893); Kišpatić (1893); Glowacki (1896); Hirc (1896); Anonymous (1897b); Hirc (1897); Mojsisovics (1897); Medić (1898); Horvat (1901); Medić (1901b); Šimunčić (1902); RHZZM (1908); Ćurčić (1910); Vutskits (1918); Plančić (1923a); Plančić (1923b); Anonymous (1925); Bergleitner (1925); Anonymous (1931c); Trojanović (1934); Anonymous (1935g); Maldini (1935b); Anonymous (1946a); Knop (1947a); Pešić (1955); Jagodić (1957); Sabioncello (1967); Zobundžija (1968); Sabioncello et al. (1969); Vuković and Ivanović (1971); Klašterka (1979); Mikuska (1979); Bojčić et al. (1982); Veljović (1982); Mikuska (1983a); Mikuska (1983b); Mikuska (1984); Vrenk (1984); Trrtković (1985); Veljović (1985); Lelek (1987); Delić (1989); Homen et al. (1991); Mrakovčić (1992); Delić (1993); Pažur (1993); Mrakovčić et al. (1996b); Leiner (1998a); Majer (1998); Zanella et al. (2000); Mustafić (2001); Mrakovčić et al. (2002); Jakirčević (2005); Mustafić (2005); Pažur (2005); Sallai and Kontos (2005); Mrakovčić et al. (2006); Ćaleta (2007); Kottelat and Freyhof (2007); Sallai and Mrakovčić (2007); Mateš (2008); Sallai and Kontos (2008); Sevcsik and Erös (2008); Suić et al. (2008); Dumbović et al. (2009); Jelić et al. (2009); Kerestessy et al. (2009); Sofradžija (2009); Jelić et al. (2010); Jelkić et al. (2010); Ozimec et al. (2010); Tvrtković (2010); Ištok (2011); Matulić et al. (2011); Jelić et al. (2012); Zrnčić et al. (2013); Delić et al. (2014a); Nedić et al. (2014b); Nedić et al. (2014a); Riđanović et al. (2014); Simonović et al. (2017b); Nedić et al. (2018)

\section{Leuciscus idus (Linnaeus, 1758)}

Names under which the taxon was mentioned: Idus jeses (Linnaeus, 1758); Idus melanotus (Heckel, 1843);

English name: Ide

\section{Croatian name: jez}

Range in Croatia: Danube drainage - middle and lower course of rivers

Literature: Jurinac (1880); Sebišanović (1880); Sebišanović (1881); Mojsisovics (1883); Jurinac (1884); Mojsisovics (1884); Glowacki (1885); Anonymous (1886); Seeley (1886); Hefele (1889); Mojsisovics (1889); Sebišanović (1889b); Sebišanović (1890); Brusina (1892); Dudan
(1893); Kišpatić (1893); Glowacki (1896); Hirc (1897); Mojsisovics (1897); Medić (1898); RHZZM (1908); Ćurčić (1910); Vutskits (1918); Plančić (1923b); Trojanović (1934); Maldini (1936a); Anonymous (1946a); Franić (1949); Glavan (1952); Jagodić (1957); Sabioncello (1967); Sabioncello et al. (1969); Vuković and Ivanović (1971); Munjko (1975); Mikuska (1979); Klašterka (1979); Sofradžija and Hadžiselimović (1981); Bojčić et al. (1982); Veljović (1982); Bauer (1983); Mikuska (1983a); Mikuska (1983b); Delić (1984); Mikuska (1984); Mikuska and Mamić (1984); Vrenk (1984); Mikuska (1985); Tvrtković (1985); Veljović (1985); Lelek (1987); Habeković and Popović (1991); Homen et al. (1991); Mrakovčić (1992); Pažur (1993); Mihaljević et al. (1996); Mrakovčić et al. (1996b); Leiner (1998a); Majer (1998); Zanella et al. (2000); Mustafić (2001); Mrakovčić et al. (2002); Bakota et al. (2003); Jugović (2004); Jakirčević (2005); Mustafić (2005); Pažur (2005); Mrakovčić et al. (2006); Ćaleta (2007); Kottelat and Freyhof (2007); Sallai and Mrakovčić (2007); Sallai and Kontos (2008); Suić et al. (2008); Treer et al. (2008a); Dumbović et al. (2009); Sofradžija (2009); Treer et al. (2009); Jelić et al. (2010); Jelkić et al. (2010); Ozimec et al. (2010); Matulić et al. (2011); Jelić et al. (2012); Zrnčić et al. (2013); Delić et al. (2014a); Simonović et al. (2017b)

\section{Leuciscus leuciscus (Linnaeus, 1758)}

Names under which the taxon was mentioned: Leuciscus dobula (Linnaeus, 1758); Leuciscus vulgaris Fleming, 1828; Squalius lepusculus Heckel, 1852; Squalius leuciscus Fatio, 1882

\section{English name: Common dace}

Croatian name: klenić

Range in Croatia: Danube drainage - middle and lower course of rivers

Literature: Heckel and Kner (1858); Šloser (1870); Jurinac (1881); Jurinac (1884); Glowacki (1885); Anonymous (1886); Mojsisovics (1887); Hefele (1889); Sebišanović (1890); Brusina (1892); Brusina (1893); Dudan (1893); Kišpatić (1893); Glowacki (1896); RHZZM (1908); Hirc (1911); Vutskits (1918); Anonymous (1925); Maldini (1936a); Sabioncello (1967); Pažur (1969c); Pažur (1969b); Vuković and Ivanović (1971); Mikuska (1979); Sofradžija and Hadžiselimović (1981); Bojčić et al. (1982); Veljović (1982); Mikuska (1983b); Vrenk (1984); Veljović (1985); Habeković et al. (1986); Lelek (1987); Delić (1989); Mrakovčić (1992); Delić (1993); Mrakovčić et al. (1996b); Habeković et al. (1997); Majer (1998); Treer et al. (1998); Zanella et al. (2000); Mustafić (2001); Delić et al. (2003a); Jugović (2004); Bošnir et al. (2005); Jakirčević (2005); Mustafić (2005); Bošnir et al. (2007); Kottelat and Freyhof (2007); Prpa et al. (2007); Piria (2007); Sallai and Mrakovčić (2007); Sallai and Kontos (2008); Delić et al. (2009); Jelić et al. (2009); Kerestessy et al. (2009); Sofradžija (2009); Treer et al. (2009); Zrnčić et al. (2009a); Bučar et al. (2010); Jelić 
et al. (2010); Ozimec et al. (2010); Jelić et al. (2012); Piria et al. (2016a); Pofuk et al. (2017)

Remarks: report on the introduction to the Kruščica Reservoir not confirmed

\section{PELECUS Agassiz, 1836}

54. Pelecus cultratus (Linnaeus, 1758)

English name: Ziege

Croatian name: sabljarka

Range in Croatia: Danube drainage - lower course of rivers

Literature: Heckel and Kner (1858); Brusina (1878); Sebišanović (1880); Mojsisovics (1883); Glowacki (1885); Anonymous (1886); Kesterčanek (1886); Seeley (1886); Jurinac (1887); Hefele (1889); Brusina (1892); Brusina (1893); Dudan (1893); Kišpatić (1893); Mojsisovics (1884); Glowacki (1896); Hirc (1897); Mojsisovics (1897); Medić (1902); RHZZM (1908); Ćurčić (1910); Vutskits (1918); Maldini (1936b); Pešić (1955); Sabioncello (1967); Vuković and Ivanović (1971); Klašterka (1979); Mikuska (1979); Bojčić et al. (1982); Veljović (1982); Mikuska (1983a); Mikuska (1983b); Mikuska (1984); Veljović (1985); Lelek (1987); Homen et al. (1991); Mrakovčić et al. (1996b); Majer (1998); Mustafić (2001); Jakirčević (2005); Mustafić (2005); Mrakovčić et al. (2006); Sallai and Mrakovčić (2007); Sallai and Kontos (2008); Sofradžija (2009); Ozimec et al. (2010); Jelić et al. (2012)

\section{PHOXINELLUS Heckel, 1843}

\section{Phoxinellus alepidotus Heckel, 1843}

Names under which the taxon was mentioned: Leucos adspersus Heckel, 1843; Paraphoxinus alepidotus (Heckel, 1843)

English name: Dinaric minnow

Croatian name: dinarska pijurica

Range in Croatia: Adriatic Basin - Cetina River drainage

Literature: Carrara (1846); Heckel and Kner (1858); Dybowski (1862); Bleeker (1863); Canestrini (1866); Brusina (1872); Canestrini (1874); Brusina (1878); Steindachner (1882a); Faber (1883); Katurić (1883); Kolombatović (1886a); Kolombatović (1886b); Seeley (1886); Kolombatović (1888); Brusina (1890); Katurić (1896a); Petermann (1899); Trgovčević (1905a); Brusina (1907); Katurić 1907); Car (1911); Karaman (1923b); Koller (1928); Anonymous (1953a); Taler (1953a); Taler (1953f); Pešić (1961); Sabioncello (1967); Vuković et al. (1970); Trewavas (1971); Vuković and Ivanović (1971); Sofradžija and Berberović (1972); Vuković (1977); Sofradžija and Hadžiselimović (1981); Bojčić et al. (1982); Kottelat (1984); Howes (1985); Bianco (1986); Povž et al. (1990); Crivelli (1996); Kottelat (1997); Leiner (1998b); Tvrtković (1998); Nocita and Vanni (1999 (Neretva i Cetina)); Zupančič and Bogutskaya (2000); Zupančič and Bogutskaya (2002);
Bogutskaya and Zupančič (2003); Mrakovčić et al. (2006); Tvrtković and Veen (2006); Jelić et al. (2008); Tvrtković et al. (2009); Sofradžija (2009); Mrakovčić and Ćaleta (2011); Ćaleta et al. (2015); Landeka et al. (2015); Ozimec (2015)

Remarks: reports for the Neretva River drainage not confirmed and are likely the result of misidentification; endemic to the Adriatic Basin

56. Phoxinellus dalmaticus Zupančič \& Bogutskaya, 2000

Names under which the taxon was mentioned: Paraphoxinus adspersus (Heckel, 1843)

English name: Dalmatian minnow

Croatian name: dalmatinska pijurica

Range in Croatia: Adriatic Basin - Čikola River (Krka River drainage)

Literature: Brusina (1890); Basioli (1958b); Zupančič and Bogutskaya (2000); Miotti (2002); Zupančič and Bogutskaya (2002); Bogutskaya and Zupančič (2003); Hrbek et al. (2004); Freyhof et al. (2006); Mrakovčić et al. (2006); Tvrtković and Veen (2006); Kottelat and Freyhof (2007); Duplić (2008); Jelić et al. (2008); Palandačić et al. (2010); Perea et al. (2010); Popović (2010); Freyhof (2012); Palandačić (2012a); Ćaleta et al. (2015); Landeka et al. (2015); Ozimec (2015)

Remarks: stenoendemic to the Čikola River drainage and endemic to the Adriatic Basin and Croatia

PHOXINUS Rafinesque, 1820

57. Phoxinus lumaireul Schinz, 1840

Names under which the taxon was mentioned: Leuciscus phoxinus (Linnaeus, 1758); Phoxinellus alepidotus Heckel, 1843; 2015 Phoxinus aphya (Linnaeus, 1758); Phoxinus ketmaieri Bianco \& De Bonis, 2015; Phoxinus laevis Fitzinger, 1832; Phoxinus likai Bianco \& De Bonis, Phoxinus marsilii Heckel, 1836

English name: Italian minnow

Croatian name: pijor

Range in Croatia: Danube drainage - upper course of rivers; Adriatic Basin - Krk Island, Lika region, Zrmanja River, Krka River

Literature: Carrara (1846); Heckel and Kner (1858); Steindachner (1866a); Steindachner (1866b); Katurić (1883); Glowacki (1885); Kesterčanek (1886); Kolombatović (1886a); Kolombatović (1886b); Seeley (1886); Katurić (1887); Kolombatović (1888); Sebišanović (1890); Botteri (1891); Hirc (1891); Brusina (1892); Kišpatić (1893); Anonymous (1894f); Glowacki (1896); Hirc (1896); Katurić (1896a); Katurić (1896c); Hirc (1897); Hirc (1898); Hirc (1899c); Hirc (1900a); Talpaš (1901); Langhoffer (1904); Katurić 1907); Kolombatović (1907); RHZZM (1908); Trgovčević (1908); Hirc (1911); Vutskits 
(1918); Karaman (1923b); Bergleitner (1925); Bergleitner (1928); Bergleitner (1929); Rössler (1929a); Rössler (1929b); Rössler (1930); Rössler (1931); Rössler (1932); Gridelli (1936); Maldini (1936b); Lovrić (1938); Plančić (1938); Anonymous (1945); Bergleitner (1946); Karaman (1952); Taler (1953b); Plančić (1956b); Stepanek (1956); Bogdanović (1957); Taler (1958); Bogdanović (1959); Sabioncello (1967); Pažur (1969b); Pažur (1969c); Pažur (1970); Vuković and Ivanović (1971); Čanadjija (1977); Klašterka (1979); Sofradžija and Hadžiselimović (1981); Bojčić et al. (1982); Leiner (1985); Tvrtković (1985); Bianco (1986); Lelek (1987); Mrakovčić et al. (1989); Fašaić et al. (1990); Delić (1993); Leiner and Povž (1993); Leiner and Povž (1994); Leiner et al. (1995); Mrakovčić et al. (1995); Mrakovčić et al. (1996b); Honsig-Erlenburg et al. (1997); Leiner (1998b); Povž et al. (1998); Leiner (1999); Mrakovčić et al. (2000b); Mikavica et al. (2001); Mustafić (2001); Delić et al. (2003a); Jugović (2004); Mateš (2004); Kottelat and Freyhof (2007); Prpa et al. (2007); Sallai and Mrakovčić (2007); Jalžić et al. (2008); Muhamedagić et al. (2008); Sallai and Kontos (2008); Treer et al. (2008a); Delić et al. (2009); Dumbović et al. (2009); Kerestessy et al. (2009); Sofradžija (2009); Treer et al. (2009); Bučar et al. (2010); Buj (2010c); Jelić et al. (2010); Perović and Tvrtković (2010); Trgovčić (2010); Jelić et al. (2012); Marčić et al. (2012); Miočić-Stošić and Kovačević (2013); Bianco (2014a); Delić et al. (2014a); Bianco and De Bonis (2015); Palandačić et al. (2015); Jelić et al. (2016); Palandačić et al. (2017); Vucić et al. (2018)

Remarks: dominant species of minnow in Croatia, all reports of Phoxinus phoxinus are in fact $P$. lumaireul. Recent reports (Bianco \& De Bonis, 2015; Palandačić et al., 2015) indicate the presence of more than two species (multispecies concept) of the genus Phoxinus in Croatia, though their precise distribution and taxonomic classification are still unclear. In addition to Phoxinus lumaireul, there are reports of Phoxinus karsticus and Phoxinus sp. in Croatia.

\section{Phoxinus marsilii Heckel 1836}

Names under which the taxon was mentioned: Phoxinus phoxinus (Linnaeus 1758)

English name: Marsili's minnow

Croatian name: Marsilijev pijor

Range in Croatia: Danube drainage - streams on Mt. Papuk in the Drava River system

Literature: Palandačić et al. (2017); Vucić et al. (2018)

Remarks: this species has only recently been reported for the first time in Croatia; however, its presence and distribution range have not yet been clarified.
RUTILUS Rafinesque, 1820

59. Rutilus aula (Bonaparte, 1841)

Names under which the taxon was mentioned: Gordonus aula; Leuciscus aula (Bonaparte, 1841); Leuciscus rubella Bonaparte, 1837; Leuciscus rutilus (Linnaeus, 1758); Leucos rubella Heckel, 1843; Leucos rubellicus Bonaparte, 1845; Rutilus rubilio (Bonaparte, 1837)

English name: Adriatic roach

\section{Croatian name: masnica}

Range in Croatia: Adriatic Basin - rivers of the Istrian Peninsula and small watercourses near Zadar and Nin

Literature: Heckel (1843); Bonaparte (1845); Bonaparte (1846); Carrara (1846); Heckel and Kner (1858); Dybowski (1862); Canestrini (1874); Faber (1883); Katurić (1883); Kolombatović (1886b); Kolombatović (1888); Seeley (1886); Botteri (1891); Katurić (1896d); Langhoffer (1904); Largaiolli (1904); Katurić 1907); Car (1911); Karaman (1923b); Koller (1928); Gridelli (1936); Maldini (1936a); Taler (1953a); Treer et al. (1984); Leiner (1985); Treer (1989); Povž et al. (1990); Leiner and Povž (1993); Leiner and Povž (1994); Leiner et al. (1995); Kottelat (1997); Freyhof et al. (2006); Mrakovčić et al. (2006); Kottelat and Freyhof (2007); Jelić et al. (2008); Treer et al. (2008a); Perea et al. (2010); Ćaleta et al. (2015); Piria et al. (2016b); Piria et al. (2016c)

Remarks: reports for Vransko Lake near Biograd, Tribalj Reservoir near Novi Vinodolski not confirmed and are likely due to misidentification; endemic to the Adriatic Basin

60. Rutilus basak (Heckel, 1843)

Names under which the taxon was mentioned: Leuciscus basak Heckel \& Kner 1858; Leuciscus rubella Bonaparte, 1837; Leucos aula Heckel, 1843; Leucos rubella Heckel, 1843; Rutilus basak (Heckel, 1843); Rutilus pigus (Lacepède, 1803); Rutilus rubilio (Bonaparte, 1837)

English name: Neretva roach

Croatian name: basak

Range in Croatia: Adriatic Basin - Neretva and Vrljika River drainages, Prološko Bato and Baćinska Lakes, Matica River near Vrgorac

Literature: Heckel (1843); Bonaparte (1845); Bonaparte (1846); Heckel and Kner (1858); Dybowski (1862); Canestrini (1866); Giglioli (1880); Faber (1883); Katurić (1883); Kolombatović (1886a); Kolombatović (1886b); Kolombatović (1888); Botteri (1891); Katurić (1896a); Katurić 1907); Brusina (1908); Car (1911); Karaman (1923b); Maldini (1936a); Ćurčić (1938); Basioli (1957a); Vuković and Vuković (1968); Vuković and Ivanović (1971); Sofradžija and Hadžiselimović (1981); Bojčić et al. (1982); Bianco and Taraborelli (1985); Bianco (1986); Lelek (1987); Economidis and Bănărescu (1991); Habeković 
(1993); Mrakovčić et al. (1995); Crivelli (1996); Mrakovčić et al. (1996a); Holčík and Mrakovčić (1997); Kottelat (1997); Leiner (1998b); Tvrtković (1998); Nocita and Vanni (1999); Habeković and Pažur (2000); Mrakovčić et al. (2000a); Mikavica et al. (2001); Tutman et al. (2002); Mrakovčić et al. (2006); Kottelat and Freyhof (2007); Prpa et al. (2007); Jelić et al. (2008); Ketmaier et al. (2008); Muhamedagić et al. (2008); Šanda et al. (2008); Sofradžija (2009); Glamuzina et al. (2010b); Marić (2010); Popović (2010); Glamuzina et al. (2013); Duplić (2014); Ćaleta et al. (2015); Piria et al. (2016b); Piria et al. (2016c)

Remarks: reports for the Cetina and Ruda River not confirmed and are likely the result of misidentification; endemic to the Adriatic Basin

\section{Rutilus rutilus (Linnaeus, 1758)}

Names under which the taxon was mentioned: 1836; Leuciscus rutilus (Linnaeus, 1758); Rutilus aula (Bonaparte, 1841); Rutilus rubilio (Bonaparte, 1837); Rutilus rutilus carpathorossicus Vladykov, 1930

English name: Roach

Croatian name: bodorka

Range in Croatia: Danube drainage - middle and lower course of rivers; Adriatic Basin -introduced to Cetina and Lika River drainages, Gacka River, Butoniga and Tribalj reservoirs

Literature: Heckel and Kner (1858); Brusina (1878); Jurinac (1880); Sebišanović (1880); Jurinac (1881); Sebišanović (1881); Mojsisovics (1883); Jurinac (1884); Glowacki (1885); Anonymous (1886); Kesterčanek (1886); Seeley (1886); Jurinac (1887); Hefele (1889); Mojsisovics (1889); Sebišanović (1890); Brusina (1892); Brusina (1893); Dudan (1893); Kišpatić (1893); Glowacki (1896); Hirc (1897); Medić (1898); RHZZM (1908); Ćurčić (1910); Vutskits (1918); Plančić (1923b); Trojanović (1934); Maldini (1936a); Sabioncello (1967); Pažur (1968a); Zobundžija (1968); Vuković and Aganović (1971); Vuković and Ivanović (1971); Munjko (1975); Klašterka (1979); Mikuska (1979); Sofradžija and Hadžiselimović (1981); Bojčić et al. (1982); Veljović (1982); Mikuska (1983a); Mikuska (1983b); Delić (1984); Mikuska (1984); Mikuska and Mamić (1984); Ocvirk (1984); Delić (1985); Mikuska (1985); Tvrtković (1985); Veljović (1985); Habeković et al. (1986); Habeković et al. (1990); Habeković and Popović (1991); Homen et al. (1991); Mrakovčić (1992); Povž (1995); Mihaljević et al. (1996); Mrakovčić et al. (1996b); Habeković et al. (1997); Leiner (1998a); Majer (1998); Povž et al. (1998); Treer et al. (1998); Leiner (1999); Zanella et al. (2000); Jakovlić and Treer (2001); Mustafić (2001); Šprem et al. (2001); Mrakovčić et al. (2002); Bakota et al. (2003); Bošnir et al. (2003); Leiner (2003); Jugović (2004); Bošnir et al. (2005); Jakirčević (2005); Mustafić (2005); Suić (2005); Dulčić and Glamuzina (2006); Bošnir et al. (2007); Ćaleta (2007); Knežević (2007); Kottelat and Freyhof (2007); Piria (2007);
Prpa et al. (2007); Sallai and Mrakovčić (2007); Sallai and Kontos (2008); Sevcsik and Erös (2008); Treer et al. (2008a); Dumbović et al. (2009); Jelić et al. (2009); Kerestessy et al. (2009); Sofradžija (2009); Treer et al. (2009); Zrnčić et al. (2009a); Jelić et al. (2010); Ozimec et al. (2010); Perea et al. (2010); Vardić Smrzlić (2010); Matulić et al. (2011); Piria et al. (2011b); Jelić et al. (2012); Jelkić and Opačak (2013); Zrnčić et al. (2013); Delić et al. (2014a); Jelić et al. (2016); Piria et al. (2016b); Pofuk et al. (2017); Simonović et al. (2017b); Zuliani et al. (2019)

Remarks: non-native in the Adriatic Basin

\section{Rutilus virgo (Heckel, 1852)}

Names under which the taxon was mentioned: Leuciscus pigus virgo; Leuciscus roseus Bonaparte, 1839; Leuciscus virgo Heckel, 1852; Rutilus pigus (Lacepède, 1803); Rutilus pigus virgo (Heckel, 1852)

English name: Danube roach

Croatian name: plotica

Range in Croatia: Danube drainage - middle and lower course of rivers

Literature: Bonaparte (1832-1841); Heckel and Kner (1858); Jurinac (1880); Jurinac (1884); Glowacki (1885); Seeley (1886); Sebišanović (1890); Brusina (1892); Franke (1892); Brusina (1893); Dudan (1893); Kišpatić (1893); Glowacki (1896); Hirc (1896); Hirc (1897); Hirc (1898); Hirc (1902d); Medić (1902); Langhoffer (1904); RHZZM (1908); Ćurčić (1910); Hirc (1911); Vutskits (1918); Plančić (1923b); Anonymous (1925); Anonymous (1931c); Zaplata (1932); Maldini (1936a); Maldini (1938c); Rotarides (1944); Kosorić (1955); Smlatić (1961); Sabioncello (1967); Sabioncello et al. (1969); Vuković and Ivanović (1971); Munjko (1975); Klašterka (1979); Mikuska (1979); Bojčić et al. (1982); Bauer (1983); Mikuska (1983a); Mikuska (1983b); Vrenk (1984); Tvrtković (1985); Lelek (1987); Habeković and Popović (1991); Habeković et al. (1997); Leiner (1998a); Povž et al. (1998); Povž (1999); Mustafić (2001); Mrakovčić et al. (2002); Leiner (2003); Jakirčević (2005); Mustafić (2005); Pažur (2005); Sallai and Kontos (2005); Mrakovčić et al. (2006); Ćaleta (2007); Kottelat and Freyhof (2007); Piria (2007); Prpa et al. (2007); Sallai and Mrakovčić (2007); Mateš (2008); Sallai and Kontos (2008); Treer et al. (2008a); Delić et al. (2009); Dumbović et al. (2009); Treer et al. (2009); Sofradžija (2009); Bučar et al. (2010); Popović (2010); Jelić et al. (2012); Muhamedagić and Habibović (2013); Delić et al. (2014a); Ćaleta et al. (2015); Piria et al. (2016b); Simonović et al. (2017b); Nedić et al. (2018); Zuliani et al. (2019)

Remarks: endemic to the Danube drainage 
SCARDINIUS Bonaparte, 1840

63. Scardinius dergle Heckel \& Kner, 1858

Names under which the taxon was mentioned: Leuciscus erythrophthalmus (Linnaeus, 1758) Leuciscus heegeri Agassiz, 1835, Leuciscus hesperidicus Bonaparte, 1845; Scardinius erythrophthalmus (Linnaeus, 1758); Scardinius erythropthalmus hesperidicus Heckel 1843; Scardinius erythrophthalmus krkensis; Scardinius hegeri Bonaparte, 1845; Scardinius hesperidicus Bonaparte, 1845; Scardinius scardafa (Bonaparte, 1837)

English name: Dalmatian rudd

Croatian name: drlja

Range in Croatia: Adriatic Basin - Krka and Cetina River drainages, Vransko Lake near Biograd, Vransko Lake on Cres Island

Literature: Bonaparte (1832-1841); Heckel (1843); Bonaparte (1845); Bonaparte (1846); Carrara (1846); Heckel and Kner (1858); Dybowski (1862); Canestrini (1866); Canestrini (1874); Giglioli (1880); Faber (1883); Katurić (1883); Kolombatović (1886a); Kolombatović (1886b); Seeley (1886); Kolombatović (1888); Botteri (1891); Kišpatić (1893); Katurić (1896a); Brusina (1907); Katurić 1907); Kolombatović (1907); Karaman (1923b); Hafner-Lahorski (1935b); Plančić (1950); Taler (1953a); Taler (1954a); Basioli (1958b); Pešić (1961); Vuković and Ivanović (1971); Ivanović (1972); Kosorić (1978); Klašterka (1979); Bojčić et al. (1982); Leiner (1985); Fašaić et al. (1990); Mrakovčić and Mišetić (1990a); Martinović (1995); Mrakovčić et al. (1995); Maitland and Crivelli (1996); Mrakovčić et al. (1996a); Kottelat (1997); Nocita and Vanni (1999); Topić Popović et al. (2000); Miotti (2002); Tutman et al. (2002); Mrakovčić et al. (2006); Valić (2006); Kottelat and Freyhof (2007); Valić et al. (2007); Jelić et al. (2008); Marguš (2008); Sofradžija (2009); Perea et al. (2010); Popović (2010); Valić (2010); Vardić Smrzlić (2010); Valić et al. (2013); Ćaleta et al. (2015); Landeka et al. (2015); Babić (2016); Mustafić et al. (2017)

Remarks: endemic to the Adriatic Basin, reports for the waters of the Istrian peninsula and Zrmanja River requires revision due to the lack of data in the recent period

\section{Scardinius erythrophthalmus (Linnaeus, 1758)}

Names under which the taxon was mentioned: Leuciscus erythrophthalmus (Linnaeus, 1758); Scardinius erythrophthalmus hisperidicus; Scardinius erythrophthalmus scardapha

English name: Rudd

Croatian name: crvenperka

Range in Croatia: Danube drainage - middle and lower course of rivers, introduced to the Plitvice lakes; Adriatic Basin - introduced to the Tribalj reservoir and Lika River system
Literature: Heckel and Kner (1858); Brusina (1878); Jurinac (1880); Sebišanović (1880); Jurinac (1881); Sebišanović (1881); Faber (1883); Mojsisovics (1883); Jurinac (1884); Glowacki (1885); Anonymous (1886); Sebišanović (1889a); Sebišanović (1890); Brusina (1892); Brusina (1893); Dudan (1893); Kišpatić (1893); Glowacki (1896); Hirc (1896); Hirc (1897); Medić (1898); Brusina (1907); RHZZM (1908); Ćurčić (1910); Vutskits (1918); Karaman (1923b); Plančić (1923b); Anonymous (1925); Fink (1932a); Trojanović (1934); Anonymous (1946a); Franić (1949); Taler (1953a); Basioli (1957b); Morović (1962); Vuković and Ivanišević (1962); Sabioncello et al. (1964); Habeković (1967); Sabioncello (1967); Zobundžija (1968); Sabioncello et al. (1969); Vrebac (1970); Vuković and Ivanović (1971); Ivanović (1972); Vrebac (1972); Čanadjija (1977); Klašterka (1979); Mikuska (1979); Sofradžija and Hadžiselimović (1981); Bojčić et al. (1982); Veljović (1982); Mikuska (1983a); Mikuska (1983b); Delić (1984); Mikuska (1984); Mikuska and Mamić (1984); Pompe-Gotal (1984); Vrenk (1984); Mikuska (1985); Ocvirk (1985); Tvrtković (1985); Veljović (1985); Habeković et al. (1986); Lelek (1987); Treer (1989); Habeković et al. (1990); Mrakovčić (1992); Delić (1993); Pažur (1993); Martinović (1995); Mrakovčić et al. (1995); Zrnčić (1995); Mihaljević et al. (1996); Mrakovčić et al. (1996b); Leiner (1998a); Majer (1998); Zanella et al. (2000); Jakovlić and Treer (2001); Mustafić (2001); Mrakovčić et al. (2002); Bakota et al. (2003); Jugović (2004); Jakirčević (2005); Mustafić (2005); Suić (2005); Šprem et al. (2005c); Mrakovčić et al. (2006); Ćaleta (2007); Knežević (2007); Kottelat and Freyhof (2007); Sallai and Mrakovčić (2007); Sallai and Kontos (2008); Sevcsik and Erös (2008); Treer et al. (2008a); Delić et al. (2009); Kerestessy et al. (2009); Sofradžija (2009); Treer et al. (2009); Jelić et al. (2010); Ozimec et al. (2010); Šprem et al. (2010); Treer et al. (2011); Matulić et al. (2011); Piria et al. (2011a); Jelić et al. (2012); Valić et al. (2013); Nedić et al. (2014a); Nedić et al. (2014b); Riđanović et al. (2014); Jelić et al. (2016); Piria et al. (2016b); Piria et al. (2016c); Pofuk et al. (2017)

Remarks: non-native in the Plitivice Lakes and Adriatic Basin

\section{Scardinius plotizza Heckel \& Kner, 1858}

Names under which the taxon was mentioned: Leuciscus plotizza; Leuciscus erythrophthalmus (Linnaeus, 1758) scardafa (Bonaparte, 1837); Scardinius erythrhophthalmus (Linnaeus, 1758); Scardinius erythrophthalmus scardapha (Bonaparte, 1837); Scardinius platizza Heckel, 1845; Scardinius scardafa (Bonaparte, 1837)

English name: Neretva rudd

Croatian name: peškelj

Range in Croatia: Adriatic Basin - Neretva River drainage, Baćinska Lakes and Matica River near Vrgorac

Literature: Heckel (1843); Bonaparte (1845); Bonaparte 
(1846); Carrara (1846); Heckel and Kner (1858); Dybowski (1862); Giglioli (1880); Faber (1883); Katurić (1883); Kolombatović (1886a); Kolombatović (1886b); Seeley (1886); Katurić (1887); Kolombatović (1888); Botteri (1891); Kišpatić (1893); Katurić (1896a); Katurić (1896c); Brusina (1907); Katurić 1907); Kolombatović (1907); Brusina (1908); Car (1911); Karaman (1923b); Koller (1928); Maldini (1936b); Basioli (1952); Taler (1953a); Basioli (1958a); Vuković and Ivanišević (1962); Sabioncello (1967); Vuković and Ivanović (1971); Ivanović (1972); Kolaković (1972); Morović (1976); Bojčić et al. (1982); Martinović (1995); Mrakovčić et al. (1995); Crivelli (1996); Kottelat (1997); Leiner (1998b); Tvrtković (1998); Nocita and Vanni (1999); Mrakovčić et al. (2000a); Mikavica et al. (2001); Ketmaier et al. (2004); Freyhof et al. (2005a); Mrakovčić et al. (2006); Kottelat and Freyhof (2007); Jelić et al. (2008); Bukvić et al. (2010); Glamuzina et al. (2010b); Glamuzina et al. (2013); Ćaleta et al. (2015); Tutman et al. (2016)

Remarks: endemic to the Neretva River drainage and Adriatic Basin

\section{SQUALIUS Bonaparte, 1841}

66. Squalius cephalus (Linnaeus, 1758)

Names under which the taxon was mentioned: Leuciscus cephalus (Linnaeus, 1758); Squalius dobula; Squalius albus (Bonaparte, 1838)

English name: Chub

Croatian name: obični klen

Range in Croatia: Danube drainage - widespread; Adriatic Basin - introduced to Lika River system and Tribalj reservoir

Literature: Heckel and Kner (1858); Dybowski (1862); Steindachner (1866a); Steindachner (1866b); Brusina (1878); Jurinac (1880); Sebišanović (1880); Sebišanović (1881); Mojsisovics (1883); Jurinac (1884); Mojsisovics (1884); Glowacki (1885); Kesterčanek (1886); Anonymous (1886); Seeley (1886); Jurinac (1887); Hefele (1889); Sebišanović (1889b); Sebišanović (1889a); Sebišanović (1890); Brusina (1892); Franke (1892); Anonymous (1893a); Brusina (1893); Dudan (1893); Kišpatić (1893); Anonymous (1894a); Glowacki (1896); Hirc (1896); Hirc (1897); Hirc (1898); Hirc (1900a); Hirc (1902b); Langhoffer (1904); Brusina (1907); RHZZM (1908); Ćurčić (1910); Franić (1910); Kempf (1910); Hirc (1911); Lang (1911); Vutskits (1918); Plančić (1923a); Plančić (1923b); Anonymous (1925); Bergleitner (1925); Bergleitner (1928); Kaitner (1928); Kaitner (1929b); Anonymous (1930c); Rössler (1930); Anonymous (1931c); Krajačić (1932); Hafner-Lahorski (1935a); Popović (1935); Taler (1935b); Maldini (1936b); Lovrić (1938); Taler (1945a); Anonymous (1946a); Bergleitner (1946); Knop (1947b); Taler (1947); Taler (1951e); Kosorić (1955); Plančić (1956b); Jagodić (1957); Vuletić (1960); Pešić (1961); Knežević (1963b); Vinterhalter (1963); Vraneš (1966); Sabioncello
(1967); Anonymus (1968); Pažur (1968a); Pažur (1969c); Pažur (1969b); Sabioncello et al. (1969); Pažur (1970); Vuković and Ivanović (1971); Munjko (1975); Čanadjija (1977); Klašterka (1979); Mikuska (1979); Sofradžija and Hadžiselimović (1981); Veljović (1982); Mikuska (1983a); Mikuska (1983b); Delić (1984); Ocvirk (1984); Vrenk (1984); Delić (1985); Veljović (1985); Tvrtković (1985); Habeković et al. (1986); Delić (1989); Mišetić et al. (1989); Mrakovčić et al. (1989); Habeković et al. (1990); Habeković and Popović (1991); Mrakovčić (1992); Delić (1993); Habeković et al. (1993a); Pažur (1993); Mrakovčić et al. (1996b); Habeković et al. (1997); Honsig-Erlenburg et al. (1997); Treer et al. (1997); Leiner (1998a); Majer (1998); Povž et al. (1998); Treer et al. (1998); Kolak et al. (1999); Leiner (1999); Nocita and Vanni (1999); Treer et al. (1999); Holčík and Delić (2000); Zanella et al. (2000); Jakovlić and Treer (2001); Mustafić (2001); Mrakovčić et al. (2002); Bošnir et al. (2003); Delić et al. (2003a); Leiner (2003); Domitrović et al. (2004); Jugović (2004); Mateš (2004); Piria et al. (2004); Jakirčević (2005); Mustafić (2005); Pažur (2005); Piria et al. (2005a); Piria et al. (2005b); Dulčić and Glamuzina (2006); Treer et al. (2006); Ćaleta (2007); Dragun et al. (2007); Ivelić et al. (2007); Kurtović et al. (2007); Kottelat and Freyhof (2007); Piria (2007); Prpa et al. (2007); Sallai and Mrakovčić (2007); Mateš (2008); Sallai and Kontos (2008); Sevcsik and Erös (2008); Treer et al. (2008a); Zanella et al. (2008b); Delić et al. (2009); Dumbović et al. (2009); Jelić et al. (2009); Kerestessy et al. (2009); Kurtović et al. (2009); Majnarić (2009); Marijić (2009); Piria et al. (2009); Podrug et al. (2009); Podrug and Raspor (2009); Sofradžija (2009); Treer et al. (2009); Zrnčić et al. (2009a); Bučar et al. (2010); Buj (2010c); Jelić et al. (2010); Ozimec et al. (2010); Perović and Tvrtković (2010); Vardić Smrzlić (2010); Zupančič et al. (2010a); Zupančič et al. (2010b); Matulić et al. (2011); Pavlica et al. (2011); Piria et al. (2011b); Dragun et al. (2012); Jelić et al. (2012); Marčić et al. (2012); Dragun et al. (2013); Filipović Marijić et al. (2013); Krasnići et al. (2013); Delić et al. (2014a); Filipović et al. (2014); Filipović and Raspor (2014); Nedić et al. (2014a); Nedić et al. (2014b); Vukosav et al. (2014); Žutinić et al. (2014); Riđanović et al. (2014); Jelić et al. (2016); Mihailović et al. (2016); Piria et al. (2016a); Piria et al. (2016b); Jelić and Krivek (2017); Mustafić et al. (2017); Pofuk et al. (2017); Simonović et al. (2017b); Nedić et al. (2018); Zuliani et al. (2019)

Remarks: non-native in the Adriatic Basin

\section{Squalius illyricus Heckel \& Kner, 1858}

Names under which the taxon was mentioned: Leuciscus illyricus (Heckel \& Kner, 1858); Leuciscus svallize (Heckel \& Kner, 1858); Squalius albus (Bonaparte, 1838); Squalius cephalus (Linnaeus, 1758); Squalius svallize Heckel \& Kner, 1858

English name: Illyrian chub

Croatian name: ilirski klen 
Range in Croatia: Adriatic Basin - Krka and Cetina River drainages

Literature: Heckel and Kner (1858); Dybowski (1862); Canestrini (1866); Brusina (1872); Canestrini (1874); Brusina (1878); Giglioli (1880); Faber (1883); Kolombatović (1886a); Kolombatović (1886b); Seeley (1886); Kolombatović (1888); Kišpatić (1893); Katurić (1896a); Brusina (1907); Kolombatović (1907); Brusina (1908); Car (1911); Karaman (1923b); Plančić (1950); Taler (1953a); Basioli (1958b); Vinterhalter (1963); Sabioncello (1967); Marko et al. (1968); Vuković and Ivanović (1971); Klašterka (1979); Popović and Habeković (1981); Bojčić et al. (1982); Trrtković (1985); Bianco (1986); Bianco and Knežević (1987); Fašaić et al. (1990); Mrakovčić and Mišetić (1990a); Povž et al. (1990); Economidis and Bănărescu (1991); Popović et al. (1992); Habeković (1993); Habeković (1994b); Popović (1994); Mrakovčić et al. (1995); Crivelli (1996); Maitland and Crivelli (1996); Kottelat (1997); Kerovec (1998); Bogutskaya and Zupančič (1999); Nocita and Vanni (1999); Habeković and Mišetić (2000); Miotti (2002); Mateš (2004); Mustafić (2005); Mrakovčić et al. (2006); Tvrtković and Veen (2006); Valić (2006); Kottelat and Freyhof (2007); Prpa et al. (2007); Valić et al. (2007); Jelić et al. (2008); Marguš (2008); Treer et al. (2008a); Treer et al. (2009); Tvrtković et al. (2009); Perea et al. (2010); Popović (2010); Valić (2010); Vardić Smrzlić (2010); Zupančič et al. (2010a); Zupančič et al. (2010b); Mrakovčić and Ćaleta (2011); Ćaleta et al. (2015); Piria et al. (2016c); Mustafić and Mrakovčić (2017); Pofuk et al. (2017); Jakšić et al. (2019)

Remarks: reports for the Zrmanja River not confirmed, although hybrid individuals $S$. illyricus $\times S$. zrmanjae were found in the Zrmanja River by the authors (unpublished data); stenoendemic to the Krka and Cetina Rivers, endemic to the Adriatic Basin and Croatia

\section{Squalius microlepis Heckel, 1843}

Names under which the taxon was mentioned: Leuciscus microlepis (Heckel, 1843); Leuciscus turskyi microlepis (Heckel, 1843);

English name: Makal dace

Croatian name: makal

Range in Croatia: Adriatic Basin - Vrljika River system, Prološko Blato and Baćinska Lakes, Matica River near Vrgorac

Literature: Heckel (1843); Carrara (1846); Heckel and Kner (1858); Dybowski (1862); Canestrini (1866); Brusina (1872); Canestrini (1874); Brusina (1878); Giglioli (1880); Faber (1883); Katurić (1883); Kolombatović (1886a); Seeley (1886); Kolombatović (1888); Botteri (1891); Kišpatić (1893); Katurić (1896a); Brusina (1907); Katurić 1907); Kolombatović (1907); Car (1911); Karaman (1923a); Karaman (1923b); Vuković and Ivanović (1971); Bojčić et al. (1982); Vuković (1982); Kottelat (1984); Mrakovčić et al. (1996a); Kottelat (1997); Bănărescu et al. (1998); Schneider (1998); Nocita and Vanni (1999); Habeković and Pažur (2000); Mrakovčić et al. (2000a); Miotti (2002); Freyhof et al. (2005a); Freyhof et al. (2006); Mrakovčić et al. (2006); Kottelat and Freyhof (2007); Jelić et al. (2008); Valić et al. (2008); Glamuzina et al. (2010b); Popović (2010); Gabelica et al. (2011); Freyhof (2012); Glamuzina et al. (2013); Duplić (2014); Ćaleta et al. (2015); Piria et al. (2016c); Mustafić and Mrakovčić (2017)

Remarks: reports for Cetina River not confirmed and likely the result of misidentification; endemic to the Adriatic Basin

\section{Squalius squalus (Bonaparte, 1837)}

Names under which the taxon was mentioned: Leuciscus cavedanus Bonaparte, 1838; Leuciscus cephalus (Linnaeus, 1758); Leuciscus cephalus albus Bonaparte, 1838; Leuciscus cephalus cabeda Risso, 1827; Leuciscus cephalus cavedanus; Leuciscus leuciscus (Linnaeus, 1758); Leuciscus svallize (Heckel \& Kner, 1858); Leuciscus zrmanjae (Karaman, 1928); Squalius albus (Bonaparte, 1838); Squalius cavedanus (Bonaparte 1838); Squalius cephalus (Linnaeus, 1758); Squalius svallize Heckel \& Kner, 1858; Squalius tyberinus Bonaparte, 1841

\section{English name: Italian chub}

\section{Croatian name: bijeli klen}

Range in Croatia: Adriatic Basin - middle and lower course of the rivers on the Istrian Peninsula, Krka River

Literature: Bonaparte (1832-1841); Heckel and Kner (1858); Faber (1883); Kolombatović (1886a); Kolombatović (1886b); Seeley (1886); Kolombatović (1888); Botteri (1891); Kišpatić (1893); Steindachner (1895); Katurić (1896c); Langhoffer (1904); Largaiolli (1904); Brusina (1907); Car (1911); Koller (1928); Hafner-Lahorski (1935b); Gridelli (1936); Sabioncello (1967); Vuković and Ivanović (1971); Morović (1976); Žikić and Bertoša (1980); Sofradžija and Hadžiselimović (1981); Bojčić et al. (1982); Vuković (1982); Leiner (1984); Leiner and Popović (1984); Leiner (1985); Bianco and Knežević (1987); Fašaić et al. (1990); Povž et al. (1990); Povž and Sket (1990); Habeković et al. (1993ba); Leiner and Povž (1993); Leiner and Povž (1994); Leiner et al. (1995); Mrakovčić et al. (1995); Mrakovčić et al. (1996a); Leiner (1998b); Bogutskaya and Zupančič (1999); Mrakovčić et al. (2000b); Topić et al. 2000); Mikavica et al. (2001); Povž (2002); Piria et al. (2005b); Mrakovčić et al. (2006); Valić (2006); Kottelat and Freyhof (2007); Šorić (2007); Muhamedagić et al. (2008); Bogutskaya and Zupančič et al. (2010b); Zupančič et al. (2010a); Zupančič et al. (2010b); ); Freyhof (2012); Glamuzina et al. (2013); Bianco (2014a); Bianco (2014b); Ćaleta et al. (2015); Babić (2016); Piria et al. (2016b); Piria et al. (2016c); Mustafić and Mrakovčić (2017)

Remarks: Bogutskaya \& Zupančič (2010) described new species (Sq. janae) to Istrian Peninsula, Adriatic 
Basin (Dragonja, Boljunščica and Pazinčica Rivers). New phylogenetic research (Buj et al. 2019 in press) proved that this is not a valid species but a synonym of $\mathrm{S}$. squalus.

\section{Squalius svallize Heckel \& Kner, 1858}

Names under which the taxon was mentioned: Leuciscus svallize (Heckel \& Kner, 1858)

English name: Neretva chub

Croatian name: svalić

Range in Croatia: Adriatic Basin - Neretva River drainage and Konavosko Field

Literature: Heckel and Kner (1858); Dybowski (1862); Giglioli (1880); Steindachner (1882b); Faber (1883); Katurić (1883); Kolombatović (1886a); Kolombatović (1886b); Seeley (1886); Katurić (1887); Kolombatović (1888); Kišpatić (1893); Katurić (1896a); Kolombatović (1902); Kosić (1903); Langhoffer (1904); Katurić 1907); Kolombatović (1907); Brusina (1908); Car (1911); Karaman (1923b); Koller (1928); Maldini (1936b); Vuković (1961); Vinterhalter (1963); Sabioncello (1967); Vuković and Ivanović (1971); Kolaković (1972); Klašterka (1979); Sofradžija and Hadžiselimović (1981); Bojčić et al. (1982); Leiner (1984); Bianco and Knežević (1987); Mrakovčić and Mišetić (1990a); Povž et al. (1990); Leiner et al. (1995); Mrakovčić et al. (1995); Crivelli (1996); Maitland and Crivelli (1996); Mrakovčić et al. (1996a); Kottelat (1997); Leiner (1998b); Schneider (1998); Tvrtković (1998); Bogutskaya and Zupančič (1999); Nocita and Vanni (1999); Mrakovčić et al. (2000a); Mikavica et al. (2001); Tutman et al. (2002); Tvrtković and Franičević (2002); Aprahamian (2003); Has-Schön et al. (2006); Mrakovčić et al. (2006); Dulčić et al. (2007); Kottelat and Freyhof (2007); Prpa et al. (2007); Jelić et al. (2008); Marguš (2008); Muhamedagić et al. (2008); Treer et al. (2008a); Sofradžija (2009); Treer et al. (2009); Bukvić et al. (2010); Popović (2010); Zupančič et al. (2010a); Freyhof (2012); Piria et al. (2012); Glamuzina et al. (2013); Ćaleta et al. (2015); Piria et al. (2016c); Mustafić and Mrakovčić (2017)

Remarks: reports for the other rivers in the Adriatic Basin, than the two mentioned above, are not confirmed and are likely the result of misidentification; population and taxonomic status of Squalius in the Matica River near Vrgorac and Baćinska Lake is uncertain since there are no recent reports and the presence of $S$. svallize and $S$. microlepis; S. svallize population from the Ljuta River in the Konavosko Field is of hybrid origin (introgressed mitochondrial DNA of S. squalus, Buj et al., 2019, in press); endemic to the Adriatic Basin

\section{Squalius tenellus Heckel, 1843}

Names under which the taxon was mentioned: Leuciscus turskyi tenellus (Heckel, 1843)

English name: Livno masnica

\section{Croatian name: sitnoljuskavi klen}

Range in Croatia: Adriatic Basin - introduced to the Cetina River drainage

Literature: Carrara (1846); Heckel and Kner (1858); Dybowski (1862); Canestrini (1866); Faber (1883); Botteri (1891); Kišpatić (1893); Brusina (1907); Karaman (1923a); Karaman (1923b); Ćurčić (1938); Vuković and Ivanović (1971); Bojčić et al. (1982); Vuković (1982); Habeković and Pažur (2000); Kottelat and Freyhof (2007); Jelić et al. (2008); Freyhof (2012); Ćaleta et al. (2015); Piria et al. (2016c); Mustafić and Mrakovčić (2017)

Remarks: introduced to the Cetina River drainage from Buško Blato Lake (in Bosnia and Herzegovina) through the subterranean system of the Orlovac hydro power plant; reports for the Vrljika River not confirmed and are likely the result of misidentification; endemic to the Adriatic Basin

\section{Squalius zrmanjae Karaman, 1928}

Names under which the taxon was mentioned: Leuciscus svallize (Heckel \& Kner, 1858); Leuciscus svallizze zrmanjae Karaman, 1928; Leuciscus zrmanjae (Karaman, 1928); Squalius cavedanus (Bonaparte 1838); Squalius cephalus (Linnaeus, 1758); Squalius svallize Heckel \& Kner, 1858

English name: Zrmanja chub

žCroatian name: zrmanjski klen

Range in Croatia: Adriatic Basin - Zrmanja and Krka River drainages, introduced to the Ričica River in the Lika region

Literature: Katurić (1896f); Karaman (1928); Karaman (1929); Hafner-Lahorski (1935b); Anonymous (1967); Vuković and Ivanović (1971); Vuković (1982); Leiner (1985); Bianco and Knežević (1987); Kottelat (1997); Schneider (1998); Bogutskaya and Zupančič (1999); Durand et al. (2000); Mrakovčić et al. (2006); Kottelat and Freyhof (2007); Valić et al. (2007); Duplić (2008); Jelić et al. (2008); Perea et al. (2010); Popović (2010); Valić (2010); Vardić Smrzlić (2010); Zupančič et al. (2010a); Zupančič et al. (2010b); Miočić-Stošić and Kovačević (2013); Ćaleta et al. (2015); Jelić et al. (2016); Piria et al. (2016c); Mustafić and Mrakovčić (2017)

Remarks: stenoendemic to the Zrmanja and Krka Rivers, endemic to the Adriatic Basin and Croatia

TELESTES Bonaparte, 1840

73. Telestes croaticus (Steindachner, 1866)

Names under which the taxon was mentioned: Paraphoxinus adspersus (Heckel, 1843); Paraphoxinus croaticus (Steindachner, 1866); Phoxinellus croaticus Steindachner, 1866

English name: Croatian pijor

Croatian name: hrvatski pijor 
Range in Croatia: Adriatic Basin - Jadova, Ričica, Otuča and Obsenica River systems in the Lika region

Literature: Steindachner (1866b); Brusina (1878); Steindachner (1882a); Faber (1883); Seeley (1886); Brusina (1890); Hirc (1897); Hirc (1899b); Hirc (1900a); Hirc (1900b); Langhoffer (1904); Trgovčević (1905a); Trgovčević (1905b); RHZZM (1908); Trgovčević (1908); Car (1911); Vutskits (1918); Karaman (1923a); Karaman (1923b); Poljak (1924); Hirc (1925); Bergleitner (1928); Koller (1928); Trgovčević (1932); Taler (1947); Taler (1951g); Taler (1953a); Vraneš (1966); Anonymous (1967); Sabioncello (1967); Berberović et al. (1971); Guzina and Vuković (1971); Vuković and Ivanović (1971); Sofradžija and Hadžiselimović (1981); Bojčić et al. (1982); Bianco (1986); Povž et al. (1990); Habeković et al. (1992); Mrakovčić et al. (1995); Crivelli (1996); Kottelat (1997); Schneider (1998); Zupančič and Bogutskaya (2000); Miotti (2002); Tvrtković and Franičević (2002); Zupančič and Bogutskaya (2002); Bogutskaya and Zupančič (2003); Ketmaier et al. (2004); Mateš (2004); Freyhof et al. (2006); Mrakovčić et al. (2006); Kottelat and Freyhof (2007); Duplić (2008); Jelić et al. (2008); Palandačić et al. (2010); Perea et al. (2010); Popović (2010); Marčić et al. (2011); Freyhof (2012); Palandačić (2012a); Ćaleta et al. (2015); Ozimec (2015); Jelić et al. (2016); Jelić and Krivek (2017); Buj et al. (2017)

Remarks: reports for the Krbavsko Field, Zrmanja and Zagorska Mrežnica Rivers in the Danube drainage not confirmed and are likely the result of misidentification; locally extinct from the Gacka and Lika Rivers; stenoendemic to the Lika region and endemic to Croatia

\section{Telestes fontinalis (Karaman, 1972)}

Names under which the taxon was mentioned: Paraphoxinus croaticus (Steindachner, 1866); Phoxinellus adspersus fontinalis Karaman, 1972; Phoxinellus fontinalis Karaman, 1972

English name: Krbava dace

Croatian name: krbavski pijor

Range in Croatia: Danube drainage - Krbavsko Field

Literature: Langhoffer (1904); Trgovčević (1905b); Taler (1951e); Taler (1953a); Karaman (1972); Zupančič and Bogutskaya (2000); Tvrtković and Franičević (2002); Zupančič and Bogutskaya (2002); Bogutskaya and Zupančič (2003); Freyhof et al. (2006); Mrakovčić et al. (2006); Kottelat and Freyhof (2007); Duplić (2008); Palandačić et al. (2010); Perea et al. (2010); Popović (2010); Freyhof (2012); Palandačić (2012a); Palandačić (2012b); Ćaleta et al. (2015); Ozimec (2015); Jelić et al. (2016); Jelić and Krivek (2017); Buj et al. (2017)

Remarks: stenoendemic to the Krbavsko Field and endemic to the Danube drainage and Croatia
75. Telestes karsticus Marčić \& Mrakovčić, 2011

Names under which the taxon was mentioned: Leuciscus polylepis (Steindachner, 1866); Paraphoxinus alepidotus (Heckel, 1843); Telestes polylepis Steindachner, 1866

English name: Karst dace

Croatian name: kapelska svijetlica

Range in Croatia: Danube drainage - small creeks in Lug, Jasenačko, Drežničko, Stajničko and Plaško Fields

Literature: Giglioli (1880); Kišpatić (1893); Trgovčević (1905d); Wettstein (1928); Taler (1953a); Vraneš (1966); Hrbek et al. (2004); Ketmaier et al. (2004); Mateš (2004); Freyhof et al. (2006); Zanella et al. (2008a); Buj (2010b); Perea et al. (2010); Popović (2010); Marčić et al. (2011); Freyhof (2012); Marčić (2013); Ćaleta et al. (2015); Ozimec (2015); Marčić et al. (2017a); Marčić et al. (2017b); Buj et al. (2017)

Remarks: stenoendemic to the waters around Ogulin and endemic to the Danube drainage and Croatia

76. Telestes miloradi Bogutskaya, Zupančič, Bogut \& Naseka, 2012

Names under which the taxon was mentioned: Paraphoxinus metohiensis Steindachner, 1901; Paraphoxinus pstrossii Steindachner, 1882; Phoxinellus metohiensis (Steindachner, 1901); Telestes metohiensis (Steindachner, 1901)

English name: Konavle dace

Croatian name: konavoski pijor

Range in Croatia: Adriatic Basin - Konavosko Field

Literature: Steindachner (1901); Kolombatović (1902); Kolombatović (1903); Trgovčević (1905a); Taler (1953a); Taler (1953f); Povž et al. (1990); Kottelat (1997); Zupančič and Bogutskaya (2002); Bogutskaya and Zupančič (2003); Mrakovčić et al. (2006); Bogutskaya et al. (2012); Ćaleta et al. (2015); Jelić and Jelić (2015); Landeka et al. (2015); Ozimec (2015); Mustafić and Mrakovčić (2017); Buj et al. (2017)

Remarks: stenoendemic to the Ljuta River drainage and endemic to the Adriatic Basin and Croatia

\section{Telestes polylepis Steindachner, 1866}

Names under which the taxon was mentioned: Leuciscus polylepis (Steindachner, 1866); Paraphoxinus croaticus (Steindachner, 1866); Phoxinus marsilii Heckel, 1836

\section{English name: Croatian dace}

Croatian name: svijetlica

Range in Croatia: Danube drainage - Dobra and Zagorska Mrežnica Rivers and small creeks and lakes around Josipdol and Ogulin

Literature: Brusina (1878); Steindachner (1866a); Seeley 
(1886); Brusina (1890); Kišpatić (1893); Glowacki (1896); Hirc (1897); Hirc (1900a); Trgovčević (1905d); RHZZM (1908); Car (1911); Vutskits (1918); Karaman (1923a); Karaman (1923b); Anonymous (1925); Bergleitner (1925); Koller (1928); Trgovčević (1932); Taler (1951b); Taler (1953a); Vraneš (1966); Sabioncello (1967); Vuković and Ivanović (1971); Conci and Michelangeli (1974); Bojčić et al. (1982); Tvrtković (1985); Bianco (1986); Mrakovčić and Mišetić (1990a); Povž et al. (1990); Economidis and Bănărescu (1991); Mrakovčić et al. (1995); Crivelli (1996); Maitland and Crivelli (1996); Kottelat (1997); Bănărescu et al. (1998); Miotti (2002); Tvrtković and Franičević (2002); Mateš (2004); Mrakovčić et al. (2006); Tvrtković and Veen (2006); Kottelat and Freyhof (2007); Duplić (2008); Jalžić et al. (2008); Valić et al. (2008); Buj (2010b); Perea et al. (2010); Popović (2010); Marčić et al. (2011); Freyhof (2012); Marčić (2013); Ćaleta et al. (2015); Ozimec (2015); Buj et al. (2017)

Remarks: stenoendemic to the waters around Ogulin, endemic to the Danube drainage and Croatia; reports for the Krka and Čikola Rivers not confirmed and are likely the result of misidentification

\section{Telestes souffia (Risso, 1827)}

Names under which the taxon was mentioned: Leuciscus agassizii Valenciennes, 1844; Leuciscus souffia Risso, 1827; Leuciscus souffia agassizi Valenciennes, 1844; Telestes agassizii (Valenciennes, 1844)

English name: Souffia

Croatian name: blistavac

Range in Croatia: Danube drainage - upper part of the Sava River with small tributaries

Literature: Heckel and Kner (1858); Glowacki (1885); Anonymous (1886); Kišpatić (1893); Glowacki (1896); Mojsisovics (1897); Lang (1911); Vutskits (1918); Vuković and Ivanović (1971); Crivelli (1996); Mrakovčić et al. (1996b); Habeković et al. (1997); Mrakovčić et al. (2006); Tvrtković and Veen (2006); Kottelat and Freyhof (2007); Treer et al. (2008a); Treer et al. (2009); Jelić et al. (2012); Ozimec (2015); Vucić et al. (2017); Buj et al. (2017)

\section{Telestes turskyi (Heckel 1843)}

Names under which the taxon was mentioned: Leuciscus polylepis (Steindachner, 1866); Leuciscus turskyi (Heckel, 1843); Squalius turskyi Heckel, 1843; Squalius tursckyi

English name: Tursky dace

Croatian name: turski klen

Range in Croatia: Adriatic Basin - Krka River drainage

Literature: Heckel (1843); Bonaparte (1845); Bonaparte (1846); Carrara (1846); Heckel and Kner (1858); Dybowski (1862); Canestrini (1866); Giglioli (1880); Faber (1883); Kolombatović (1886b); Seeley (1886); Botteri (1891);
Kišpatić (1893); Brusina (1907); Car (1911); Karaman (1923a); Karaman (1923b); Pešić (1961); Sabioncello (1967); Vuković and Ivanović (1971); Sofradžija and Hadžiselimović (1981); Bojčić et al. (1982); Tvrtković (1985); Bianco (1986); Povž et al. (1990); Crivelli (1996); Kottelat (1997); Bănărescu et al. (1998); Nocita and Vanni (1999); Miotti (2002); Tvrtković and Franičević (2002); Salzburger et al. (2003); Hrbek et al. (2004); Ketmaier et al. (2004); Freyhof et al. (2006); Mrakovčić et al. (2006); Kottelat and Freyhof (2007); Jelić et al. (2008); Valić et al. (2008); Zanella et al. (2008a); Sofradžija (2009); Perea et al. (2010); Popović (2010); Freyhof (2012); Mihinjač et al. (2014); Ćaleta et al. (2015); Landeka et al. (2015); Ozimec (2015); Mustafić and Mrakovčić (2017); Buj et al. (2017)

Remarks: stenoendemic to the Krka River drainage and endemic to the Adriatic Basin and Croatia

\section{Telestes ukliva (Heckel, 1843)}

Names under which the taxon was mentioned: Leuciscus souffia Risso, 1827; Leuciscus souffia agassizi Valenciennes, 1844; Leuciscus souffia muticellus Bonaparte, 1837; Leuciscus ukliva (Heckel, 1843); Paraphoxinus alepidotus (Heckel, 1843); Squalius ukliva Heckel, 1843; Telestes souffia (Risso, 1827)

English name: Cetina dace

Croatian name: cetinska ukliva

Range in Croatia: Adriatic Basin - Čikola River drainage

Literature: Heckel (1843); Bonaparte (1845); Bonaparte (1846); Carrara (1846); Heckel and Kner (1858); Dybowski (1862); Canestrini (1866); Giglioli (1880); Faber (1883); Kolombatović (1886a); Kolombatović (1886b); Seeley (1886); Kolombatović (1888); Botteri (1891); Kišpatić (1893); Brusina (1907); Car (1911); Karaman (1923a); Karaman (1923b); Taler (1953a); Sabioncello (1967); Marko et al. (1968); Vuković and Ivanović (1971); Popović and Habeković (1981); Bojčić et al. (1982); Leiner and Popović (1984); Tvrtković (1985); Bianco (1986); Povž et al. (1990); Economidis and Bănărescu (1991); Habeković (1993); Habeković (1994b); Mrakovčić et al. (1995); Crivelli (1996); Kottelat (1997); Bănărescu et al. (1998); Kerovec (1998); Schneider (1998); Nocita and Vanni (1999); Habeković and Mišetić (2000); Faber (1883); Miotti (2002); Zanella (2003); Ketmaier et al. (2004); Mustafić (2005); Mrakovčić et al. (2006); Kottelat and Freyhof (2007); Prpa et al. (2007); Duplić (2008); Jelić et al. (2008); Treer et al. (2008a); Valić et al. (2008); Zanella et al. (2008a); Treer et al. (2009); Tvrtković et al. (2009); Zanella et al. (2009c); Perea et al. (2010); Popović (2010); Valić et al. (2010); Marčić et al. (2011); Mrakovčić and Ćaleta (2011); Freyhof (2012); Ćaleta et al. (2015); Ozimec (2015); Mustafić and Mrakovčić (2017); Buj et al. (2017)

Remarks: reports for the lakes near Imotski not confirmed and are likely the result of misidentification; stenoendemic to the Cetina River drainage and endemic to the Adriatic 
Basin and Croatia

VIMBA Fitzinger, 1873

81. Vimba vimba (Linnaeus, 1758)

Names under which the taxon was mentioned: Abramis melanops (Heckel, 1837); Abramis vimba (Linnaeus, 1758); Vimba elongata (Valenciennes, 1844); Vimba vimba carinata (Pallas, 1814)

English name: Zarte

Croatian name: nosara

Range in Croatia: Danube drainage - middle and lower course of rivers

Literature: Heckel and Kner (1858); Steindachner (1866b); Jurinac (1884); Glowacki (1885); Kesterčanek (1886); Seeley (1886); Brusina (1892); Brusina (1893); Kišpatić (1893); Glowacki (1896); Mojsisovics (1897); Langhoffer (1904); RHZZM (1908); Ćurčić (1910); Vutskits (1918); Plančić (1923b); Maldini (1935a); Rotarides (1944); Sabioncello (1967); Vuković and Ivanović (1971); Mikuska (1979); Sofradžija and Hadžiselimović (1981); Bojčić et al. (1982); Veljović (1982); Mikuska (1983a); Mikuska (1983b); Mikuska (1984); Vrenk (1984); Veljović (1985); Habeković et al. (1986); Lelek (1987); Habeković et al. (1990); Habeković and Popović (1991); Mrakovčić (1992); Mrakovčić et al. (1996b); Habeković et al. (1997); HonsigErlenburg et al. (1997); Leiner (1998a); Majer (1998); Povž et al. (1998); Mustafić (2001); Jakirčević (2005); Mustafić (2005); Mrakovčić et al. (2006); Ćaleta (2007); Kottelat and Freyhof (2007); Piria (2007); Prpa et al. (2007); Sallai and Mrakovčić (2007); Sallai and Kontos (2008); Treer et al. (2008a); Delić et al. (2009); Dumbović et al. (2009); Kerestessy et al. (2009); Sofradžija (2009); Treer et al. (2009); Ozimec et al. (2010); Jelić et al. (2012); Delić et al. (2014a); Nedić et al. (2014a); Nedić et al. (2014b); Riđanović et al. (2014); Piria et al. (2016a); Simonović et al. (2017b); Nedić et al. (2018)

\subsubsection{FAMILY: TINCIDAE Jordan, 1878}

TINCA Cuvier, 1816

82. Tinca tinca (Linnaeus, 1758)

Names under which the taxon was mentioned: Cyprinus tinca Linnaeus, 1758; Tinca chrysitis Fitzinger, 1832; Tinca vulgaris Fleming, 1828

English name: Tench

Croatian name: linjak

Range in Croatia: Danube drainage - middle and lower course of rivers and lakes; Adriatic Basin - introduced to rivers and reservoirs

Literature: Fortis (1771); Carrara (1846); Heckel and Kner (1858); Steindachner (1866b); Šloser (1870); Brusina (1878); Jurinac (1880); Sebišanović (1880); Sebišanović
(1881); Mojsisovics (1883); Jurinac (1884); Mojsisovics (1884); Glowacki (1885); Anonymous (1886); Kesterčanek (1886); Seeley (1886); Hefele (1889); Sebišanović (1889a); Sebišanović (1890); Brusina (1892); Brusina (1893); Dudan (1893); Kišpatić (1893); Kolombatović (1893); Glowacki (1896); Hirc (1897); Hirc (1899d); Hirc (1900b); Horvat (1900c); Hirc (1902d); Largaiolli (1904); Ivakić (1905); Anonymous (1906d); Katurić 1907); RHZZM (1908); Anonymous (1910b); Ćurčić (1910); Hirc (1911); Karaman (1923b); Plančić (1923b); Karaman (1928); Rössler (1928); Kaitner (1929a); Anonymous (1931c); Fink (1932a); Thaller (1932); Gridelli (1936); Maldini (1936b); Maldini (1938c); Fijan (1948a); Morović (1948); Taler (1948c); Taler (1948e); Fijan (1949); Franić (1949); Fijan (1951a); Basioli (1952); Glavan (1952); Plančić (1952c); Plančić (1952d); Taler (1953d); Taler (1953a); Škovrlj (1953a); Pešić (1955); Plančić (1955); Fijan (1956); Grce (1956); Asaj and Šalić (1957); Basioli (1957b); Grce (1957); Morović (1962); Morović (1963); Morović (1964a); Sabioncello et al. (1964); Vraneš (1966); Habeković (1967); Sabioncello (1967); Zobundžija (1968); Sabioncello et al. (1969); Sabioncello (1971a); Vuković and Ivanović (1971); Pogrmilović (1972); Munjko (1978); Klašterka (1979); Mikuska (1979); Sofradžija and Hadžiselimović (1981); Bojčić et al. (1982); Veljović (1982); Mikuska (1983a); Mikuska (1983b); Leiner (1984); Mikuska (1984); Mikuska and Mamić (1984); Ocvirk (1984); Pompe-Gotal (1984); Vrenk (1984); Leiner (1985); Mikuska (1985); Ocvirk (1985); Tvrtković (1985); Habeković et al. (1986); Delić (1987); Lelek (1987); Turk et al. (1987); Mišetić et al. (1989); Fašaić et al. (1990); Habeković et al. (1990); Mrakovčić and Mišetić (1990a); Habeković (1991); Mrakovčić (1992); Delić (1993); Host (1993); Leiner and Povž (1993); Kolar-Dimitrijević (1994); Leiner and Povž (1994); Leiner et al. (1995); Mrakovčić et al. (1995); Povž (1995); Maitland and Crivelli (1996); Mrakovčić et al. (1996a); Mrakovčić et al. (1996b); Leiner (1998a); Leiner (1998b); Majer (1998); Mrakovčić (1998); Povž et al. (1998); Treer et al. (1998); Tvrtković (1998); Brylińska et al. (1999); Mrakovčić et al. (2000a); Mrakovčić et al. (2000b); Zanella et al. (2000); Topić Popović et al. (2000); Mustafić (2001); Mrakovčić et al. (2002); Bakota et al. (2003); Jugović (2004); Bašić (2005); Jakirčević (2005); Mustafić (2005); Pažur (2005); Suić (2005); HasSchön et al. (2006); Mrakovčić et al. (2006); Valić (2006); Knežević (2007); Kottelat and Freyhof (2007); Prpa et al. (2007); Sallai and Mrakovčić (2007); Valić et al. (2007); Marguš (2008); Sallai and Kontos (2008); Sevcsik and Erös (2008); Suić et al. (2008); Treer et al. (2008a); Dumbović et al. (2009); Jelić et al. (2009); Sofradžija (2009); Treer et al. (2009); Buj (2010c); Bukvić et al. (2010); Ozimec et al. (2010); Valić (2010); Vardić Smrzlić (2010); Jelić et al. (2012); Marčić et al. (2012); Glamuzina et al. (2013); Marr et al. (2013); Delić et al. (2014a); Babić (2016); Jelić et al. (2016); Jelić and Krivek (2017); Lujić et al. (2017); Mustafić et al. (2017); Pofuk et al. (2017); Nedić et al. (2018)

Remarks: non-native in the Adriatic Basin 


\subsubsection{FAMILY: XENOCYPRIDIDAE Gunther, 1868}

CTENOPHARYNGODON Steindachner, 1866

83. Ctenopharyngodon idella (Valenciennes, 1844)

English name: Grass carp

Croatian name: amur

Range in Croatia: Danube drainage: middle and lower course of rivers, lakes and reservoirs; Adriatic Basin reservoirs

Literature: Sabioncello (1967); Vinterhalter (1967); Fijan and Vojta (1969); Vinterhalter (1969); Marko and Stoisavljević (1972); Pogrmilović (1972); Fijan (1976a); Mikuska (1979); Bojčić et al. (1982); Veljović (1982); Meštrov and Justić (1983); Mikuska (1983a); Mikuska (1983b); Mikuska (1984); Mikuska and Mamić (1984); Pompe-Gotal (1984); Leiner (1985); Mikuska (1985); Delić (1987); Safner (1987); Turk et al. (1987); Delić (1989); Delić (1993); Turk and Debeljak (1995); Mihaljević et al. (1996); Mrakovčić et al. (1996b); Majer (1998); Treer et al. (1998); Mustafić (2001); Piria et al. (2004); Jakirčević (2005); Mustafić (2005); Pažur (2005); Mrakovčić et al. (2006); Prpa et al. (2007); Sallai and Mrakovčić (2007); Sallai and Kontos (2008); Treer et al. (2008a); Sofradžija (2009); Treer et al. (2009); Ozimec et al. (2010); Jelić et al. (2012); Marčić et al. (2012); Marr et al. (2013); Zrnčić et al. (2013); Nedić et al. (2014a); Nedić et al. (2014b); Povž and Gregori (2014); Riđanović et al. (2014); Jelić et al. (2016); Piria et al. (2016b); Mustafić et al. (2017); Pofuk et al. (2017)

Remarks: non-native species

HYPOPHTHALMICHTHYS Bleeker, 1860

84. Hypophthalmichthys molitrix (Valenciennes, 1844)

English name: Silver carp

Croatian name: bijeli glavaš

Range in Croatia: Danube drainage - lower course of rivers and reservoirs; Adriatic Basin - Neretva River and some reservoirs

Literature: Vinterhalter (1967); Fijan and Vojta (1969); Vuković and Ivanović (1971); Pogrmilović (1972); Mikuska (1979); Meštrov and Justić (1983); Mikuska (1983a); Mikuska (1983b); Mikuska (1984); Pompe-Gotal (1984); Vrenk (1984); Delić (1987); Turk et al. (1987); Delić (1989); Homen et al. (1991); Harka (1992); Delić (1993); Turk and Debeljak (1995); Mrakovčić et al. (1996b); Treer et al. (1998); Jakirčević (2005); Mustafić (2005); Pažur (2005); Mrakovčić et al. (2006); Sallai and Mrakovčić (2007); Sallai and Kontos (2008); Jelić et al. (2009); Buj (2010c); Ozimec et al. (2010); Jelić et al. (2012); Glamuzina et al. (2013); Zrnčić et al. (2013); Piria et al. (2016b); Piria et al. (2016c); Mustafić et al. (2017); Pofuk et al. (2017); Piria et al. (2018)
85. Hypophthalmichthys nobilis (Richardson, 1845)

Names under which the taxon was mentioned: Aristichthys nobilis (Richardson 1845)

English name: Bighead carp

Croatian name: sivi glavaš

Range in Croatia: Danube drainage - lower course of rivers and reservoirs; Adriatic Basin - Neretva, Cetina and Lika Rivers and some reservoirs

Literature: Pogrmilović (1972); Vrenk (1984); Delić (1987); Turk et al. (1987); Delić (1989); Mišetić et al. (1989); Turk and Debeljak (1995); Zrnčić (1995); Mrakovčić et al. (1996b); Majer (1998); Simonović (2001); Piria et al. (2004); Mustafić (2005); Pažur (2005); Mrakovčić et al. (2006); Sallai and Mrakovčić (2007); Sallai and Kontos (2008); Ozimec et al. (2010); Jelić et al. (2012); Marčić et al. (2012); Jelkić and Opačak (2013); Jelić et al. (2016); Piria et al. (2016b); Piria et al. (2016c); Mustafić et al. (2017); Pofuk et al. (2017); Piria et al. (2018)

Remarks: non-native species

\section{ORDER: CHARACIFORMES}

FAMILY: SERRASALMIDAE Bleeker, 1859

PIARACTUS Eigenmann, 1903

UI3 Piaractus brachypomus (Cuvier, 1818)

English name: Pirapitinga

Croatian name: Pirapitinga

Range in Croatia: Danube drainage - two specimens reported in the lower Drava River near Osijek

Literature: Ćaleta et al. (2011a); Piria et al. (2016b); Piria et al. (2018)

Remarks: non-native species; individual reports without the establishment of a self-sustaining population

\subsection{ORDER: SILURIFORMES}

\section{FAMILY: MOCHOKIDAE Jordan, 1923}

SYNODONTIS Cuvier, 1816

UI4 Synodontis eupterus Boulenger, 1901

English name: Featherfin squeaker

Croatian name: krilasti som

Range in Croatia: Adriatic Basin - Mala Neretva (Neretva River drainage) near Opuzen

Literature: Dulčić et al. (2018)

Remarks: non-native, aquarium species; one recorded individual, no self-sustaining populations established 


\subsubsection{FAMILY: SILURIDAE Rafinesque, 1815}

SILURUS Linnaeus, 1758

86. Silurus glanis Linnaeus, 1758

English name: Wels catfish

Croatian name: som

Range in Croatia: Danube drainage - middle and lower course of rivers and lakes; Adriatic Basin -introduced to Vransko Lake near Biograd and the Lika River

Literature: Heckel and Kner (1858); Šloser (1870); Brusina (1878); Jurinac (1880); Sebišanović (1880); Borne (1881); Sebišanović (1881); Mojsisovics (1883); Mojsisovics (1884); Jurinac (1884); Glowacki (1885); Anonymous (1886); Kesterčanek (1886); Seeley (1886); Jurinac (1887); Šoštarić (1888); Hefele (1889); Sebišanović (1890); Brusina (1892); Franke (1892); Anonymous (1893b); Brusina (1893); Dudan (1893); Kišpatić (1893); Flögel (1894); Glowacki (1896); Hirc (1896); Hirc (1897); Anonymous (1899c); Steuer (1899); Aue (1900); Anonymous (1901); Horvat (1901); Ribić (1901); Ivakić (1905); Peheim (1905); Anonymous (1906d); Anonymous (1906e); RHZZM (1908); Peheim (1909); Ćurčić (1910); Kempf (1910); Vutskits (1918); Plančić (1920); Plančić (1923a); Anonymous (1925); Anonymous (1926); Anonymous (1927b); Bajić (1928); Rössler (1928); Anonymous (1930b); Anonymous (1930f); Anonymous (1930h); Anonymous (1931a); Anonymous (1931c); Anonymous (1931g); Fink (1932a); Fink (1932b); Maldini (1933); Ćurčić (1933); Trojanović (1934); Anonymous (1935e); Bek (1935); Jedlička (1935); Samobor (1935); Anonymous (1937b); Maldini (1937); Anonymous (1948d); Taler (1945a); Anonymous (1946a); Anonymous (1947a); Anonymous (1947c); Anonymous (1948b); Fijan (1948a); Taler (1948f); Fijan (1950); HafnerLahorski (1950a); Taler (1951e); Anonymous (1952c); Marjanović (1954); Pešić (1955); Plančić (1955); Fijan (1956); Grce (1956); Asaj and Šalić (1957); Grce (1957); Jagodić (1957); Anonymous (1959); Grce (1959); Smlatić (1961); Livojević (1962b); Livojević (1962a); Morović (1962); Hadžić (1964b); Morović (1964a); Sabioncello et al. (1964); Hadžić (1965); Salihćehajić (1965); Stepanek (1965); Bikić (1966); Habeković (1967); Hadžić (1967); Morović (1967); Sabioncello (1967); Zobundžija (1968); Anonymous (1969b); Sabioncello et al. (1969); Vuković and Ivanović (1971); Munjko (1972); Ristić (1972b); Vrebac (1972); Herga (1968); Pogrmilović (1972); Fodolović (1973); Mišković (1973); Fijan (1976a); Čanadjija (1977); Munjko (1978); Klašterka (1979); Mikuska (1979); Bojčić et al. (1982); Veljović (1982); Mikuska (1983a); Mikuska (1983b); Delić (1984); Mikuska (1984); Pompe-Gotal (1984); Ržaničanin et al. (1984b); Treer et al. (1984); Tvrtković (1985); Veljović (1985); Delić (1987); Habeković (1987); Lelek (1987); Treer (1987); Turk et al. (1987); Delić (1989); Treer (1989); Treer et al. (1989); Habeković et al. (1990); Kažić et al. (1990); Homen et al. (1991); Popović (1991); Treer et al. (1991); Mrakovčić (1992); Delić (1993);
Pažur (1993); Crivelli (1995); Mrakovčić et al. (1995); Zrnčić (1995); Mihaljević et al. (1996); Mrakovčić et al. (1996b); Habeković et al. (1997); Leiner (1998a); Majer (1998); Povž et al. (1998); Treer et al. (1998); Zanella et al. (2000); Mustafić (2001); Mrakovčić et al. (2002); Delić et al. (2003a); Leiner (2003); Mateš (2004); Piria et al. (2004); Jakirčević (2005); Mustafić (2005); Pažur (2005); Petrinec et al. (2005); Ćaleta (2007); Kottelat and Freyhof (2007); Prpa et al. (2007); Sallai and Mrakovčić (2007); Sallai and Kontos (2008); Sevcsik and Erös (2008); Suić et al. (2008); Treer et al. (2008a); Delić et al. (2009); Dumbović et al. (2009); Sofradžija (2009); Treer et al. (2009); Bučar et al. (2010); Jelić et al. (2010); Jelkić et al. (2010); Ozimec et al. (2010); Labak (2011); Matulić et al. (2011); Treer et al. (2011); Jelić et al. (2012); Glamuzina et al. (2013); Jelkić and Opačak (2013); Marr et al. (2013); Šanda et al. (2013); Zrnčić et al. (2013); Delić et al. (2014a); Nedić et al. (2014a); Nedić et al. (2014b); Riđanović et al. (2014); Jelić et al. (2016); Piria et al. (2016b); Piria et al. (2016c); Mustafić et al. (2017); Pofuk et al. (2017); Simonović et al. (2017b); Nedić et al. (2018)

Remarks: non-native in the Adriatic Basin

\section{FAMILY: CLARIIDAE Bonaparte, 1846}

CLARIAS Scopoli, 1777

UI5 Clarias gariepinus Burchell, 1822

English name: North African catfish

Croatian name: afrički som

Range in Croatia: Danube drainage - introduced locally

Literature: Piria et al. (2018)

Remarks: non-native species; introduced to the Draganići fish farm for aquaculture purposes (Recirculation Aquaculture Systems) without a self-sustaining population

\subsubsection{FAMILY: ICTALURIDAE Gill, 1861}

AMEIURUS Rafinesque, 1820

87. Ameiurus melas (Rafinesque, 1820)

Names under which the taxon was mentioned: Ameiurus nebulosus (Lesueur, 1819); Ictalurus melas (Rafinesque, 1820); Ictalurus nebulosus (Lesueur, 1819); Ictalurus punctatus (Rafinesque, 1818)

English name: Black bullhead

Croatian name: crni somić

Range in Croatia: Danube drainage - middle and lower course of the rivers and backwaters; Adriatic Basin rivers, reservoirs and lakes

Literature: Veljović (1982); Harka (1992); Habeković (1993); Crivelli (1995); Majer (1998); Schneider (1998); Mateš (2004); Bošnir et al. (2005); Jakirčević (2005); Mustafić (2005); Mrakovčić et al. (2006); Ćaleta (2007); 
Duplić (2008); Sallai and Kontos (2008); Delić et al. (2009); Jelić et al. (2009); Jelić et al. (2010); Ozimec et al. (2010); Mrakovčić and Ćaleta (2011); Jelić et al. (2012); Glamuzina et al. (2013); Marr et al. (2013); Povž and Gregori (2014); Piria et al. (2017); Nedić et al. (2018); Piria et al. (2018)

Remarks: non-native species; older reports of $A$. nebulosus are misidentified and belong to $A$. melas or $A$. melas recently replaced $A$. nebulosus in freswaters of Croatia

\section{Ameiurus nebulosus (Lesueur, 1819)}

Names under which the taxon was mentioned: Ictalurus nebulosus (Lesueur, 1819)

English name: Brown bullhead

Croatian name: smeđi somić

Range in Croatia: Adriatic Basin - Neretva River drainage

Literature: Anonymous (1906d); Anonymous (1907d); Anonymous (1907b); Anonymous (1907c); Anonymous (1910a); Hirc (1911); Anonymous (1925); Rössler (1928); Anonymous (1931c); Ivančić (1946); Karaman (1952); Sabioncello (1967); Sabioncello (1971b); Vuković and Ivanović (1971); Čanadjija (1977); Klašterka (1979); Mikuska (1979); Bojčić et al. (1982); Mikuska (1983b); Mikuska (1983a); Delić (1984); Mikuska (1984); Vrenk (1984); Bojčić (1987); Delić (1989); Fijan et al. (1989); Habeković et al. (1990); Martinović et al. (1992); Delić (1993); Zrnčić (1995); Mrakovčić et al. (1996b); Delić et al. (1997); Kerovec (1998); Leiner (1998a); Leiner (1998b); Schneider (1998); Zanella et al. (2000); Mustafić (2001); Mrakovčić et al. (2002); Bakota et al. (2003); Bošnir et al. (2003); Delić et al. (2003a); Jugović (2004); Pažur (2005); Mrakovčić et al. (2006); Bošnir et al. (2007); Dulčić et al. (2007); Prpa et al. (2007); Sallai and Mrakovčić (2007); Sallai and Kontos (2008); Sevcsik and Erös (2008); Treer et al. (2008a); Treer et al. (2009); Sofradžija (2009); Bukvić et al. (2010); Vardić Smrzlić (2010); Jelić et al. (2012); Glamuzina et al. (2013); Jelkić and Opačak (2013); Nedić et al. (2014a); Nedić et al. (2014b); Riđanović et al. (2014); Povž and Gregori (2014); Povž and Gregori (2014); Piria et al. (2016b); Nedić et al. (2018); Piria et al. (2018)

Remarks: non-native species; older reports of $A$. nebulosus are misidentified and belong to $A$. melas or A. melas recently replaced $A$. nebulosus in freswaters of Croatia

ICTALURUS Rafinesque, 1820

UI6 Ictalurus punctatus (Rafinesque, 1818)

English name: Channel catfish

Croatian name: kanalski somić

Range in Croatia: Danube drainage - introduced locally to fishponds

Literature: Sabioncello (1971b); Fijan et al. (1989)
Remarks: non-native species; introduced to the Našice fishpond, though breeding was unsuccessful

\subsection{ORDER: ESOCIFORMES}

\subsubsection{FAMILY: ESOCIDAE Rafinesque, 1815}

\section{ESOX Linnaeus, 1758}

89. Esox lucius Linnaeus, 1758

English name: Northern pike

Croatian name: štuka

Range in Croatia: Danube drainage - middle and lower course of rivers and lakes; Adriatic Basin - introduced to Vransko Lake near Biograd, Vransko Lake on Cres Island, Ponikve Lake on Krk Island, Mirna, Gacka, Lika, Cetina and Neretva Rivers

Literature: Fortis (1771); Taube (1777); Heckel and Kner (1858); Steindachner (1866b); Šloser (1870); Jurinac (1880); Sebišanović (1880); Borne (1881); Sebišanović (1881); Faber (1883); Mojsisovics (1883); Jurinac (1884); Mojsisovics (1884); Glowacki (1885); Anonymous (1886); Kesterčanek (1886); Seeley (1886); Jurinac (1887); Hefele (1889); Sebišanović (1889c); Sebišanović (1889a); Sebišanović (1890); Brusina (1892); Franke (1892); Anonymous (1893a); Anonymous (1893d); Brusina (1893); Dudan (1893); Kišpatić (1893); Anonymous (1894a); Anonymous (1894c); Anonymous (1894f); Anonymous (1895); Anonymous (1896a); Glowacki (1896); Hirc (1896); Brusina (1897); Hirc (1897); Anonymous (1898a); Hirc (1898); Hirc (1900a); Hirc (1900b); Langhoffer (1904); Ivakić (1905); Anonymous (1907a); Brusina (1907); RHZZM (1908); Trgovčević (1908); Anonymous (1909a); Anonymous (1910b); Ćurčić (1910); Franić (1910); Kempf (1910); Car (1911); Vutskits (1918); Plančić (1920); Karaman (1923b); Plančić (1923a); Plančić (1923b); Anonymous (1925); Hirc (1925); Bajić (1927); Rössler (1928); Wettstein (1928); Anonymous (1929a); Anonymous (1930b); Anonymous (1930c); Anonymous (1930f); Anonymous (1930g); Anonymous (1931b); Anonymous (1931c); Anonymous (1931g); Kaitner (1931); Fink (1932a); Kaitner (1932); Krajačić (1932); Rössler (1932); Trgovčević (1932); Ćurčić (1933); Trojanović (1934); Bek (1935); Hafner-Lahorski (1935c); Jedlička (1935); Samobor (1935); Anonymous (1936b); Gridelli (1936); Maldini (1936a); Anonymous (1937b); Maldini (1937); Maldini (1938b); Taler (1945a); Anonymous (1946a); Kapac (1948); Taler (1948c); Taler (1948e); Franić (1949); Hafner-Lahorski (1950a); Taler (1950b); Taler (1951g); Uzelac (1952a); Plančić et al. (1953); Taler (1953a); Taler (1953d); Taler (1953c); Taler (1953a); Marjanović (1954); Pešić (1955); Basioli (1957b); Jagodić (1957); Smlatić (1961); Winterhalter (1964); Hadžić (1964a); Sabioncello (1967); Pažur (1968a); Zobundžija (1968); Sabioncello et al. (1969); Vrebac (1970); Vuković and Ivanović (1971); Munjko (1972); Vrebac (1972); Štefanac (1973); Munjko (1978); Klašterka (1979); Mikuska (1979); Pažur (1979); 
Sofradžija and Hadžiselimović (1981); Bojčić et al. (1982); Veljović (1982); Vuković (1982); Mikuska (1983a); Mikuska (1983b); Delić (1984); Mikuska (1984); Mikuska and Mamić (1984); Ocvirk (1984); Pompe-Gotal, (1984); Vrenk (1984); Delić (1985); Leiner (1985); Mikuska (1985); Ocvirk (1985); Tvrtković (1985); Veljović (1985); Habeković et al. (1986); Lelek (1987); Delić (1989); Treer (1989); Habeković et al. (1990); Habeković and Popović (1991); Homen et al. (1991); Popović (1991); Mrakovčić (1992); Ocvirk (1992); Delić (1993); Host (1993); Leiner and Povž (1993); Pažur (1993); Leiner and Povž (1994); Leiner et al. (1995); Povž (1995); Mihaljević et al. (1996); Mrakovčić et al. (1996b); Biber (1997); Habeković and Pažur (1998); Leiner (1998a); Majer (1998); Povž et al. (1998); Treer et al. (1998); Holčík and Delić (2000); Topić Popović et al. (2000); Zanella et al. (2000); Mustafić (2001); Simonović (2001); Mrakovčić et al. (2002); Bakota et al. (2003); Delić et al. (2003a); Leiner (2003); Jugović (2004); Piria et al. (2004); Bašić (2005); Jakirčević (2005); Mustafić (2005); Pažur (2005); Petrinec et al. (2005); Suić (2005); Mrakovčić et al. (2006); Ćaleta (2007); Ivelić et al. (2007); Knežević (2007); Kottelat and Freyhof (2007); Prpa et al. (2007); Sallai and Mrakovčić (2007); Sallai and Kontos (2008); Sevcsik and Erös (2008); Suić et al. (2008); Treer et al. (2008a); Delić et al. (2009); Dumbović et al. (2009); Jelić et al. (2009); Kerestessy et al. (2009); Treer et al. (2009); Tvrtković et al. (2009); Sofradžija (2009); Bučar et al. (2010); Buj (2010c); Jelić et al. (2010); Jelkić et al. (2010); Ozimec et al. (2010); Vardić Smrzlić (2010); Labak (2011); Matulić et al. (2011); Mrakovčić and Ćaleta (2011); Jelić et al. (2012); Marčić et al. (2012); Marr et al. (2013); Šanda et al. (2013); Valić et al. (2013); Zrnčić et al. (2013); Delić et al. (2014a); Nedić et al. (2014a); Nedić et al. (2014b); Riđanović et al. (2014); Babić (2016); Jelić et al. (2016); Piria et al. (2016b); Piria et al. (2016c); Mustafić et al. (2017); Pofuk et al. (2017); Simonović et al. (2017b); Nedić et al. (2018); Piria et al. (2018); Zuliani et al. (2019)

Remarks: non-native in the Adriatic Basin

\subsubsection{FAMILY: UMBRIDAE Bonaparte, 1845}

UMBRA Walbaum, 1792

90. Umbra krameri Walbaum, 1792

Names under which the taxon was mentioned: Umbra canina Károli, 1882

English name: European mudminnow

Croatian name: crnka

Range in Croatia: Danube drainage - lower course and backwaters of the Mura, Drava and Danube Rivers, Žutica Forest and upper course of the Odra River in the Sava River system

Literature: Heckel and Kner (1858); Mojsisovics (1887); Kišpatić (1893); Mojsisovics (1897); Brusina (1899); Langhoffer (1904); RHZZM (1908); Maldini (1936b);
Taler (1951b); Anonymous (1953a); Pavletić (1954); Pešić (1955); Sabioncello (1967); Vuković and Ivanović (1971); Mikuska (1979); Mikuska (1983b); Povž (1984); Mrakovčić and Kerovec (1990); Leiner (1995); Povž (1995); Wanzenböck (1995); Mrakovčić et al. (1996b); Delić et al. (1997); Zanella et al. (2000); Mustafić (2001); Leiner (2003); Pažur (2005); Sallai and Kontos (2005); Mrakovčić et al. (2006); Sallai and Mrakovčić (2007); Sallai and Kontos (2008); Freyhof (2012); Jelić et al. (2012); Marić et al. (2017)

\subsection{ORDER: SALMONIFORMES}

\subsubsection{FAMILY: SALMONIDAE Schinz, 1822}

COREGONUS Lacepède, 1803

91. Coregonus lavaretus (Linnaeus, 1758)

Names under which the taxon was mentioned: Coregonus lavaretus maraena (Bloch, 1779)

English name: European whitefish

Croatian name: velika ozimica

Range in Croatia: Danube drainage - Drava River; Adriatic Basin- introduced to reservoirs on the Cetina River

Literature: Habeković (1978a); Vrenk (1984); Jevtić (1991); Habeković (1993); Crivelli (1995); Mrakovčić et al. (1996b); Kerovec (1998); Pažur (2005); Tvrtković et al. (2009); Schneider (1998); Mrakovčić et al. (2006); Mrakovčić and Ćaleta (2011); Jelić et al. (2012); Povž and Gregori (2014); Piria et al. (2016b); Piria et al. (2018)

Remarks: non-native species; individual specimens reported in the Drava River

\section{Coregonus peled (Gmelin, 1789)}

Names under which the taxon was mentioned: Coregonus oxyrinchus (Linnaeus, 1758)

English name: Northern whitefish

Croatian name: sjeverna ozimica

Range in Croatia: Adriatic Basin - introduced to reservoirs on the Cetina River

Literature: Plančić (1938); Habeković (1978a); Vuković and Kosorić (1978); Habeković et al. (1981); Bojčić et al. (1982); Habeković (1982); Vrenk (1984); Treer (1985); Habeković and Čolo-Ančić (1986); Kažić et al. (1990); Jevtić (1991); Habeković (1993); Teskeredžić et al. (1993); Habeković (1994a); Crivelli (1995); Kerovec (1998); Schneider (1998); Mrakovčić et al. (2006); Tvrtković et al. (2009); Mrakovčić and Ćaleta (2011); Piria et al. (2016b); Piria et al. (2018)

Remarks: non-native species 
HUCHO Günther, 1866

93. Hucho hucho (Linnaeus, 1758)

Names under which the taxon was mentioned: Salmo hucho Linnaeus, 1758; Salvenius hucho; Epitomynis hucho Schulze, 1890

English name: Huchen

Croatian name: mladica

Range in Croatia: Danube drainage - upper course of the Kupa, Sava, Drava and Una Rivers

Literature: Seljan (1843); Bonaparte (1846); Jurinac (1880); Sebišanović (1880); Jurinac (1884); Glowacki (1885); Anonymous (1886); Kesterčanek (1886); Seeley (1886); Šoštarić (1888); Hefele (1889); Sebišanović (1889a); Sebišanović (1890); Brusina (1892); Franke (1892); Anonymous (1893a); Brusina (1893); Dudan (1893); Kišpatić (1893); Flögel (1894); Hawlitschek (1895); Glowacki (1896); Hirc (1896); Hirc (1897); Mojsisovics (1897); Hirc (1898); Steuer (1899); Anonymous (1900b); Horvat (1900d); Horvat (1901); Medić (1901b); Hirc (1902c); Anonymous (1903b); Langhoffer (1904); Brusina (1907); Anonymous (1909b); Ćurčić (1910); Vutskits (1918); Karaman (1923b); Plančić (1923a); Anonymous (1925); Bergleitner (1925); Kaitner (1926c); Anonymous (1927d); Anonymous (1927i); Bajić (1927); Anonymous (1928d); Bergleitner (1928); Anonymous (1930a); Anonymous (1930b); Anonymous (1930f); Rössler (1930); Anonymous (1931c); Kraus (1932); Mršić (1932a); Thaller (1932); Koritnik (1934); Anonymous (1935g); Plančić (1935a); Taler (1935b); Anonymous (1936a); Anonymous (1936c); Maldini (1936b); Thaler (1936); Anonymous (1937a); Anonymous (1937b); Hirtz (1937); Maldini (1938c); Taler (1944); Taler (1945b); Taler (1945a); Anonymous (1946a); Hafner-Lahorski (1947c); Knop (1947c); Hafner-Lahorski (1948a); Taler (1948d); Anonymous (1949); Taler (1950c); Taler (1951b); Taler (1953a); Taler (1953g); Anonymous (1955c); Kosorić (1955); Smlatić (1961); Sabioncello (1967); Pažur (1968b); Pažur (1969a); Anonymous (1969a); Sabioncello et al. (1970); Vuković and Ivanović (1971); Pažur (1975); Klašterka (1979); Sofradžija and Hadžiselimović (1981); Bojčić et al. (1982); Pažur et al. (1982); Vrenk (1984); Schulz (1985); Tvrtković (1985); Lelek (1987); Skalin (1990); Pažur (1993); Teskeredžić et al. (1993); Mrakovčić et al. (1996b); Honsig-Erlenburg et al. (1997); Majer (1998); Povž et al. (1998); Mustafić (2001); Georgiev (2003); Mateš (2004); Jakirčević (2005); Pažur (2005); Mrakovčić et al. (2006); Sallai and Mrakovčić (2007); Duplić (2008); Mateš (2008); Sallai and Kontos (2008); Valić et al. (2008); Dumbović et al. (2009); Sofradžija (2009); Bučar et al. (2010); Buj (2010c); Perović and Tvrtković (2010); Popović (2010); Freyhof (2012); Jelić et al. (2012); Witkowski et al. (2013); Treer et al. (2014); Ćaleta et al. (2015); Piria et al. (2016b); Pofuk et al. (2017)

Remarks: endemic to the Danube drainage
ONCORHYNCHUS Suckley, 1860

UI7 Oncorhynchus kisutch (Walbaum, 1792)

English name: Coho Salmon

Croatian name: srebrni losos

Range in Croatia: Danube drainage - introduced to the Vitunjčica and Dobra Rivers; Adriatic Basin - introduced in cage farming in the Adriatic Sea

Literature: Teskeredžić and Edwards (1987); Teskeredžić et al. (1989); Mateš (2004); Piria et al. (2018)

Remarks: non-native species; no self-sustaining populations established

UI8 Oncorhynchus masou (Brevoort, 1856)

Names under which the taxon was mentioned: Oncorhynchus masou rhodurus Jordan \& McGregor, 1925

English name: Masu salmon

Croatian name: japanski losos

Range in Croatia: Danube drainage - introduced to the Gračani fishpond; Adriatic Basin - introduced to the Krka River estuary

Literature: Teskeredžić and Teskeredžić (1990); Skaramuca et al. (1997)

Remarks: non-native species; no self-sustaining populations established

\section{Oncorhynchus mykiss (Walbaum, 1792)}

Names under which the taxon was mentioned: Parasalmo gairdneri (Richardson 1836); Salmo gairdneri Richardson, 1836; Salmo giardneri irideus Gibbons, 1855; Salmo irideus Gibbons, 1855; Trutta iridea (Gibbons, 1855)

English name: Rainbow trout

Croatian name: kalifornijska pastrva

Range in Croatia: Danube drainage and Adriatic Basin (except Istria) - introduced to upper course of rivers

Literature: Anonymous (1893b); Kišpatić (1893); Anonymous (1894b); Anonymous (1894f); Anonymous (1896c); Anonymous (1902); Kaitner (1926b); Anonymous (1927g); Anonymous (1928a); Anonymous (1929c); Anonymous (1930e); Anonymous (1931b); Thaller (1932); Anonymous (1935j); Mršić (1935c); Mršić (1935d); Mršić (1935a); Plančić (1935b); Taler (1935b); Taler (1935a); Gridelli (1936); Mršić (1938); Plančić (1938); Taler (1938); Anonymous (1946b); Bergleitner (1946); Taler (1946b); Taler (1946c); Taler (1948a); Taler (1948e); Anonymous (1949); Taler (1950b); Taler (1950c); Taler (1951c); Plančić et al. (1953); Taler (1954b); Anonymous (1955a); Bogdanović (1958); Taler (1958); Pilicar (1959); Bogdanović (1961); Knežević (1963a); Knežević (1963b); Anonymous (1965a); Vraneš (1966); Sabioncello (1967); Anonymus (1968); Pažur (1969c); Pažur (1969a); Pažur (1969b); Pažur 
(1970); Sabioncello et al. (1970); MacCrimmon (1971); Vuković and Ivanović (1971); Štefanac (1973); Pažur (1975); Habeković (1978b); Klašterka (1979); Mikuska (1979); Hrženjak and Ehrlich (1981); Bojčić et al. (1982); Habeković (1982); Mikuska (1983a); Mikuska (1983b); Teskeredžić (1983); Bristol et al. (1984); Ocvirk (1984); Teskeredžić (1984); Vrenk (1984); Al-Sabti (1985); Leiner (1985); Treer (1985); Teskeredžić et al. (1986); Teskeredžić and Pfeifer (1986); Kuhar and Huber (1989); Malnar (1989); Teskeredžić et al. (1989); Fašaić et al. (1990); Kažić et al. (1990); Mrakovčić and Mišetić (1990a); Habeković (1993); Leiner and Povž (1993); Teskeredžić et al. (1993); Habeković (1994a); Mrakovčić et al. (1995); Maitland and Crivelli (1996); Mrakovčić et al. (1996b); Honsig-Erlenburg et al. (1997); Skaramuca et al. (1997); Bonacci et al. (1998); Kerovec (1998); Povž et al. (1998); Schneider (1998); Leiner (1999); Mustafić (2001); Zupančič and Bogutskaya (2002); Schöffmann (2003); Mateš (2004); Jakirčević (2005); Mustafić (2005); Pažur (2005); Suić (2005); Šprem et al. (2005b); Treer et al. (2005); Mrakovčić et al. (2006); Knežević (2007); Prpa et al. (2007); Sallai and Mrakovčić (2007); Snoj et al. (2007); Valić et al. (2007); Duplić (2008); Kapetanović et al. (2008); Muhamedagić et al. (2008); Sallai and Kontos (2008); Valić et al. (2008); Treer et al. (2009); Tvrtković et al. (2009); Bučar et al. (2010); Bukvić et al. (2010); Pavličević et al. (2010); Vardić Smrzlić (2010); Mrakovčić and Ćaleta (2011); Glamuzina et al. (2011); Labak (2011); Jelić et al. (2012); Marčić et al. (2012); Marr et al. (2013); Vardić et al. (2013); Duplić (2014); Povž and Gregori (2014); Tomljanović (2014); Vukosav et al. (2014 (Ozimec (2015); Stanković et al. (2015); Jelić et al. (2016); Piria et al. (2016b); Pofuk et al. (2017); Piria et al. (2018)

Remarks: non-native species

\section{SALMO Linnaeus, 1758}

? Salmo dentex (Heckel, 1852)

Names under which the taxon was mentioned: Salar dentex Heckel, 1852; Salmo trutta dentex (Heckel, 1852); Trutta dentex

English name: Dentex trout

Croatian name: zubatak

Range in Croatia: Adriatic Basin - Cetina and Neretva Rivers

Literature: Heckel (1852); Heckel and Kner (1858); Canestrini (1866); Brusina (1872); Canestrini (1874); Brusina (1878); Giglioli (1880); Steindachner (1882b); Faber (1883); Katurić (1883); Kolombatović (1886a); Kolombatović (1886b); Seeley (1886); Katurić (1887); Kolombatović (1888); Katurić (1896a); Katurić (1896c); Brusina (1907); Katurić 1907); Car (1911); Ćurčić (1915); Anonymous (1930e); Thaller (1932); Karaman (1932); Karaman (1936); Karaman (1938); Kovačević (1952); Taler (1953a); Jedlička (1954); Stepanek (1956); Pešić (1961); Vuković (1963); Karaman (1966); Stepanek (1966);
Sabioncello (1967); Behnke (1968); Vuković and Ivanović (1971); Klašterka (1979); Bojčić et al. (1982); Tvrtković (1985); Economidis and Bănărescu (1991); Teskeredžić et al. (1993); Mrakovčić et al. (1995); Crivelli (1996); Kottelat (1997); Leiner (1998b); Kerovec (1998); Tvrtković (1998); Tutman et al. (2002); Mateš (2004); Razpet (2004); Pažur (2005); Mrakovčić et al. (2006); Kotellat (2007); Kottelat and Freyhof (2007); Mateš (2008); Muhamedagić et al. (2008); Tutman et al. (2008a); Valić et al. (2008); Glamuzina (2009); Sofradžija (2009); Tvrtković et al. (2009); Delling (2010); Glamuzina et al. (2010a); Glamuzina et al. (2010b); Pavličević et al. (2010); Georgiev (2011); Glamuzina et al. (2011); Mrakovčić and Ćaleta (2011); Glamuzina et al. (2013); Bianco (2014a); Ćaleta et al. (2015)

Remarks: the taxonomic status of this species is uncertain; no genetically distinct unit that would confirm to $S$. dentex has been found, therefore the name may be a synonym or corresponds to a morphotype of S. farioides or S. trutta; reports for the Cetina River have not been confirmed and are likely the result of misidentification; endemic to the Adriatic Basin

\section{Salmo farioides Karaman, 1938}

Names under which the taxon was mentioned: Salmo fario Linnaeus, 1758; Salmo trutta Linnaeus, 1758; Salmo trutta farioides; Salmo zrmanjensis, Karaman 1938; Salmo visovacensis Taler, 1950a; Trutta fario (Linnaeus, 1758)

English name: Adriatic trout

Croatian name: primorska pastrva

Range in Croatia: Adriatic Basin - rivers and lakes

Literature: Grisogono (1780); Lanza (1842); Kolombatović (1893); Kolombatović (1895); Katurić (1896a); Kolombatović (1907); Ćurčić (1915); Heintz (1915); Karaman (1927); Karaman (1936); Karaman (1938); Morović (1948); Pušić (1948); Taler (1950a); Taler (1950c); Basioli (1952); Kovačević (1952); Taler (1953a); Jedlička (1954); Stojnić (1965); Anonymous (1967); Sabioncello (1967); Behnke (1968); Marko et al. (1968); Sabioncello et al. (1970); Vuković and Ivanović (1971); Bojčić et al. (1982); Klašterka (1979); Mrakovčić and Mišetić (1990a); Leiner and Povž (1993); Teskeredžić et al. (1993); Mrakovčić et al. (1995); Mrakovčić et al. (1996a); Kottelat (1997); Leiner (1998b); Schneider (1998); Habeković and Mišetić (2000); Mikavica et al. (2001); Georgiev (2003); Schöffmann (2003); Mateš (2004); Razpet (2004); Mrakovčić et al. (2006); Vardić (2006); Kotellat (2007); Kottelat and Freyhof (2007); Kapetanović et al. (2008); Valić et al. (2008); Zupančič (2008); Glamuzina (2009); Bukvić et al. (2010); Glamuzina et al. (2010a); Glamuzina et al. (2010b); Pavličević et al. (2010); Georgiev (2011); Glamuzina et al. (2011); Glamuzina et al. (2013); MiočićStošić and Kovačević (2013); Bianco (2014a); Bianco (2014b); Ćaleta et al. (2015)

Remarks: Due to the unresolved taxonomy of the genus 
Salmo, it is not clear whether this is a distinct species or whether this name corresponds to the Adriatic lineage of $S$. trutta. Based on the most recent reports, the authors find $S$. farioides to be a valid name for reproductively isolated species.

\section{Salmo labrax Pallas, 1814}

\section{Names under which the taxon was mentioned:}

English name: Black Sea salmon

Croatian name: dunavska pastrva

Range in Croatia: Danube drainage - upper course of rivers

Literature: Holčík and Delić (2000); Delić et al. (2003a); Kottelat and Freyhof (2007); Bučar et al. (2010)

Remarks: Taxonomic status of this species is uncertain, although it is recognized as a separate taxonomic unit by majority of authors. However, the same unit has been described both as a separate species, S. labrax (e.g. Kottelat \& Freyhof, 2007) and as genetically distinct lineage inside $S$. trutta, named Danubian lineage (e.g. Bernatchez 2001, Bernatchez et al. 1992). Comprehensive taxonomic investigation is required to resolve taxonomic position of trout populations in Danube drainage in Croatia and elsewhere.

\section{Salmo marmoratus Cuvier, 1829}

Names under which the taxon was mentioned: Salar genivittatus marmorata Siebold, 1863; Salmo trotta, Linnaeus, 1758; Trutta genivittata (Heckel-Kner 1858); Trutta adriatica Kolombatović, 1890

English name: Marble trout

Croatian name: glavatica

Range in Croatia: Adriatic Basin - Neretva River drainage

Literature: Grisogono (1780); Lanza (1842); Heckel and Kner (1858); Kolombatović (1886a); Kolombatović (1886b); Kolombatović 1890); Kosić (1891); Kolombatović (1894); Katurić (1896a); Katurić (1896f); Kosić (1898); Kolombatović (1907); Brusina (1908); Ćurčić (1915); Heintz (1915); Jedlička (1926a); Jedlička (1926b); Taler (1926); Karaman (1927); Jedlička (1928); Anonymous (1929e); Thaller (1929); Anonymous (1930c); Anonymous (1930g); Anonymous (1930d); Anonymous (1930e); Ninni (1930); Karaman (1936); Karaman (1938); Ćurčić (1939d); Taler (1945b); Morović (1948); Pušić (1948); Taler (1950c); Basioli (1952); Kovačević (1952); Taler (1953a); Jedlička (1954); Stepanek (1956); Pešić (1961); Stojnić (1965); Stepanek (1966); Anonymous (1967); Sabioncello (1967); Behnke (1968); Marko et al. (1968); Kosorić (1969); Sabioncello et al. (1970); Vuković and Ivanović (1971); Klašterka (1979); Bojčić et al. (1982); Al-Sabti (1985); Mrakovčić and Mišetić (1990a); Leiner and Povž (1993); Teskeredžić et al. (1993); Bianco (1995a); Crivelli (1995);
Mrakovčić et al. (1995); Jardas (1996); Mrakovčić et al. (1996a); Kottelat (1997); Leiner (1998b); Mrakovčić (1998); Schneider (1998); Tvrtković (1998); Habeković and Mišetić (2000); Mikavica et al. (2001); Georgiev (2003); Schöffmann (2003); Mateš (2004); Razpet (2004); Pažur (2005); Mrakovčić et al. (2006); Vardić (2006); Kotellat (2007); Kottelat and Freyhof (2007); Kapetanović et al. (2008); Valić et al. (2008); Glamuzina (2009); Bukvić et al. (2010); Glamuzina et al. (2010a); Glamuzina et al. (2010b); Pavličević et al. (2010); Glamuzina et al. (2011); Glamuzina et al. (2013); Miočić-Stošić and Kovačević (2013); Bianco (2014a); Ćaleta et al. (2015); Piria et al. (2016b)

Remarks: Presence of this species in the Adriatic basin in Croatia is still uncertain, since no previous phylogenetic research was able to detect this species, even using specimens morphologically identified as S. marmoratus.

\section{Salmo obtusirostris (Heckel, 1852)}

Names under which the taxon was mentioned: Obtusirostris salonitana; Salmothyms obtusirostris (Heckel, 1852); Salmothymus obtusirostris krkensis (Karaman, 1927); Salmothymus obtusirostris obtusirostris; Salmothymus obtusirostris oxyrhynchus (Steindachner, 1882); Salmothymus obtusirostris salonitana (Karaman, 1927); Thymallus microlepis Steindachner, 1874; Trotta carpionata; Trutta obtusirostris; Trutta obtusirostris krkensis Karaman, 1927; Trutta obtusirostris oxyrhynchus Steindachner, 1882; Trutta obtusirostris salonitana Karaman, 1927

English name: Softmouth trout

Croatian name: mekousna

Range in Croatia: Adriatic Basin - upper course of the Jadro, Krka, Vrljika and Neretva Rivers

Literature: Grisogono (1780); Heckel (1852); Heckel and Kner (1858); Canestrini (1866); Canestrini (1874); Steindachner (1874); Steindachner (1875); Brusina (1878); Brusina (1880); Giglioli (1880); Kolombatović (1881); Steindachner (1882b); Faber (1883); Katurić (1883); Kolombatović (1886a); Kolombatović (1886b); Seeley (1886); Kolombatović (1888); Kolombatović (1895); Katurić (1896a); Petermann (1899); Kosić (1903); Brusina (1907); Katurić 1907); Kolombatović (1907); Car (1911); Ćurčić (1915); Heintz (1915); Jedlička (1925); Karaman (1927); Jedlička (1928); Anonymous (1930g); Anonymous (1930e); Jelačin (1931); Rössler (1931); Karaman (1932); Mršić (1932b); Thaller (1932); Mršić (1935b); Mršić (1935c); Karaman (1938); Mršić (1938); Ćurčić (1939b (Ćurčić (1939c); Morović (1948); Taler (1950c); Taler (1951a); Taler (1951b); Kovačević (1952); Taler (1952b); Taler (1952c); Uzelac (1952b); Anonymous (1953a); Ćurčić (1953); Škovrlj (1953a); Škovrlj (1953b); Taler (1953a); Taler (1953f); Jedlička (1954); Taler (1954a); Taler (1954c); Taler (1954d); Stepanek (1956); Basioli (1958b); Stevanović (1960); Pešić (1961); Stevanović 
(1961); Vuković (1963); Karaman (1966); Sabioncello (1967); Behnke (1968); Pažur (1969a); Sabioncello et al. (1970); Čanković et al. (1971); Vuković and Ivanović (1971); Klašterka (1979); Sofradžija and Hadžiselimović (1981); Bojčić et al. (1982); Tvrtković (1985); Malnar (1989); Mrakovčić (1990); Mrakovčić and Kerovec (1990a); Mrakovčić and Mišetić (1990a); Mrakovčić and Mišetić (1990b); Povž et al. (1990); Schöffmann (1991); Schöffmann (2004); Teskeredžić et al. (1993); Mrakovčić et al. (1995); Crivelli (1996); Maitland and Crivelli (1996); Kottelat (1997); Bonacci et al. (1998); Leiner (1998b); Mrakovčić (1998); Schneider (1998); Tvrtković (1998); Habeković and Pažur (2000); Mikavica et al. (2001); Odak et al. (2002); Snoj et al. (2002); Georgiev (2003); Odak et al. (2003); Schöffmann (2003); Treer et al. (2003b); Mateš (2004); Odak (2004); Razpet (2004); Treer et al. (2004); Pažur (2005); Treer et al. (2005); Mrakovčić et al. (2006); Tvrtković and Veen (2006); Kottelat and Freyhof (2007); Prpa et al. (2007); Snoj et al. (2007); Sušnik et al. (2007); Tomljanović et al. (2007); Duplić (2008); Jelić et al. (2008); Muhamedagić et al. (2008); Snoj et al. (2008); Treer et al. (2008a); Treer et al. (2008b); Valić et al. (2008); Glamuzina (2009); Treer et al. (2009); Glamuzina et al. (2010a); Glamuzina et al. (2010b); Pavličević et al. (2010); Popović (2010); Glamuzina et al. (2011); Cigrovski Mustafić (2012); Freyhof (2012); Tomljanović et al. (2012a); Glamuzina et al. (2013); Duplić (2014); Esteve et al. (2014); Pustovrh (2014); Tomljanović (2014); Ćaleta et al. (2015); Mustafić and Mrakovčić (2017); Nejedli et al. (2018); Piria et al. (2018)

Remarks: reports for the Zrmanja and Cetina Rivers, and Vrgorac have not been confirmed and are likely the result of misidentification; endemic to the Adriatic Basin

\section{UI9 Salmo salar Linnaeus, 1758}

English name: Atlantic salmon

Croatian name: atlantski losos

Range in Croatia: Danube drainage - one specimen from the Sava River; Adriatic Basin - mouth of the Neretva and Krka Rivers

Literature: Lanza (1842); Kažić et al. (1990); Piria et al. (2018)

Remarks: non-native species; it is not known whether these are individually introduced individuals or the result of misidentification; no self-sustaining populations established

\section{Salmo trutta Linnaeus, 1758}

Names under which the taxon was mentioned: Salar ausonii Valenciennes, 1848; Salmo fario Linnaeus, 1758; Salmo labrax Pallas, 1814; Salmo lacustris Linnaeus, 1758; Trutta fario (Linnaeus, 1758); Trutta likana Karaman, 1933

English name: Brown trout

\section{Croatian name: potočna pastrva}

Range in Croatia: Danube drainage - upper course of rivers; Adriatic Basin - rivers and lakes

Literature: Taube (1777); Piller and Mitterpacher (1783); Hietzinger (1817); Seljan (1843); Schlosser (1852); Heckel and Kner (1858); Farkaš Vukotinović (1859); Steindachner (1866a); Steindachner (1866b); Šloser (1870); Brusina (1878); Jurinac (1880); Borne (1881); Kolombatović (1881); Steindachner (1882b); Faber (1883); Jurinac (1884); Kolombatović (1884); Glowacki (1885); Kesterčanek (1886); Kolombatović (1888); Zlatović (1890); Hirc (1891); Kosić (1891); Anonymous (1892); Brusina (1892); Franke (1892); Anonymous (1893b); Anonymous (1893c); Brusina (1893); Dudan (1893); Kišpatić (1893); Anonymous (1894b); Anonymous (1894f); Flögel (1894); Anonymous (1896b); Anonymous (1896c); Buchwald (1896); Glowacki (1896); Hirc (1896); Anonymous (1897b); Anonymous (1897c); Dvoržak (1897); Hirc (1897); Franić (1898); Hirc (1898); Anonymous (1899a); Drnić (1899); Hirc (1899b); Hirc (1899c); Hirc (1899e); Franić (1900); Hirc (1900a); Hirc (1900b); Horvat (1900b); Bönel (1901); Talpaš (1901); Anonymous (1903a); Langhoffer (1904); Anonymous (1905c); Trgovčević (1905b); Anonymous (1906a); Anonymous (1906c); Anonymous (1907c); Brusina (1907); Brusina (1908); RHZZM (1908); Trgovčević (1908); Anonymous (1909a); Ribić (1909); Franić (1910); Kempf (1910); Hirc (1911); Lang (1911); Anonymous (1925); Bergleitner (1925); Hirc (1925); Jedlička (1925); Telar (1925); Grloci (1926); Anonymous (1927c); Anonymous (1927f); Anonymous (1928a); Anonymous (1928d); Anonymous (1928f); Anonymous (1928g); Kaitner (1928); Wettstein (1928); Anonymous (1929c); Anonymous (1929d); Bergleitner (1929); Kaitner (1929b); Rössler (1929a); Rössler (1929b); Anonymous (1930c); Anonymous (1930g); Anonymous (1930e); Bergleitner (1930); Jedlička (1930); Rössler (1930); Trgovčević (1930); Anonymous (1931c); Anonymous (1931d); Anonymous (1931f); Anonymous (1930g); Anonymous (1931h); Rössler (1931); Taler (1931); Karaman (1932); Krajačić (1932); Kraus (1932); Mršić (1932b); Mršić (1932a); Rössler (1932); Thaller (1932); Trgovčević (1932); Anonymous (1933); Koritnik (1934); Anonymous (1935a); Anonymous (1935b); Anonymous (1935c); Anonymous (1935f); Anonymous (1935h); Anonymous (1935i); Anonymous (1935j); Hafner-Lahorski (1935a); HafnerLahorski (1935b); Hafner-Lahorski (1935c); Mršić (1935b); Mršić (1935c); Mršić (1935a); Plančić (1935a); Plančić (1935b); Popović (1935); Taler (1935a); Taler (1935b); Maldini (1936b); Anonymous (1937b); Lovrić (1938); Maldini (1938c); Plančić (1938); Taler (1938); Anonymous (1939); Horvat (1941); Taler (1944); Anonymous (1945); Anonymous (1946b); Bergleitner (1946); Plančić (1946); Taler (1946c); Hafner-Lahorski (1947a); Hafner-Lahorski (1947b); Anonymous (1948a); Anonymous (1948b); Taler (1948a); Taler (1948c); Taler (1948d); Taler (1948e); Anonymous (1949); Glavan (1949); Taler (1949b); Plančić 
(1950); Taler (1950a); Taler (1950b); Taler (1950c); Taler (1951c); Taler (1951d); Taler (1951e); Taler (1951g); Anonymous (1952a); Anonymous (1952b); Knežević (1952); Plančić (1952c); Taler (1952a); Taler (1952d); Ćurčić (1953); Hafner-Lahorski (1953a); Plančić et al. (1953); Taler (1953a); Taler (1953b); Taler (1953e); Bogdanović (1954); Jedlička (1954); Kosorić (1955); Plančić (1956b); Plančić (1956c); Aganović (1957); Bogdanović (1957); Jagodić (1957); Anonymous (1958a); Bogdanović (1958); Kosorić (1958); Taler (1958); Bogdanović (1959); Stevanović (1960); Vuletić (1960); Bogdanović (1961); Smlatić (1961); Stevanović (1961); Knežević (1963b); Pažur (1964); Anonymous (1965b); Sabioncello (1967); Anonymus (1968); Behnke (1968); Pažur (1969c); Pažur (1969a); Pažur (1969b); Pažur (1970); Sabioncello et al. (1970); Vuković and Ivanović (1971); Štefanac (1973); Štefanac (1974); Pažur (1975); Aganović (1979); Klašterka (1979); Pažur (1979); Homen and Fašaić (1981); Hrženjak and Ehrlich (1981); Bojčić et al. (1982); Popović and Fašaić (1982); Štefanac and Bunjevčić (1982); Bristol et al. (1984); Delić (1984); Ocvirk (1984); Vrenk (1984); AlSabti (1985); Debeljak and Fašaić (1985); Leiner (1985); Tvrtković (1985); Štefanac (1986); Lelek (1987); Kuhar and Huber (1989); Mrakovčić et al. (1989); Fašaić et al. (1990); Habeković et al. (1992); Harka (1992); Mrakovčić (1992); Habeković (1993); Teskeredžić et al. (1993); Habeković (1994a); Kolar-Dimitrijević (1994); Crivelli (1995); Pažur (1995); Mrakovčić et al. (1996b); Habeković et al. (1997); Honsig-Erlenburg et al. (1997); Kottelat (1997); Skaramuca et al. (1997); Kerovec (1998); Majer (1998); Povž et al. (1998); Leiner (1999); Mustafić (2001); Trožić-Borovac (2002); Georgiev (2003); Jugović (2004); Mateš (2004); Odak (2004); Razpet (2004); Horvat (2005); Jakirčević (2005); Mustafić (2005); Pažur (2005); Suić (2005); Šprem et al. (2005b); Mrakovčić et al. (2006); Vardić (2006); Dulčić et al. (2007); Jadan et al. (2007); Knežević (2007); Kottelat and Freyhof (2007); Prpa et al. (2007); Sallai and Mrakovčić (2007); Sušnik et al. (2007); Valić et al. (2007); Jadan (2008); Jardas et al. (2008); Mateš (2008); Muhamedagić et al. (2008); Sallai and Kontos (2008); Treer et al. (2008a); Valić et al. (2008); Dumbović et al. (2009); Jelić et al. (2009); Treer et al. (2009); Tvrtković et al. (2009); Sofradžija (2009); Buj (2010c); Glamuzina et al. (2010a); Šafarek (2010); Valić (2010); Vardić Smrzlić (2010); Babajko (2011); Glamuzina et al. (2011); Mrakovčić and Ćaleta (2011); Randić (2011); Cigrovski Mustafić (2012); Jelić et al. (2012); Marčić et al. (2012); Marčić (2013); Marr et al. (2013); Muhamedagić and Habibović (2013); Vardić et al. (2013); Pustovrh (2014); Vukosav et al. (2014 (Jadan et al. (2015); Piria et al. (2016b); Piria et al. (2016c); Pofuk et al. (2017); Simonović et al. (2017a); Škraba et al. (2017); Barišić et al. (2018); Dragun et al. (2018); Piria et al. (2018); Mijošek et al. (2019)

Remarks: the genus Salmo is currently the subject of intensive study and the taxonomic position of individual populations is yet to be elucidated, given the early description of a large number of species in the Dinaric region of Croatia (S. visovacensis, S. zrmanjensis and $S$. likana). The taxonomic status of the species $S$. trutta and its origin in Croatia is not fully resolved. Some authors (e.g. Kottelat \& Freyhof, 2007) consider this species to be non-native in this part of Europe while recent papers (e. g. Schenekar et al. 2014) proved S. trutta to be native for some parts of the Danube basin. No self-sustaining populations have been established in Istria.

\section{SALVELINUS Richardson, 1836}

100. Salvelinus alpinus (Linnaeus, 1758)

\section{English name: Arctic charr}

Croatian name: jezerska zlatovčica

Range in Croatia: Danube drainage - introduced to the Plitvice Lakes; Adriatic Basin - Ruda River and reservoirs in the Cetina River drainage

Literature: Pažur (1970); Bristol et al. (1984); Habeković (1993); Kerovec (1998); Schneider (1998); Leiner (1999); Mateš (2004); Pažur (2005); Mrakovčić et al. (2006); Teskeredžić (2006); Tvrtković et al. (2009); Mrakovčić and Ćaleta (2011); Piria et al. (2016b); Pofuk et al. (2017); Piria et al. (2018)

Remarks: non-native species

UI10 Salvelinus fontinalis (Mitchill, 1814)

\section{English name: Brook trout}

Croatian name: potočna zlatovčica

Literature: Pešić (1961); Majer (1998); Mrakovčić et al. (2006); Vardić et al. (2007); Sallai (2008); Sallai and Kontos (2008); Jelić et al. (2012); Marr et al. (2013); Piria et al. (2016b); Pofuk et al. (2017); Piria et al. (2018)

Remarks: non-native species, no self-sustaining populations established. Not certain that it inhabits Croatian waters.

THYMALLUS Cuvier, 1829

101. Thymallus thymallus (Linnaeus, 1758)

Names under which the taxon was mentioned: Thymallus vexillifer Fitzinger, 1832; Thymallus vexillifer Perty, 1832; Thymallus vexillifer Agassiz, 1839-40; Thymallus vulgaris Nilsson, 1832

\section{English name: Grayling}

Croatian name: lipljen

Range in Croatia: Danube drainage - upper course of the Una and Kupa River systems; Adriatic Basin - introduced to the Gacka, Cetina, Ruda and Neretva Rivers

Literature: Canestrini (1866); Steindachner (1866b); Canestrini (1874); Sebišanović (1880); Borne (1881); Faber (1883); Anonymous (1886); Kesterčanek (1886); Seeley 
(1886); Mojsisovics (1889); Franke (1892); Brusina (1893); Dudan (1893); Kišpatić (1893); Glowacki (1896); Hirc (1896); Hirc (1897); Hirc (1898); Hirc (1898); Hirc (1899a); Hirc (1900a); Horvat (1900d); Medić (1901b); Anonymous (1904); Langhoffer (1904); Brusina (1907); RHZZM (1908); Vutskits (1918); Anonymous (1925); Bergleitner (1925); Anonymous (1927a); Anonymous (1928d); Bergleitner (1928); Anonymous (1930); Rössler (1930); Berg (1932a); Mršić (1932a); Koritnik (1934); Taler (1935b); Gridelli (1936); Maldini (1936b); Anonymous (1937b); Lovrić (1938); Bergleitner (1940); Taler (1944); Taler (1945b); Taler (1945a); Taler (1951b); Plančić (1952b); Taler (1953a); Kosorić (1955); Jagodić (1957); Sabioncello (1962); Smlatić (1961); Sabioncello (1967); Pažur (1969a); Sabioncello et al. (1970); Vuković and Ivanović (1971); Koritnik (1973); Štefanac (1973); Klašterka (1979); Sofradžija and Hadžiselimović (1981); Bojčić et al. (1982); Ocvirk (1984); Vrenk (1984); Leiner (1985); Tvrtković (1985); Lelek (1987); Habeković (1993); Teskeredžić et al. (1993); Habeković (1994a); Crivelli (1995); Mrakovčić et al. (1995); Mrakovčić et al. (1996b); Honsig-Erlenburg et al. (1997); Kerovec (1998); Majer (1998); Povž et al. (1998); Schneider (1998); Habeković and Mišetić (2000); Mikavica et al. (2001); Mustafić (2001); Delić et al. (2003a); Georgiev (2003); Jakirčević (2005); Mustafić (2005); Pažur (2005); Šprem et al. (2005a); Šprem et al. (2005b); Mrakovčić et al. (2006); Kottelat and Freyhof (2007); Prpa et al. (2007); Sallai and Mrakovčić (2007); Mateš (2008); Muhamedagić et al. (2008); Sallai and Kontos (2008); Treer et al. (2008a); Valić et al. (2008); Treer et al. (2009); Tvrtković et al. (2009); Sofradžija (2009); Bučar et al. (2010); Mrakovčić and Ćaleta (2011); Piria et al. (2016b); Pofuk et al. (2017); ); Nedić et al. (2018); Piria et al. (2018)

Remarks: non-native in the Adriatic Basin, in Istria there are no self-sustaining populations

\subsection{ORDER: GADIFORMES}

\subsubsection{FAMILY: LOTIDAE Bonaparte, 1835}

LOTA Oken, 1817

102. Lota lota (Linnaeus, 1758)

Names under which the taxon was mentioned: Lota vulgaris Fitzinger, 1832

English name: Burbot

Croatian name: manjić

Range in Croatia: Danube drainage - middle and lower course of rivers

Literature: Heckel and Kner (1858); Jurinac (1880); Sebišanović (1880); Sebišanović (1881); Mojsisovics (1883); Jurinac (1884); Mojsisovics (1884); Glowacki (1885); Anonymous (1886); Kesterčanek (1886); Jurinac (1887); Hefele (1889); Sebišanović (1890); Brusina (1892); Brusina (1893); Dudan (1893); Kišpatić (1893); Glowacki (1896); Hirc (1896); Medić (1896); Hirc (1897); Medić (1901b); RHZZM (1908); Ćurčić (1910); Kempf (1910); Lang (1911); Vutskits (1918); Plančić (1920); Plančić (1923a); Anonymous (1931c); Ćurčić (1933); Maldini (1936a); Pešić (1955); Sabioncello (1967); Vuković and Ivanović (1971); Klašterka (1979); Mikuska (1979); Bojčić et al. (1982); Veljović (1982); Mikuska (1983a); Mikuska (1983b); Tvrtković (1985); Veljović (1985); Habeković et al. (1986); Bojčić (1987); Lelek (1987); Mrakovčić (1992); Delić (1993); Mrakovčić et al. (1996b); Mustafić (2001); Mrakovčić et al. (2002); Delić et al. (2003a); Jugović (2004); Jakirčević (2005); Mustafić (2005); Pažur (2005); Sallai and Kontos (2005); Mrakovčić et al. (2006); Ćaleta (2007); Kottelat and Freyhof (2007); Sallai and Mrakovčić (2007); Mateš (2008); Sallai and Kontos (2008); Sevcsik and Erös (2008); Delić et al. (2009); Dumbović et al. (2009); Sofradžija (2009); Bučar et al. (2010); Buj (2010c); Jelkić et al. (2010); Ozimec et al. (2010); Jelić et al. (2012); Šanda et al. (2013); Zrnčić et al. (2013); Delić et al. (2014a); Piria et al. (2016a); Simonović et al. (2017b)

\subsection{ORDER: GOBIIFORMES}

\section{FAMILY: ODONTOBUTIDAE Hoese \& Gill, 1993}

\section{PERCCOTTUS Dybowski, 1877}

UI11 Perccottus glenii Dybowski, 1877

English name: Rotan

Croatian name: rotan

Range in Croatia: Danube drainage - channel of the Sava River near Slavonski Brod

Literature: Ćaleta et al. (2011b); Piria et al. (2016b); Piria et al. (2018)

Remarks: non-native, invasive species; one recorded individual, no self-sustaining populations established

\subsubsection{FAMILY: GOBIIDAE Cuvier, 1816}

BABKA Iljin, 1927

103. Babka gymnotrachelus (Kessler, 1857)

Names under which the taxon was mentioned: Gobius gymnotrahelus Kessler, 1857; Neogobius gymnotrachelus (Kessler, 1857)

English name: Racer goby

Croatian name: glavočić trkač

Range in Croatia: Danube drainage

Literature: Mojsisovics (1887); Polačik et al. (2008); Mustafić (2013); Šanda et al. (2013); Piria et al. (2016b); Nedić et al. (2018)

Remarks: non-native, invasive species 
KNIPOWITSCHIA Iljin, 1927

104. Knipowitschia mrakovcici Miller, 2009

Names under which the taxon was mentioned: Knipowitschia panizzae (Verga, 1841); Knipowitschia sp.

English name: Visovac goby

Croatian name: visovački glavočić

Range in Croatia: Adriatic Basin - Krka River drainage

Literature: Grisogono (1780); Miller (1990); Mrakovčić and Mišetić (1990a); Mrakovčić et al. (1995); Crivelli (1996); Maitland and Crivelli (1996); Mrakovčić et al. (2006); Valić (2006); Kottelat and Freyhof (2007); Duplić (2008); Jelić et al. (2008); Miller (2009); Freyhof (2011); Freyhof (2012); Vanhove et al. (2012); Ćaleta et al. (2015); Thacker et al. (2019)

Remarks: stenoendemic to the Krka River drainage and endemic to the Adriatic Basin and Croatia, species status is questioned in some recent papers

\section{Knipowitschia panizzae (Verga, 1841)}

Names under which the taxon was mentioned: Padogobius pannizai (Verga, 1841)

English name: Adriatic dwarf goby

Croatian name: glavočić vodenjak

Range in Croatia: Adriatic Basin - river mouths along the coast

Literature: Morović (1962); Sabioncello et al. (1964); Habeković (1967); Vuković and Ivanović (1971); Bojčić et al. (1982); Povž et al. (1990); Jardas (1996); Leiner (1998b); Kovačić and Pallaoro (2003); Miller (2004d); Zerunian (2004); Matić-Skoko et al. (2005); Mustafić (2005); Dulčić et al. (2007); Kottelat and Freyhof (2007); Kovačić (2008); Tvrtković et al. (2009); Sofradžija (2009); Tvrtković et al. (2009); Šanda and Kovačić (2009); Lipej and Dulčić (2010); Glamuzina et al. (2013); Ćaleta et al. (2015); Piria et al. (2016b); Vukić et al. (2016); Mustafić et al. (2017); Vukić et al. (2017); Thacker et al. (2019)

Remarks: endemic to the Adriatic Basin and the Adriatic Sea, reports of $K$. caucasica for Adriatic basin are result of misidentification and belong to $K$. panizzae

106. Knipowitschia radovici Kovačić, 2005

English name: Norin goby

Croatian name: norinski glavočić

Range in Croatia: Adriatic Basin - lower part of the Neretva River drainage

Literature: Kovačić (2005a); Mrakovčić et al. (2006); Kottelat and Freyhof (2007); Duplić (2008); Jelić et al. (2008); Glamuzina et al. (2010b); Freyhof (2011); Freyhof (2012); Vanhove et al. (2012); Glamuzina et al. (2013); Ćaleta et al. (2015); Thacker et al. (2019)
Remarks: endemic to the Neretva River drainage, endemic to the Adriatic Basin, species status is questioned in some recent papers

NEOGOBIUS Iljin, 1927

107. Neogobius fluviatilis (Pallas, 1814)

Names under which the taxon was mentioned: Gobius fluviatilis Pallas, 1814

English name: Monkey goby

Croatian name: riječni glavočić

Range in Croatia: Danube drainage - introduced to the Drava, Danube and Sava Rivers and their tributaries drainage

Literature: Vuković and Ivanović (1971); Lelek (1987); Mrakovčić et al. (2002); Mustafić (2005); Mrakovčić et al. (2006); Ćaleta (2007); Sallai and Mrakovčić (2007); Polačik et al. (2008); Sallai and Kontos (2008); Dumbović et al. (2009); Sofradžija (2009); Piria et al. (2011b); Jelić et al. (2012); Djikanović et al. (2013); Nedić et al. (2014a); Nedić et al. (2014b); Delić et al. (2014a); Riđanović et al. (2014); Jakšić (2016); Jakšić et al. (2016); Jakovlić et al. (2015); Piria et al. (2016a); Piria et al. (2016b); Simonović et al. (2017b); Piria et al. (2018)

Remarks: non-native, invasive species

108. Neogobius melanostomus (Pallas, 1814)

Names under which the taxon was mentioned: Gobius melanostomus Pallas, 1814

English name: Round goby

Croatian name: glavočić okrugljak

Range in Croatia: Danube drainage - introduced to the Drava, Danube and Sava Rivers and their tributaries

Literature: Mojsisovics (1887); Mustafić (2005); Mrakovčić et al. (2006); Kottelat and Freyhof (2007); Polačik et al. (2008); Piria et al. (2011c); Šanda et al. (2013); Jakšić (2016); Jakšić et al. (2016); Jakovlić et al. (2015); Piria et al. (2016a); Piria et al. (2016b); Simonović et al. (2017b)

Remarks: non-native, invasive species

NINNIGOBIUS Whitley, 1951

109. Ninnigobius canestrinii (Ninni, 1883)

Names under which the taxon was mentioned: Gobius canestrini Ninni, 1883; Pomatoschistus canestrinii (Ninni, 1883); Pomatoschistus marmoratus (Risso, 1810)

English name: Canestrini's goby

Croatian name: glavočić crnotrus

Range in Croatia: Adriatic Basin - river mouths along the coast

Literature: Ninni (1883); Kolombatović (1884); 
Kolombatović (1886a); Kolombatović (1888); Carus (18891893); Kolombatović (1891); Kolombatović (1907); RHZZM (1908); Taler (1953a); Sabioncello (1967); Vuković and Ivanović (1971); Bojčić et al. (1982); Jardas (1996); Holčík and Mrakovčić (1997); Kottelat (1997); Leiner (1998b); Nocita and Vanni (1997); Mrakovčić et al. (2000a); Miller (2004b); Zerunian (2004); Kovačić (2005b); Matić-Skoko et al. (2005); Mustafić (2005); Mrakovčić et al. (2006); Kottelat and Freyhof (2007); Jelić et al. (2008); Kovačić (2008); Miller and Šanda (2008); Tvrtković et al. (2009); Sofradžija (2009); Šanda and Kovačić (2009); Tutman et al. (2010); Freyhof (2011); Mrakovčić and Ćaleta (2011); Vanhove et al. (2012); Glamuzina et al. (2013); Bianco (2014a); Bianco (2014b); Ćaleta et al. (2015); Jelić et al. (2016); Piria et al. (2016b)

Remarks: endemic to the Adriatic Basin and to the Adriatic Sea

ORSINIGOBIUS Gandolfi, Marconato \& Torricelli, 1986

110. Orsinigobius croaticus Mrakovčić, Kerovec, Mišetić \& Schneider, 1994

Names under which the taxon was mentioned: Gobius punctatissimus Canestrini, 1864; Knipowitschia punctatissima (Canestrini, 1864); Knipowitschia punctatissima croatica Mrakovcic, Kerovec, Misetic \& Schneider, 1996; Knipowitschia croatica Mrakovcic, Kerovec, Misetic \& Schneider, 1996; Padogobius panizzai (Verga, 1841)

English name: Neretva dwarf goby

Croatian name: vrgoračka gobica

Range in Croatia: Adriatic Basin - Neretva River, Baćinska Lakes and Matica River near Vrgorac

Literature: Kolombatović (1895); Kolombatović (1907); Taler (1953a); Mrakovčić et al. (1994a); Mrakovčić et al. (1994b); Crivelli (1996); Mrakovčić et al. (1996a); Holčík and Mrakovčić (1997); Leiner (1998b); Schneider (1998); Tvrtković (1998); Mrakovčić et al. (2000a); Tutman et al. (2002); Trrtković and Franičević (2002); Miller (2004c); Miller (2004e); Zerunian (2004); Mrakovčić et al. (2006); Kottelat and Freyhof (2007); Duplić (2008); Jelić et al. (2008); Valić et al. (2008); Šanda and Kovačić (2009); Popović (2010); Mrakovčić and Ćaleta (2011); Zanella et al. (2011); Freyhof (2012); Vanhove et al. (2012); Glamuzina et al. (2013); Ćaleta et al. (2015); Mustafić and Mrakovčić (2017); Thacker et al. (2019)

Remarks: endemic to the Neretva River drainage and to the Adriatic Basin

\section{PADOGOBIUS Berg, 1932}

111. Padogobius bonelli (Bonaparte, 1846)

Names under which the taxon was mentioned: Padogobius martensii (Günther, 1861); Padogobius panizzae Berg, 1931
English name: Padanian goby

Croatian name: slatkovodni glavočić

Range in Croatia: Adriatic Basin - Mirna, Raša and Zrmanja River drainages, Vransko Lake near Biograd, introduced to the Ričica River in the Lika region

Literature: Anonymous (1967); Leiner (1985); Bianco and Miller (1990); Leiner and Povž (1993); Leiner (1994); Leiner and Povž (1994); Leiner et al. (1995); Mrakovčić et al. (1995); Crivelli (1996); Mrakovčić et al. (1996a); Schneider (1998); Mrakovčić et al. (2000b); Miller (2004f); Zerunian (2004); Mrakovčić et al. (2006); Kottelat and Freyhof (2007); Šanda and Kovačić (2009); Freyhof (2011); Miočić-Stošić and Kovačević (2013); Bianco (2014a); Bianco (2014b); Ćaleta et al. (2015); Jelić et al. (2016); Piria et al. (2016b)

Remarks: non-native in the Ričica River system in the Lika region; reports for the Krka River have not been confirmed; endemic to the Adriatic Basin

PONTICOLA Iljin, 1927

112. Ponticola kessleri (Günther 1861)

Names under which the taxon was mentioned: Gobius kessleri Günther, 1861; Neogobius kessleri (Günther, 1861)

English name: Bighead goby

Croatian name: keslerov galvoćić

Range in Croatia: Danube drainage - introduced to Drava, Danube and Sava Rivers

Literature: Vuković and Ivanović (1971); Mustafić (2005); Mrakovčić et al. (2006); Kottelat and Freyhof (2007); Polačik et al. (2008); Sofradžija (2009); Jelić et al. (2012); Šanda et al. (2013); Jakšić (2016); Jakšić et al. (2016); Jakovlić et al. (2015); Piria et al. (2016a); Piria et al. (2016b); Piria et al. (2018)

Remarks: non-native, invasive species

PROTERORHINUS Smitt, 1900

113. Proterorhinus semilunaris (Heckel, 1839)

Names under which the taxon was mentioned: Proterorhinus marmoratus (Pallas, 1814)

English name: Western tubenose goby

Croatian name: mramorasti glavočić

Range in Croatia: Danube drainage - Drava, Danube and Sava Rivers

Literature: Karaman (1952); Sabioncello (1967); Vuković and Ivanović (1971); Mikuska (1979); Mikuska (1983a); Mikuska (1983b); Mrakovčić et al. (1996b); Majer (1998); Miller (2004a); Mustafić (2005); Sallai and Kontos (2005); Mrakovčić et al. (2006); Sallai and Mrakovčić (2007); Sallai and Kontos (2008); Sofradžija (2009); Jelić et al. (2012); Piria et al. (2018) 


\subsection{ORDER: PLEURONECTIFORMES}

\subsubsection{FAMILY: PLEURONECTIDAE Rafinesque, 1815}

PLATICHTHYS Girard, 1856

114. Platichthys flesus Linnaeus, 1758

English name: European flounder

Croatian name: iverak

Range in Croatia: Adriatic Basin - river mouths, lagoons and lakes connected to the sea along the coast

Literature: Kosić (1903); Lorini (1921); Morović (1947); Morović (1948); Taler (1953a); Basioli (1957a); Basioli (1958a); Marčić Brusina (1963); Morović (1963); Morović (1976); Morović (1978); Bojčić et al. (1982); Jardas (1996); Leiner (1998b); Mikavica et al. (2001); Kottelat and Freyhof (2007); Jardas et al. (2008); Sofradžija (2009); Lipej and Dulčić (2010); Tutman et al. (2010); Glamuzina et al. (2013)

Remarks: euryhaline species that occasionally enters freshwater

\section{ORDER: CICHLIFORMES}

FAMILY: CICHLIDAE Bonaparte, 1835

OREOCHROMIS Günther, 1889

U112 Oreochromis niloticus (Linnaeus, 1758)

English name: Nile tilapia

Croatian name: nilska tilapia

Range in Croatia: Danube drainage - Lonjsko Field

Literature: Duplić and Boršić (2010); Povž and Gregori (2014); Piria et al. (2018)

Remarks: non-native species; individual reports without the establishment of self-sustaining populations

\subsection{ORDER: CYPRINODONTIFORMES}

\subsubsection{FAMILY: CYPRINODONTIDAE Wagner, 1828}

APHANIUS Nardo, 1827

115. Aphanius fasciatus (Valenciennes, 1821)

Names under which the taxon was mentioned: Cyprinodon calaritanus (Cuvier, 1829); Lebias calaritana (Cuvier, 1829)

English name: Mediterranean toothcarp

Croatian name: obrvan

Range in Croatia: Adriatic Basin - mostly saline habitats along the Adriatic coast

Literature: Bonaparte (1846); Canestrini (1866); Kolombatović (1886a); Kolombatović (1886b); Kolombatović (1893); Car (1900); Langhoffer (1904); Brusina (1907); Vuković and Ivanović (1971); Bojčić et al. (1982); Crivelli (1996); Jardas (1996); Pallaoro (1998);
Matić-Skoko et al. (2005); Dulčić and Glamuzina (2006); Mrakovčić et al. (2006); Kottelat and Freyhof (2007); Jardas et al. (2008); Lipej and Dulčić (2010); Glamuzina et al. (2013); Buj et al. (2015b); Landeka et al. (2015); Marčić et al. (2015); Piria et al. (2016b); Mustafić et al. (2017)

\subsubsection{FAMILY: POECILIIDAE Bonaparte, 1835}

GAMBUSIA Poey, 1854

116. Gambusia holbrooki Girard, 1859

Names under which the taxon was mentioned: Gambusia affinis (Baird \& Girard 1853); Gambusia affinis holbrooki (Girard, 1859)

English name: Eastern mosquitofish

Croatian name: gambuzija

Range in Croatia: Danube drainage - introduced to the Savica Lakes in the Sava River system in Zagreb; Adriatic Basin - introduced to rivers, lakes and ponds

Literature: Gioseffi (1926); Anonymous (1927e); Sella (1927); Sella (1928); Sella (1929); Anonymous (1934); Gridelli (1936); Dojmi (1938); Nežić (1938); Plančić (1948); Tutman (1948); Morović (1964a); Sabioncello et al. (1964); Anonymous (1965b); Habeković (1967); Sabioncello (1967); Morović (1970); Vuković and Ivanović (1971); Morović (1976); Bojčić et al. (1982); Leiner (1984); Treer et al. (1984); Leiner (1985); Tvrtković (1985); Treer (1989); Fašaić et al. (1990); Mrakovčić and Mišetić (1990a); Habeković (1993); Leiner and Povž (1993); Leiner and Povž (1994); Crivelli (1995); Leiner et al. (1995); Mrakovčić et al. (1995); Maitland and Crivelli (1996); Mrakovčić et al. (1996a); Holčík and Mrakovčić (1997); Bonacci et al. (1998); Kerovec (1998); Leiner (1998b); Schneider (1998); Mrakovčić et al. (2000a); Mikavica et al. (2001); Bakić (2006); Dulčić and Glamuzina (2006); Mrakovčić et al. (2006); Dulčić et al. (2007); Duplić (2008); Jakšić et al. (2008); Marguš (2008); Tvrtković et al. (2009); Bakić (2011); Mrakovčić and Ćaleta (2011); Treer et al. (2011); Glamuzina et al. (2013); Lucu and Marsoni (2013); Marr et al. (2013); Miočić-Stošić and Kovačević (2013); Radošević (2013); Landeka et al. (2015); Piria et al. (2016b); Mustafić et al. (2017); Pofuk et al. (2017); Piria et al. (2018)

Remarks: non-native species; reports of $G$. affinis in Istria have not been confirmed

\subsection{ORDER: MUGILIFORMES}

\subsubsection{FAMILY: MUGILIDAE Jarocki, 1822}

\section{CHELON Artedi, 1793}

117. Chelon labrosus (Risso, 1827)

Names under which the taxon was mentioned: Mugil chelo Cuvier, 1829; Crenimugil labrasus (Risso, 1827); Mugil labrosus Risso, 1827 
English name: Thicklip Grey Mullet

Croatian name: cipal putnik

Range in Croatia: Adriatic Basin - river mouths, lagoons and lakes connected to the sea along the coast

Literature: Kolombatović (1881); Kišpatić (1893); Katurić 1907); Šoljan (1931); Morović (1956a); Morović (1956b); Morović (1962); Morović (1963); Morović (1964b); Sabioncello et al. (1964); Habeković (1967); Sabioncello (1967); Morović (1976); Bojčić et al. (1982); Treer (1989); Jardas (1996); Leiner (1998b); Dulčić and Glamuzina (2006); Kottelat and Freyhof (2007); Sofradžija (2009); Glamuzina and Bartulović (2010); Lipej and Dulčić (2010); Tutman et al. (2010); Glamuzina et al. (2013)

Remarks: euryhaline species that occasionally enters freshwater

LIZA Jordan \& Swain, 1884

118. Liza aurata (Risso, 1810)

Names under which the taxon was mentioned: Mugil auratus Risso, 1810

English name: Golden Grey Mullet

Croatian name: cipal zlatac (zlatar)

Range in Croatia: Adriatic Basin - river mouths, lagoons and lakes connected to the sea along the coast

Literature: Kišpatić (1893); Morović (1948); Basioli (1952); Morović (1956a); Morović (1956b); Morović (1956c); Basioli (1957a); Basioli (1958a); Morović (1962); Morović (1963); Morović (1964a); Morović (1964b); Sabioncello et al. (1964); Habeković (1967); Morović (1967); Morović (1976); Sofradžija and Hadžiselimović (1981); Bojčić et al. (1982); Treer (1989); Jardas (1996); Kraljević and Dulčić (1996); Leiner (1998b); Mustafić (2005); Dulčić and Glamuzina (2006); Kottelat and Freyhof (2007); Valić et al. (2007); Sofradžija (2009); Tvrtković et al. (2009); Bartulović et al. (2010); Glamuzina and Bartulović (2010); Lipej and Dulčić (2010); Tutman et al. (2010); Mrakovčić and Ćaleta (2011); Šiljeg (2012); Glamuzina et al. (2013)

Remarks: euryhaline species that occasionally enters freshwater

\section{Liza ramada (Risso, 1827)}

Names under which the taxon was mentioned: Mugil capito Cuvier, 1829; Mugil ramada Risso, 1827

English name: Thin-lipped grey mullet

Croatian name: cipal balavac

Range in Croatia: Adriatic Basin - river mouths, lagoons and lakes connected to the sea along the coast

Literature: Carrara (1846); Kišpatić (1893); Car (1911); Morović (1956a); Morović (1956b); Morović (1962); Morović (1963); Morović (1964a); Morović (1964b); Sabioncello et al. (1964); Habeković (1967); Morović
(1967); Morović (1976); Bojčić et al. (1982); Bojčić (1987); Treer (1989); Jardas (1996); Leiner (1998b); Mrakovčić et al. (2000a); Dulčić and Glamuzina (2006); Bartulović et al. (2007); Kottelat and Freyhof (2007); Sofradžija (2009); Glamuzina and Bartulović (2010); Lipej and Dulčić (2010); Tutman et al. (2010); Šiljeg (2012); Glamuzina et al. (2013)

Remarks: euryhaline species that occasionally enters freshwater

\section{Liza saliens (Risso, 1810)}

Names under which the taxon was mentioned: Mugil saliens Risso, 1810

English name: Leaping mullet

Croatian name: cipal dugaš

Range in Croatia: Adriatic Basin - river mouths, lagoons and lakes connected to the sea along the coast

Literature: Carrara (1846); Kišpatić (1893); Morović (1948); Basioli (1952); Morović (1956a); Morović (1956b); Basioli (1957a); Basioli (1958a); Morović (1962); Morović (1963); Morović (1964b); Sabioncello et al. (1964); Habeković (1967); Morović (1976); Sofradžija and Hadžiselimović (1981); Bojčić et al. (1982); Treer (1989); Leiner (1998b); Jardas (1996); Dulčić and Glamuzina (2006); Kottelat and Freyhof (2007); Sofradžija (2009); Glamuzina and Bartulović (2010); Lipej and Dulčić (2010); Tutman et al. (2010); Glamuzina et al. (2013)

Remarks: euryhaline species that occasionally enters freshwater

MUGIL Linnaeus, 1758

121. Mugil cephalus Linnaeus, 1758

English name: Flathead mullet

Croatian name: cipal bataš (glavaš)

Range in Croatia: Adriatic Basin - river mouths, lagoons and lakes connected to the sea along the coast

Literature: Lanza (1842); Carrara (1846); Kišpatić (1893); Šoljan (1931); Morović (1947); Morović (1948); Fijan (1951a); Basioli (1952); Morović (1956a); Morović (1956b); Morović (1956c); Basioli (1957b); Basioli (1958a); Morović (1962); Morović (1963); Anonymous (1964); Morović (1964a); Morović (1964b); Sabioncello et al. (1964); Habeković (1967); Morović (1967); Morović (1976); Morović (1978); Sofradžija and Hadžiselimović (1981); Bojčić et al. (1982); Bojčić (1987); Treer (1989); Jardas (1996); Tvrtković (1998); Leiner (1998b); Mrakovčić et al. (2000a); Mikavica et al. (2001); Treer et al. (2003c); Dulčić and Glamuzina (2006); Kottelat and Freyhof (2007); Muhamedagić et al. (2008); Sofradžija (2009); Bartulović et al. (2010); Bukvić et al. (2010); Dulčić and Glamuzina (2010); Glamuzina (2010); Glamuzina and Bartulović (2010); Lipej and Dulčić (2010); Marević (2010); Tutman et al. (2010); Glamuzina et al. (2013) 
Remarks: euryhaline species that occasionally enters freshwater

OEDALECHILUS Fowler, 1903

122. Oedalechilus labeo Cuvier, 1829

Names under which the taxon was mentioned: Mugil labeo Cuvier, 1829

English name: Boxlip mullet

Croatian name: cipal plutaš

Range in Croatia: Adriatic Basin - river mouths, lagoons and lakes connected to the sea along the coast

Literature: Kosić (1899); Kišpatić (1893); Morović (1956a); Morović (1956a); Morović (1956b); Bojčić et al. (1982); Jardas (1996); Lipej and Dulčić (2010); Tutman et al. (2010)

Remarks: euryhaline species that occasionally enters freshwater

\subsection{ORDER: BLENNIIFORMES}

\subsubsection{FAMILY: BLENNIIDAE Rafinesque, 1810}

\section{SALARIA Forsskål, 1775}

123. Salaria fluviatilis (Asso y del Rio, 1801)

Names under which the taxon was mentioned: Blennius cagnota Valenciennes, 1836; Blennius fluviatilis Asso, 1801; Blennius pavo Risso, 1810; Blennius Petteri Heckel \& Kner, 1858; Blennius sanguinolentus Pallas, 1814; Blennius vulgaris Pollini, 1816; Lipophrys fluviatilis (Asso, 1801)

English name: Freshwater blenny

Croatian name: riječna babica

Range in Croatia: Adriatic Basin - Zrmanja, Krka, Cetina and Neretva River drainages, Vransko Lake near Biograd, Baćinska Lakes

Literature: Carrara (1846); Heckel and Kner (1858); Kolombatović (1886a); Kolombatović (1886b); Seeley (1886); Kolombatović (1888); Kolombatović (1892); Kišpatić (1893); Katurić 1907); Kolombatović (1907); Katurić (1908); Plančić (1952d); Taler (1953a); Basioli (1960); Morović (1962); Vuković (1963); Sabioncello et al. (1964); Sabioncello (1967); Morović (1970); Vuković and Ivanović (1971); Bojčić et al. (1982); Treer (1989); Fašaić et al. (1990); Mrakovčić and Mišetić (1990a); Habeković (1993); Mrakovčić et al. (1995); Crivelli (1996); Jardas (1996); Maitland and Crivelli (1996); Holčík and Mrakovčić (1997); Kottelat (1997); Kerovec (1998); Leiner (1998b); Pallaoro (1998); Schneider (1998); Mrakovčić et al. (2000a); Bath (2003); Mustafić (2005); Mrakovčić et al. (2006); Valić (2006); Kottelat and Freyhof (2007); Marguš (2008); Tvrtković et al. (2009); Sofradžija (2009); Glamuzina et al. (2010b); Mrakovčić and Ćaleta (2011); Glamuzina et al. (2013); Miočić-Stošić and Kovačević (2013); Jelić et al. (2016); Piria et al. (2016c); Mustafić et al. (2017)
Remarks: introduced to Ričica River (Lika region) from the Zrmanja River

\subsection{ORDER: SCORPAENIFORMES}

\subsubsection{FAMILY: COTTIDAE Bonaparte, 1831}

\section{COTTUS Linnaeus, 1758}

124. Cottus gobio Linnaeus, 1758

Names under which the taxon was mentioned: Cottus poecilopus Heckel, 1838; Cottus ferrugineus Bonaparte, 1846; Cottus ferrugineus Heckel \& Kner, 1858; Cottus gobio ferugineus

\section{English name: Bullhead}

\section{Croatian name: peš}

Range in Croatia: Danube drainage - upper course of tributaries; Adriatic Basin - Zrmanja, Krka and Neretva River drainages

Literature: Piller and Mitterpacher (1783); Carrara (1846); Heckel and Kner (1858); Steindachner (1866a); Brusina (1874); Brusina (1878); Jurinac (1880); Jurinac (1884); Glowacki (1885); Jurinac (1886a); Kolombatović (1886a); Seeley (1886); Mojsisovics (1887); Kolombatović (1888); Brusina (1889); Brusina (1892); Kišpatić (1893); Glowacki (1896); Hirc (1896); Hirc (1897); Hirc (1898); Hirc (1899c); Hirc (1900a); Langhoffer (1904); Brusina (1907); Katurić 1907); Kolombatović (1907); RHZZM (1908); Lang (1911); Vutskits (1918); Karaman (1923b); Anonymous (1925); Bergleitner (1925); Karaman (1928); Rössler (1930); Rössler (1931); Fink (1932b); Rössler (1932); Koritnik (1934); Maldini (1935c); Taler (1935a); Taler (1935b); Maldini (1938b); Taler (1953a); Kosorić (1955); Stepanek (1956); Smlatić (1961); Anonymous (1967); Sabioncello (1967); Sabioncello et al. (1969); Pažur (1970); Vuković and Ivanović (1971); Klašterka (1979); Sofradžija and Hadžiselimović (1981); Bojčić et al. (1982); Tvrtković (1985); Habeković et al. (1986); Lelek (1987); Mrakovčić (1992); Mrakovčić et al. (1995); Mrakovčić et al. (1996b); Honsig-Erlenburg et al. (1997); Kottelat (1997); Leiner (1998b); Leiner (1998a); Majer (1998); Povž et al. (1998); Schneider (1998); Mikavica et al. (2001); Mustafić (2001); Delić et al. (2003a); Šlechtová et al. (2004); Zerunian (2004); Freyhof et al. (2005b); Mustafić (2005); Sallai and Kontos (2005); Mrakovčić et al. (2006); Kottelat and Freyhof (2007); Prpa et al. (2007); Sallai and Mrakovčić (2007); Jelić et al. (2008); Muhamedagić et al. (2008); Sallai and Kontos (2008); Sevcsik and Erös (2008); Treer et al. (2008a); Dumbović et al. (2009); Jelić et al. (2009); Sofradžija (2009); Treer et al. (2009); Bučar et al. (2010); Buj (2010c); Jelkić et al. (2010); Perović and Tvrtković (2010); Babajko (2011); Ištok (2011); Jelić et al. (2012); Jelić (2012); Miočić-Stošić and Kovačević (2013); Bianco (2014a); Piria et al. (2016c)

Remarks: the population from the Zrmanje River was described as C. ferrugineus, though molecular studies 
(Šlechtová et al. 2004) have indicated its similarity to C. gobio, and therefore $C$. ferrugineus is not listed as a separate species.

\subsection{ORDER: PERCIFORMES}

\subsubsection{FAMILY: MORONIDAE Jordan \& Evermann, 1896}

\section{DICENATRARCHUS Gill, 1860}

\section{Dicentrarchus labrax Linnaeus, 1758}

English name: European sea bass

Croatian name: lubin, brancin

Range in Croatia: Adriatic Basin - river mouths, lagoons and lakes connected to the sea along the coast

Literature: Grisogono (1780); Lanza (1842); Anonymous (1906a); Lorini (1921); Šoljan (1931); Morović (1947); Morović (1948); Šoljan (1948); Basioli (1952); Kovačević (1952); Plančić (1952c); Taler (1953a); Basioli (1957a); Basioli (1958a); Grce (1959); Basioli (1960); Morović (1962); Marčić Brusina (1963); Morović (1963); Vuković (1963); Sabioncello et al. (1964); Habeković (1967); Vuković and Ivanović (1971); Morović (1976); Bojčić et al. (1982); Jardas (1996); Treer et al. (1989); Leiner (1998b); Schneider (1998); Kottelat and Freyhof (2007); Sofradžija (2009); Lipej and Dulčić (2010); Tutman et al. (2010); Glamuzina et al. (2013)

Remarks: euryhaline species that occasionally enters freshwater

MORONE Mitchill, 1814

\section{UI13 Morone saxatilis x Morone chrysops}

Hybrid of a striped bass (Morone saxatilis) female and a white bass (M. chrysops) male

English name: Palmetto bass

Croatian name: palmetto grgeč

Range in Croatia: Danube drainage - introduced to the Danube River

Literature: Safner et al. (2013); Piria et al. (2016b); Piria et al. (2018)

Remarks: non-native species; one individual recorded in the Danube River

\subsubsection{FAMILY: CENTRARCHIDAE Regan, 1913}

LEPOMIS Rafinesque, 1819

126. Lepomis gibbosus (Linnaeus, 1758)

Names under which the taxon was mentioned: Eupomotis gibbosus (Linnaeus, 1758)

English name: Pumpkinseed

Croatian name: sunčanica
Range in Croatia: Danube drainage and Adriatic Basin introduced to rivers, lakes and reservoirs

Literature: Kempf (1910); Plančić (1920); Anonymous (1925); Anonymous (1946a); Taler (1947); Taler (1951e); Karaman (1952); Taler (1953a); Plančić (1955); Grce (1956); Grce (1957); Grce (1959); Vuletić (1960); Livojević (1962a); Livojević (1962b); Morović (1962); Morović (1964a); Sabioncello et al. (1964); Morović (1967); Sabioncello (1967); Pažur (1968a); Zobundžija (1968); Vuković and Ivanović (1971); Čanadjija (1977); Klašterka (1979); Mikuska (1979); Bojčić et al. (1982); Veljović (1982); Mikuska (1983a); Mikuska (1983b); Mikuska and Mamić (1984); Ocvirk (1984); Mikuska (1985); Veljović (1985); Habeković et al. (1986); Delić (1989); Treer (1989); Habeković et al. (1990); Habeković and Popović (1991); Homen et al. (1991); Mrakovčić (1992); Delić (1993); Habeković (1993); Mrakovčić et al. (1995); Povž (1995); Zrnčić (1995); Mihaljević et al. (1996); Mrakovčić et al. (1996b); Habeković et al. (1997); Kerovec (1998); Leiner (1998a); Leiner (1998b); Majer (1998); Schneider (1998); Treer et al. (1998); Zanella et al. (2000); Jakovlić and Treer (2001); Mustafić (2001); Mrakovčić et al. (2002); Bakota et al. (2003); Mateš (2004); Jakirčević (2005); Mustafić (2005); Pažur (2005); Suić (2005); Mrakovčić et al. (2006); Ćaleta (2007); Knežević (2007); Kottelat and Freyhof (2007); Prpa et al. (2007); Sallai and Mrakovčić (2007); Duplić (2008); Marguš (2008); Sallai and Kontos (2008); Sevcsik and Erös (2008); Treer et al. (2008a); Delić et al. (2009); Dumbović et al. (2009); Jelić et al. (2009); Kerestessy et al. (2009); Sofradžija (2009); Treer et al. (2009); Bučar et al. (2010); Buj (2010c); Bukvić et al. (2010); Jelić et al. (2010); Ozimec et al. (2010); Vardić Smrzlić (2010); Mrakovčić and Ćaleta (2011); Jelić et al. (2012); Marčić et al. (2012); Glamuzina et al. (2013); Jelkić and Opačak (2013); Marr et al. (2013); Miočić-Stošić and Kovačević (2013); Delić et al. (2014a); Povž and Gregori (2014); Jelić et al. (2016); Piria et al. (2016a); Piria et al. (2016b); Jelić and Krivek (2017); Mustafić et al. (2017); Piria et al. (2017); Pofuk et al. (2017); Simonović et al. (2017b); Piria et al. (2018)

Remarks: non-native, invasive species

MICROPTERUS Lacepède, 1802

127. Micropterus salmoides (Lacepède 1802)

Names under which the taxon was mentioned: Grystes salmoides (Lacepède, 1802)

English name: Largemouth bass

Croatian name: pastrvski grgeč

Range in Croatia: Danube drainage - introduced to rivers, channels, ponds and backwaters; Adriatic Basin - Neretva River drainage

Literature: Anonymous (1907a); Hasenay (1933); Fijan (1950); Karaman (1952); Sabioncello (1967); Pogrmilović (1972); Tóth and Biro (1982); Bojčić (1987); Mrakovčić 
(1992); Mrakovčić et al. (1996b); Majer (1998); Mustafić (2001); Mrakovčić et al. (2002); Pažur (2005); Mrakovčić et al. (2006); Sallai and Mrakovčić (2007); Sallai and Kontos (2008); Ozimec et al. (2010); Jelić et al. (2012); Povž and Gregori (2014); Piria et al. (2016b); Dulčić et al. (2017); Piria et al. (2018)

Remarks: non-native, invasive species

\subsubsection{FAMILY: PERCIDAE Rafinesque, 1815}

GYMNOCEPHALUS Bloch, 1793

128. Gymnocephalus baloni Holcík \& Hensel, 1974

English name: Balon's ruffe

Croatian name: Balonov balavac

Range in Croatia: Danube drainage - Mura, Drava, Danube and Sava Rivers

Literature: Lelek (1987); Mrakovčić (1992); Mrakovčić et al. (1996b); Povž et al. (1997); Majer (1998); Mustafić (2001); Mustafić (2005); Sallai and Kontos (2005); Mrakovčić et al. (2006); Kottelat and Freyhof (2007); Sallai and Mrakovčić (2007); Sallai and Kontos (2008); Kerestessy et al. (2009); Popović (2010); Jelić et al. (2012)

\section{Gymnocephalus cernua (Linnaeus, 1758)}

Names under which the taxon was mentioned: Acerina rosica; Acerina cernua (Linnaeus, 1758); Acerina vulgaris Cuvier, 1829; Gynmocephalus cernuus (Linnaeus, 1758); Perca cernua Linnaeus, 1758

English name: Ruffe

Croatian name: obični balavac

Range in Croatia: Danube drainage - lower course of rivers; Adriatic Basin - introduced to the Lika River system

Literature: Heckel and Kner (1858); Jurinac (1880); Sebišanović (1880); Jurinac (1881); Sebišanović (1881); Mojsisovics (1883); Jurinac (1884); Mojsisovics (1884); Glowacki (1885); Anonymous (1886); Kesterčanek (1886); Mojsisovics (1887); Sebišanović (1889b); Sebišanović (1890); Brusina (1892); Brusina (1893); Dudan (1893); Kišpatić (1893); Glowacki (1896); Hirc (1897); Brusina (1898); Hirc (1899e); Langhoffer (1904); Anonymous (1906d); RHZZM (1908); Ćurčić (1910); Vutskits (1918); Uzelac (1952a); Sabioncello (1953); Sabioncello (1967); Zobundžija (1968); Sabioncello et al. (1969); Vuković and Ivanović (1971); Klašterka (1979); Mikuska (1979); Sofradžija and Hadžiselimović (1981); Bojčić et al. (1982); Veljović (1982); Mikuska (1983a); Mikuska (1983b); Mikuska and Mamić (1984); Mikuska (1985); Veljović (1985); Habeković et al. (1986); Lelek (1987); Delić (1989); Habeković et al. (1990); Homen et al. (1991); Mrakovčić (1992); Delić (1993); Mrakovčić et al. (1996b); Leiner (1998a); Majer (1998); Treer et al. (1998); Zanella et al. (2000); Jakovlić and Treer (2001); Mustafić (2001);
Mrakovčić et al. (2002); Jugović (2004); Jakirčević (2005); Mustafić (2005); Pažur (2005); Ćaleta (2007); Kottelat and Freyhof (2007); Prpa et al. (2007); Sallai and Mrakovčić (2007); Sallai and Kontos (2008); Treer et al. (2008a); Dumbović et al. (2009); Jelić et al. (2009); Kerestessy et al. (2009); Sofradžija (2009); Treer et al. (2009); Jelić et al. (2010); Ozimec et al. (2010); Jelić et al. (2012); Jelkić and Opačak (2013); Delić et al. (2014a); Piria et al. (2016a); Jelić et al. (2016b); Pofuk et al. (2017); Simonović et al. (2017b)

Remarks: non-native in the Adriatic Basin

130. Gymnocephalus schraetser (Linnaeus, 1758)

Names under which the taxon was mentioned: Acerina schraetser (Linnaeus, 1758)

English name: Striped ruffe

Croatian name: prugasti balavac

Range in Croatia: Danube drainage - Drava, Danube and Sava Rivers

Literature: Heckel and Kner (1858); Jurinac (1880); Sebišanović (1880); Sebišanović (1881); Jurinac (1884); Glowacki (1885); Seeley (1886); Mojsisovics (1887); Sebišanović (1890); Brusina (1893); Kišpatić (1893); Glowacki (1896); Hirc (1899e); Langhoffer (1904); Ćurčić (1910); Vutskits (1918); Karaman (1923b); Maldini (1935a); Sabioncello (1953); Sabioncello (1967); Vuković and Ivanović (1971); Klašterka (1979); Mikuska (1979); Bojčić et al. (1982); Habeković et al. (1986); Lelek (1987); Delić (1989); Homen et al. (1991); Mrakovčić (1992); Delić (1993); Mrakovčić et al. (1996b); Majer (1998); Mustafić (2001); Jakirčević (2005); Mustafić (2005); Pažur (2005); Sallai and Kontos (2005); Mrakovčić et al. (2006); Ćaleta (2007); Kottelat and Freyhof (2007); Sallai and Mrakovčić (2007); Sallai and Kontos (2008); Valić et al. (2008); Sofradžija (2009); Ozimec et al. (2010); Popović (2010); Jelić et al. (2012); Ćaleta et al. (2015); Piria et al. (2016b)

Remarks: endemic to the Danube drainage

PERCA Linnaeus, 1758

131. Perca fluviatilis Linnaeus, 1758

English name: Perch

Croatian name: grgeč

Range in Croatia: Danube drainage - middle and lower course of the rivers and lakes; Adriatic Basin - introduced to the Lika River system, Gacka River, Prološko Blato and waters of the Istrian Peninsula

Literature: Bonaparte (1832-1841); Šloser (1870); Brusina (1878); Jurinac (1880); Sebišanović (1880); Sebišanović (1881); Jurinac (1884); Mojsisovics (1884); Mojsisovics (1887); Hefele (1889); Sebišanović (1889b); Sebišanović (1889c); Sebišanović (1890); Brusina (1892); Franke (1892); Anonymous (1893d); Brusina (1893); Dudan 
(1893); Anonymous (1894c); Glowacki (1896); Hirc (1897); Hirc (1899e); Hirc (1902d); Ivakić (1905); RHZZM (1908); Ćurčić (1910); Hirc (1911); Kulčar (1911); Vutskits (1918); Plančić (1920); Anonymous (1925); Anonymous (1931c); Trojanović (1934); Jedlička (1935); Samobor (1935); Maldini (1936b); Maldini (1938c); Sabioncello (1953); Kosorić (1955); Jagodić (1957); Sabioncello (1967); Zobundžija (1968); Sabioncello et al. (1969); Vuković and Ivanović (1971); Herga (1968); Klašterka (1979); Mikuska (1979); Sofradžija and Hadžiselimović (1981); Bojčić et al. (1982); Veljović (1982); Mikuska (1983a); Mikuska (1983b); Delić (1984); Mikuska (1984); Mikuska and Mamić (1984); Vrenk (1984); Mikuska (1985); Tvrtković (1985); Veljović (1985); Habeković et al. (1986); Delić (1989); Habeković et al. (1990); Habeković and Popović (1991); Mrakovčić (1992); Delić (1993); Povž (1995); Zrnčić (1995); Mihaljević et al. (1996); Mrakovčić et al. (1996b); Habeković et al. (1997); Leiner (1998a); Majer (1998); Povž et al. (1998); Zanella et al. (2000); Jakovlić and Treer (2001); Mustafić (2001); Mrakovčić et al. (2002); Bakota et al. (2003); Delić et al. (2003a); Leiner (2003); Jugović (2004); Jakirčević (2005); Mustafić (2005); Pažur (2005); Suić (2005); Ćaleta (2007); Ivelić et al. (2007); Knežević (2007); Kottelat and Freyhof (2007); Prpa et al. (2007); Sallai and Mrakovčić (2007); Sallai and Kontos (2008); Sevcsik and Erös (2008); Treer et al. (2008a); Dumbović et al. (2009); Jelić et al. (2009); Kerestessy et al. (2009); Piria et al. (2009); Sofradžija (2009); Treer et al. (2009); Bučar et al. (2010); Buj (2010c); Jelić et al. (2010); Jelkić et al. (2010); Ozimec et al. (2010); Vardić Smrzlić (2010); Labak (2011); Matulić et al. (2011); Jelić et al. (2012); Marčić et al. (2012); Jelkić and Opačak (2013); Marr et al. (2013); Delić et al. (2014a); Nedić et al. (2014a); Nedić et al. (2014b); Riđanović et al. (2014); Piria et al. (2016a); Jelić et al. (2016); Pofuk et al. (2017); Simonović et al. (2017b); Nedić et al. (2018)

Remarks: non-native in the Adriatic Basin

\section{SANDER Oken, 1817}

\section{Sander lucioperca (Linnaeus, 1758)}

Names under which the taxon was mentioned: Lucioperca lucioperca (Linnaeus, 1758); Lucioperca sandra Cuvier, 1828; Stizostedion lucioperca (Linnaeus, 1758)

English name: Pike-perch

Croatian name: smuđ

Range in Croatia: Danube drainage - middle and lower course of the rivers and lakes; Adriatic Basin - introduced to the Butoniga and Tribalj reservoirs and Neretva River drainage

Literature: Heckel and Kner (1858); Brusina (1878); Jurinac (1880); Sebišanović (1880); Sebišanović (1881); Mojsisovics (1883); Jurinac (1884); Glowacki (1885); Anonymous (1886); Kesterčanek (1886); Seeley (1886); Jurinac (1887); Šoštarić (1888); Hefele (1889); Sebišanović
(1890); Brusina (1892); Franke (1892); Anonymous (1893d); Anonymous (1893d); Brusina (1893); Dudan (1893); Kišpatić (1893); Anonymous (1894c); Anonymous (1894d); Anonymous (1894g); Flögel (1894); Anonymous (1896a); Glowacki (1896); Anonymous (1897b); Mojsisovics (1897); Hirc (1899e); Steuer (1899); Aue (1900); Horvat (1900a); Horvat (1901); Medić (1901b); Langhoffer (1904); Anonymous (1905a); Anonymous (1905b); Ivakić (1905); Anonymous (1906b); Anonymous (1906d); Anonymous (1906e); Anonymous (1907a); Anonymous (1907b); Anonymous (1909b); Ćurčić (1910); Vutskits (1918); Plančić (1920); Plančić (1923a); Rössler (1928); Anonymous (1931c); Fink (1932a); Thaller (1932); Tadić (1933); Trojanović (1934); Bek (1935); Plančić (1935a); Samobor (1935); Anonymous (1936a); Maldini (1936a); Maldini (1937); Maldini (1938c); Anonymous (1946a); Anonymous (1947c); Hafner-Lahorski (1948b); Anonymous (1949); Fijan (1950); Hafner-Lahorski (1950a); Plančić (1952e); Sabioncello (1953); Marjanović (1954); Anonymous (1955b); Plančić (1955); Jagodić (1957); Salihćehajić (1965); Sabioncello (1967); Herga (1968); Zobundžija (1968); Anonymous (1969b); Tóth and Mikuska (1971); Vuković and Ivanović (1971); Munjko (1972); Pogrmilović (1972); Fijan (1976b); Klašterka (1979); Mikuska (1979); Sofradžija and Hadžiselimović (1981); Bojčić et al. (1982); Veljović (1982); Mikuska (1983a); Mikuska (1983b); Delić (1984); Mikuska (1984); Mikuska (1985); Tvrtković (1985); Veljović (1985); Habeković et al. (1986); Lelek (1987); Turk et al. (1987); Delić (1989); Mišetić et al. (1989); Treer (1989); Homen et al. (1991); Popović (1991); Martinović et al. (1992); Mrakovčić (1992); Delić (1993); Pažur (1993); Kolar-Dimitrijević (1994); Crivelli (1995); Zrnčić (1995); Krpo-Ćetković and Stamenković (1996); Mihaljević et al. (1996); Mrakovčić et al. (1996b); Leiner (1998a); Majer (1998); Holčík and Delić (2000); Zanella et al. (2000); Mustafić (2001); Mrakovčić et al. (2002); Delić et al. (2003a); Leiner (2003); Jugović (2004); Piria et al. (2004); Jakirčević (2005); Mustafić (2005); Pažur (2005); Mrakovčić et al. (2006); Ćaleta (2007); Ivelić et al. (2007); Kottelat and Freyhof (2007); Sallai and Mrakovčić (2007); Sallai and Kontos (2008); Sevcsik and Erös (2008); Suić et al. (2008); Jelić et al. (2009); Kerestessy et al. (2009); Sofradžija (2009); Jelić et al. (2010); Ozimec et al. (2010); Matulić et al. (2011); Jelić et al. (2012); Glamuzina et al. (2013); Jelkić and Opačak (2013); Marr et al. (2013); Zrnčić et al. (2013); Delić et al. (2014a); Nedić et al. (2014a); Nedić et al. (2014b); Riđanović et al. (2014); Pavličević et al. (2016); Piria et al. (2016b); Mustafić et al. (2017); Pofuk et al. (2017); Nedić et al. (2018); Piria et al. (2018)

Remarks: non-native in the Adriatic Basin

\section{Sander volgensis (Gmelin, 1789)}

Names under which the taxon was mentioned: Lucioperca volgensis (Gmelin, 1789); Stizostedion volgense (Gmelin, 1789) 


\section{English name: Volga pikeperch}

Croatian name: smuđ kamenjak

Range in Croatia: Danube drainage - Drava, Danube and Sava Rivers

Literature: Seeley (1886); Mojsisovics (1887); Hefele (1889); Brusina (1893); Kišpatić (1893); Glowacki (1896); Medić (1896); Hirc (1897); Medić (1901b); RHZZM (1908); Ćurčić (1910); Vutskits (1918); Maldini (1936a); Anonymous (1947c); Sabioncello (1953); Pešić (1955); Sabioncello (1967); Vuković and Ivanović (1971); Klašterka (1979); Mikuska (1979); Bojčić et al. (1982); Mikuska (1983a); Mikuska (1983b); Vrenk (1984); Lelek (1987); Homen et al. (1991); Harka (1992); Jakirčević (2005); Mustafić (2005); Pažur (2005); Mrakovčić et al. (2006); Kottelat and Freyhof (2007); Sallai and Mrakovčić (2007); Sallai and Kontos (2008); Sofradžija (2009); Ozimec et al. (2010); Jelić et al. (2012)

\section{ZINGEL Cloquet, 1817}

134. Zingel streber (Siebold, 1863)

Names under which the taxon was mentioned: Aspro streber Siebold, 1863; Aspro vulgaris Cuvier, 1828

English name: Streber

Croatian name: mali vretenac

Range in Croatia: Danube drainage - fast flowing parts of rivers

Literature: Heckel and Kner (1858); Jurinac (1880); Sebišanović (1880); Sebišanović (1881); Jurinac (1884); Glowacki (1885); Anonymous (1886); Kesterčanek (1886); Seeley (1886); Hefele (1889); Sebišanović (1890); Brusina (1893); Kišpatić (1893); Glowacki (1896); Hirc (1897); Mojsisovics (1897); Hirc (1899e); Medić (1901b); Langhoffer (1904); Ćurčić (1910); Vutskits (1918); Maldini (1935b); Sabioncello (1953); Sabioncello (1967); Vuković and Ivanović (1971); Klašterka (1979); Mikuska (1979); Bojčić et al. (1982); Veljović (1982); Mikuska (1983b); Popović and Fašaić (1986); Lelek (1987); Mrakovčić (1992); Mrakovčić et al. (1996b); Habeković et al. (1997); Majer (1998); Povž et al. (1998); Mustafić (2001); Jakirčević (2005); Mustafić (2005); Pažur (2005); Sallai and Kontos (2005); Mrakovčić et al. (2006); Kottelat and Freyhof (2007); Sallai and Mrakovčić (2007); Sallai and Kontos (2008); Dumbović et al. (2009); Sofradžija (2009); Ozimec et al. (2010); Popović (2010); Ištok (2011); Jelić et al. (2012); Delić et al. (2014a); Piria et al. (2016a)

135. Zingel zingel (Linnaeus, 1766)

Names under which the taxon was mentioned: Aspro zingel (Linnaeus, 1758)

English name: Zingel

Croatian name: veliki vretenac

Range in Croatia: Danube drainage - middle and lower course of the large rivers

Literature: Bonaparte (1846); Heckel and Kner (1858); Jurinac (1880); Sebišanović (1881); Jurinac (1884); Glowacki (1885); Seeley (1886); Sebišanović (1890); Brusina (1892); Brusina (1893); Dudan (1893); Kišpatić (1893); Glowacki (1896); Hirc (1897); Mojsisovics (1897); Hirc (1899e); Medić (1901b); RHZZM (1908); Ćurčić (1910); Vutskits (1918); Anonymous (1931c); Maldini (1935b); Sabioncello (1953); Sabioncello (1967); Vuković and Ivanović (1971); Klašterka (1979); Mikuska (1979); Bojčić et al. (1982); Veljović (1982); Mikuska (1983a); Mikuska (1983b); Lelek (1987); Harka (1992); Mrakovčić et al. (1996b); Majer (1998); Mustafić (2001); Mustafić (2005); Pažur (2005); Sallai and Kontos (2005); Mrakovčić et al. (2006); Ćaleta (2007); Kottelat and Freyhof (2007); Sallai and Mrakovčić (2007); Sallai and Kontos (2008); Sevcsik and Erös (2008); Kerestessy et al. (2009); Ozimec et al. (2010); Jelić et al. (2012); Piria et al. (2016a); Nedić et al. (2018)

\subsubsection{FAMILY: SPARIDAE Rafinesque, 1818}

SPARUS Linnaeus, 1758

136. Sparus aurata Linnaeus, 1758

English name: Silver seabream

Croatian name: komarča, orada

Range in Croatia: Adriatic Basin - river mouths, lagoons and lakes connected to the sea along the coast

Literature: Lorini (1921); Šoljan (1931); Morović (1947); Morović (1948); Basioli (1952); Kovačević (1952); Plančić (1952c); Taler (1953a); Basioli (1957a); Basioli (1958a); Basioli (1960); Morović (1961); Morović (1962); Marčić Brusina (1963); Morović (1963); Vuković (1963); Sabioncello et al. (1964); Habeković (1967); Vuković and Ivanović (1971); Morović (1976); Bojčić et al. (1982); Treer et al. (1989); Jardas (1996); Leiner (1998b); Schneider (1998); Sofradžija (2009); Dulčić and Glamuzina (2010); Lipej and Dulčić (2010); Tutman et al. (2010); Glamuzina et al. (2013)

Remarks: euryhaline species that occasionally enters freshwater

\subsubsection{FAMILY: GASTEROSTEIDAE Bonaparte, 1831}

\section{GASTEROSTEUS Linnaeus, 1758}

\section{Gasterosteus aculeatus Linnaeus, 1758}

Names under which the taxon was mentioned: Gasterosteus brachycentrus Cuvier, 1829; Gasterosteus gymnurus Cuvier, 1829

English name: Three-spined stickleback

Croatian name: koljuška

Range in Croatia: Danube drainage - introduced to Mura 
and Drava Rivers; Adriatic Basin - Mirna, Krka and Neretva River drainages, Baćinska Lakes, Vransko Lake near Biograd and Bokanjačko Blato Lake

Literature: Carrara (1846); Canestrini (1865); Canestrini (1866); Brusina (1878); Giglioli (1880); Seeley (1886); Katurić (1887); Mojsisovics (1887); Kolombatović (1888); Katurić (1896c); Katurić (1896d); Katurić 1907); Karaman (1928); Gridelli (1936); Taler (1953a); Pešić (1961); Vuković (1963); Vuković and Prolić (1966); Sabioncello (1967); Vuković and Kosorić (1967); Vuković and Ivanović (1971); Herga (1974); Morović (1976); Sofradžija and Hadžiselimović (1981); Bojčić et al. (1982); Leiner (1985); Lelek (1987); Fašaić et al. (1990); Mrakovčić and Mišetić (1990a); Leiner and Povž (1993); Mrakovčić et al. (1995); Jardas (1996); Maitland and Crivelli (1996); Holčík and Mrakovčić (1997); Leiner (1998b); Tvrtković (1998); Mrakovčić et al. (2000a); Mikavica et al. (2001); Paepke (2002); Mrakovčić et al. (2006); Kottelat and Freyhof (2007); Cano et al. (2008); Mäkinen and Merilä (2008); Muhamedagić et al. (2008); Sevcsik and Erös (2008); Zanella (2009); Zanella et al. (2009a); Lipej and Dulčić (2010); Csipkés et al. (2012); DeFaveri et al. (2012); Glamuzina et al. (2013); Landeka et al. (2015); Lisjak et al. (2015); Zanella et al. (2015); Piria et al. (2016b); Mustafić et al. (2017); Piria et al. (2018)

Remarks: non-native in Danube River drainage; though some report the existence of two species in Croatia, $G$. aculetus and $G$. gymnurus, recent genetic analyses have indicated that this is the species $G$. aculeatus

\section{ACKNOWLEDGEMENTS}

Authors are grateful to Linda Zanella for proofreading of the manuscript and two anonymous referees for their comments that improved the overall quality of the manuscript.

\section{PREGLED POSTOJEĆIH HRVATSKIH SLATKOVOD- NIH RIBA I PAKLARA}

\section{Popis i rasprostranjenost s komentarima}

\section{Sažetak}

Prvije put izrađen popis hrvatskih slatkovodnih riba. Temelji se na 1360 povijesnih i novijih literaturnih podataka. Na temelju pregleda literature, u Hrvatskoj je, do danas, zabilježeno 137 vrsta riba iz 30 porodica i 75 rodova. Popis je organiziran sistematski i u njemu se navode podaci o rasprostranjenosti slatkovodne ihtiofaune te jesu li vrste endemične, unesene ili prenesene (translocirane).

Ključne riječi: dunavski slijev, jadranski slijev, ednemičnost, unosi, prijenosi (translokacije).

\section{REFERENCES}

Aganović, M. (1957): Great Plivsko Lake. Ribarstvo Jugoslavije, 12, 1, 4-9. (in Croatian)

Aganović, M. (1979): Salmonid fish species and their breeding. OOUR Zavod za udžbenike, IGKRO Svjetlost, Sarajevo, 320pp. (in Croatian)

Aganović, M., Vuković, T. (1966): Relationship between length of the digestive tract and body length in three local populations of Dalmatian barbel gudgeon (Aulopyge hügelii Heck). Ribarstvo, 21, 1, 8-11. (in Croatian)

Almaca, C. (1984a): Notes on some species of Western Palearctic Barbus (Cyprinidae, Pisces). Arquivos do museu Bocage, 2, 1, 1-76.

Almaca, C. (1984b): Form relationship among Western Palearctic species of Barbus (Cyprinidae, Pisces). Arquivos do museu Bocage 2, 12, 207-248.

Al-Sabti, K. (1985): Chromosomal studies by blood leukocyte culture technique on three salmonids from Yugoslavian waters. Journal of Fish Biology, 26, 5-12.

Anonymous (1886): Ribarstvo, i rakolov. Izvješće Trgovačko-obrtničke komore u Zagrebu 1886. Trgovačka i obrtnička komora, Zagreb, 256-259.

Anonymous (1892): Fish farming of the Čabar estate. Viestnik prvog obćega Hrvatskoga društva za gojenje lova i ribarstva, 7, 79. (in Croatian)

Anonymous (1893a): Huchen in the Sava River near Zagreb. Viestnik prvog obćega Hrvatskoga društva za gojenje lova i ribarstva, 2, 15. (in Croatian)

Anonymous (1893b): Fishing in the Sava. Viestnik prvog obćega Hrvatskoga društva za gojenje lova i ribarstva, 2, 77. (in Croatian)

Anonymous (1893c): Communications. Viestnik prvog obćega Hrvatskoga društva za gojenje lova i ribarstva, 2, 106-107. (in Croatian)

Anonymous (1893d): Breeding of eels in Croatia. Viestnik prvog obćega Hrvatskoga društva za gojenje lova i ribarstva, 2, 126. (in Croatian)

Anonymous (1894a): Fishing on the Lonđa River. Lovačkoribarski viestnik, 3, 7-8. (in Croatian)

Anonymous (1894b): Communications. Lovačko-ribarski viestnik, 3, 9. (in Croatian)

Anonymous (1894c): Rare occurence in the fisheries world. Lovačko-ribarski viestnik, 3, 15. (in Croatian)

Anonymous (1894d): Pikeperch in the Sava. Lovačkoribarski viestnik, 3, 112. (in Croatian)

Anonymous (1894e): Communications. Lovačko-ribarski viestnik, 3, 122-124. (in Croatian)

Anonymous (1894f): Fisheries and crustacean harvesting in the area of the Senj trade and crafts chamber. Lovačko-ribarski viestnik, 3, 174-176. (in Croatian)

Anonymous (1894g): Rare fishes. Lovačko-ribarski viestnik, 3, 192. (in Croatian)

Anonymous (1895): Publication. Lovačko-ribarski viestnik, 4, 136. (in Croatian) 
Anonymous (1896a): Hunting and fishing revenues. Lovačko-ribarski viestnik, 5, 59. (in Croatian)

Anonymous (1896b): Trout in the streams around Zagreb. Lovačko-ribarski viestnik, 5, 59. (in Croatian)

Anonymous (1896c): Communications. Lovačko-ribarski viestnik, 5, 139-140. (in Croatian)

Anonymous (1897a): Fish farming on the Božjakovina estate. Lovačko-ribarski viestnik, 6, 23. (in Croatian)

Anonymous (1897b): Fishing details from Banovina. Lovačko-ribarski viestnik, 6, 72. (in Croatian)

Anonymous (1897c): Fish farming at the Plitvice Lakes. Lovačko-ribarski viestnik, 6, 96. (in Croatian)

Anonymous (1898a): Rare catch. Lovačko-ribarski viestnik, 7, 59. (in Croatian)

Anonymous (1898b): Breeding sturgeons from Croatia in the Rhone. Lovačko-ribarski viestnik, 7, 87. (in Croatian)

Anonymous (1899a): Zagreb Fisheries Society. Lovačkoribarski viestnik, 8, 23. (in Croatian)

Anonymous (1899b): Abundant fishing by hand. Lovačkoribarski viestnik, 8, 23. (in Croatian)

Anonymous (1899c): How do we breed fish?. Lovačkoribarski viestnik, 8, 47. (in Croatian)

Anonymous (1899d): For knowledge. Lovačko-ribarski viestnik, 8, 95. (in Croatian)

Anonymous (1900a): Rare catch in the Danube near Vukovar. Lovačko-ribarski viestnik, 9, 135. (in Croatian)

Anonymous (1900b): Rare catch. Lovačko-ribarski viestnik, 9, 136. (in Croatian)

Anonymous (1901): From the Zagreb fish market. Lovačkoribarski viestnik, 10, 106. (in Croatian)

Anonymous (1902): Breeding brook trout in the streams of the Zagreb hills. Lovačko-ribarski viestnik, 11, 82. (in Croatian)

Anonymous (1903a): Zagreb Fisheries Society. Lovačkoribarski viestnik, 12, 70. (in Croatian)

Anonymous (1903b): Huchen and „Lachsforelle“. Lovačkoribarski viestnik, 12, 71. (in Croatian)

Anonymous (1904): Grayling. Lovačko-ribarski viestnik, 13, 54-55. (in Croatian)

Anonymous (1905a): Sale of fish from Božjakovina. Lovačko-ribarski viestnik, 14, 45. (in Croatian)

Anonymous (1905b): Farming pikeperches and other fish species. Lovačko-ribarski viestnik, 14, 70. (in Croatian)

Anonymous (1905c): Carnivores and fish in the Croatian/ Slavonian Military Frontier one hundred years ago. Lovačko-ribarski viestnik, 14, 97-98. (in Croatian)

Anonymous (1906a): On the state of fisheries in the Dalmatian rivers. Lovačko-ribarski viestnik, 15, 23. (in Croatian)

Anonymous (1906b): Zagreb Fisheries Society. Lovačkoribarski viestnik, 15, 59. (in Croatian)

Anonymous (1906c): Fishing opportunities in the Ogulin and Slunj districts. Lovačko-ribarski viestnik, 15, 72. (in Croatian)

Anonymous (1906d): Fish farming in Croatia and Slavonia.
Lovačko-ribarski viestnik, 15, 119-120. (in Croatian)

Anonymous (1906e): A visit to a fish farm. Lovačko-ribarski viestnik, 15, 143. (in Croatian)

Anonymous (1907a): Fish farming on the Božjakovina estate. Lovačko-ribarski viestnik, 16, 47. (in Croatian)

Anonymous (1907b): For the purpose of breeding noble fishes in the Drava and Danube Rivers. Lovačko-ribarski viestnik, 16, 59. (in Croatian)

Anonymous (1907c): New trout stocking in the Ambrozovica stream. Lovačko-ribarski viestnik, 16, 120. (in Croatian)

Anonymous (1907d): For the purpose of breeding bullheads. Lovačko-ribarski viestnik, 16, 22-23. (in Croatian)

Anonymous (1908a): Rare catch. Lovačko-ribarski viestnik, 17, 47. (in Croatian)

Anonymous (1908b): Fish farming in Istria and Dalmatia. Lovačko-ribarski viestnik, 17, 47. (in Croatian)

Anonymous (1908c): Eels from Vrana Lake in Dalmatia. Lovačko-ribarski viestnik, 17, 70. (in Croatian)

Anonymous (1909a): The local history of hunting and fishing. Lovačko-ribarski viestnik, 18, 74-76. (in Croatian)

Anonymous (1909b): How to improve fisheries?. Lovačkoribarski viestnik, 18, 99-101. (in Croatian)

Anonymous (1910a): Bullheads in the Bednja River. Lovačko-ribarski viestnik, 19, 23. (in Croatian)

Anonymous (1910b): Eels in Lake Vrana on Cres Islands. Lovačko-ribarski viestnik, 19, 72. (in Croatian)

Anonymous (1911): Fishing success at Božjakovina. Lovačko-ribarski viestnik, 20, 11. (in Croatian)

Anonymous (1913): Rare catch. Lovačko-ribarski viestnik, 22, 72. (in Croatian)

Anonymous (1914): Salmon at the fish market in Zagreb. Lovačko-ribarski viestnik, 23, 34-36. (in Croatian)

Anonymous (1925): Sports exhibit. Angling. Ribarski vjesnik, 3, 8-9, 49-52. (in Croatian)

Anonymous (1926): Other. Športski ribar, 1, 4-5, 33-35. (in Croatian)

Anonymous (1927a): Grayling Športski ribar, 2, 1, 6-8. (in Croatian)

Anonymous (1927b): Other. Športski ribar, 2, 3-4, 43-44. (in Croatian)

Anonymous (1927c): Sporting news. Športski ribar, 2, 9-10, 114. (in Croatian)

Anonymous (1927d): From the angler's sac. Športski ribar, 2, 9-10, 115. (in Croatian)

Anonymous (1927e): Introduction of Gambusia patruelis in Italy. Rivista di Malariologia, 6, 4-5, 999-1000. (in Italian)

Anonymous (1927f): The Queen's Well. Ribarski vjesnik, 5, 9-10, 40. (in Croatian)

Anonymous (1927g): Rainbow trout. Ribarski vjesnik, 5, 9-10, 40. (in Croatian)

Anonymous (1927h): Maksimir Lake. Ribarski vjesnik, 5, 
9-10, 40. (in Croatian)

Anonymous (1927i): Huchen in the Sava River. Ribarski vjesnik, 5, 9-10, 40. (in Croatian)

Anonymous (1928a): From our waters. Ribarski list, 3, 3, 60-62. (in Croatian)

Anonymous (1928b): From the Adriatic. Ribarski list, 3, 3, 66-67. (in Croatian)

Anonymous (1928c): Fisheries on the Česma, Glogovnica and Lonja Rivers. Ribarski list, 3, 4, 88. (in Croatian)

Anonymous (1928d): Fishing in Ogulin waters. Ribarski list, 3, 4, 90. (in Croatian)

Anonymous (1928e): Are there eels in the Danube and its tributaries?. Ribarski list, 3, 4, 95-96. (in Croatian)

Anonymous (1928f): Opening trout season. Ribarski vjesnik, 6, 1-2, 8. (in Croatian)

Anonymous (1928g): Trout spawning. Ribarski vjesnik, 6, $1-2$, 8. (in Croatian)

Anonymous (1928h): Bitterling (Rhodeus amarus). Ribarski vjesnik, 6, 3-4, 14. (in Croatian)

Anonymous (1929a): Social news of the Osijek Angling Society. Ribarski list, 4, 1-2, 22-24. (in Croatian)

Anonymous (1929b): Large sterlet hooked from the Una. Ribarski list, 4, 3-4, 46. (in Croatian)

Anonymous (1929c): Trout in the tributaries of the Orljava. Ribarski list, 4, 3-4, 48-49. (in Croatian)

Anonymous (1929d): Swiss anglers in Ogulin. Ribarski list, 4, 5-6, 85. (in Croatian)

Anonymous (1929e): Migration and spawning of salmon, huchen and marble trout. Ribarski list, 4, 5-6, 86-88. (in Croatian)

Anonymous (1930a): Grayling - Thymalus vulgaris. Lovačko-ribarski vjesnik, 39, 83-84. (in Croatian)

Anonymous (1930b): Freshwater collossuses. Ribarski list, 5, 1-2, 18. (in Croatian)

Anonymous (1930c): From the Orljava. Ribarski list, 5, 3-4, 37. (in Croatian)

Anonymous (1930d): Marble trout Trutta genivittata C. Presp. in the Cetina. Ribarski list, 5, 3-4, 37-38. (in Croatian)

Anonymous (1930e): Breeding trouts in the Jadro River in Dalmatia. Ribarski list, 5, 7-8, 88-89. (in Croatian)

Anonymous (1930f): Dynamite fishers on the Una River. Ribarski list, 5, 9-12, 129. (in Croatian)

Anonymous (1930g): Neretva is a river of large trout. Ribarski list, 5, 9-12, 133-134. (in Croatian)

Anonymous (1930h): Great catch of $200 \mathrm{~kg}$ catfish. Ribarski vjesnik, 8, 11-12, 43. (in Croatian)

Anonymous (1931a): Giant catfish. Priroda, 21, 2-3, 91-92. (in Croatian)

Anonymous (1931b): Establishment of the fishing society in Sveti Ivan Zelina. Ribarski list, 6, 1-4, 29. (in Croatian)

Anonymous (1931c): Karlovac Fisheries Society. Ribarski list, 6, 1-4, 30-32. (in Croatian)

Anonymous (1931d): A $16 \mathrm{~kg}$ trout from the Plitvice Lakes. Ribarski list, 6, 1-4, 30-32. (in Croatian)
Anonymous (1931e): Lease of Vrana Lake. Ribarski list, 6, 7-10, 106. (in Croatian)

Anonymous (1931f): Trout for the royal court. Ribarski list, 6, 7-10, 108. (in Croatian)

Anonymous (1931g): Correspondence from Karlovac. Ribarski list, 6, 7-10, 108-109. (in Croatian)

Anonymous (1931h): A large breed of trout. Ribarski list, 6, 7-10, 113-114. (in Croatian)

Anonymous (1933): Fisheries concession on the Modruška Mrežnica. Ribarski list, 8, 5-6, 68. (in Croatian)

Anonymous (1934): Using fish to fight malaria. Ribarski list, 9, 8, 86. (in Croatian)

Anonymous (1935a): Lika Anglers' Society in Gospić. Ribarski vjesnik, 13, 3, 68-69. (in Croatian)

Anonymous (1935b): Unusually large trout from the Mrežnica. Ribarski vjesnik, 13, 3, 69. (in Croatian)

Anonymous (1935c): Ban on fishing in Lipovačka Gradna. Ribarski vjesnik, 13, 3, 71. (in Croatian)

Anonymous (1935d): First promotional fisheries fair. Ribarski vjesnik, 13, 3-4, 73-78. (in Croatian)

Anonymous (1935e): Good catch. Ribarski vjesnik, 13, 6, 141. (in Croatian)

Anonymous (1935f): Features of Lička Jasenica. Ribarski vjesnik, 13, 9-10, 244-245. (in Croatian)

Anonymous (1935g): Spawning of the Asp in the Drava River. Ribarski vjesnik, 13, 12, 300. (in Croatian)

Anonymous (1935h): Trout without red pigment. Ribarski vjesnik, 13, 12, 302. (in Croatian)

Anonymous (1935i): Improving freshwater fisheries in Savska Banovina. Ribarski vjesnik, 13, 1, 36-37. (in Croatian)

Anonymous (1935j): Zelina Fisheries Society. Ribarski vjesnik, 13, 1, 44-45. (in Croatian)

Anonymous (1936a): Successful catch. Ribarski vjesnik, 14, 2, 94. (in Croatian)

Anonymous (1936b): Pike - the devil. Ribarski vjesnik, 14, 4, 115-116. (in Croatian)

Anonymous (1936c): Huchen. Ribarski vjesnik, 14, 4, 120. (in Croatian)

Anonymous (1937a): Caught a $43 \mathrm{~kg}$ huchen. Ribarski vjesnik, 15, 1, 33. (in Croatian)

Anonymous (1937b): Local lexicon of the Drava Banovina. Uprava Krajevnega leksikona dravske banovine, Ljubljana. (in Croatian)

Anonymous (1939): Stocking trout in the Kamešnica in Kalnička Gora. Lovačko-Ribarski vjesnik, 48, 463. (in Croatian)

Anonymous (1945): Fish and crustaceans in the Plitvice Lakes. Priroda, 35, 9-10, 151-155. (in Croatian)

Anonymous (1946a): Fishing on the Una near Kostajnica. Gospodarski list, 16, 13. (in Croatian)

Anonymous (1946b): Trout farms in the People's Republic of Croatia. Ribarstvo Jugoslavije, 1, 2, 22. (in Croatian)

Anonymous (1947a): An $85 \mathrm{~kg}$ catfish. Ribarstvo Jugoslavije, 2, 4, 45. (in Croatian) 
Anonymous (1947b): An unusually large carp. Ribarstvo Jugoslavije, 2, 4, 46. (in Croatian)

Anonymous (1947c): Fishing opportunities in Brčko and the surrounding areas. Ribarstvo Jugoslavije, 2, 4, 46. (in Croatian)

Anonymous (1947d): Drying out part of Vrana Lake. Ribarstvo Jugoslavije, 2, 5, 103. (in Croatian)

Anonymous (1948a): Stray trout. Ribarstvo Jugoslavije, 3, 7-8, 76. (in Croatian)

Anonymous (1948b): An 18 kg carp. Ribarstvo Jugoslavije, 3, 7-8. 78. (in Croatian)

Anonymous (1948c): Large catfish on the Sava River. Ribarski list, 6-7, 11. (in Croatian)

Anonymous (1948d): The hunt for giant fish. Ribarski list, 11-12, 12. (in Croatian)

Anonymous (1949): Development and work of the Zagreb Fisheries Society. Ribarstvo Jugoslavije, 4, 1-2, 19. (in Croatian)

Anonymous (1952a): Stocking the Dretulja River in Plaško. Ribarstvo Jugoslavije, 7, 4, 60. (in Croatian)

Anonymous (1952b): Stocking the Odra with trout. Ribarstvo Jugoslavije, 7, 7, 125. (in Croatian)

Anonymous (1952c): Unexpected and rare success on the Kupa. Ribarstvo Jugoslavije, 7, 11-12, 204. (in Croatian)

Anonymous (1953a): Perhaps you didn't know. Ribarstvo Jugoslavije, 8, 3, 67-68. (in Croatian)

Anonymous (1953b): A rare catch of a sturgeon. Ribarstvo Jugoslavije, 8, 4, 89-90. (in Croatian) Anonymous (1955a): Hybrids of the rainbow trout. Ribarski list, 3, 69. (in Croatian)

Anonymous (1955b): Eel captured in the waters of the Danube. Ribarski list, 6, 136.

Anonymous (1955c): From our association. Ribarski list, 6, 138-139. (in Croatian)

Anonymous (1958a): In the Gacka River, the trouts grow very fast. Ribič, 2, 63. (in Croatian)

Anonymous (1958b): From the angler's sac. Ribarski list, 2-3, 63-64. (in Croatian)

Anonymous (1959): Small news. Ribarski list, 1, 23-24. (in Croatian)

Anonymous (1964): Investigations of Vrana Lake. Ribarstvo Jugoslavije, 19, 4, 81. (in Croatian)

Anonymous (1965a): Our waters are rich in fish species. Ribarski list, 4, 76. (in Croatian)

Anonymous (1965b): Trout caught in the Adriatic Sea. Ribarski list, 5, 105. (in Croatian)

Anonymous (1965c): Eels in the Sava, Danube and Tisa Rivers. Ribarski list, 5, 107. (in Croatian)

Anonymous (1967): Cadastre of the fishing waters of the Socialist Republic of Croatia - Adriatic Basin. Institut za slatkovodno Ribarstvo, Zagreb, 74pp. (in Croatian)

Anonymous (1968): From other republics. Ribarski list, 3, 84. (in Croatian)

Anonymous (1969a): Catch of the huchen near Donji Miholjac. Ribarstvo Jugoslavije, 24, 1, 18. (in Croatian)
Anonymous (1969b): Short news. Ribarski list, 4, 95. (in Croatian)

Asaj, A., Šalić, R. (1957): Causes of fish gasping in Bosut River. Veterinarski arhiv, 3-4, 95-105. (in Croatian)

Aspacher, B., Haslinger, R., Meyer, U., Oertel, A. (2000): Beyond the blue. NSS News, 58, 5, 141-149.

Aue, J. (1900): +Is the fishing ban during fish spawning still in effect!. Lovačko-ribarski viestnik, 9, 61. (in Croatian)

Babajko, M. (2011): Una is the only one. Hrvatska vodoprivreda 194, 88-92. (in Croatian)

Babić, M. (2016): The underwater world of Lake Vrana on Cres Island. Hrvatska vodoprivreda 217, 93-100. (in Croatian)

Bajić, S. (1927): Pike. Športski ribar, 2, 3-4, 26-27. (in Croatian)

Bajić, S. (1928): Catfish. Ribarski list, 3, 6, 122-125. (in Croatian)

Bakić, J. (2006): Credible facts regarding the first medical entomological laboratory and the introduction of Gambusia holbrooki in Croatia. Hrvatski časopis za javno zdravstvo, 2, 6, 1-5. (in Croatian)

Bakić, J. (2011): Seven centuries in combatting the introduction of infections into Croatia - A reveiw of the $60^{\text {th }}$ anniversary of the organisation of the contemporary DDD activities in Croatia. Hrvatski časopis za javno zdravstvo, 7, 25, 1-17. (in Croatian)

Bakota, R., Treer, T., Odak, T., Mrakovčić, M., Ćaleta, M. (2003): Structure and condition of the icthyofauna of Lonjsko Polje. Ribarstvo, 61, 1, 17-26. (in Croatian)

Balbo, A. L. (2005): The last days of a Mediterranean wet landscape: Human impact on and draining of shallow lake Arsa, Polje Čepić, Istria, Croatia. $10^{\text {th }}$ UNESCO Universities Heritage Forum, Newcastle, 1-9.

Bănărescu, P. M. (1973): Origin and affinities of the freshwater fish fauna of Europe. Ichthyologia, 5, 1, 1-8.

Bănărescu, P. M. (1999a): Gobio kessleri Dybowski, 1862. In: Bănărescu, P. M. (ed), The Freshwater Fishes of Europe. Cyprinidae 2/I. Vol. 5/I. AULA-Verlag, Wiebelsheim, 135-162.

Bănărescu, P. M. (1999b): Pseudorasbora parva (Temminck et Schlegel, 1846). In: Bănărescu, P. M. (ed), The Freshwater Fishes of Europe. Cyprinidae 2/I. Vol. 5/I. AULA-Verlag, Wiebelsheim, 207-224.

Bănărescu, P. M., Bless, R., Economidis, P. S. (1999b): Gobio uranoscopus (Agassiz, 1828). In: Bănărescu, P. M. (ed), The Freshwater Fishes of Europe. Cyprinidae 2/I. Vol. 5/I. AULA-Verlag, Wiebelsheim, 183-202.

Bănărescu, P. M., Bogutskaya, N. G., Movchan, Y., Smirnov, A. (2003): Barbus barbus. In: Bănărescu, P. M., Bogutskaya, N. G. (eds), The Freshwater Fishes of Europe, Cyprinidae 2/II. Vol 5/II. AULA-Verlag, Wiebelsheim, 43-98.

Bănărescu, P. M., Herzig-Straschil, B. (1998): Beitrag zur Kenntnis der Leuciscus-Untergattung Telestes Bonaparte (Pisces: Cyprinidae). Annalen des Naturhistorischen Museums in Wien, 100B, 405-424. 
(in German)

Bănărescu, P. M., Šorić, V. M., Economidis, P. S. (1999a): Gobio gobio (Linnaeus, 1758). In: Bănărescu, P. M. (ed), The Freshwater Fishes of Europe. Cyprinidae 2/l. Vol. 5/I. AULA-Verlag, Wiebelsheim, 81-134.

Bănărescu, P. M., Nalbant, T. T. (1973): Pisces, Teleostei. Cyprinidae (Gobioninae). Das Tierreich. Eine Zusmmenstellung und Kenneichnung der rezenten Tierformen 93, 1-304. (in German)

Barišić, J., Filipović Marijić, V., Mijošek, T., Čož-Rakovac, R., Dragun, Z., Krasnići, N., Ivanković, D., Kružlicová, D., Erk, M. (2018): Evaluation of architectural and histopathological biomarkers in the intestine of brown trout (Salmo trutta Linnaeus, 1758) challenged with environmental pollution. Science of the Total Environment, 642, 656-664.

Bartulović, V., Dulčić, J., Bogut, I., Pavličević, J., Hasković, E., Glamuzina, B. (2011): First record of the freshwater bream, Abramis brama in the river Mala Neretva, Adriatic drainage system of Croatia. Cybium, 35, 2, 165166.

Bartulović, V., Glamuzina, B., Lučić, D., Conides, A., Jasprica, N., Dulčić, J. (2007): Recruitment and food composition of juvenile thin-lipped grey mullet, Liza ramada (Risso, 1826) in the Neretva River estuary (Eastern Adriatic, Croatia). Acta Adriatica, 1, 25-37.

Bartulović, V., Lučić, D., Zlatović, A., Dobroslavić, T., Tomić, S., Glamuzina, B. (2010): Recruitment of juvenile Golden grey mullet, Liza aurata (Risso, 1810) and Flathead grey mullet, Mugil cephalus Linnaeus, 1758 in the Neretva River estuary. Ribarstvo, 68, 2, 61-78. (in Croatian with English abstract)

Baruš, V., Bănărescu, P. M., Kohlmann, K. (2002): Cyprinus carpio. In: Bănărescu, P. M., Paepke HJ (eds), The Freshwater Fishes of Europe. Cyprinidae 2/III. Vol 5/III. AULA-Verlag, Wiebelsheim, 85-179.

Basioli, J. (1952): The decline of fish catches in the lower Neretva area. Morsko Ribarstvo, 4, 1-2, 12-15. (in Croatian)

Basioli, J. (1957a): Fisheries of the Neretva River. Ribarstvo Jugoslavije, 12, 3, 43-46. (in Croatian)

Basioli, J. (1957b): Fisheries of the island of Cres. Morsko Ribarstvo, 9, 3, 81-84. (in Croatian)

Basioli, J. (1958a): Fisheries in the lower Neretva area. Morsko Ribarstvo, 10, 12, 263-265. (in Croatian)

Basioli, J. (1958b): Fisheries of the Krka River. Ribarstvo Jugoslavije, 13, 5, 105-107. (in Croatian)

Basioli, J. (1959): Fisheries of the Dubrovnik region. Morsko Ribarstvo, 11, 10, 222-225. (in Croatian)

Basioli, J. (1960): Fishing on Vrana Lake. Ribarstvo Jugoslavije, 15, 5, 131-132. (in Croatian)

Basioli, J. (1971): Eels (Anguillidae). Morsko Ribarstvo, 23, 4, 157-168. (in Croatian)

Bašić, Đ. (2005): Fishing in Dalmatia in the 19th and 20th centuries. Pomorski zbornik, 43, 1, 261-283. (in Croatian with English abstract)
Bath, H. (2003): Salaria fluviatilis (Asso, 1784). In: Miller JP (ed), The Freshwater Fishes of Europe. Mugilidae, Atherinidae, Atherinopsidae, Blenniidae, Odontobutidae, Gobiidae 1. Vol 8/I. AULA-Verlag, Wiebelsheim, 97-121.

Bauer, J. (1983): Current problems in Lonjsko Polje. Ribarstvo Jugoslavije, 38, 2, 32-37. (in Croatian)

Behnke, R. J. (1968): A new subgenus and species of trout, Salmo (Platysalmo) platycephalus, from southcentral Turkey, with comments on the classification of the subfamily Salmoninae. Mitteilungen aus dem Hamburgischen Zoologischen Museum und Institut, 66, 1-15.

Bek, B. (1935): With harpoons on the Una River. Lovačkoribarski vjesnik, 44, 415-417. (in Croatian)

Berberović, Lj., Hadžiselimović, R., Pavlović, B., Sofradžija, A. (1971): General comparative data on the chromosome setup of the species Paraphoxinus adspersus Heckel 1843 and Paraphoxinus croaticus Steindachner 1865. Ichthyologia, 3, 1, 3-11. (in Croatian)

Berg, L. S. (1932a): Les poissons des eaux douces de I'U.R.S.S. et des pays limitrophes. 3-e édition, revue et augmentée. Les poissons des eaux douces de I'U.R.S.S. Part 1. Leningrad, 543pp. (in French)

Berg, L. S. (1948-1949): Ryby Presnykh vod SSSR i sopredel'nykh stran.4 ed 1 izd. Akademii Nauk SSSR, Moskva-Leningrad, 505pp. (in Russian)

Bergleitner, R. (1925): Osijek angling society. Ribarski vjesnik, 3, 8-9, 54. (in Croatian)

Bergleitner, R. (1928): From the Ogulin waters. Ribarski list, 3, 1-2, 14-16. (in Croatian)

Bergleitner, R. (1929): Large trout from the Mrežnica near Ogulin. Ribarski list, 4, 7-10, 113-114. (in Croatian)

Bergleitner, R. (1930): From the angler's sac. Ribarski list, 4, 9-12, 132-134. (in Croatian)

Bergleitner, R. (1940): Grayling (Thymallus thymallus Linné). Priroda, 30, 8, 236-241. (in Croatian)

Bergleitner, R. (1946): Crustaceans as food for trout in the Ogulin waters. Ribarstvo Jugoslavije, 1, 1, 10. (in Croatian)

Berinkey, L. (1966): Halak-Pisces. Fauna Hungariae, 79, 1-139. (in Hungarian)

Bernatchez, L., Guyomard, R., Bonhomme, F. (1992): DNA sequence variation of the mitochondrial control region among geographically and morphologically remote European brown trout Salmo trutta populations. Molecular Ecology, 1, 161-173.

Bernatchez, L. (2001): The evolutionary history of brown trout (Salmo trutta L.): inferred from phylogeographic, nested clade, and mismatch analyses of mitochondrial DNA variation. Evolution, 55, 351-379.

Bianco, P. G. (1986): The zoogeographic units of Italy and western Balkans based on cyprinid species ranges (Pisces). Biologia Gallo-Hellenica, 12, 291-299.

Bianco, P. G. (1995a): Mediterranean endemic freshwater fishes of Italy. Biological conservation, 72, 159-170. 
Bianco, P. G. (1995b): A revision of the Italian Barbus species (Cypriniformes; Cyprinidae). Ichthyological exploration of freshwaters, 6, 4, 305-324.

Bianco, P. G. (1998): Diversity of Barbine fishes in southern Europe with description of a new genus and a new species (Cyprinadae). Italian Journal of Zoology 65, 125-136.

Bianco, P. G. (2002): The Status of the Twaite Shad, Alosa agone, in Italy and the Western Balkans. Marine Ecology, 23 (Supplement 1), 51-64.

Bianco, P. G. (2003): Barbus plebejus, In: Bănărescu, P. M., Bogutskaya, N. G. (eds), The freshwater fishes of Europe, Cyprinidae 2/II. Vol 5/II. AULA-Verlag, Wiebelsheim, 339-364.

Bianco, P. G. (2014a): An update on the status of native and exotic freshwater fishes of Italy. Journal of Applied Ichthyology, 30, 62-77.

Bianco, P. G. (2014b): Aggiornamento sistematico dei pesci d'acqua dolce autoctoni Italiani: proposta di un workshop. Italian Journal of Freshwater Ichthyology, 1, 133-162. (in English)

Bianco, P. G., De Bonis, S. (2015): A taxonomic study on the genus Phoxinus (Actinopterigy, Cyprinidae) from Italy and western Balkans with description of four new species: $P$. ketmaieri, $P$. karsticus, $P$. apollonicus and $P$. likai. Researches on Wildlife Conservation, 4, 1-17.

Bianco, P. G., Ketmaier, V. (2014): A revision of the Rutilus complex from Mediterranean Europe with description of a new genus, Sarmarutilus, and a new species, Rutilus stoumboudae (Teleostei: Cyprinidae). Zootaxa, 3841, 3, 379-402.

Bianco, P. G., Ketmaier, V. (2015): Nature and status of freshwater and estuarine fisheries in Italy and Western Balkans. In: Craig JF (ed), Freshwater Fisheries Ecology. John Wiley \& Sons, Ltd, Chichester, UK, 283-291.

Bianco, P. G., Knežević, B. (1987): The Leuciscus cephalus complex (Pisces, Cyprinidae): in the western Balcanic area. In: Kullander, S. O., Fernholm, B. (eds), Proceedings of V. Congress of European Ichthyologists, Stockholm, 49-55.

Bianco, P. G., Miller, P. J. (1990): Yugoslavian and other records of the Italian freshwater goby, Padogobius martensii, and a character polarization in gobioid fishes. Journal of Natural History, 24, 1289-1302.

Bianco, P. G., Taraborelli, T. (1985): Contributo alla conoscenza del genere Rutilus Rafinesque in Italia e nei Balcani occidentali (Pisces, Cyprinidae). Bollettino del Museo Regionale di Scienze Naturali di Torino, 3, 131172. (in Italian)

Biber, E. S. (Ur.): (1997): Chronicle of the Franciscan monastery in Brod on Sava II (1806.-1833.). Matica hrvatska, Slavonski Brod. 453pp. (in Croatian)

Bikić, M. (1966): Fish smell of petrol. Ribarski list, 17, 1. (in Croatian)

Bilopavlović, V. (1970): Gaovica in the waters of Imotski. Priroda, 57, 7, 221-222. (in Croatian)
Bleeker, P. (1863): Atlas ichthyologique des Indes Orientales Néêrlandaises. Tome III. Cyprins. Frédéric Muller, Amsterdam, 150pp. (in French)

Bless, R., Riehl, R. (2002): Biology and egg morphology of the Dalmatian Barbelgudgeon Aulopyge huegeli, an endangered endemic species in Croatia. Environmental Biology of Fishes, 63, 4, 451-456.

Bloesch, J., Jones, T., Reinartz, R., Striebel, B. (2006a): An action plan for the conservation of sturgeons (Acipenseridae): in the Danube River Basin. Österreichische Wasser und Abfallwirtschaft, 58,5, 8188.

Bloesch, J., Jones, T., Reinartz, R., Striebel, B. (2006b): Action Plan for the conservation of Sturgeons (Acipenseridae): in the Danube River Basin. Convention on the Conservation of European Wildlife and Natural Habitats (Bern Convention). Nature and Environment, 144, 1-122.

Bogdanović, I. (1957): Small stream - rich fishing area. Ribarstvo Jugoslavije, 12, 3, 62. (in Croatian)

Bogdanović, I. (1959): Problems concerning the stocking of the Plitvice Lakes. Ribarstvo Jugoslavije, 14, 4, 94-95. (in Croatian)

Bogdanović, I. (1961): First serious steps in the stocking of the Plitvice Lakes. Ribarstvo Jugoslavije, 16, 2, 44-46. (in Croatian)

Bogdanović, J. (1954): In the waters of the Plitvice catchment. Ribarstvo Jugoslavije, 2, 9, 35-37. (in Croatian)

Bogdanović, J. (1958): Rainbow trout in the Plitvice Lakes. Ribarstvo Jugoslavije, 13, 6, 133-134. (in Croatian)

Bogut, I., Magovac, R., Sabo, D., Bodakoš, D., Galović, D., Arežina, M., Rajković, V. (2007): Cage fattening results of Common carp (Cyprinus carpio): in hydroaccumulation grabovo near Vukovar. Krmiva, 4, 207-214. (in Croatian with English abstract)

Bogutskaya, N. G., Zupančič, P. (1999): A re-description of Leuciscus zrmanjae (Karaman, 1928): and new data on the taxonomy of Leuciscus illyricus, L. svallize and L. cephalus (Pisces: Cyprinidae): in the West Balkans. Annalen des Naturhistorischen Museums in Wien, 101(B), 509-529.

Bogutskaya, N. G., Zupančič, P. (2003): Phoxinellus pseudalepidotus (Teleostei: Cyprinidae): a new species from the Neretva basin with an overview of the morphology of Phoxinellus species of Croatia and Bosnia-Herzegovina. Ichthyological Exploration of Freshwaters, 14, 4, 369-383.

Bogutskaya, N. G., Zupančič, P. (2010): Squalius janae, a new species of fish from the Adriatic Sea basin in Slovenia (Teleostei: Cyprinidae). Zootaxa, 2536, 53-68.

Bogutskaya, N. G., Zupančič, P., Bogut, I., Naseka, A. M. (2012): Two new freshwater fish species of the genus Telestes (Actinopterygii, Cyprinidae): from karst poljes in eastern Herzegovina and Dubrovnik littoral (Bosnia and Herzegovina and Croatia). ZooKeys, 180, 53-80. 
Bogutskaya, N. G., Zupančič, P., Jelić, D., Diripasko, O. A., Naseka, A. M. (2017): Description of a new species of Alburnus Rafinesque, 1820 (Actinopterygii, Cyprinidae, Leuciscinae) from the Kolpa River in the Sava River system (upper Danube drainage): with remarks on the geographical distribution of shemayas in the Danube. ZooKeys, 688, 81-110.

Bohlen, J., Ráb, P. (2001): Species and hybrid richness in spined loaches (genus Cobitis L.) with a checklist of the species and hybrids of Europe. Journal of Fish Biology, 59A, 75-89.

Böhm, V. (1929): From our waters. Ribarski list, 3-4, 46-49. (in Croatian)

Bojčić, C. (1987): Development strategy for carp fisheries based on a greater share of secondary fish in total production. Ribarstvo Jugoslavije, 42, 6, 130-140. (in Croatian)

Bojčić, C., Debeljak, Lj., Vuković, T., Jovanović-Kršljanin, B., Apostolski, K., Ržaničanin, B., Turk, M., Volk, S., Drecun, Đ., Habeković, D., Hristić, Đ., Fijan, N., Pažur, K., Bunjevac, I., Marošević, Đ. (1982): Freshwater fisheries. Poslovna zajednica slatkovodnog ribarstva Jugoslavije, Zagreb, 605pp. (in Croatian)

Bonacci, O., Kerovec, M., Roje-Bonacci, T., Mrakovčić, M., Plenković-Moraj, A. (1998): Ecologically acceptable flows definition for the Žrnovnica River (Croatia). Regulated rivers, Research \& Management, 14, 245256.

Bonaparte, C. L. P. (1832-1841): Tomo III. Pesci. Iconografia della fauna Italica per le quattro classi degli animali vertebrati. Salviucci, Roma, 556pp. (in Italian)

Bonaparte, C. L. P. (1845): Catalogo metodico dei ciprinidi d'Europa. Pirola, Milano, 20pp. (in Italian)

Bonaparte, C. L. P. (1845): Catalogo metodico dei pesci Europei. Fibreno, Napoli, 97pp. (in Italian)

Bönel, V. (1901): Hunting and fishing on the Čabar estate. Lovačko-ribarski viestnik, 10, 92-93. (in Croatian)

Borne, M. (1881): Die Fischerei-Verhältnisse des Deutschen Reiches, Oesterreich-Ungarns, der Schweiz und Luxemburgs bearbeitet Auftrage des Deutschen Fischerei-Vereins. W. Moeser Hofbuchdruckerei, Berlin, 303pp. (in German)

Bošnir, J., Puntarić, D., Klarić, M., Šmit, Z. (2005): Polychlorinated biphenyls in freshwater fish from the Zagreb area. Arhiv za higijenu rada i toksikologiju, 4, 303-309.

Bošnir, J., Puntarić, D., Škes, I., Klarić, M., Šimić, S., Zorić, I., Galić, R. (2003): Toxic metals in freshwater fish from the Zagreb area as indicators of environmental pollution. Collegium Antropologicum, 27, (Supplement 1), 31-39.

Bošnir, J., Puntarić, D., Šmit, D., Klarić, M., Grgić, M., Kosanović, L. M. (2007): Organochlorine pesticides in freshwater fish from the Zagreb Area. Arhiv za higijenu rada i toksikologiju, 2, 187-193.

Botteri, M. (1891): Two lists of Dalmatian fish by M. Botteri, with additions by Heckel, Bellotti, Stalio and others, with introduction by S. Brusina. Glasnik Hrvatskoga naravoslovnoga društva, 1-5, 109-151. (in Croatian)

Bristol, J. R., Mayberry, L. F , Huber, Đ., Ehrlich, I. (1984): Endoparasite fauna of trout in the Plitvice Lakes National Park. Veterinarski arhiv, 54, 5-11.

Brusina, S. (1872): Natural history of the northeastern coast of the Adriatic Sea: collected in 1868 and 1871. Part one. Rad Jugoslavenske akademije znanosti i umjetnosti 19, 105-178. (in Croatian)

Brusina, S. (1874): Natural history of the northeastern coast of the Adriatic Sea. Part two. Rad Jugoslavenske akademije znanosti i umjetnosti, 27, 131-193. (in Croatian)

Brusina, S. (1878): Animals of Croatia. In: Klaić V (ed), Prirodni zemljopis Hrvatske. Albrecht, Zagreb, 378-397. (in Croatian)

Brusina, S. (1880): One decade of our zoological literature: (1867-1877). Rad Jugoslavenske akademije znanosti i umjetnosti, 52, 190-272. (in Croatian)

Brusina, S. (1889): "Milk“ fish amongst Dalmatians. Glasnik Hrvatskoga naravoslovnoga družtva, 1-5, 5667. (in Croatian)

Brusina, S. (1890): Invitation of the department for a fisheries jubille commerical-forestry exhibit. Glasnik Hrvatskoga naravoslovnoga družtva, 1-4, 251-257. (in Croatian)

Brusina, S. (1892): Scraps for Croatian ichthyology and for fisheries. Glasnik Hrvatskoga naravoslovnoga društva, 1-6, 221-288. (in Croatian)

Brusina, S. (1893): In protection of fisheries. Viestnik prvog obćega Hrvatskoga društva za gojenje lova i ribarstva, 1, 36-42. (in Croatian)

Brusina, S. (1897): Green pike. Lovačko-ribarski viestnik, 6, 117. (in Croatian)

Brusina, S. (1898): Stickebacks (Gasterosteus) from Serbia. Glasnik Hrvatskoga naravoslovnoga društva, 1-5, 209212. (in Croatian)

Brusina, S. (1899): European mudminnow on the Zagreb fish market. Lovačko-ribarski viestnik, 8, 105. (in Croatian)

Brusina, S. (1902): Our sturgeons. Rad Jugoslavenske akademije znanosti i umjetnosti, 149, 31, 1-69. (in Croatian)

Brusina, S. (1907): Natural history of the northeastern coast of the Adriatic Sea. Part four and final. Special. Rad Jugoslavenske akademije znanosti i umjetnosti, 171, 43-228. (in Croatian)

Brusina, S. (1908): Natural history of the northeastern coast of the Adriatic Sea. Rad Jugoslavenske akademije znanosti i umjetnosti, 43, 1-31. (in Croatian)

Bryja, J., Smith, C., Konečný, A., Reichard, M. (2010): Rangewide population genetic structure of the European bitterling (Rhodeus amarus): based on microsatellite and mitochondrial DNA analysis. Molecular Ecology, 19, 4708-4722.

Brylińska, M., Bryliński, E., Bnińska, M. (1999): Tinca 
tinca (Linnaeus, 1758). In: Bănărescu, P. M. (ed), The Freshwater Fishes of Europe. Cyprinidae 2/I. Vol. 5/l. AULA-Verlag, Wiebelsheim, 229-302.

Buchwald, S. (1896): Die Plitvicer Seen und ihr Vorland. A. Reinhard's Verlag, Rijeka, 65pp. (in German)

Bučar, M., Delić, A., Kučinić, M., Vilenica, M. (2010): Lampreys and fishfauna of the flowing waters of Mount Zrin. In: Bučar, M. (ed), Zrinska Gora Regional Nature Park. Grad Petrinja, Sisačko-Moslavačka županija, Sveučilište u Zagrebu Učiteljski fakultet Odsjek Petrinja, Matica hrvatska ogranak Petrinja, Petrinja, 198-207. (in Croatian)

Buj, I. (2010a): Taxonomic position, phylogenetic relations and morphological characters of spined loaches of the genus Cobitis (Cypriniformes; Actinopterygii) distributed in Dalmatia and Herzegovina. PhD thesis, Faculty of Science, University of Zagreb. (in Croatian with English abstract)

Buj, I. (2010b): A mysterious and undiscovered world, Gorski Kotar, the last oasis of endemic fishes in the Danube Basin. Euro city, putna revija HŽ-putničkog prijevoza, 18, 67, 100-101. (in Croatian)

Buj, I. (2010c): World record trout, a haven for anglers. Euro city, putna revija HŽ-putničkog prijevoza, 18, 67, 103. (in Croatian)

Buj, I., Ćaleta, M., Marčić, Z., Šanda, R., Vukić, J., Mrakovčić, M. (2015c): Different histories, different destiniesimpact of evolutionary history and population genetic structure on extinction risk of the Adriatic spined loaches (genus Cobitis, Cypriniformes, Actinopterygii). PLOS ONE, 10, 7, 1-17.

Buj, I., Marčić, Z., Čavlović, K., Ćaleta, M., Tutman, P., Zanella, D., Duplić, A., Raguž, L., Ivić, L., Horvatić, S., Mustafić, P. (2019): Multilocus phylogenetic analysis helps untangling the taxonomic puzzle of chubs (genus Squalius; Cypriniformes, Actinopteri) in the Adriatic basin in Croatia and Bosnia and Herzegovina. Zoological Journal of the Linnean Society, in press

Buj, I., Miočić-Stošić, J., Marčić, Z., Mustafić, P., Zanella, D., Mrakovčić, M., Mihinjač, T., Ćaleta, M. (2015b): Population genetic structure and demographic history of Aphanius fasciatus (Cyprinodontidae, Cyprinodontiformes) from hypersaline habitats in the eastern Adriatic. Scientia marina, 79, 4, 399-408.

Buj, I., Podnar, M., Mrakovčić, M., Choleva, L., Šlechtova, V., Tvrtković, N., Ćaleta, M., Mustafić, P., Marčić, Z., Zanella, D., Brigić, A. (2008b): Genetic diversity and phylogenetic relationships of spined loaches (genus Cobitis) in Croatia based on mtDNA and allozyme analyses. Folia Zoologica, 57, 1-2, 71-82.

Buj, I., Podnar, M., Mrakovčić, M., Ćaleta, M., Mustafić, P., Zanella, D., Marčić, Z. (2008a): Morphological and genetic diversity of Sabanejewia balcanica in Croatia. Folia Zoologica, 5, 1-2, 100-110.

Buj, I., Šanda, R., Marčić, Z., Ćaleta, M., Mrakovčić, M. (2014): Combining morphology and genetics in resolving taxonomy - a systematic revision of spined loaches (genus Cobitis, Cypriniformes, Actinopterygii) in the Adriatic watershed. PLOS ONE, 9, 6, 1-25.

Buj, I., Šanda, R., Marčić, Z., Ćaleta, M., Mrakovčić, M. (2015a): Sexual dimorphism of five Cobitis species (Cypriniformes, Actinopterygii): in the Adriatic watershed. Folia Zoologica, 64, 2, 97-103.

Buj, I., Vukić, J., Šanda, R., Perea, S., Ćaleta, M., Marčić, Z., Bogut, I., Povž, M., Mrakovčić, M. (2010): Morphological comparison of bleaks (Alburnus, Cyprinidae): from the Adriatic Basin with the description of a new species. Folia Zoologica, 59, 2, 129-141.

Buj, I., Marčić, Z., Ćaleta, M., Šanda, R., Geiger, M. F., Freyhof, J., Machordom, A., Vukić, J. (2017): Ancient connections among the European rivers and watersheds revealed from the evolutionary history of the genus Telestes (Actinopterygii; Cypriniformes). PLOS ONE, 12, 12, e0187366.

Bukvić, V., Bogut, I., Galović, D. (2010): Heavy metals in fish tissues in Neretva River Delta. In: Glamuzina, B., Dulčić, J. (eds), Fish and Fisheries of the Neretva River. Sveučilište u Dubrovniku i Dubrovačko neretvanska županija, Dubrovnik, 154-159. (in Croatian with English abstract)

Bullo, F. (1891): Piscicultura marina, stima delle coltivacioni in aqua salsa. Parte I: Notizie preliminari. Stabilimento Prosperini, Padova, 61pp. (in Italian)

Canestrini, G. (1865): Note Ittiologiche. Archivio per la Zoologia, I'Anatomia e la Fisiologia, 3, 2, 301-316. (in Italian)

Canestrini, G. (1866): Prospetto critico dei pesci d'acqua dolce d'Italia. Archivio per la Zoologia, I'Anatomia e la Fisiologia, 4, 1, 47-187. (in Italian)

Canestrini, G. (1874): Fauna d'Italia. Parte terza. Pesci. Vallardi, Milano, 208pp. (in Italian)

Cano, J. M., Mäkinen, H. S., Leinonen, T., Freyhof, J., Merilä, J. (2008): Extreme neutral genetic and morphological divergence supports classification of Adriatic three-spined stickleback (Gasterosteus aculeatus): populations as distinct conservation units. Biological Conservation, 141, 1055-1066.

Car, L. (1900): Resultate einer naturwissenschaftlichen Studienraise. Glasnik Hrvatskoga naravoslovnoga društva, 1-3, 135-141. (in German)

Car, L. (1911): Biological classification and fauna of our fresh waters. Glasnik Hrvatskoga naravoslovnoga društva, 23, 1-2, 24-85. (in Croatian)

Carrara, F. (1846): La Dalmazia descritta. Fratelli Battana, Zadar, 192pp. (in Italian)

Carus, J. V. (1889-1893): Prodromus faunae Mediterraneae sive Descriptio animalium maris Mediterranei incolarum, quam comparata silva rerum quatenus innotuit, adiectis locis et nominibus vulgaribus eorumque auctoribus in commodum Zoologorum. Vol. II Brachiostomata, Mollusca, Cephalopoda, Tunicata, Vertebrata. E. Schweizerbart'sche Verlagshandlung, 
Stuttgart, 854pp. (in Latin)

Cigrovski Mustafić, M. (2012): Biochemical and histological profiles of species of genus Salmo in karstic rivers. PhD thesis. Facuty of Science, University of Zagreb.

Conci, C., Michelangeli, M. (1974): Catalogo dei tipi del Museo civico di storia naturale di Milano I. I tipi dei pesci, con note storiche sulla collezione ittologica. Atti della Societa Italiana di Scienze Naturali e del Museo Civili di storia naturale, 3-4, 213-237. (in Italian)

Crivelli, A. (1995): Are fish introductions a threat to endemic freshwater fishes in the northern Mediterranean region? Biological Conservation, 72, 311-319.

Crivelli, A. (1996): The freshwater fish endemic to the northern Mediterranean region - An action plan for their conservation. Station Biologique de la Tour du Valat, Arles, 171pp.

Crnić, V. (1923): Nase and its spawning. Lovačko-Ribarski vjesnik, 32, 7-11. (in Croatian)

Croatian State Archives (2016): State Archive Zagreb (HRHDA). Carsko Kraljevsko Ministarstvo Poljoprivrede (955). Dalmatinski spisi. 1909-1914, serija 4/b7 Va, kutija 126, mapa 69 i 1911-1917, serija 4/b7 J, kutija 119, mapa 28.

Csipkés, R., Szatmári, L., Soós, N. (2012): Nyugati pikó (Gasterosteus gymnurus) a Drávában. Halászat, 105, 1, 17-18. (in Hungarian)

Curić, Z. (1994): Protected nature in the lower Neretva area. Priroda, 84, 806, 17-20.

Čanadjija, S. (1977): The animal life in the Požega Valley and surrounding areas. In: Štrbašić M (ed), Požega 1227-1977. Skupština općine, Odbor za proslavu 750-godišnjice grada, Slavonska Požega, 66-71. (in Croatian)

Čanković, M., Kiškarolj, M., Kosorić, Đ. (1971): Investigation of infection of certain subspecies of Salmothymus obtusirostris Heck. with endohelminths in certain rivers of the Adriatic Basin. Ichthyologia, 3, 1, 13-18. (in Croatian)

Ćaleta, M. (2007): Ecological characteristics of the ichthyofauna of the Sava River lowlands. PhD thesis, Faculty of Science, University of Zagreb. (in Croatian with English abstract)

Ćaleta, M., Buj, I., Mrakovčić, M., Mustafić, P., Zanella, D., Marčić, Z., Duplić, A., Mihinjač, T., Katavić, I. (2015): Endemic Fish of Croatia. Agencija za zaštitu okoliša, Zagreb, 116pp. (in Croatian)

Ćaleta, M., Jelić, D., Buj, I., Zanella, D., Marčić, Z., Mustafić, P., Mrakovčić, M. (2011b): First record of the alien invasive species rotan (Perccottus glenii Dybowski, 1877) in Croatia. Journal of Applied Ichthyology, 27, 146-147.

Ćaleta, M., Mrakovčić, M., Buj, I., Mustafić, P., Zanella, D., Marčić, Z. (2009a): Threatened fishes of the world: Chondrostoma phoxinus Heckel, 1843 (Cyprinidae). Environmental Biology of Fishes, 84, 229-230.

Ćaleta, M., Tutman, P., Buj, I., Zanella, D., Mustafić, P.,
Marčić, Z., Mrakovčić, M., Dulčić, J. (2011a): How was Pirapitinga, Piaractus brachypomus (Serrasalmidae) introduced to Croatian freshwaters? Cybium, 35, 3, 259-261.

Ćaleta, M., Mrakovčić, M., Buj, I., Mustafić, P., Zanella, D., Marčić, Z. (2009b): Threatened fishes of the world: Aulopyge huegelli Heckel, 1842 (Cyprinidae). Environmental Biology of Fishes, 85, 21-22.

Ćurčić, V. (1910): National fisheries of Bosnia and Herzegovina. Glasnik Zemaljskog muzeja u Bosni i Hercegovini, 2-3, 379-488. (in Bosnian)

Ćurčić, V. (1915): Neretva and its trouts. Lovačko-Ribarski vjesnik, 24, 12-15. (in Bosnian)

Ćurčić, V. (1933): Enemies to fishes. Ribarski list, 8, 5-6, 62-64. (in Bosnian)

Ćurčić, V. (1938): Neretva and its trouts (Salmonidae). Ribarski list, 13, 7-8, 37-42. (in Bosnian)

Ćurčić, V. (1939a): Neretva and its trouts (Salmonidae). Ribarski list, 14, 1-2, 2-7. (in Bosnian)

Ćurčić, V. (1939b): Neretva and its trouts (Salmonidae). Ribarski list, 14, 5-6, 25-32. (in Bosnian)

Ćurčić, V. (1939c): Neretva and its trouts (Salmonidae). Ribarski list, 14, 7-8, 37-44. (in Bosnian)

Ćurčić, V. (1939d): Neretva and its trouts (Salmonidae). Ribarski list, 14, 9-10, 49-53. (in Bosnian)

Ćurčić, V. (1953): Trouts (Salmonidae) of Bosnia and Herzegovina). Ribarski list, 3, 35-39. (in Bosnian)

Damin, N. (1900): Zoological notes. Glasnik Hrvatskoga naravoslovnoga društva, 1-3, 148-149. (in Croatian)

Debeljak, Lj., Fašaić, K. (1985): Diet of the brown trout (Salmo trutta m. fario L.) in the Bajer Reservoir and Lepenica stream. Ichthyos, 1, 1-6. (in Croatian)

Debeljak, Lj., Fašaić, K., Bebek, Ž., Adamek, Z., Slačanec, D. (1993): Improving water quality in carp fishponds. Ribarstvo, 48, 3, 89-96. (in Croatian)

DeFaveri, J., Zanella, L. N., Zanella, D., Mrakovčić, M., Merilä, J. (2012): Phylogeography of isolated freshwater three-spined stickleback Gasterosteus aculeatus populations in the Adriatic Sea basin. Journal of Fish Biology, 80, 1, 61-85

Delić, A. (1984): Protection of the fish fauna in the open waters of the Novigrad municipality. Ribarstvo Jugoslavije, 39, 3-4, 52-58. (in Croatian)

Delić, A. (1985): Increases of length and weight of fish in the open waters of the Novigrad municipality. Ribarstvo Jugoslavije, 40, 6, 80-85. (in Croatian)

Delić, A. (1987): Diet of the black coot (Fulica atra L. 1758) at the Končanica fish farm. Ribarstvo Jugoslavije, 42, 2-3, 3-5. (in Croatian)

Delić, A. (1989): Ichthyofauna of the llova River in the upper llova area (central Croatia). Ribarstvo Jugoslavije, 44, 2, 26-28. (in Croatian)

Delić, A. (1993): Fish fauna of the watercourses of eastern Bilogora. Ribarstvo, 48, 2, 55-60. (in Croatian)

Delić, A., Bučar, M., Jugović, D., Mihoci, I., Kučinić, 
M. (2009): New data on the distribution of Cobitis elongatoides Bacescu \& Maier, 1869 in central Croatia with accompanying ichthyofauna. Natura Croatica, 18, 2, 255-262.

Delić, A., Bučar, M., Kučinić, M., Mihoci, I., Vilenica, M., Kiš-Novak, D., Dolenec, Z. (2014b): Contribution to the morphology of the Balkan loach, Cobitis elongata Heckel \& Kner, 1858 in Croatia. Natura Croatica, 23, 1, 15-25.

Delić, A., Bučar, M., Kučinić, M., Mrakovčić, M. (2003): New data about distribution of Sabanejewia balcanica (Karaman, 1922) (Cobitidae) in Croatia. Folia Biologica (Krakow), 51(Supplement), 39-42.

Delić, A., Grlica, I. D., Razlog-Grlica, J. (1997): New Umbra krameri Walbaum 1792 areas of distribution in Croatia. Ribarstvo, 55, 3, 93-98.

Delić, A., Kučinić, M., Bučar, M., Lazar, B., Mrakovčić, M. (2003): Morphometric and meristic characteristics of the goldside loach Sabanejewia balcanica (Cobitidae) in central Croatia. Folia Biologica (Krakow), 51(Supplement), 33-38.

Delić, A., Šanda, R., Bučar, M., Mihoci, I., Vilenica, M., Vukić, J., Lelo, S., Kučinić, M. (2014a): New data on distribution of the Monkey goby, Neogobius fluviatilis (Pallas, 1814) in Bosnia and Herzegovina and Croatia with notes on ecology and associated fish fauna. Natura Croatica, 23, 2, 298-302.

Delling, B. (2010): Diversity of western and southern Balkan trouts, with the description of a new species from the Louros River, Greece (Teleostei: Salmonidae). Ichthyological Exploration of Freshwaters, 21, 4, 331344.

Djikanović, V., Marković, G., Škorić, S. (2013): New record of Neogobius fluviatilis (Pallas, 1814) (Gobiidae) in the Danube River basin (Serbia). Archives of Biological Sciences, 65, 4, 1469-1472.

Dojmi, L. (1938): Combatting malaria biologically. Priroda, 28, 9, 268-278. (in Croatian)

Dojmi, L. (1939): Underground migration of the eels. Priroda, 29, 9, 263-269. (in Croatian)

Domitrović, Ž., Piria, M., Treer, T. (2004): Morphological parameters of five fish populations from the upper flow of the Sava River. Ribarstvo, 62, 3, 109-119. (in Croatian with English abstract)

Domitrović, Ž., Šprem, N., Treer, T., Piria, M. (2007): Growth and condition of carp from the Velo Blato Lake. In: Pospišil M (ed), Zbornik radova 42. hrvatskog i 2. međunarodnog simpozija agronoma. Zagreb, Croatia: University of Zagreb, 176-177. (in Croatian)

Dragun, Z., Filipović Marijić, V., Kapetanović, D., Valić, D., Vardić Smrzlić, I., Krasnići, N., Strižak, Ž., Kurtović, B., Teskeredžić, E., Raspor, B. (2013): Assessment of general condition of fish inhabiting a moderately contaminated aquatic environment. Environmental Science and Pollution Research, 20, 7, 4954-4968.

Dragun, Z., Filipović Marijić, V., Krasnići, N., Ivanković, D.,
Valić, D., Žunić, J., Kapetanović, D., Vardić Smrzlić, I., Redžović, Z., Grgić, I., Erk, M. (2018): Total and cytosolic concentrations of twenty metals/metalloids in the liver of brown trout Salmo trutta (Linnaeus, 1758) from the karstic Croatian river Krka. Ecotoxicology and Environmental Safety, 147, 537-549.

Dragun, Z., Krasnići, N., Strižak, Ž., Raspor, B. (2012): Lead concentration increase in the hepatic and gill soluble fractions of European chub (Squalius cephalus) an indicator of increased $\mathrm{Pb}$ exposure from the river water. Environmental Science and Pollution Research, 19, 6, 2088-2095.

Dragun, Z., Raspor, B., Podrug, M. (2007): The influence of the season and the biotic factors on the cytosolic metal concentrations in the gills of the European chub (Leuciscus cephalus L.). Chemosphere, 69, 6, 911-919.

Drnić, M. (1899): Fisheries of the Plitvice Lakes. Lovačkoribarski viestnik, 8, 7, 73-74. (in Croatian)

Dubac, M. (1961): Eels in wells. Priroda, 48, 8, 255-256. (in Croatian)

Dudan, M. I. (1891): On the breeding of carp. Šumarski list, 6, 263-274. (in Croatian)

Dudan, M. I. (1893): Fisheries and crustacean harvesting. Šumarski list, 7, 284-293. (in Croatian)

Dulčić, J., Dragičević, B., Ugarković, P., Tutman, P. (2017): The largemouth black bass (Micropterus salmoides) first record in the Neretva River delta, Adriatic drainage system of Croatia. Cybium, 41, 1, 77-78.

Dulčić, J., Glamuzina, B. (2006): Length-weight relationships for selected fish species from three eastern Adriatic estuarine systems (Croatia). Journal of Applied Ichthyology, 22, 254-256.

Dulčić, J., Glamuzina, B. (2010): Vulnerability assessments of the fisheries sector to climate change: case of the broader Neretva River delta. In: Fish and Fisheries of the Neretva River. In: Glamuzina, B., Dulčić, J. (eds), Ribe i Ribarstvo, rijeke Neretve. Sveučilište u Dubrovniku i Dubrovačko neretvanska županija, Dubrovnik, 3138pp. (in Croatian)

Dulčić, J., Tutman, P. (2015): Additional record of common bream Abramis brama (Cyprinidae) in the Adriatic drainage system (Norin River, Croatia). Annales, Series historia naturalis, 25, 2, 145-148.

Dulčić, J., Tutman, P., Matić-Skoko, S., Kraljević, M., JugDujaković, J., Glavić, N., Kožul, V., Glamuzina, B., Bartulović, V., Skaramuca, B. (2007): A list of Y-O-Y fish species found in the littoral shallows of the Neretva and Mala Neretva estuaries (Eastern Adriatic, Croatian coast). Acta Adriatica, 1, 89-94.

Dumbović, V., Posavec Vukelić, V., Duplić, A., Katušić, L., Jelić, D., Boršić, I., Partl A (2009): Study of the inventarisation of the flora and fauna of the Una River and its riparian belt. Sisačko-moslavačka županija, Sisak, 278pp. (in Croatian)

Duplić, A. (2008): Freshwater Fish. Manual for inventarisation and monitoring. State Institute for 
Nature Protection, Zagreb, 36pp.

Duplić, A. (2014): Taxonomy and biological characteristics of softmouth trout Salmo obtusirostris (Heckel, 1851) (Actinopterygii, Salmonidae). PhD thesis, Faculty of Science, University of Zagreb.

Duplić, A., Boršić, I. (2010): Invasive species in Croatia and in the Lonjsko Polje Nature Park. Lonjsko Polje Nature Park Bulletin, 11, 1-2, 7-22. (in Croatian)

Durand, J. D., Erhan, Ü., Doadrio, I., Pipoyan Templeton, S. R. (2000): Origin, radiation, dispersion and allopatric hybridization in the chub Leuciscus cephalus. Proceedings of the Royal Society, B 267, 1453, 16871697.

Dvoržak, R. (1897): Communications. Lovačko-ribarski viestnik, 6, 103. (in Croatian)

Dybowski, N. B. (1862): Versuch einer Monographie der Cyprinoiden livlands: nebst einer synoptischen Aufzählung der euopäischen Arten dieser Familie. Heinrich Laakmann, Dorpat, 215pp. (in German)

Economidis, P., Bănărescu, P. M. (1991): The distribution and origin of freshwater fishes in the Balkan Peninsula, Especially in Greece. Internationale Revue der gesamten Hydrobiologie und Hydrographie 76, 2, 257283.

Economidis, P., Šorić, V., Bănărescu, P. M. (2003): Barbus peloponnesius. In: Bănărescu, P. M., Bogutskaya, N. G. (eds), The Freshwater Fishes of Europe, Cyprinidae 2/II. Vol 5/II. AULA-Verlag, Wiebelsheim, 293-300.

EEA (European Environment Agency): (2002): Europe's biodiversity - biogeographical regions and seas. EEA Report No 1/2002.

Elvira, B. (1987): Taxonomic revision of the genus Chondrostoma Agasiz, 1835 (Pisces, Cyprinidae). Cybium, 11, 2, 111-140.

Elvira, B. (1997): Taxonomy of the genus Chondrostoma (Osteichthyes, Cyprinidae) an updated reviev. Folia Zoologica, 46, 1, 1-14.

Eros, T., Sallai, Z., Kotusz, J. (2003): Distribution and conservation status of loaches in Hungary. Folia Biologica (Krakow), 51(Supplement), 17-19.

Eschmeyer, W. N., Fricke, R., van der Laan, R. (eds) (2018a): Catalog of fishes: classification (http:// www.calacademy.org/scientists/catalog-of-fishesclassification/). Electronic version accessed 01. 10. 2018.

Eschmeyer, W. N., Fricke R, van der Laan, R. (eds) (2018b): Catalog of fishes: genera, species, references (http://researcharchive.calacademy.org/research/ ichthyology/catalog/fishcatmain.asp). Electronic version accessed 01. 10. 2018.

Esteve, M., McLennan, D. A., Zablocki, J. A , Pustovrh, G. , Doadrio, I. (2014): Spawning behaviour and the softmouth trout dilemma. Archives of Polish Fisheries, 22, 2, 159-165.

Faber, G. L. (1883): Fisheries of the Adriatic and the fish thereof: A Report of the Austro-Hungarian sea fisheries, with a detailed description of the Marine Fauna of the Adriatic Gulf. Quuaritch, London, 25-39.

Farkaš-Vukotinović, L. (1858): Die Plitvica-Seen in der oberen Militärgrenze in Kroatien. Sitzungsberichte der Kaiserlichen Akademie der Wissenschaften. Mathematisch-Naturwissenschaftliche Classe, 26, 268276. (in German)

Fašaić, K., Mrakovčić, M., Mišetić, S. (1990): Water chemistry and ichthyoproduction of Visovac Lake. In: Kerovec, M. (ed), Stanje istraženosi i problemi zaštite ekosistema, Knjiga 2, Hrvatsko ekološko društvo, Zagreb, 365-375. (in Croatian)

Fijan, N. (1948a): Transport and stocking of carp in Vrana Lake. Ribarstvo Jugoslavije, 2, 6, 45-46. (in Croatian)

Fijan, N. (1948b): Moving of Vrana Lake under the competence of the Ministry of Fisheries of the People's Republic of Croatia. Ribarstvo Jugoslavije, 2, 6, 61. (in Croatian)

Fijan, N. (1949): Anniversary of the stocking of carp in Vrana Lake. Ribarstvo Jugoslavije, 3, 5, 45-46. (in Croatian)

Fijan, N. (1950): A secondary predator fish for the best exploitation of fish ponds - largemouth bass. Slatkovodno Ribarstvo Jugoslavije, 4, 8, 163-164. (in Croatian)

Fijan, N. (1951a): Three years of breeding carp in Vrana Lake. Slatkovodno Ribarstvo Jugoslavije, 5, 3, 46-52. (in Croatian)

Fijan, N. (1951b): Carp stocking in the area of Neretva Blato. Slatkovodno Ribarstvo Jugoslavije, 5, 1-2, 9-12. (in Croatian)

Fijan, N. (1953): Fish catch on Vrana Lake in 1952. Ribarstvo Jugoslavije, 8, 1, 23. (in Croatian)

Fijan, N. (1956): Stocking Vrana Lake with freshwater fish. Morsko Ribarstvo, 8, 2, 54-56. (in Croatian)

Fijan, N. (1964): New fish farming at Siščani near Čazma. Ribarstvo Jugoslavije, 19, 3, 77-80. (in Croatian)

Fijan, N. (1975): Memoirs of an official trip to Vrana Lake. Ribarstvo Jugoslavije, 30, 6, 140-141. (in Croatian)

Fijan, N. (1976a): First artificial spawning of catfish. Ribarstvo Jugoslavije, 31, 6, 134-136. (in Croatian)

Fijan, N. (1976b): The issues of Trakošćan Lake and the small fish farms in the Varaždin area. Ribarstvo Jugoslavije, 31, 5, 112-114. (in Croatian)

Fijan, N., Petrinec, Z., Đorđević, V. (1989): Taxonomy and production of the North American bullheads of the genus Ictalurus. Ribarstvo Jugoslavije, 44, 5, 109-113. (in Croatian)

Fijan, N., Vojta, J. (1969): First spawning of the silver/ bighead carp and grass carp at the Končanica fish farm. Ribarstvo Jugoslavije, 24, 6, 124-125. (in Croatian)

Filipović Marijić, V., Raspor, B. (2014): Relevance of biotic parameters in the assessment of the spatial distribution of gastrointestinal metal and protein levels during spawning period of European chub (Squalius cephalus L.). Environmental Science and Pollution Research, 21, 
12, 7596-7606.

Filipović Marijić, V., Vardić Smrzlić, I., Raspor, B. (2013): Effect of acanthocephalan infection on metal, total protein and metallothionein concentrations in European chub from a Sava River section with low metal contamination. Science of the Total Environment, 463-464, 772-780.

Filipović Marijić, V., Vardić Smrzlić, I., Raspor, B. (2014): Does fish reproduction and metabolic activity influence metal levels in fish intestinal parasites, acanthocephalans, during fish spawning and postspawning period? Chemosphere, 112, 449-455.

Fink, N. (1920): On route to - Atlantis. Priroda, 10, 10, 201204. (in Croatian)

Fink, N. (1947): Shortnose sturgeon. Priroda, 34, 2, 42-45. (in Croatian)

Fink, V. (1932a): A summer on the Drava. Ribarski list, 7, 3-4, 39-41. (in Croatian)

Fink, V. (1932b): A summer on the Drava. Ribarski list, 7, 5-6, 58-60. (in Croatian)

Fitzinger, L. J., Heckel, J. J. (1836): Monographische Darstellung der Gattung Acipenser. Annalen des Wiener Museums der Naturgeschichte, 1, 261-326. (in German)

Flögel, R. (1894): Communications. Lovačko-ribarski viestnik, 3, 56-57. (in Croatian)

Fodolović, S. (1973): Yet another trophy. Ribarski list, 3, 78. (in Croatian)

Fortis, A. (1771): Saggio d'osservazioni sopra l'isola di Cherso ed Osero. Società Imperiale, e Reale di Siena, ec. in Venezia, 132pp.

Fortis, A. (1774): Viaggio in Dalmazia-volume primo. Alvise Milocco, Venezia, 204pp. (in Italian)

Franičević, M., Tičina, V. (2003): Biometric characteristics of a rare endemic fish, Phoxinellus adspersus (Heckel 1843) from Red Lake (Imotski, Croatia). Periodicum Biologorum, 105, 4, 453-460.

Franić, D. (1898): Invitation for the collection of hydrographic data. Gospić. (in Croatian)

Franić, D. (1900): Twice over Velebit from Velebit to Stari Grad. Hrvatski planinar, 3, 41-44. (in Croatian)

Franić, D. (1910): Plitvice Lakes and the environs. Kraljevska zemaljska tiskara, Zagreb, 439pp. (in Croatian)

Franić, R. (1949): The pike are spawning.... Ribarstvo Jugoslavije, 4, 6, 67. (in Croatian)

Franke, J. (1892): Die Gewässer in Krain und ihre nutzbare Fauna. Jahresbericht der k. k. Oberrealschule in Laibach, 1-24. (in German)

Freyhof, J. (1997): Zur Fortpflanzungsbiologie des Barbengründlings. Die Aquarien und Terrarien Zeitschrift, 50, 513-516. (in German)

Freyhof, J. (2011): Diversity and distribution of freshwater gobies from the Mediterranean, the Black and Caspian Seas, pp. 279-288. In: Patzner RA, Van Tassell JL, Kovačić, M., Kapoor BG (eds), The Biology of Gobies.
Science Publishers, Enfield, New Hampshire, 652pp.

Freyhof, J. (2012): Threatened freshwater fishes and molluscs of the Balkan, potential impacts of hydropower projects. Unpublished report, ECA Watch Austria \& EuroNatur, 81pp.

Freyhof, J., Lieckfeldt, D., Pitra, C., Ludwig, A. (2005a): Molecules and morphology: Evidence for introgression of mitochondrial DNA in Dalmatian cyprinids. Molecular Phylogenetics and Evolution, 37, 347-354.

Freyhof, J., Kottelat, M., Nolte, A. (2005b): Taxonomic diversity of European Cottus with description of eight new species (Teleostei: Cottidae). Ichthyological Exploration of Freshwaters, 16, 2, 107-172.

Freyhof, J., Lieckfeldt, D., Bogutskaya, N. G., Pitra C, Ludwig A (2006): Phylogenetic position of the Dalmatian genus Phoxinellus and description of the newly proposed genus Delminichthys (Teleostei: Cyprinidae). Molecular Phylogenetics and Evolution, 38, 416-425.

Freyhof, J., Stelbrink, B. (2007): Cobitis illyrica, a new species of loach from Croatia (Teleostei: Cobitidae). Ichthyological Exploration of Freshwaters, 18, 3, 269275.

Frick, W. (1883): Die Herrschaft Béllye ein, Ungarischer Grossgrundbesitz Sr. Kaiserl. Hoheit des Erzherzogs Alberecht. Ungarischen Landes-Agricultur Verein in Budapest, Wien, 312pp. (in German)

Friedrich, T., Wiesner, C., Zangl, L., Daill, D., Freyhof, J., Koblmüller, S. (2018): Romanogobio skywalkeri, a new gudgeon (Teleostei: Gobionidae) from the upper Mur River, Austria. Zootaxa, 4403, 2, 336-350.

Froese, R., Pauly, D. (eds) 2019. FishBase. World Wide Web electronic publication. www.fishbase.org, version (04/2019).

Gabelica, I., Piasevoli, G., Mekinić, S. (2011): Prološko Blato Significant Landscape. Hrvatska vodoprivreda, 194, 84-87. (in Croatian)

Gandolfi-Hornyold, A. (1933): Eta e accrescimento delle piccole anguille del porto di Rovigno Osservazioni sopra alcune anguille del lago di Boccagnazzo presso Zara e nel lago d'Arsa in Istria. Note dell'istituto ItaloGermanico di biologia marina di Rovigno d'Istria, 7, 1-29. (in Italian)

Gandolfi-Hornyold, A. (1938): Observations sur l'anguille de la cote Yugoslave de I'Adriatique (Neretva, Split et Trogir). Godišnjak Oceanografskog instituta Kraljevine Jugoslavije, 1, 159-177. (in French)

Geiger, M. F., Herder, F., Monaghan, M. T., Almada, V., Barbieri, R., Bariche, M., Berrebi, P., Bohlen, J., CasalLopez, M., Delmastro, G. B., Denys, G. P. J., Dettai, A., Doadrio, I., Kalogianni, E., Kärst, H., Kottelat, M., Kovačić, M., Laporte, M., Lorenzoni, M., Marčić, Z., Özuluğ, M., Perdices, A., Perea, S., Persat, H., Porcelotti, S., Puzzi, C., Robalo, J., Šanda, R., Schneider, M., Šlechtova, V., Stoumboudi, M., Walter, S., Freyhof, J. (2014): Spatial heterogeneity in the Mediterranean Biodiversity Hotspot affects barcoding accuracy of its 
freshwater fishes. Molecular Ecology Resources, 14, 6, 1210-1221.

Georgiev, S. (2003): On the origin of the Balkan peninsula salmonids. Ribarstvo, 61, 4, 147-174.

Georgiev, S. (2011): Study of Salmo genus (Pisces, Salmonidae): on the Balkan peninsula. Ribarstvo, 69, 1, 11-20.

Getz, D. (2004): The Middle Ages in Baranja; a refernce to landscape, forestry and fishing. Šumarski list, 1-2, 4154. (in Croatian with English abstract)

Giglioli, E. H. (1880): Elenco dei mammiferi, degli uccelli e dei rettili ittiofagi appartenenti alla Fauna Italica o catalogo degli anfibi e dei pesci Italiani. Firenze, 55pp. (in Italian)

Gioseffi, M. (1926): Le „Gambusie“ nella lotta antimalarica in Istria. Rivista di Malariologia, 5, 4, 469-475. (in Italian)

Giunio, P. (1970): From the Neretva to the ocean depths. Morsko Ribarstvo, 22, 4, 177-182. (in Croatian)

Glamuzina, B. (2010): Neretva fisheries: history and perspectives. In: Glamuzina, B., Dulčić, J. (eds), Ribe i Ribarstvo, rijeke Neretve. Sveučilište u Dubrovniku i Dubrovačko neretvanska županija, Dubrovnik, 2030pp. (in Croatian)

Glamuzina, B., Bartulović, V. (2010): Grey mullets of Neretva River estuary: biological and economical characteristics. In: Glamuzina, B., Dulčić, J. (eds), Ribe i Ribarstvo, rijeke Neretve. Sveučilište u Dubrovniku i Dubrovačko neretvanska županija, Dubrovnik, 92114pp. (in Croatian)

Glamuzina, B., Bartulović, V., Dulčić, J., Conides, J. A., Tutman, P., Matić-Skoko, S., Gavrilović, J., Jug-Dujaković, J., Hasković, E., Ivanc, A., Zovko, N. (2007): Some biological characteristics of the endemic Neretvan nase, Chondrostoma knerii Heckel, 1843, in the Hutovo Blato wetlands (Bosnia and Herzegovina). Journal of Applied Ichthyology, 23, 221-225.

Glamuzina, B., Bogut, I., Ivančević, M. (2011): Revitalisation of the indigenous and endemic Neretva trouts. Federalni agromediteranski zavod, Mostar, 30pp. (in Croatian)

Glamuzina, B., Dulčić, J., Tutman, P., Matić-Skoko, S., Tomšić, S., Bartulović, V. (2009): Record size of Salmo dentex (Heckel, 1851): (Salmonidae): from Neretva River (Bosnia and Herzegovina). Cybium, 33, 2, 165167.

Glamuzina, B., Pavličević, J., Bogut, I., Snoj, A. (2010a): The endemic Neretva trout - an issue of systematics. 1. međunarodni simpozij ribarstva i ribolovnog turizma. Centar za Ribarstvo, Konjic, 29-38. (in Croatian)

Glamuzina, B., Pavličević, J., Tutman, P., Glamuzina, L., Bogut, I., Dulčić, J. (2013): Fishes of Neretva. Udruga CEAV, Mostar; Modrozelena - Zadruga branitelja, Metković, 263pp. (in Croatian)

Glamuzina, B., Tutman, P., Pavličević, J., Bogut, I., Dulčić, J. (2010b): Biodiversity of the fishes of Herzegovina.
Međunarodni kolokvij „2010. godina bioraznolikosti“, Federalno ministarstvo okoliša i turizma, Sarajevo, 1-11. (in Croatian)

Glavan, I. (1949): Wood shavings in the Dretulja. Ribarstvo Jugoslavije, 4, 6, 91. (in Croatian)

Glavan, I. (1952): It was in January. Ribarstvo Jugoslavije, 7, 7, 126-127. (in Croatian)

Glowacki, J. (1885): Die Fische der Drau und ihres Gebietes. Wissenschaftliche Abhandlungen Jahresbericht des Steiermärkischen landschaft Unter-Gymnasiums zu Pettau, 16, 95, 1-18. (in German)

Glowacki, J. (1896): Die Fischfauna der Save und der Isonzo. I. Jahresbericht d. k. k. Staats-Untergymnasiums in Chilli 1895-1896, 1-37. (in German)

Golub, S. (2011): Mura-Drava Regional Park: management challenges. Hrvatska vodoprivreda 197, 74-79. (in Croatian)

Grce, Z. (1956): Fisheries of Vrana Lake. Morsko Ribarstvo, 8, 7, 217-218. (in Croatian)

Grce, Z. (1957): On the results of fisherman from Kopačevo in Vrana Lake. Morsko Ribarstvo, 9, 10, 266-267. (in Croatian)

Grce, Z. (1959): Fish species in Vrana Lake. Morsko Ribarstvo, 11, 1, 9.

Gridelli, E. (1936): I Pesci d`Acqua Dolce della Venezia Giulia. Del Bianco, Udine, 152pp. (in Italian)

Grisogono, P. N. (1780): Notizie per servire alla storia naturale della Dalmazia. Giulio Trento, Trevigi, 190pp. (in Italian)

Grloci (1926): Two trout fishing. Lovačko-Ribarski vjesnik, 35, 332-334. (in Croatian)

Grmek, M., Balabanić, J. (2000): On the fishes and bivalves of the Dubrovnik region. Correspondence of Sorkočević-Aldrovandi, Dubrovnik-Bologna: 15801584. Dom i svijet, Zagreb, 193pp. (in Croatian)

Grubišić, F. (1957): Extraction of carp in Vrana Lake. Morsko Ribarstvo, 9, 5, 144-145.

Guti, G. (2006): Past and present status of sturgeons in Hungary. Proceedings of 36th International Conference of IAD. Austrian Committee Danube Research/IAD, Vienna, 143-147.

Guti, G., Gaebele, T. (2009): Long-term changes of sterlet (Acipenser ruthenus) population in the Hungarian section of the Danube. Opuscula Zoologica Budapest, $40,2,17-25$.

Guzina, N., Vuković, T. (1971): Microelements iron and copper in liver and muscle tissue of certain endemic species of the genus Paraphoxinus. Ichthyologia, 3, 1, 19-25. (in Bosnian)

Guzina, N. (2000): Morphological and taxonomic characteristics of the species Aulopyge hügeli Heckel, 1841. PhD thesis, Faculty of Science, University of Sarajevo. (in Bosnian)

Habeković, D. (1967): Exterior of the carp of Vrana Lake. Ribarstvo Jugoslavije, 22, 4, 96-104. (in Croatian) 
Habeković, D. (1973): Exterior of the carp of Vrana Lake. Izbor naučnih i stručnih radova Instituta za slatkovodno Ribarstvo, SRH, Zagreb, 93-105. (in Croatian)

Habeković, D. (1978a): Introduction of the whitefish into our waters. Ribarstvo Jugoslavije, 33, 6, 143-144. (in Croatian)

Habeković, D. (1978b): First results of cage breeding of rainbow trout in lake conditions. Ribarstvo Jugoslavije, 33, 3, 52-64. (in Croatian)

Habeković, D. (1982): Cage breeding of rainbow trout. Ribarstvo Jugoslavije, 37, 1, 4-8. (in Croatian)

Habeković, D. (1987): Haematology properties of catfish (Silurus glanis L.). Ichthyologia, 19, 1, 77-87. (in Croatian)

Habeković, D. (1991): The hematologic characteristics of sexual dimorphism of tench (Tinca tinca L.): during spawning period. Ichthyologia, 23, 1, 17-24.

Habeković, D. (1993): Ichthyofauna of the accumulation lake Prančevići on the River Cetina. Ribarstvo, 48, 4, 125-135. (in Croatian with English abstract)

Habeković, D. (1994a): Ichthyofauna of the accumulation lake Prančevići on the River Cetina. III Cyprinidae. Ribarstvo, 52, 4, 163-171. (in Croatian with English abstract)

Habeković, D. (1994b): Ichthyofauna of the accumulation lake Prančevići on the River Cetina. II Salmonide. Ribarstvo, 52, 2, 59-64. (in Croatian with English abstract)

Habeković, D., Aničić, I., Safner, R. (1993): Growth dynamics of the chub in the River Sava. Ribarstvo, 48, 3, 79-88. (in Croatian with English abstract)

Habeković, D., Čolo-Ančić, I. (1986): Success of stocking Peruća Lake with whitefish. Saopštenje s XIII. kongresa Jugoslavenskog društva za visoke brane, Mostar, knjiga 1, pp. 209-212. (in Croatian)

Habeković, D., Homen, Z., Fašaić, K. (1990): Ichthyofauna of part of the Sava River. Ribarstvo Jugoslavije, 45, 1-2, 8-14. (in Croatian with English abstract)

Habeković, D., Mišetić, S. (2000): Impacts of the hydro reservoir on the fish community in the Cetina River from Peruča to Prančević. In: Kerovec, M., Durbešić, P. (eds), Natural history research of the Biokovo area. Hrvatsko ekološko društvo, Zagreb, 183-193. (in Croatian)

Habeković, D., Mišetić, S., Marko, S. (1981): Cage breeding of Coregonus peled. Ribarstvo Jugoslavije, 36, 6, 121127. (in Croatian)

Habeković, D., Mrakovčić, M., Bogdan, M. (1986): Ichthyofauna of part fo the Drava River after the construction of the Čakovec hydroelectric system. Ribarstvo Jugoslavije, 41, 4-5, 57-61. (in Croatian)

Habeković, D., Pažur, K. (1998): Pike (Esox lucius L.) - its characteristics and importance. Ribarstvo, 56, 2, 55-60. (in Croatian with English abstract)

Habeković, D., Pažur, K. (2000): Ichthyofauna of some of the waters of the Imotski area and ways to improve fisheries. In: Kerovec, M., Durbešić, P. (eds),
Prirodoslovna istraživanja biokovskog područja. Hrvatsko ekološko društvo, Zagreb, 195-202. (in Croatian)

Habeković, D., Pažur, K., Popović, J. (1992): Ichthyofauna of some running waters of Lika. Ribarstvo, 47, 1-2, 2333. (in Croatian with English abstract)

Habeković, D., Popović, J. (1991): State and exploitation of the fish fund in the River Sava from Podsused to Strelečko. Ribarstvo Jugoslavije, 46, 1-2, 1-9. (in Croatian with English abstract)

Habeković, D., Safner, R., Aničić, I., Treer, T. (1997): Ichthyofauna of a part of the River Sava. Ribarstvo, 55, 3, 99-110. (in Croatian with English abstract)

Hadžić, F. (1964a): My impressions of the Bosut. Ribarski list, 2, 42. (in Bosnian)

Hadžić, F. (1964b): Declining numbers of catfish in the Sava and its tributaries. Ribarski list, 4, 81. (in Bosnian) Hadžić, F. (1964c): „Blooming“ of the Sava. Ribarski list, 4, 86, (in Bosnian)

Hadžić, F. (1965): Our records (from the Sava). Ribarski list, 5, 108. (in Bosnian)

Hadžić, F. (1967): Surprise from the Sava River. Ribarski list, 3, 90. (in Bosnian)

Hafner-Lahorski, R. (1935a): Trout in the Slavonian streams. Ribarski vjesnik, 13, 9-10, 234-238. (in Croatian)

Hafner-Lahorski, R. (1935b): Summertime in October under the Krka waterfalls. Ribarski vjesnik, 13, 9-10, 275-280. (in Croatian)

Hafner-Lahorski, R. (1935c): Fishing tourism tour by a Slavonian angler to the trout waters of Bosnia, Herzegovina, Dalmatia, Slovenia and Gorski Kotar. Ribarski vjesnik, 13, 12, 293-298. (in Croatian)

Hafner-Lahorski, R. (1947a): Lake trout in our waters. Ribarstvo Jugoslavije, 2, 5, 79. (in Croatian)

Hafner-Lahorski, R. (1947b): Our giant trout. Ribarstvo Jugoslavije, 5, 79-80. (in Croatian)

Hafner-Lahorski, R. (1947c): The huchens of Siberia and the Far East. Ribarstvo Jugoslavije, 2, 5, 97-99. (in Croatian)

Hafner-Lahorski, R. (1948a): On the protection of the huchen. Ribarstvo Jugoslavije, 3, 6, 69-70. (in Croatian)

Hafner-Lahorski, R. (1948b): Heavy barbel caught. Ribarstvo Jugoslavije, 3, 11-12, 121. (in Croatian)

Hafner-Lahorski, R. (1950a): Rare pike-perch catch. Slatkovodno Ribarstvo Jugoslavije, 5 3-4, 60. (in Croatian)

Hafner-Lahorski, R. (1950b): A letter from Osijek. Slatkovodno Ribarstvo Jugoslavije, 5, 5, 80-81. (in Croatian)

Hafner-Lahorski, R. (1950c): A sterlet with live young in the body. Slatkovodno Ribarstvo Jugoslavije, 5, 5, 91. (in Croatian)

Hafner-Lahorski, R. (1953a): Restoration of the trout farm at Mt. Papuk. Ribarstvo Jugoslavije, 8, 1, 17-18. (in 
Croatian)

Hafner-Lahorski, R. (1953b): Eel caught in the Drava River. Ribarstvo Jugoslavije, 8, 1, 22. (in Croatian)

Hamann, O. (1896): Europäische Höhlenfauna: Eine Darstellung der in den Höhlen Europas lebenden Tierwelt mit besonderer Berücksichtigung der Höhlenfauna Krains. Nach eigenen Untersuchungen. Hermann, Jena, 296pp. (in German)

Hardisty, M. W. (1986): Petromyzon marinus Linnaeus, 1758. In: Holčík, J. (ed), The Freshwater Fishes of Europe. Petromizontiformes 1, AULA-Verlag, Wiesbaden, 94-116.

Harka, Á. (1992): A Dráva halai. Halászat 85, 1, 9-12. (in Hungarian)

Hasenay, M. (1933): Farming Largemouth bass at Belje. Ribarski list, 8, 3-4, 52. (in Croatian)

Has-Schön, E., Bogut, I., Strelec, I. (2006): Heavy metal profile in five fish species included in human diet, Domiciled in the end flow of River Neretva (Croatia). Archives of Environmental Contamination and Toxicology, 50, 545-551.

Hawlitschek, A. (1895): Die Salmen der österreichischen Gewässer. Mittheilungen des Österreichischen Fischerei-Vereines, 15, 57, 27-34. (in German)

Hawlitschek, A. (1898): Der Sterlet - Acipenser Ruthenus. Mittheilungen des Österreichischen Fischerei-Vereines, 18, 9, 168-175. (in German)

Heckel, J. J. (1841): On a new European genus of freshwater fish. The Annals and Magazine of Natural History: Zoology, Botany, and Geology, 7, 46, 522-523.

Heckel, J. J. (1841): Ueber eine neue Gattung (Genus) von Süsswasserfischen in Europa. Bulletin scientifique publié par l'Académie impériale des sciences de SaintPétersbourg 8, 384. (in German)

Heckel, J. J. (1842): Beschreibung eines in Dalmatien im Herbst 1840 entdeckten neuen Fisches. In: Strombeck, F. K., Mansfeld, D. (eds), Amtlicher Bericht über die neunzehnte Versammlung deutscher Naturforscher und Aerzte zu Braunschweig im September 1841. Braunschweig Friedrich Vieweg \& Sohn, Wiesbaen, 7374. (in German)

Heckel, J. J. (1843): Ichthyologie (von Syrien). In: von Russegger J (ed), Reisen in Europa, Asien und Afrika, mit besonderer Rücksicht auf die naturwissenschaftlichen Verhältnisse der betreffenden Länder unternommen in den Jahren 1835 bis 1841, etc. E. Schweizerbart'sche Verlagshandlung, Stuttgart, 991-1099. (in German)

Heckel, J. J. (1852): Beiträge zu den Gattungen Salmo, Fario, Salar, Coregonus, Chondrostoma und Telestes. Sitzungsberichte der Kaiserlichen Akademie der Wissenschaften in Wien, MathematischNaturwissenschaftlichen Classe 8, 347-390. (in German)

Heckel, J. J., Kner, R. (1858): Die Süsswasserfische der Österreichischen Monarchie, mit Rücksicht auf die angränzenden Länder, Leipzig, 388pp. (in German)
Hefele, F. (1889): A little on the fish based on the observations of people surrounding Sisak. Glasnik Hrvatskoga naravoslovnoga družtva, 1-5, 72-78. (in Croatian)

Heintz, K. (1915): Trout (Salmonidae) of Bosnia and Herzegovina. Lovačko-Ribarski vjesnik, 24, 55-59. (in Croatian)

Hensel, K., Holčík, J. (1997): Past and current status of sturgeons in the upper and middle Danube River. Environmental Biology of Fishes, 48, 185-200.

Herga, V. (1968): Slow death of a large river. Ribarski list, 4, 115. (in Croatian)

Herga, V. (1974): Fish, of the fish, to the fish.... Ribarski list, 4, 16-24. (in Croatian)

Hietzinger, K. B. E. (1817): Producte des Thierreiches. In: Gerold C (ed), Statistik der Militärgrenze des oesterreichischen Kaiserthums, ein Versuch, Erster Theil, Carl Gerold, Wien, 150-160. (in German)

Hirc, D. (1891): Croatian Coastal Area. Hartman, Zagreb, 301pp. (in Croatian)

Hirc, D. (1896): How is the fishing on the Danube and other waters?. Lovačko-ribarski viestnik, 5, 124-127. (in Croatian)

Hirc, D. (1897): Material for the national nomenclature and terminology of animals. Folk names of fishes. Nastavni vjesnik 5, 160-174. (in Croatian)

Hirc, D. (1898): Gorski Kotar. Tiskara Rijeka, Rijeka, $175 p p$. (in Croatian)

Hirc, D. (1899a): A look into the underground world of our homeland. Hrvatski planinar, 3, 36-39. (in Croatian)

Hirc, D. (1899b): A look into the underground world of our homeland, part II. Hrvatski planinar, 4, 56-60. (in Croatian)

Hirc, D. (1899c): In Gorski Kotar. Hrvatski planinar, 10, 145148. (in Croatian)

Hirc, D. (1899d): Some fishes make sounds. Lovačkoribarski viestnik, 8, 128-130. (in Croatian)

Hirc, D. (1899e): The fish fins. Lovačko-ribarski viestnik, 8, 132-133. (in Croatian)

Hirc, D. (1900a): Trout - a contribution ot the ichthyofauna of Croatia and Slavonia. Lovačko-ribarski viestnik, 9, 7072. (in Croatian)

Hirc, D. (1900b): Lika and the Plitvice Lakes: memoirs of a journey. Hartman, Zagreb, 163 pp. (in Croatian)

Hirc, D. (1902a): Natural history tales - Rhodeus amarus. Glasnik Hrvatskoga naravoslovnoga društva, 4-6, 166. (in Croatian)

Hirc, D. (1902b): Natural history tales - flora of stinking hot springs. Glasnik Hrvatskoga naravoslovnoga društva, 4-6, 166-167. (in Croatian)

Hirc, D. (1902c): Huchen and „Lachsforelle“. Glasnik Hrvatskoga naravoslovnoga društva, 4-6, 455-457. (in Croatian)

Hirc, D. (1902d): Surrounding Marijanac. Hrvatski planinar, 11-12, 83-89. (in Croatian) 
Hirc, D. (1911): Contributions to the fauna and flora of Kalnička Gora. Glasnik Hrvatskoga naravoslovnoga društva, 23, 3, 46-53. (in Croatian)

Hirc, D. (1925): Lika and Gacka. Hrvatski planinar, 21, 169172. (in Croatian)

Hirtz, M. (1928): Are there eels in the Danube and its tributaries?. Priroda, 18, 1, 30. (in Croatian)

Hirtz, M. (1937): A massive huchen. Priroda, 27, 3, 93-94.

Holčík, J. (1999): Rhodeus sericeus (Pallas, 1776). In: Bănărescu, P. M. (ed), The Freshwater Fishes of Europe. Cyprinidae 2/I. Vol. 5/I. AULA-Verlag, Wiebelsheim, 2-32.

Holčík, J., Delić, A. (2000): New discovery of the Ukrainian brook lamprey in Croatia. Journal of Fish Biology, 56, 73-86.

Holčik J, Delić, A., Kučinić, M., Bukvić V, Vater, M. (2004): Distribution and morphology of the sea lamprey from the Balkan coast of the Adriatic Sea. Journal of Fish Biology, 64, 514-527.

Holčík, J., Kinzelbach, R., Sokolov, L. I., Vasil'ev, V. P. (1989): Acipenser sturio Linnaeus, 1758. In: Holčík, J. (ed), The Freshwater Fishes of Europe. Vol 1, Part II, General introduction to fishes, Acipenseriformes. AULA-Verlag, Wiesbaden, 367-394.

Holčík, J., Mrakovčić, M. (1997): First record of Lethenteron zanandreai (Cyclostomata, Petromyzontidae) in the Adriatic basin of the Balkan peninsula and its zoogeographic consequences. Folia Zoologica, 46, 263271.

Homen, Z., Fašaić, K. (1981): Growth dynamics of the brown trout (Salmo trutta m. fario L. 1758) in the Zrmanja River and its tributaries. Ribarstvo Jugoslavije, 36, 3, 57-61. (in Croatian)

Homen, Z., Mikuška, J., Radanović, P., Mikuška, Z. (1991): Qualitative and quantitative components of the fish fund of the River Danube and flooded region in Kopački Rit. Ribarstvo Jugoslavije, 46, 1-2, 27-30. (in Croatian with English abstract)

Honsig-Erlenburg, W., Konar, M., Huber, T., Gutleb, B., Wieser, G., Friedl, T., Mildner, P. (1997): Zoologische Exkursion des Naturwissenschaftlichen Vereines zur Kolpa (Slowenien). Carinthia, II 187, 107, 139-152. (in German)

Horvat, M. (2005): Brown trout (Salmo trutta f. fario). Ribolov, 63, 42-45. (in Croatian)

Horvat, R. (1941): Lika and Krbava Rivers: historical notes. Matica hrvatska, Zagreb, pp182. (in Croatian)

Horvat, Š. (1900a): A little about our fisheries and fish farming. Lovačko-ribarski viestnik, 9, 83-84. (in Croatian)

Horvat, Š. (1900b): A little about our fisheries and fish farming. Lovačko-ribarski viestnik, 9, 95-97. (in Croatian)

Horvat, Š. (1900c): A little about our fisheries and fish farming. Lovačko-ribarski viestnik, 9, 118-119. (in Croatian)
Horvat, Š. (1900d): A little about our fisheries and fish farming. Lovačko-ribarski viestnik, 9, 139-142. (in Croatian)

Horvat, Š. (1901): Fish and fish farming. Društvo sv. Jeronima, Zagreb, 164pp. (in Croatian)

Host, J. (1993): Viaggio botanico nell'Istria, Isole del Quarnero, e nella Dalmazia, incominciato il dì 14 d'Agosto 1801. e terminato il dì 6 d'Agosto 1802.). Transkripcija i prijevod: Krešimir Čvrljak, Matica hrvatska, Rijeka, 78-79.

Houška, J. (1924): Eel. Ribarski vjesnik, 2, 9-10, 37-38. (in Croatian)

Howes, G. J. (1985): A revised synonymy of the minnow genus Phoxinus Rafinesque, 1820 Teleostei Cyprinidae with comments on its relationships and distribution. Bulletin of the British Museum (Natural History) Zoology, 48, 57-74

Howes, G. J. (1987): Phylogenetic position of the Yugoslavian cyprinid fish genus Aulopyge Heckel, 1841, with an appraisal of the genus Barbus Cuvier and Cloquet, 1816 and the subfamily Cyprininae. Bulletin of the British Museum Natural History (Zoology), 52, 165-196.

Hrbek, T., Stölting, K. N., Bardakci, F., Küçük, F., Wildekamp, R. H., Meyer, A. (2004): Plate tectonics and biogeographical patterns of the Pseudophoxinus (Pisces: Cypriniformes) species complex of central Anatolia, Turkey. Molecular Phylogenetics and Evolution, 32, 297-308.

Hruška, V. (1911): Successful fishing at Zalesina (near Delnice). Lovačko-Ribarski vjesnik, 20, 23. (in Croatian)

Hrženjak, T., Ehrlich, I. (1981): Lipids in the salmonids in the waters of Plitvice Lakes National Park. Ekologija, 2, 133-140. (in Croatian)

Huang, S., Tomljanovic, T., Tian, X., Wang, Y., Cao, X. (2016): The complete mitochondrial genome of natural Cobitis elongatoides (Cypriniformes: Cobitidae). Mitochondrial DNA, 27, 189-190.

Ištok, I. (2011): The Sutla River - one of the most biologically valuable watercourses. Hrvatska vodoprivreda, 197, 92-94. (in Croatian)

Ivakić, V. (1905): Pond fishing. Lovačko-ribarski viestnik, 14, 76-80. (in Croatian)

Ivančić, J. (1946): Amiurus nebulosus. Ribarstvo Jugoslavije, 1, 3, 30-31. (in Croatian)

Ivanović, B. (1972): Scardinius erythrophthalmus scardafa. Ichthyologia, 4, 1, 33-39. (in Croatian)

Ivelić, D., Jablan, I., Svjetličić, S., Piria, M. (2007): About loach in the Sava River. Ribarstvo, 65, 3, 99-110. (in Croatian with English abstract)

Jadan, M. (2008): Genetic structure of brown trout (Salmo trutta L.) populations in karstic rivers. PhD thesis, Faculty of Science, University of Zagreb. (in Croatian with English abstract)

Jadan, M., Čož-Rakovac, R., Topić Popović, N., StrunjakPerović, I. (2007): Presence of unexpected phylogenetic 
lineages of brown trout Salmo trutta L. in Gacka River, Croatia. Aquaculture Research, 38, 1682-1685.

Jadan, M, Strunjak-Perović, I, Topić Popović, N, ČožRakovac, R (2015): Three major phylogenetic lineages of brown trout (Salmo trutta Linnaeus, 1758) in the Krka River system (Croatia) revealed by complete mitochondrial DNA control region sequencing. Journal of Applied Ichthyology, 31, 192-196.

Jagodić, S. (1957): A letter from Osijek. Ribarski list, 1, 1213. (in Croatian)

Jakirčević, J. (2005): Fish fauna in the middle Sava River, near Davor. Odijel za biologiju, Sveučilište Josip Juraj Strossmayer u Osijeku, Osijek, 75pp. (in Croatian with English abstract)

Jakovlić, I., Gui, J. F. (2011): Recent invasion and low level of divergence between diploid and triploid forms of Carassius auratus complex in Croatia. Genetica, 139, 789-804.

Jakovlić, I., Piria, M., Šprem, N., Tomljanović, T., Matulić, D., Treer, T. (2015): Distribution, abundance and condition of invasive Ponto-Caspian gobies Ponticola kessleri Günther, 1861), Neogobius fluviatilis (Pallas, 1814), and Neogobius melanostomus (Pallas, 1814) in the Sava River basin, Croatia. Journal of Applied Ichthyology, 33, 888-894.

Jakovlić, I., Treer, T. (2001): Structure, growth and morphology fish populations from gravel-pit Vukovina. Ribarstvo, 59, 4, 142-149. (in Croatian with English abstract)

Jakovlić, I., Wu, Q. J., Treer, T., Šprem, N., Gui, J. F. (2013): Introgression evidence and phylogenetic relationship among three (Para)Misgurnus species as revealed by mitochondrial and nuclear DNA markers. Archives of Biological Sciences, 65, 1463-1467.

Jakšić, Ž., Hamer, B., Landeka, N., Batel, R. (2008): Western mosquitofish as a bioindicator of exposure to organochlorine compounds. Ecotoxicology and Environmental Safety, 71, 426-435.

Jakšić, G. (2016): Biological, ecological and genetic characteristics of invasive Ponto-Caspian gobies (Gobiidae) in the Sava River Basin in Croatia. PhD thesis. Faculty of Agriculture, University of Zagreb.

Jakšić, G., Jadan, M., Piria, M. (2016): The review of ecological and genetic research of Ponto-caspian gobies (Pisces, Gobiidae) in Europe. Croatian Journal of Fisheries, 74, 3, 110-123.

Jakšić, G., Maruškić Kulaš, M., Kuri, K., Petravić, J., Jarnjak, M., Željković, K., Piria, M. (2019): Age and growth of the Illyrian chub Squalius illyricus from the river Cetina. Proceedings of the $54^{\text {th }}$ Croatian \& $14^{\text {th }}$ International Symposium on Agriculture. Faculty of Agriculture, Croatia, 368-372.

Jalžić, B., Jalžić V, Miculinić, K. (2008): Rupećica cave and spring - Rupećica sinkhole and Green Lake. Speleolog, 56, 44-50. (in Croatian)

Janković, D., Karapetkova, M. (1992): Present status of the studies on range of distribution of Asian fish species Pseudorasbora parva (Schlegel) 1842 in Yugoslavia and Bulgaria. Ichthyologia, 24, 1, 1-9.

Jardas, I. (1996): The Adriatic ichthyofauna. Školska knjiga, Zagreb, 535pp. (in Croatian with English abstract)

Jardas, I., Pallaoro, A., Vrgoč, N., Jukić-Peladić, S., Dadić, V. (2008): Red Book of Marine Fish of Croatia. State Institute for Nature Protection, Zagreb, 396pp. (in Croatian with English abstract)

Jedlička, D. H. (1935): Important fishing waters of Vrbanska Banovina. Ribarski vjesnik, 13, 9-10, 225-230. (in Croatian)

Jedlička, D. H. (1954): The Neretva and its salmonids. Ribarski list, 3, 49-50. (in Croatian)

Jedlička, M. H. (1925): Softmouth trout Salar obtusirostris - Heck. Ribarski vjesnik, 3, 3, 44-46. (in Croatian)

Jedlička, M. H. (1926a): Marble trout. Športski ribar, 1, 1, 4-7. (in Croatian)

Jedlička, M. H. (1926b): Neretva. Športski ribar, 1, 4-5, 7-14. (in Croatian)

Jedlička, M. H. (1928): Softmouth trout. Ribarski list, 3, 1-2, 11-14. (in Croatian)

Jedlička, M. H. (1930): A letter from Zagreb. Ribarski list, 5, 5-6, 71-72. (in Croatian)

Jelačin, I. (1931): On the spawning of the Solin trout. Ribarski list, 6, 1-4, 23. (in Croatian)

Jelić, D., Krivek, G. (2017): Threatened fishes of the world: Telestes croaticus (Steindachner, 1866) (Cypriniformes: Cyprinidae). Croatian Journal of Fisheries, 75, 1, 25-29.

Jelić, D. (2012): New data on the distribution of Cottus gobio (Scorpaeniformes; Cottidae) in Croatia with special overview of the Adriatic basin. Ribarstvo, 70, 1, 1-8.

Jelić, D., Duplić, A., Ćaleta, M., Žutinić, P. (2008): Endemic fish species of the Adriatic wtareshed. Agencija za zaštitu okoliša, Zagreb, 77pp. (in Croatian with English abstract)

Jelić, D., Jelić, M. (2015): Telestes miloradi Bogutskaya, Zupancic, Bogut \& Naseka, 2012 and Delminichthys ghetaldii (Steindachner, 1882) re-discovered in Croatia, requiring urgent protection. Journal of Applied Ichthyology, 31, 1133-1136.

Jelić, D., Jelić, M., Žutinić, P. (2010): New data on ichthyofauna of River Česma (Central Croatia). Ribarstvo, 68, 68, 95-104. (in Croatian with English abstract)

Jelić, D., Jelić, M., Žutinić, P., Šimunović, I., Zupančič, P., Naseka, A. (2018): Distribution of endangered Italian gudgeon Romanogobio benacensis (Cypriniformes; Cyprinidae; Gobioninae) with remarks on distinguishing morphological characters. ZooKeys, 729, 103-127.

Jelić, D., Špelić, I., Žutinić, P. (2016): Introduced species community over-dominates endemic ichthyofauna of high Lika plateau (Central Croatia) over a 100 year period. Acta Zoologica Academiae Scientiarum Hungaricae, 62, 1-26. 
Jelić, D., Žutinić, P., Jelić, M. (2009): Characteristics and new data of river llova ichthyofauna (central Croatia). Ribarstvo, 67, 2, 53-61. (in Croatian with English abstract)

Jelić, M., Jelić, D., Žutinić, P., Ćaleta, M. (2012):. Fish fauna of the lower reaches of the river Drava and surrounding marshland habitats near Donji Miholjac (eastern Croatia). Ribarstvo, 70, 4, 153-167. (in Croatian with English abstract)

Jelkić, D., Opačak, A. (2013): A record of the North American paddlefish (Polyodon spathula Walbaum, 1872) in Croatia. Journal of Applied Ichthyology, 29, 1152-1153.

Jelkić, D., Opačak, A., Ozimec, S., Florijančić, T., Puškadija, Z., Bošković, I. (2010): Monitoring the Ichthyofauna in nature park Kopački rit (Croatia): in 2008. $38^{\text {th }}$ IAD Conference, Dresden, 1-5.

Jevtić, J. (1991): Choice of whitefish (Coregonus) for stocking of open and closed waters. Ribarstvo Jugoslavije, 46, 1-2, 14-26. (in Croatian with English abstract)

Jirásek, J., Fašaić, K. (2000): Sturgeons (Acipenseridae) relicts of prehistoric ichtiofauna). Ribarstvo, 58, 2, 4554. (in Croatian with English abstract)

Jugović, D. (2004): Ichthyofauna of the southern Psunj waters. Master's thesis, Faculty of Science, University of Zagreb. (in Croatian with English abstract)

Jurinac, A. E. (1882): A contribution to the understanding of the brook lamprey Petromyzon planeri Bl.. Rad Jugoslavenske akademije znanosti i umjetnosti, 61, 104-122. (in Croatian)

Jurinac, A. E. (1880): On the fish of the Drava, Plitvica and Bednja Rivers. Izvještaj Kraljevske Velike Gimnazije u Varaždinu 1879/80, 3-41. (in Croatian)

Jurinac, A. E. (1881): Continuation on the fish of the Drava, Plitvica and Bednja Rivers. Izvještaj Kraljevske Velike Gimnazije u Varaždinu 1880/81, 3-28. (in Croatian)

Jurinac, A. E. (1884): The vertebrates of the Varaždin area. Izvještaj Kraljevske Velike Gimnazije u Varaždinu 1883/84, 52-55. (in Croatian)

Jurinac, A. E. (1886): A contribution to the Croatian fauna of the Ogulin and Slunj areas and caves. Rad Jugoslavenske akademije znanosti i umjetnosti, 83, 86128. (in Croatian)

Jurinac, A. E. (1887): A contribution to the fauna of western Slavonia. Glasnik Hrvatskoga naravoslovnoga družtva, 1-3, 21-34. (in Croatian)

Juzbašić, V. (1928): The voracious blue bream. Priroda, 18, 2, 56. (in Croatian)

Kaitner. G. (1926a): Stocking the Maksimir Lakes with carp. Ribarski vjesnik, 4, 4, 32-34. (in Croatian)

Kaitner, G. (1926b): Spawning of rainbow trout. Ribarski vjesnik, 4, 4, 34-37. (in Croatian)

Kaitner, G. (1926c): The hunt for huchen. Ribarski vjesnik, 4, 4, 53-54. (in Croatian)

Kaitner, G (1928): The hunt for trout. Ribarski vjesnik, 6, 6,
6-7. (in Croatian)

Kaitner, G. (1929): Stocking with grayling. Ribarski vjesnik, 7, 7, 17-24. (in Croatian)

Kaitner, G. (1931): Removal of pike from the Gacka River. Ribarski vjesnik, 9, 9, 33-40. (in Croatian)

Kaitner, G. (1932): Pike season closed. Ribarski vjesnik, 10, 10, 12-13. (in Croatian)

Kaitner, J. (1929): Stocking crustaceans. Ribarski vjesnik, 7, 7, 25-26. (in Croatian)

Kajgana, Lj. (1996): Silver prussian carp and the damages it caused by its presence on fish farms. Ribarstvo, 54, 3, 131-134. (in Croatian with English abstract)

Kalous, L., Rylková, K., Bohlen, J., Šanda, R., Petrtýl, M. (2013): New mtDNA data reveal a wide distribution of the Japanese ginbuna (Carassius langsdorfii; Cyprinidae) in Europe. Journal of Fish Biology, 82, 2, 703-707.

Kapac, E. (1948): Fishing on the Orljava River. Ribarstvo Jugoslavije, 3, 7-8, 79. (in Croatian)

Kapetanović, D., Vardić l, Kurtović, B., Valić, D., Teskeredžić, E. (2008): Detection of the causative agent of furunculusis, Aeromonas salmonicida in salmonids of the Krka River. Veterinary Research Communications, 32, 131-135.

Kapetanović, N., Aganović, M., Vuković, T. (1966): On the specific shape of otoliths in individuals from three locla populations of Dalmatian barbelgudgeon (Aulopyge hügelii). Ribarstvo Jugoslavije, 21, 6, 134-137. (in Croatian)

Karaman, L. S. (1952): $12^{\text {th }}$ Contribution to the knowledge of the freshwater fish of Yugoslavia. Prirodoslovna istraživanja, 25, 117-128. (in Croatian)

Karaman, M. S. (1971): Süsswasserfische der Turkei 8. Revision der Barben Europas, Vorderasiens und Nordafrikas. Mitteilungen aus dell Hamburgischen Zoologischen Museum und Institut, 67, 175-254. (in German)

Karaman, M. S. (1972): Süsswasserfische der Turkei. 9 Teil. Revision einiger kleinwüchsiger Cyprinidengattungen Phoxinellus, Leucaspius, Acanthobrama usw. aus Südeuropa und Kleineasien. Mitteilungen aus den Hamburgischen Zoologischen Museum und Institut, 69, 115-155. (in German)

Karaman, S. (1922): Über eine neue Cobitis-Art aus Jugoslavien, Cobitis balcanica n. sp. Glasnik Hrvatskoga Prirodoslovnoga društva, 34, 307-310. (in German)

Karaman, S. (1923a): Die phylogenetische Bedeutung der Schuppen und Wirbelzahl bei Cyprinden. Glasnik zemaljskog Muzeja u Bosni i Hercegovini, 35, 183-190. (in German)

Karaman, S. (1923b): Über die Herkunft der Süßwasserfische unseres Karstes. Glasnik zemaljskog Muzeja u Bosni i Hercegovini, 35, 163-176. (in German)

Karaman, S. (1926): Les Salmonidés des Balkans. Glasnik skopskog naučnog društva, 2, 253-268. (in French)

Karaman, S. (1928): Contributions to the ichthyology 
of Yugoslavia. I. Glasnik Skopskog naučnog društva, Odelenje prirodnih nauka, 6, 147-176. (in Croatian)

Karaman, S. (1929): Über einige neue Fische aus Yugoslavien. Zoologischer Anzeiger 80, 171-173. (in German)

Karaman, S. (1932): New contributions to the knowledge of our salmonid fishes. Ribarski list, 7, 9-10, 97-99. (in Croatian)

Karaman, S. (1936): 10th contribution to the knowledge of the freshwater fishes of Yugoslavia. Glasnik skopskog naučnog društva, 17, 55-64. (in Croatian)

Karaman, S. (1938): 11. Beitrag zur Kenntnis der Susswasserfische Jugoslawiens. Glasnik skopskog naučnog društva, 18, 131-139. (in German)

Karaman, S.M (1966): Beitrag zur Kenntnis der Salmoniden Südeuropas. Hydrobiologia, 28, 1-41. (in German)

Katurić, M. (1883): Notizie Zoologiche. Bollettino della Società adriatica di scienze naturali in Trieste, 8, 123131. (in Italian)

Katurić, M. (1887): Cenni ittiologico-erpetologici. Glasnik Hrvatskoga naravoslovnoga društva, 2, 111-118. (in Italian) (in Italian)

Katurić, M. (1896a): Zoological news. Contributions in the study of nature. Državna štamparija Kraljevine Srbije, Beograd, 11-17. (in Croatian)

Katurić, M. (1896b): Zoological news. Contributions in the study of nature. Državna štamparija Kraljevine Srbije, Beograd, 18-21. (in Croatian)

Katurić, M. (1896c): Ichthyology and herpetology research. Contributions in the study of nature. Državna štamparija Kraljevine Srbije, Beograd, 27-33. (in Croatian)

Katurić, M. (1896d): Ichthyology and herpetology research. Contributions in the study of nature. Državna štamparija Kraljevine Srbije, Beograd, 36-37. (in Croatian)

Katurić, M. (1896e): Anguilla fluviatilis of Dalmatia. Contributions in the study of nature. Državna štamparija Kraljevine Srbije, Beograd, 38-51. (in Croatian)

Katurić, M. (1896f): Discussion on the marine trout Trutta adriatica Kolomb. Contributions in the study of nature. Državna štamparija Kraljevine Srbije, Beograd, 83-94. (in Croatian)

Katurić, M. (1903): New finds of the Atlantic sturgeon, Acipenser sturio L.. Rad Jugoslavenske akademije znanosti i umjetnosti, 33, 1-6. (in Croatian)

Katurić, M. (1907): Report by Prof. Katurić during the first assembly of the Society of theCity Natural History Museum in Zadar, 30 December 1906, with a list of the natural artefacts collected to date. Tiskarnica P. Jankovića, Zadar, 35pp. (in Croatian)

Katurić, M. (1908): Zoological contributions. Rad Jugoslavenske akademije znanosti i umjetnosti, 43, 3237. (in Croatian)

Kažić, D., Marić, D., Ulićević, D. (1990): Cage farming of the brook trout (Salvelinus fontinalis Mitchill 1815) in
Skadar Lake. Ribarstvo Jugoslavije, 45, 5-6, 101-105. (in Croatian)

Kempf, J. (1910): Požega - geographic notes from the surrounding areas and the history of the free and royal town of Požega and of Požega County. Štamparija Hrvatske tiskare i knjižare, Požega, 777pp. (in Croatian)

Kerestessy, K., Beliczky, G., Kiss, G. (2009): Adatok a Mura Letenyei szakaszanák halfaunájához. Pisces Hungarici, 3, 21-25. (in Hungarian)

Kerovec, M., Mrakovčić, M., Schneider, D., Tomašković, N. (1998): Occurrence of Chondrostoma phoxinus in Croatia. Folia Zoologica, 47, 39-43.

Kesterčanek, F. X. (1886): Our fisheries. Šumarski list, 10, 25-33. (in Croatian)

Ketmaier, V., Bianco, P. G., Cobolli, M., Krivokapic, M., Caniglia, R., De Matthaeis, E. (2004): Molecular phylogeny of two lineages of Leuciscinae cyprinids (Telestes and Scardinius): from the peri-Mediterranean area based on cytochrome b data. Molecular Phylogenetics and Evolution, 32, 1061-1071.

Ketmaier, V., Bianco, P. G., Durand, J-D. (2008): Molecular systematics, phylogeny and biogeography of roaches (Rutilus, Teleostei, Cyprinidae). Molecular Phylogenetics and Evolution, 49, 362-367.

Kišpatić, M. (1893): Fish, natural history and cultural notes. Matica Hrvatska, Zagreb, 455pp. (in Croatian)

Klašterka, V. (1979): Sports fishing on rivers and lakes. Svetin, L. (ed), Nakladni zavod Znanje, Zagreb, 310pp. (in Croatian)

Knežević, K. (2007): The nutrition of a Brown trout (Salmo trutta L. 1758) and Rainbow trout (Oncorhynchus mykiss Walbaum 1792) in the river Gacka. Master's thesis, Faculty of Science, University of Zagreb. (in Croatian with English abstract)

Knežević, P. (1952): New trout spawning grounds in Vrbovsko. Ribarstvo Jugoslavije, 7, 1-2, 11-12. (in Croatian)

Knežević, P. (1963a): Experiences and issues ni the acclimatisation of rainbow trout. Ribarstvo Jugoslavije, 18, 1, 6-8. (in Croatian)

Knežević, P. (1963b): Experiences and issues ni the acclimatisation of rainbow trout. Ribarski list, 1, 11-14. (in Croatian)

Knop, F. (1947a): Asp (Aspius rapax). Ribarstvo Jugoslavije, 2, 5, 78. (in Croatian)

Knop, F. (1947b): Chub (Squalius cephalus). Ribarstvo Jugoslavije, 2, 5, 78-79. (in Croatian)

Knop, F. (1947c): On the huchen (Salmo hucho). Ribarstvo Jugoslavije, 2, 5, 78. (in Croatian)

Kolak, A., Treer, T., Aničić, I., Safner, R. (1999): Monitoring the genotoxicity of the river Sava by micronuklei in chub (Leuciscus cephalus). Cytobios, 99, 135-142.

Kolaković, S. (1972): „Škobaljaš“ sufficient qualifications for successful marine fishing. Ribarski list, 1, 106. (in Croatian)

Kolar-Dimitrijević, M. (1994): Serbian view of agricultural 
economy in several Sava districts in 1925/1926. (Zagreb, Srijem i Tuzla region). Povijesni prilozi, 13, 13, 179-218. (in Croatian with English abstract)

Kolarević, S., Aborgiba, M., Kračun-Kolarević, M., Kostić, J., Simonović, P., Simić, V., Milošković, A., Reischer, G., Farnleitner, A., Gačić, A., Milačič, R., Zuliani, T., Vidmar, J., Pergal, M., Piria, M., Paunović, M., Vuković-Gačić, B. (2016): Evaluation of genotoxic pressure along the Sava River. PLOS ONE, 15, 1-23.

Koller, O. (1926): Eine kritische Übersicht über die bisher beschriebenen mittel- und südeuropäischen Arten der Cyprinidengattung Barbus Cuv. Sitzungsberichte der Mathematisch-Naturwissenschaftlichen Klasse, 1, 167202. (in German)

Koller, O. (1928): Die geographische Verbreitung der Süßwasserfische in Südeuropa. Sitzungsberichte der Akademie der Wissenschaften mathematischnaturwissenschaftliche Klasse, 137, 627-642. (in German)

Kolombatović, G. (1881): Pesci delle acque di Spalato e catalogo degli anfibi e dei rettili dei contorni di Spalato. Godišnje Izvješće C. i K. Velike Realke u Splitu školska godina 1880-1881, 1-29. (in Italian)

Kolombatović, G. (1882): Fische, welche in den Gewässern von Spalato beobachtet und überhaupt im adriatischen Meere registrirt wurden. Zannoni, Split, 61pp. (in German)

Kolombatović, G. (1884): Aggiunte ai vertebrati della Dalmazia. Godišnje Izvješće C. i K. Velike Realke u Splitu školska godina 1883-1884, 3-28. (in Italian)

Kolombatović, J. (1886a): Directory of Dalmatian vertebrates. $2^{\text {nd }}$ part: Amphibians, Reptiles and Fish. Godišnje Izvješće C. i K. Velike Realke u Splitu školska godina 1885-1886, 3-20. (in Croatian)

Kolombatović, J. (1886b): Terze aggiunte ai vertebrati della Dalmazia. Godišnje Izvješće C. i K. Velike Realke u Splitu školska godina 1885-1886, 21-32. (in Italian)

Kolombatović, G. (1888): Catalogus vertebratorum dalmaticorum. Godišnje Izvješće C. i K. Velike Realke u Splitu školska godina 1887-1888, 3-31. (in Latin)

Kolombatović, G. (1890): Notizie ittiologiche. Seconda trotta marina pescata nel mare di Vranjic Trutta adriatica n. sp. Glasnik Hrvatskoga naravoslovnoga družtva, 1-4, 165-167. (in Italian)

Kolombatović, G. (1891): Gobies (Gobii) of the marine area surrounding Split in Dalmatia. Godišnje Izvješće C. i K. Velike Realke u Splitu školska godina 1890-1891, 1-29. (in Croatian)

Kolombatović, G. (1892): Blennies (Blenniini) of the marine area surrounding Split. Godišnje Izvješće C. i K. Velike Realke u Splitu školska godina 1891-1892, 3-14. (in Croatian)

Kolombatović, G. (1893): New discoveries of the Dalmatian vertebrates. Godišnje Izvješće C. i K. Velike Realke u Splitu školska godina 1892-1893, 3-27. (in Croatian)

Kolombatović, G. (1894): On the records of species of cephalopods and vertebrates of the Adriatic Sea. Godišnje Izvješće C. i K. Velike Realke u Splitu školska godina 1894-1895, 3-54. (in Croatian)

Kolombatović, G. (1895): On certain vertebrates. Godišnje Izvješće C. i K. Velike Realke u Splitu školska godina 1894-1895, 3-32. (in Croatian)

Kolombatović, G. (1896): Zoological news from Dalmatia. Godišnje izvješće C. i K. velike realke u Splitu školska godina 1895. i 1896. 3-24. (in Croatian)

Kolombatović, G. (1902): Contribuzioni alla fauna dei Vertebrati della Dalmazia. Glasnik Hrvatskoga naravoslovnoga društva, 4-6, 22-37. (in Italian)

Kolombatović, G. (1903): Contribuzioni alla fauna dei vertebrati della Dalmazia. Pesci. Glasnik Hrvatskoga naravoslovnoga društva, 1, 182-200. (in Italian)

Kolombatović, G. (1907): Contribuzioni alla fauna dei vertebrati della Dalmazia. Glasnik Hrvatskoga naravoslovnoga društva, 19, 1-24. (in Italian)

Kopjar, N., Mustafić, P., Zanella, D., Buj, I., Ćaleta, M., Marčić, Z., Milić, M., Dolenec, Z., Mrakovčić, M. (2008): Assessment of DNA integrity in erythrocytes of Cobitis elongata affected by water pollution: the alkaline comet assay study. Folia Zoologica, 57, 120-130.

Koritnik, M. (1934): Memories from Čabranka. LovačkoRibarski vjesnik, 43, 436-440. (in Croatian)

Koritnik, M. (1973): Grayling. Ribarski list, 3, 80. (in Croatian)

Kosić, B. (1889): Materials for the Dubrovnik nomenclature and fish fauna. Glasnik Hrvatskoga naravoslovnoga društva 1, 273-299. (in Croatian)

Kosić, B. (1891): Addition to the Dubrovnik nomenclature and fish fauna. Glasnik Hrvatskoga naravoslovnoga društva, 1-6, 205-215. (in Croatian)

Kosić, B. (1898): New material for the Dubrovnik nomenclature and fish fauna. Glasnik Hrvatskoga naravoslovnoga društva, 1-5, 77-88. (in Croatian)

Kosić, B. (1903): The fishes of Dubrovnik. Rad Jugoslavenske akademije znanosti i umjetnosti, 155, 1-48. (in Croatian)

Kosorić, Đ. (1969): Some data on the distribution and migration of the Neretva marble trout (Salmo marmoratus Cuv.): in the Neretva basin. Glasnik Zemaljskog muzeja Bosne i Hercegovine u Sarajevu 8, 79-82. (in Croatian)

Kosorić, Đ. (1978): Composition of the fish population of Hutovo Blato. Godišnjak BIUS-a, 26, 69-81. (in Croatian)

Kosorić, V. (1955): Una. Ribarski list, 2, 25-34. (in Croatian) Kosorić, V. (1958): Una River. Ribarski list, 2-3, 47-55. (in Croatian)

Kotlik, P., Tsigenopoulos, C., Rab, P., Berrebi, P. (2002): Two new Barbus species from the Danube River basin, with redescription of Barbus petenyi (Teleostei: Cyprinidae). Folia Zoologica, 51, 227-240.

Kottelat, M. (1984): Catalogue des types du Musée d'histoire naturelle de Neuchâtel. I, Pisces, Bulletin de la Société Neuchâteloise des Sciences Naturelles, 107, 
143-153 (in French)

Kottelat, M. (1997): European Freshwater fishes. An heuristic checklist of the freshwater fishes of Europe (exclusive of former USSR), with an introduction for non-systematists and comments on nomenclature and conservation. Biologia (Bratislava), Section Zoology, 52, $1-271$.

Kottelat, M. (2012): Conspectus cobitidum*: an inventory of the loaches of the world (Teleostei: Cypriniformes: Cobitoidei). The Raffles Bulletin of Zoology Supplement, 26, 1-199.

Kottelat, M., Freyhof, J. (2007): Handbook of European Freshwater Fishes. Kottelat Cornol, Switzerland. Freyhof Berlin, Germany, 646pp.

Kovačević, J. (1952): Neretva wetlands. Priroda, 39, 1, 1316. (in Croatian)

Kovačić, M. (1998): Ichthyological collection (Cyclostomata, Selachii, Osteichthyes) of the natural history museum Rijeka. In: Arko-Pijevac, M. (ed), Prirodoslovna istraživanja riječkog područja. Prirodoslovni muzej Rijeka, Rijeka, 685-698.

Kovačić, M. (2005a): A new species of Knipowitschia (Gobiidae) from Dalmatia, Croatia. Cybium, 29, 3, 275280.

Kovačić, M. (2005b): Morphological variability of Pomatoschistus canestrini (Gobiidae) with the reduction of squamation and head canals. Cybium, 29, 4, 373-379.

Kovačić, M. (2008): The key for identification of Gobiidae in the Adriatic Sea. Acta Adriatica, 49, 3, 245-254.

Kovačić, M., Pallaoro, A. (2003): Is Knipowitschia caucasica-like form from the Adriatic sea a new goby species? Evidence from a morphological approach in the eastern Adriatic sea. Cybium, 27, 2, 131-136.

Krajačić (1932): A letter from St. Ivan Zelina. Ribarski list, 7, 9-10, 115. (in Croatian)

Kraljević, M., Dulčić, J. (1996): Age, growth and mortality of the of the golden grey mullet Liza aurata (Risso, 1810) in the eastern Adriatic. Archive of fishery and marine research, 44, 69-80.

Kraljić, M. (2010): Morphological traits, growth and population structure of Danube barbel (Barbus balcanicus, Actinopterygii) in Croatia. Master's thesis, Faculty of Science, University of Zagreb. (in Croatian)

Krasnići, N., Dragun, Z., Erk, M., Raspor, B. (2013): Distribution of selected essential (Co, Cu, Fe, Mn, Mo, $\mathrm{Se}$, and $\mathrm{Zn}$ ): and nonessential ( $\mathrm{Cd}, \mathrm{Pb})$ : trace elements among protein fractions from hepatic cytosol of European chub (Squalius cephalus L.). Environmental Science and Pollution Research, 20, 4, 2340-2351.

Krauss, H. (1932): Huchen in the Drava. In: Thaller, Z. (ed), Vode i ribe Jugoslavije u slici i riječi. Tipografija, Zagreb, 162-174. (in Croatian)

Krpo-Ćetković, J., Stamenković, S. (1996): Morphological differentiation of the pikeperch Stizostedion lucioperca (L.) populations from the Yugoslav part of Danube.
Annales Zoologici Fennici, 33, 711-723.

Kuhar, D., Huber, Đ. (1989): Parasitic helminths of trout in the Plitvice Lakes National Park. Periodicum Biologorum, 91, 1, 115-116.

Kulčar, G. (1911): Varaždin. Lovačko-Ribarski vjesnik, 20, 117. (in Croatian)

Kurtović, B., Kapetanović, D., Matašin, Ž. (2007): Comparison of the histological changes in organs of chub (Squalius cephalus) with the number of bacteria in the water of the Sava River. Ribarstvo, 65, 4, 127140. (in Croatian with English abstract)

Kurtović, B., Vardić, I., Valić, D., Kapetanović, D., Teskeredžić, Z., Teskeredžić, E. (2009): Health status of chub (Squalius cephalus) in relation to the water quality of Sava River. Ribarstvo, 67, 2, 63-75. (in Croatian with English abstract)

Kužir, S., Gjurčević, E., Nejedli, S., Baždarić, B., Kozarić, Z. (2012): Morphological and histochemical study of intestine in wild and reared European eel (Anguilla anguilla L.). Fish Physiology and Biochemistry, 38, 625633.

Labak, I. (2011): Diversity in expression of polysialic proteins in teleostean brain. PhD thesis, Medical faculty, University Josip Juraj Strossmayer.

Landeka, N., Podnar, M., Jelić, D. (2015): New data on the taxonomic status and distribution of Gambusia sp. in Croatia and Bosnia and Herzegovina. Periodicum Biologorum, 117, 3, 415-424.

Lang, M. (1911): Samobor. Folk life and customs. Zbornik za narodni život i običaje južnih Slavena, 16, 1-128. (in Croatian)

Langhoffer, A. (1901): From the literature. Glasnik Hrvatskoga naravoslovnoga društva, 1-3, 90. (in Croatian)

Langhoffer, A. (1904): List of fish received at the National Zoological Museum in Zagreb by the end of the year 1900. Glasnik Hrvatskoga naravoslovnoga društva, 16, 148-169. (in Croatian)

Langhoffer, A. (1910): Eel. Lovačko-Ribarski vjesnik, 19, 111-114. (in Croatian)

Langhoffer, A. (1915): Notizen aus dem kroatische zoologischen Landesmuseum. Glasnik Hrvatskoga naravoslovnoga društva, 27, 58-59. (in German)

Lanza, F. (1842): Saggio storico statistico-medico sopra l'antica città di Narona e lo stato presente del suo territorio corredato di alcune inedite antiche iscrizioni e di una carta topografica litografata. Marsigli, Bologna, 143pp. (in Italian)

Largaiolli, V. (1904): Notizie fisiche e biologiche sul lago Cepich in Istria. Coana, Parenzo, 3-32. (in Italian)

Leiner, S (1984): Preliminary research of the ichthyofauna of the terrestrial waters of Istria. Bilten Društva ekologa Bosne i Hercegovine, 3, 37-40. (in Croatian)

Leiner, S. (1985): Ichthyofauna of the terrestrial waters of Istria. Master's thesis, Faculty of Science, University of Zagreb. (in Croatian with English abstract) 
Leiner, S. (1995): The status of European mudminnow, Umbra krameri Walbaum, 1792, in Croatia. Annalen des Naturhistorischen Museums in Wien, 97, B, 486490.

Leiner, S. (1998a): Endangered ichthyocoenoses of the Sava and Odra rivers in the Turopolje area. Natura Croatica, 7, 1, 31-43.

Leiner, S. (1998b): Fishes of the Croatian part of the Neretva River basin. Časopis za književnost i znanost, 9 , 4, 245-252. (in Croatian)

Leiner, S. (1999): Management and ichthyological research in the waters of „Plitvice Lakes" National Park. Priroda, 89, 861, 37-39. (in Croatian)

Leiner, S. (2003): Ein großer natürlicher Fischlaichplatz. Euro city, putna revija HŽ-putničkog prijevoza, 26-28. (in German)

Leiner, S., Popović, J. (1984): The genus Leuciscus (Cyprinidae, Pisces) in the waters of the Adriatic Basin, with reference to the finds of $L$. svallize (Heckel \& Kner, 1858) and L. souffia Risso, 1826 in the Cetina. Ichthyologia, 16, 1-2, 111-120. (in Croatian)

Leiner, S., Povž, M. (1993): Freshwater fish (Pisces) of the Istrian Peninsula. Ichthyos, 12, 23-33. (in Croatian)

Leiner, S., Povž, M. (1994): The freshwater fishes of Istrian Peninsula. Periodicum Biologorum, 96, 4, 431-434.

Leiner, S., Povž, M., Mrakovčić, M. (1995): Freshwater fish in Istrian Peninsula. Annales for Istrian and Mediterranean Studies, Series Historia Naturalis, 7, 215-222.

Lelek, A. (1987): Freshwater fishes of Europe: Band 9, Threatened fishes of Europe. AULA-Verlag, Wiebelsheim, 343pp.

Lenhardt, M., Jarić, I., Bojović, D., Cvijanović, G., Gačić, Z. (2006): Past and current status of sturgeon in the Serbian part of the Danube River. Proceedings of the $36^{\text {th }}$ International Conference of IAD. Austrian comittee Danube research/IAD, Vienna, 148-151.

Lipej, L., Dulčić, J. (2010): Checklist of the Adriatic Sea Fishes. Zootaxa, 2589, 1-92.

Lisjak, D., Zanella, D., Mustafić, P., Ćaleta, M., Buj, I., Mihinjač, T., Mrakovčić, M., Marčić, Z. (2015): First record of three-spined stickleback (Gasterosteus aculeatus Linnaeus, 1758) in the Danube basin of Croatia. Croatian Journal of Fisheries, 73, 70-72.

Livojević, Z. (1949): Opening of the freshwater fish farm in the Raša River. Ribarstvo Jugoslavije, 4, 5, 48. (in Croatian)

Livojević, Z. (1962a): Vrana Lake and its environs. Morsko Ribarstvo, 14, 11-12, 13-15. (in Croatian)

Livojević, Z. (1962b): Vrana Lake and its environs. Ribarstvo Jugoslavije, 17, 4, 101-103. (in Croatian)

Lorini, P. (1910): Eels in Vrana Lake on the island of Cres. Lovačko-Ribarski vjesnik, 20, 72. (in Croatian)

Lorini, P. (1921): Fishing in the Neretva River. LovačkoRibarski vjesnik, 29, 19-25. (in Croatian)

Lovrić, S. (1938): On the breeding and protection of freshwater fish in the Ličanka and Lokvarka Rivers. Lovačko-Ribarski vjesnik, 47, 493-498. (in Croatian)

Lucu, Č., Marsoni, S. S. (2013): Tribute to Professor Massimo Sella - Former Scientist and Director of the Marine Biological Station in Rovinj - On the occassion of 120th Anniversary of marine research in Rovinj (1891-2011). Periodicum Biologorum, 115, 1, 105-108.

Lujić, J., Kohlmann, K., Kersten, P., Marinović, Z., Ćirković, M., Simić, V. (2017): Phylogeographic identification of tench Tinca tinca (L, 1758) (Actinopterygii: Cyprinidae) from the northern Balkans and adjacent regions and its implications for conservation. Zoological Studies, 56, 1-9.

MacCrimmon, H. R. (1971): World distribution of rainbow trout (Salmo gairdneri). Journal of the Fisheries Research Board of Canada, 5, 663-704.

Maitland, P., Crivelli, A. (1996): Conservation of freshwater fish. Conservation of Mediterranean wetlands. No. 7, Tour du Valat Fondation, Arles, 94pp.

Majer, J. (1998): Adatok a Dráva és a Dráva menti területek hal-, kétéltű- és hüllőfaunájához (Pisces, Amphibia, Reptilia). Dunántúli Dolgozatok Természettudományi soroza, 9, 431-440. (in Hungarian)

Majer, J. (2001): Checklist of lamprey of Somogy county (Cyclostomata, Hyperontia: Petromyzoidae). Natura Somogyiensis, 1, 437-438.

Majnarić, K. (2009): Integrity of chub (Leuciscus cephalus L.) erythrocytes DNA as an indicator of effects of genotoxic pollution of river Kupa. Master's thesis, Faculty of Science, University of Zagreb. (in Croatian with English abstract)

Mäkinen, H. S., Merilä, J. (2008): Mitochondrial DNA phylogeography of the three-spined stickleback (Gasterosteus aculeatus) in Europe-Evidence for multiple glacial refugia. Molecular Phylogenetics and Evolution, 46, 167-182.

Maldini, R. (1933): White catfish. Ribarski list, 8, 11-12, 139. (in Croatian)

Maldini, R. (1935a): Folk names of fish (Freshwater fish). Ribarski list, 10, 10, 79-80. (in Croatian)

Maldini, R. (1935b): Folk names of fish (Freshwater fish). Ribarski list, 10, 11, 91-92. (in Croatian)

Maldini, R. (1935c): Folk names of fish (Freshwater fish). Ribarski list, 10, 12, 101-102. (in Croatian)

Maldini, R. (1936a): Folk names of fish (Freshwater fish). Ribarski list, 11, 1-2, 7-8. (in Croatian)

Maldini, R. (1936b): Folk names of fish (Freshwater fish). Ribarski list, 11, 3, 18-20. (in Croatian)

Maldini, R. (1936c): Folk names of fish (Marine fish). Ribarski list, 11, 4-5, 32-33. (in Croatian)

Maldini, R. B. (1937): Fishing on the Drava near Osijek. Ribarski list, 12, 8-9, 42-44. (in Croatian)

Maldini, R. B. (1938a): European river lamprey (Petromyzon fluviatilis). Ribarski list, 13, 1-2, 4-5. (in Croatian)

Maldini, R. B. (1938b): River and marine animals based on the folk names with notes of places and regions. 
Ribarski list, 13, 5-6, 30-31. (in Croatian)

Maldini, R. B. (1938c): River and marine animals based on the folk names with notes of places and regions. Ribarski list, 13, 7-8, 45-46. (in Croatian)

Malnar, L. (1989): Bacterial diseases of the salmonids of the Krka River and its mouth. Master's thesis, Faculty of Science, University of Zagreb. (in Croatian)

Marčić Brusina, Lj. (1963): From the former Drijeva port to the sea. Priroda, 50, 9-10, 278-280. (in Croatian)

Marčić, Z. (2013): Taxonomic, biological and ecological characteristics of the genus Telestes Bonaparte, 1837 (Actinopterygii) in the area of Velika Kapela and Mala Kapela mountains. PhD thesis, Faculty of Science, University of Zagreb. (in Croatian with English abstract) Marčić, Z., Buj, I., Duplić, A., Ćaleta, M., Mustafić, P., Zanella, D., Zupančič, P., Mrakovčić, M. (2011): A new endemic cyprinid species from the Danube drainage. Journal of Fish Biology, 79, 418-430.

Marčić, Z., Ćaleta, M., Buj, I., Zanella, D., Mustafić, P., Mihinjač, T., Mrakovčić, M. (2015): New records of Aphanius fasciatus (Valenciennes, 1821) along the eastern coast of the Adriatic sea in Croatia. Croatian Journal of Fisheries, 73, 124-127.

Marčić, Z., Milković, A., Ćaleta, M., Buj, I., Zanella, D., Mustafić, P. (2017): Reproductive biology of the endemic dace Telestes karsticus Marčić and Mrakovčić, 2011 (Cyprinidae, Leuciscinae) in Croatia. Journal of Applied Ichthyology, 33, 203-208.

Marčić, Z., Mustafić, P., Zanella, D., Ćaleta, M., Buj, I., Mrakovčić, M. (2012): Changes and structure of ichthyofauna during the 50 years of existence of the hydro accumulation Bajer and Lokva. In: Arko-Pijevac, M., Surina, B. (eds), Prirodoslovna istraživanja riječkog područja II. Prirodoslovni muzej Rijeka, Rijeka, 199204. (in Croatian with English abstract)

Marčić, Z., Sučić, I., Ćaleta, M., Buj, I., Mustafić, P., Zanella, D. (2017): Seasonal profile of the diet of the dace Telestes karsticus Marčić \& Mrakovčić, 2011 (Cyprinidae, Leuciscinae) endemic to Sušik Creek, Croatia. Journal of Applied Ichthyology, 33, 943-949.

Marešová, E., Delić, A., Kostov, V., Marić, S., Mendel, J., Šanda, R. (2011): Genetic diversity of Sabanejewia balcanica (Actinopterygii: Cobitidae) in the western Balkans and comparison with other regions. Folia Zoologica, 60, 335-342.

Marević, I. (2010): Marketing of natural resources and the traditional cuisine of the Neretva Delta. In: Glamuzina, B., Dulčić, J. (eds), Ribe i Ribarstvo, rijeke Neretve. Sveučilište u Dubrovniku i Dubrovačko neretvanska županija, Dubrovnik, 160-171pp. (in Croatian)

Marguš, D. (2008): Skradin waterfall. Hrvatska vodoprivreda, 183, 33-39. (in Croatian)

Marić, D. (2010): Rutilus albus sp. n (Teleostei: Cyprinidae) from Lake Skadar. Periodicum Biologorum, 112, 2, 153158.

Marić, S., Stanković, D., Wanzenböck, J., Šanda, R., Erős,
T., Péter, T., Specziár, A., Sekulić, N., Bănăduc, D., Ćaleta, M., Trombitsky, I., Galambos, L., Sipos, S., Snoj, A. (2017): Phylogeography and population genetics of the European mudminnow (Umbra krameri) with a time-calibrated phylogeny for the family Umbridae. Hydrobiologia, 792, 151-168.

Marijić Filipović, V. (2009): Distribution of selected essential and toxic metals in tissue, cytosol and intestinal parasites in the digestive tract of chub (Squalius cephalus L.). PhD thesis, Faculty of Science, University of Zagreb. (in Croatian with English abstract)

Marjanović, J. (1954): Catch of predatory fish in the Sisak region. Ribarstvo Jugoslavije, 9, 1, 23. (in Croatian)

Marko, S., Debeljak, Lj., Bralić, V., Sabioncello, I. (1968): Limnological characteristics of the Peruća reservoir. Ribarstvo Jugoslavije, 23, 2, 38-44. (in Croatian)

Marko, S., Stoisavljević, J. (1972): Results of spawning of the grass carp in the Grudnjak fish farm. Ribarstvo Jugoslavije, 27, 6, 139-140. (in Croatian)

Marková, S., Šanda, R., Crivelli, A., Shumka, S., Wilson, I. F., Vukić, J., Berrebi, P., Kotlík, P. (2010): Nuclear and mitochondrial DNA sequence data reveal the evolutionary history of Barbus (Cyprinidae) in the ancient lake systems of the Balkans. Molecular Phylogenetics and Evolution, 55, 488-500.

Marr, S. M., Olden, J. D., Leprieur, F., Arismendi, I., Ćaleta, M., Morgan, D. L., Nocita, A., Šanda, R., Tarkan, A. S., García-Berthou, E. (2013): A global assessment of freshwater fish introductions in mediterranean-climate regions. Hydrobiologia, 719, 317-329.

Martinović, D. (1995): Taxonomic specificities of the species Scardinius erythrophthalmus L. in the Adriatic basin. Master's thesis, Faculty of Science, University of Zagreb. (in Croatian with English abstract)

Martinović, D., Čaušić, N., Mikuška, T. (1992): Lonjsko Polje - not just a haven for birds (2). Priroda, 82, 9-10, 22-25. (in Croatian)

Mateš, A. (2004): The Enchanted Angler: records, experiences and catches of a fishing enthusiast. J\&B, Zagreb, 192pp. (in Croatian)

Mateš, A. (2008): The Enchanted Angler - Hunchens and graylings. J\&B, Zagreb, 216pp. (in Croatian)

Matić-Skoko, S., Pehardam, M., Pallaoro, A., Franičević, M. (2005): Species composition, seasonal fluctuations, and residency of inshore fish assemblages in the Pantan estuary of the eastern middle Adriatic. Acta Adriatica, 2, 201-212.

Matulić, D., Šprem, N., Piria, M., Tomljanović, T., Treer, T., Safner, R., Aničić, I. (2011): Analysis of recreational fisheries in the Croatian areas of the Sava and Danube Rivers. Agriculturae Conspectus Scientificus, 75, 183190.

Medić, M. (1896): Ichthyological notes. Rad Jugoslavenske akademije znanosti i umjetnosti, 126, 83-109. (in Croatian)

Medić, M. (1898): Roach. Rad Jugoslavenske akademije 
znanosti i umjetnosti, 135, 199-208. (in Croatian)

Medić, M. (1900): Nase. Nastavni vjesnik, 8, 463-469. (in Croatian)

Medić, M. (1901a): Trout. Nastavni vjesnik, 9, 157-159. (in Croatian)

Medić, M. (1901b): Second round of ichthyological notes. Rad Jugoslavenske akademije znanosti i umjetnosti, 30, 139-191. (in Croatian)

Medić, M. (1902): Ziege (Pelecus cultratus Linné) and Cactus roach (Leuciscus virgo Heckel). Glasnik Zemaljskog muzeja u Bosni i Hercegovini, 1, 113-122. (in Croatian)

Medić, M. (1905): Fish from the Kupa River in the Brod valley. Nastavni vjesnik, 13, 490-491. (in Croatian)

Meštrov, M., Justić, D. (1983): Changes in the ecological properties in the Jezero reservoir on the island of Krk. Ekologija, 1, 65-74. (in Croatian)

Mičetić, V., Bučar, M., Ivković, M., Piria, M., Krulik, I., Mihoci, I., Delić, A., Kučinić, M. (2008): Feeding ecology of Sabanejewia balcanica and Cobitis elongata in Croatia. Folia Zoologica, 57, 181-190.

Mihailović, M., Blagojević, D., Ogrinc, N., Simonović, P., Simić, V., Vidaković, V., Dinić, S., Uskoković, A., Grdović, N., Arambašić-Jovanović, J., Đorđević, M., Tolić, A., Kračun-Kolarević, M., Kolarević, S., Piria, M., Paunović, M. (2016): Biochemical indicators and biomarkers in chub (Squalius cephalus L.) from the Sava River. Science of the Total Environment, 540, 368-376.

Mihaljević, M., Opačak, A., Bogut, I., Novoselić, D. (1996): Quality of water and the ichthiofauna of the Southern Lake in Osijek - the conditions for biomanipulation. Ribarstvo, 54, 2, 55-64. (in Croatian with English abstract)

Mihinjač, T., Marčić, Z., Mrakovčić, M., Mustafić, P., Zanella, D., Buj, I., Ćaleta, M. (2015a): Threatened fishes of the world: Cobitis jadovaensis Mustafić \& Mrakovčić, 2008 (Cyprinidae). Croatian Journal of Fisheries, 73, 1, 46-47.

Mihinjač, T., Marčić, Z., Mrakovčić, M., Mustafić, P., Zanella, D., Ćaleta, M. (2014): Threatened fishes of the world: Telestes turskyi (Heckel, 1843) (Cyprinidae). Croatian Journal of Fisheries, 72, 3, 123-124.

Mihinjač, T., Marčić, Z., Buj, I., Zanella, D., Mustafić, P., Mrakovčić, M., Ćaleta, M. (2015b): Threatened fishes of the world: Delminichthys krbavensis (Zupančič \& Bogutskaya, 2002) (Cyprinidae). Croatian Journal of Fisheries, 73, 1, 33-34.

Mijošek, T., Filipović Marijić, V., Dragun, Z., Krasnići, N., Ivanković, D., Erk, M. (2019): Evaluation of multibiomarker response in fish intestine as an initial indication of anthropogenic impact in the aquatic karst environment. Science of the Total Environment, 660, 1079-1090.

Mikavica, D., Muhamedagić, S., Dizdarević, F., Savić, N. (2001): Idioecological characteristics of adriatic trout Salmothymus obtusirostris oxyrhynchus (Steindachner, 1882). Symposium of livestock production with international participation, Struga, 23-25.

Mikuska, J. (1979): Ecological properties and protection of the Kopački Rit Special Zoology Reserve, with special emphasis on the ecology of the vertebrates. PhD thesis, Faculty of Science, University of Zagreb. (in Croatian)

Mikuska, J. (1983a): Ecological properties of the fish fauna in the Kopački Rit Special Zoology Reserve. In: Čalić, D., Roglić, J. (eds), Zbornik radova III. znanstvenog sabora Slavonije i Baranje 2. Jugoslavenska akademija znanosti i umjetnosti, Zavod za znanstveni rad Osijek, Osijek, 1021-1026. (in Croatian)

Mikuska, J. (1983b): A contribution to the understand of the fish fauna of the Kopački Rit Special Zoology Reserve. Anali Zavoda Jugoslavenske akademije, 2, 299-308. (in Croatian)

Mikuska, J. (1984): Changes to the fish community of Kopački Rit and the Danube River with regard to pollution. Čalić D, Berbe $Ð$ (eds), Zbornik radova IV. znanstvenog sabora Slavonije i Baranje 1. Jugoslavenska akademija znanosti i umjetnosti, Zavod za znanstveni rad Osijek, Osijek, 647-657. (in Croatian)

Mikuska, J., Mamić, M. (1984): Diet of the great cormorant Phalacrocorax carbo $(L, 1758)$ in Kopački Rit. Anali Zavoda za znanstveni i umjetnički rad u Osijeku, 3, 223230. (in Croatian)

Miladinović, Ž., Guzina, N., Vuković, T., Selimić, E. (1971): Agglutination of the erythrocytes of Paraphoxinus alepidotus, P. metohiensis and P. adspersus with the serums of trout, warmblood animals and humans. Ichthyologia, 3, 1, 49-55. (in Croatian)

Miller, P. J. (1990): The endurance of endemism: the Mediterranean freshwater gobies and their prospect for survival. Journal of Fish Biology, 37, 145-156.

Miller, P. J. (2004a): Proterorhinus marmoratus (Pallas, 1814). In: Miller, P. J. (ed), The Freshwater Fishes of Europe. Gobiidae 2. Vol 8/II. AULA-Verlag, Wiebelsheim, 72-93.

Miller, P. J. (2004b): Pomatoschistus canestrinii (Ninni, 1883). In: Miller, P. J. (ed), The Freshwater Fishes of Europe. Gobiidae 2. Vol 8/II. AULA-Verlag, Wiebelsheim, 284-292.

Miller, P. J. (2004c): Knipowitschia croatica Mrakovčić, Kerovec, Misetic and Schneider, 1994. In: Miller, P. J. (ed), The Freshwater Fishes of Europe. Gobiidae 2. Vol 8/II. AULA-Verlag, Wiebelsheim, 365-369.

Miller, P. J. (2004d): Knipowitschia panizzae (Verga, 1841). In: Miller, P. J. (ed), The Freshwater Fishes of Europe. Gobiidae 2. Vol 8/II. AULA-Verlag, Wiebelsheim, 405414.

Miller, P. J. (2004e): Knipowitschia punctatissima (Canestrini, 1864). In: Miller, P. J. (ed), The Freshwater Fishes of Europe. Gobiidae 2. Vol 8/II. AULA-Verlag, Wiebelsheim, 415-422.

Miller, P. J. (2004f): Padogobius bonelli (Bonaparte, 1846). In: Miller, P. J. (ed), The Freshwater Fishes of Europe. Gobiidae 2. Vol 8/II. AULA-Verlag, Wiebelsheim, 37-55. 
Miller, P. J., Šanda, R. (2008): A new West Balkanian sandgoby (Teleostei: Gobiidae). Journal of Fish Biology, 72, 259-270.

Miller, P. J. (2009): A west Balcanian freshwater gobiid fish, Knipowitschia mrakovcici sp. nov (Teleostei: Gobiidae). Journal of Fish Biology, 74, 1499-1509.

Miočić-Stošić, J., Kovačević, M. (2013): Research on the ichthyofauna of the upper course of the Zrmanja River. In: Basrek, L., Đud, L. (ed), Zbornik radova projekta "Istraživanje bioraznolikosti područja rijeke Zrmanje 2010“. Udruga studenata biologije - BIUS, Zagreb, 206218. (in Croatian)

Miotti, E. (2002): Pesci e biotopi quasi nostrani. Viaggio di un aquariofillo nell'ex Jugoslavia. Aquarium, 2, 58-65. (in Italian)

Mišetić, S., Mrakovčić, M., Šurmanović, D. (1989): Keeping of the trophic level of the Tribalj reservoir using biological regulators. Zbornik radova sa savjetovanja o ribarstvu na hidroakumulacijama, Mostar, 19-28. (in Croatian)

Mišković, Z. (1973): The catfish gets it - a monster. Ribarski list, 4, 112. (in Croatian)

Modrušan, Z., Teskeredžić, E. (1985): Mullets from Krka River estuary. Ichthyologia, 5, 1, 37-45.

Mojsisovics, A. E. (1883): Zur Fauna von Bellye und Darda. Mitteilungen des naturwissenschaftlichen Vereines für Steiermark, 19, 103-194. (in German)

Mojsisovics, A. E. (1884): Zur Fauna von Bellye und Därda (II. Theil.). Mitteilugen des naturwissenschaftlichen Vereines für Steiermark, 20, 122-170. (in German)

Mojsisovics, A. E. (1887): Zoologische Übersicht der österreichisch-ungarischen Monarchie. Druck und Verlag der Kaiserlich-Königlichen Hof und Staatsdruckerei, Wien, 249-328. (in German)

Mojsisovics, A. E. (1889): Zoogeographische Notizen über Süd-Ungarn aus den Jahren 1886-1888. Zugleich ein III. Nachtrag zur „Fauna von Béllye und Dárda“. Mitteilungen der Naturwissenschaftlichen Vereines für Steiermark, 25, 233-269. (in German)

Mojsisovics, A. E. (1897): Das Tierleben der österreichischungarischen Tiefebenen. Biologische und thiergeographische Skizzen und Bilder. Hölder, Wien, 334 pp. (in German)

Morović, D. (1947): Fisheries in the lower Neretva. Ribarstvo Jugoslavije, 2, 4, 37-38. (in German)

Morović, D. (1948): Annual catch trends of eels and mullets in the lower Neretva. Ribarstvo Jugoslavije, 3, 9-10, 83-86. (in Croatian)

Morović, D. (1955): Some observations on the length and weight of eels in the Neretva. Ribarstvo Jugoslavije, 10, 2, 28-30. (in Croatian)

Morović, D. (1956a): Adriatic mullets. Ribarstvo Jugoslavije, 11, 3-4, 65-69. (in Croatian)

Morović, D. (1956b): Adriatic mullets. Ribarstvo Jugoslavije, 11, 5, 93-98. (in Croatian)

Morović, D. (1956c): Adriatic mullets. Ribarstvo Jugoslavije,
11, 6, 111-114. (in Croatian)

Morović, D. (1958): Twaite shad, Clupea finta CUV. Ribarstvo Jugoslavije, 13, 5, 93-96. (in Croatian)

Morović, D. (1961): On the experimental research work in the Neretva Delta to improve lagoon fisheries and shellfish breeding. Ribarstvo Jugoslavije, 16, 6, 142146. (in Croatian)

Morović, D. (1962): Another contribution to understanding the issues of Vrana Lake. Morsko Ribarstvo, 14, 7-8, 2224. (in Croatian)

Morović, D. (1963): Distribution of the mullets along the eastern Adriatic coast. Ribarstvo Jugoslavije, 18, 4, 96102. (in Croatian)

Morović, D. (1964a): Vrana Lake. Priroda, 51, 7, 208-213. (in Croatian)

Morović, D. (1964b): Fisheries and biological issues in Vrana Lake. Ribarstvo Jugoslavije, 19, 4, 94-101. (in Croatian)

Morović, D. (1965): Some data on lampreys. Ribarstvo Jugoslavije, 20, 5, 99-102. (in Croatian)

Morović, D. (1966): Notes on the eel. Ribarstvo Jugoslavije, 21, 5, 98-101. (in Croatian)

Morović, D. (1967): Annual catch trends of mullets and eels in Vrana Lake. Morsko Ribarstvo, 19, 7-8, 127-130. (in Croatian)

Morović, D. (1970): Quelques observations sur l'anguille, Anguilla anguilla L., de la côte orientale de l'Adriatique. Acta Adriatica, 27, 1-4. (in French)

Morović, D. (1972): Declining numbers of eels in the lower Neretva area. Morsko Ribarstvo, 24, 3, 111-113. (in Croatian)

Morović, D. (1976): On the possibilities of developing lagoon fisheries in the area of the Old River (Metković). Ribarstvo Jugoslavije, 31, 1, 6-13. (in Croatian)

Morović, D. (1978): Harpoons in the Neretva Valley. Morsko Ribarstvo, 30, 4, 191. (in Croatian)

Mrakovčić, M. (1990): Is there hope for the survival of the softmouth trout?. Ekološki glasnik, 1-2, 17-19. (in Croatian)

Mrakovčić, M. (1992): Succession of ichthyofauna in the Varaždin reservoir. PhD thesis, Faculty of Science, University of Zagreb. (in Croatian with English abstract)

Mrakovčić, M. (1998): Animal life in the Nereva River Valley, with an emphasis on fisheries. Časopis za književnost i znanost, 9, 253-259. (in Croatian)

Mrakovčić, M., Brigić, A., Buj, I., Ćaleta, M., Mustafić, P., Zanella, D. (2006): Red Book of Freshwater Fishes of Croatia. Ministarstvo kulture, Državni zavod za zaštitu prirode, Zagreb, 253pp. (in Croatian with English abstract)

Mrakovčić, M., Ćaleta, M. (2011): Endemic fishes of the Cetina Basin. Prirodoslovlje: časopis Odjela za prirodoslovlje i matematiku Matice hrvatske, 11, 3-18. (in Croatian with English abstract)

Mrakovčić, M., Duplić, A., Mustafić, P., Marčić, Z. (2008): 
Conservation status of the genus Cobitis and related genera in Croatia. Folia Zoologica, 57, 35-41.

Mrakovčić, M., Kerovec, M. (1990): Umbra krameri. Ekološki glasnik, 5-6, 68-69. (in Croatian)

Mrakovčić, M., Kerovec, M., Mišetić, S., Schneider, D. (1996a): Description of Knipowitschia punctatissima croatica, (Pisces: Gobiidae), a new freshwater goby from Dalmatia, Croatia. In: Kirchhofer, A., Hefti, D. (eds), Conservation of endangered freshwater fish in Europe, Birkhäuser Verlag, Basel, Switzerland, 311-319.

Mrakovčić, M., Kerovec, M., Mišetić, S., Schneider, D., Tomašković, N., Šurmanović, D. (1996b): Ichthyofauna of the Drava river (Croatia). Limnologische Berichte Donau, 1, 345-348.

Mrakovčić, M., Mišetić, S. (1990a): Significance and characteristics of the ichthyofauna of the Krka River. In: Kerovec, M. (ed), Problemi zaštite Nacionalnog parka Krka, Knjiga 2. Hrvatsko ekološko društvo, Zagreb, 271282. (in Croatian)

Mrakovčić, M., Mišetić, S. (1990b): Status, distribution and conservation of the salmonid Salmothymus obtusirostris (Heckel) and the cyprinid Aulopyge hugeli (Heckel) in Yugoslavia. Journal of Fish Biology, 31, 241242.

Mrakovčić, M., Mišetić, S., Povž, M. (1995): Status of freshwater fish in Croatian Adriatic river systems. Biological Conservation, 72, 179-185.

Mrakovčić, M., Mustafić, P., Ćaleta, M., Zanella, D., Radić, I. (2002): Charasteristics of the fish communities in Lonjsko Polje Nature Park. Bilten Parka prirode Lonjsko polje, 4, 1-56. (in Croatian)

Mrakovčić, M., Popović, J., Šurmanović, D. (1989): Ichthyofauna of Lake Lokve, Gorski Kotar, Yugoslavia. Periodicum Biologorum, 91, 1, 109.

Mrakovčić, M., Schneider, D., Kerovec, M. (1994): Freshwater gobies of Croatia. Periodicum Biologorum, 96, 2, 441-443.

Mrakovčić, M., Schneider, D., Kerovec, M., Mustafić, P., Bukvić, I. (2000b): Change of native fish community after closure of the water reservoir on river Butoniga (Istria, Croatia). In: Collares-Pereira, M. J., Coelho, M., Cowx, I. (eds), Freshwater Fish Conservation: Options for the Future. Faculdade de Ciencias de Lisboa, Lisboa, $67 p p$.

Mrakovčić, M., Schneider, D., Mišetić, S., Šurmanović, D. (2000a): Ichthyofauna of the Baćina Lakes. In: Kerovec, M., Durbešić, P. (eds), Prirodoslovna istraživanja biokovskog područja. Hrvatsko ekološko društvo, Zagreb, 203-212. (in Croatian)

Mrakovčić, M., Schneider, D., Mustafić, P., Kerovec, M. (2000c): Status of genus Cobitis and related species in Croatia. Folia Zoologica, 49, 113-116.

Mršić, V. (1932a): On the Dobra. In: Thaller, Z. (ed), Vode i ribe Jugoslavije u slici i riječi. Tipografija, Zagreb, 158162. (in Croatian)

Mršić, V. (1932b): Trout of the Jadro River. In: Thaller, Z. (ed), Vode i ribe Jugoslavije u slici i riječi. Tipografija, Zagreb, 71-72. (in Croatian)

Mršić, V. (1935a): Experiences in the domestification of rainbow trout in Yugoslavia. Ribarski vjesnik, 13, 3, 4954. (in Croatian)

Mršić, V. (1935b): Experiences in the domestification of rainbow trout in Yugoslavia. Ribarski vjesnik,13, 3-4, 91-94. (in Croatian)

Mršić, V. (1935c): Experiences in the domestification of rainbow trout in Yugoslavia. Ribarski vjesnik, 13, 7, 154157. (in Croatian)

Mršić, V. (1935d): Experiences in the domestification of rainbow trout in Yugoslavia. Ribarski vjesnik, 13, 8, 181190. (in Croatian)

Mršić, V. (1938): Difference forms of the species Trutta obtusirostris in the Jadro River. Ribarstvo, 1, 1-2, 7-10. (in Croatian)

Muhamedagić, S., Gjøen, H. M., Vegara, M. (2008): Salmonids of the Neretva river basin - present state and suggested sustainable selection programme to protect and strengthen salmonid populations. Proceedings of the EIFAC symposium on iteractions between social, economic and ecologicak objectives on inland commercial and recriational fisheries and aquaculture. FAO, Turkey, 224-275.

Muhamedagić, S., Habibović, E. (2013): The State and Perspective of Danube huchen (Hucho hucho) in Bosnia and Herzegovina. Archives of Polish Fisheries, 21, 155160.

Munjko, I. (1972): On the mass death of fishes in the Sava River. Priroda, 61, 7, 205-206. (in Croatian)

Munjko, I. (1975): On the mass death of fishes in the Sava River from Zagreb to Sisak. Ribarstvo Jugoslavije, 30, 6, 126-129. (in Croatian)

Munjko, I. (1978): Importance of testing surface waters in the Lonje River basin. Ribarstvo Jugoslavije, 33, 2, 3037. (in Croatian)

Mustafić, P. (2001): Ichthyofauna of different habitat types in the central course of the Drava River. Master's thesis, Faculty of Science, University of Zagreb. (in Croatian with English abstract)

Mustafić, P. (2005): Index of biotic integrity of the fish community in the large Croatian rivers. PhD thesis, Faculty of Science, University of Zagreb. (in Croatian with English abstract)

Mustafić, P., Buj, I., Opašić, M., Zanella, D., Marčić, Z., Ćaleta, M., Šanda, R., Horvatić, S., Mrakovčić, M. (2016): Morphological comparison of Delminichthys ghetaldii (Steindachner, 1882), D. adspersus (Heckel, 1843), D. jadovensis (Zupančič \& Bogutskaya, 2002) and D. krbavensis (Zupančič \& Bogutskaya, 2002), endemic species of the Dinaric karst, Croatia. Journal of Applied Ichthyology, 33, 256-262.

Mustafić, P., Ćaleta, M., Mrakovčić, M., Buj, I., Zanella, D., Mišetić, S. (2005): Distribution and status of the genus Gobio in Croatia. Folia Zoologica, 54, 81-84. 
Mustafić, P., Marčić, Z., Duplić, A., Mrakovčić, M., Ćaleta, M., Zanella, D., Buj, I., Podnar, M., Dolenec, Z. (2008): A new loach species of the genus Cobitis in Croatia. Folia Zoologica, 57, 4-9.

Mustafić, P., Marčić, Z., Zanella, D., Ćaleta, M., Buj, I., Horvatić, S., Mrakovčić, M. (2017): Ichthyofauna of the Vrana Lake Nature park. In: Rubinić, J. (ed), Managing lakes and reservoirs in Croatia - process, protection and evaluation. Scientific and technical conference with international participation. 4-6 May 2017, Biograd, 227-235.

Mustafić, P., Mrakovčić, M. (2017): Biodiversity status of and threats to freshwater fish of Croatia and Eastern Adriatic Countries. In: Berillis, P. (ed), Trends in Fisheries and Aquatic Animal Health, Bentham Science Publishers - Sjarjah, UAE, 23-41.

Mustafić, P., Mrakovčić, M., Ćaleta, M., Radić, I., Zanella, D., Mihaljević, Z., Ternjej, I. (2003): Loaches in a long term study of the Drava river in Croatia. Folia Biologica (Krakow), 51, 143-146.

Mušović, A., Đug, S., Pojskić, N., Kalamujić Stroil, B., Vesnić, A., Škrijelj, R. (2018): Status of endangered fish species Aulopyge huegelii Heckel, 1843 (Teleostei: Cyprinidae) in the Buško Blato reservoir, Bosnia and Herzegovina. Iranian Journal of Ichthyology, 5, 3, 212-231.

Nardo, G. D. (1847): Sinonimia moderna delle specie registrate nell' opera intitolata: descrizione de' crostacei, de' testacei e de' pesci che abitano le lagune e Golfo Veneto rappresentati in figure, a chiaro-scuro ed a colori dall'abate Stefano Chiereghini Ven. Clodiense. Antonelli. Nell'I.R. Priv. Stabilimento Antonelli, Venezia, $\mathrm{xi}+128 \mathrm{pp}$. (in Italian)

Naseka, A. M., Bogutskaya, N. G., Bănărescu, P. M. (1999): Gobio alnipinnatus Lukasch, 1933. In: Bănărescu, P. M. (ed), The Freshwater Fishes of Europe. Cyprinidae 2/l. Vol. 5/I. AULA-Verlag, Wiebelsheim, 37-68pp.

Nedić, Z., Skenderović, I., Riđanović, S. (2014a): Skin ectoparasites of fish from the lower flow of the Sava River. Veterinaria, 63, 1-4, 45-53.

Nedić, Z., Begović, S., Dogan, M., Hadžiahmetović Jurida, E., Ferizbegović, J., Terzić, R. (2014b): Decreasing of biodiversity of fish populations from the Sava River in Bosnia and Herzegovina. Basic Research Journal of Agricultural Science and Review, 3, 5, 35-40.

Nedić, Z., Skenderović, I., Adrović, A. (2018): Study of some ectoparasites of fishes from the Sava River as part of water management in Bosnia and Herzegovina. TEM Journal, 7, 2, 391-397.

Nejedli, S., Kozarić, Z., Katavić, I., Žura Žaja, I., Tlak Gajger, I. (2018): Morphology of the epaxial musculature and osteological development of the early developmental stages of softmouth trout (Salmothymus obtusirostris, Heckel, 1851). Veterinarski arhiv, 88, 1, 89-100.

Nežić, E. (1938): The mosquito fish in the battle against malaria. Ribarstvo, 1, 3-6, 62-66. (in Croatian)

Ninni, A. P. (1883): Nuova specie di Gobius. Atti della
Societa Veneto-Trentina di Scienza Naturali in Padova, 8, 276-279. (in Italian)

Ninni, E. (1930): Sopra la Trutta adriatica (Kolomb.). Bulletin de la Société Zoologique de France, 55, 446451. (in Italian)

Nocita, A., Vanni, S. (1997): Cataloghi del museo di storia naturale dell'universita di Firenze - sezione di zoologia "La Specola“. XIX Actynopterigii Cypriniformes. Atti della Società Toscana di Scienze Naturali, Memorie, Serie B, 104, 61-69. (in Italian)

Nocita, A., Vanni, S. (1999): Cataloghi del museo di storia naturale dell'universita di Firenze - sezione di zoologia „La Specola“. XIX Actynopterigii Cypriniformes. Atti della Società Toscana di Scienze Naturali, Memorie, Serie B, 106, 115-130. (in Italian)

Ocvirk, A. (1984): Ichthyological reserches of the Gacka river and growth dinamics of brown trout (Salmo trutta m. fario L.). Ichthyos, 1, 7-13.

Ocvirk, J. (1985): Growth dynamics of the pike (Esox lucius Linnaeus 1758) in Vrana Lake, Island of Cres. Ichthyos, 2, 11-17. (in Croatian)

Ocvirk, J. (1992): Growth dynamics of the pike (Esox lucius Linnaeus 1758) in Vrana Lake, Island of Cres. In: Sokolić, J. (ed), Biologija Cresa i Lošinja. Fond za kulturu CresLošinj, Katedra Čakavskog sabora Cres-Lošinj, Mali Lošinj-Rijeka, 192-199. (in Croatian)

Odak, T. (2004): Molecular-biological characteristic of endemic softmuzzled trout (Salmothymus obtusirostris salonitana). Master's thesis, Faculty of Agriculture, University of Zagreb. (in Croatian with English abstract)

Odak, T., Treer, T., Aničić, I., Safner, R., Piria, M. (2002): The use of molecular methods in fisheries. Ribarstvo, 60, 3, 116-126. (in Croatian with English abstract)

Odak, T., Treer, T., Safner, R., Aničić, I., Piria, M. (2003): Soft-muzzled trout (Salmothymus obtusirostris salonitana) in the Žrnovnica River. In: Žimbrek, T. (ed), Zbornik radova s 37. znanstvenog skupa hrvatskih agronoma. 37. znanstveni skup hrvatskih agronoma Opatija (Hrvatska), February 2003. Kuliš d.o.o, Zagreb, 429-432.

Oikonomou, A., Leprieur, F., Leonardos, I. D. (2014): Biogeography of freshwater fishes of the Balkan Peninsula. Hydrobiologia, 738, 1, 205-220.

Oliva, O., Zanandrea, G. (1959): Posizione sistematica di una lampreda di Cilistovo (Cecoslovacchia). Doriana, 2, 98, 1-5. (in Italian)

Opačak, A., Florijančić, T., Horvat, D., Ozimec, S., Bodakoš, D. (2004): Diet spectrum of great cormorants (Phalacrocorax carbo sinensis L.) at the Donji Miholjac carp fishponds in eastern Croatia. European Journal of Wildlife Research, 50, 4, 173-178.

Ozimec R (2015): The oldest European fish in Croatia. Hrvatska vodoprivreda, 211, 108-113. (in Croatian)

Ozimec, S., Opačak, A., Jelkić, D., Florijančić, T., Bošković, I., Puškadija, Z., Majić, S. (2010): Comparison of the hydrobiological properties of selected fishing waters in 
Podravina. In: Marić, S., Lončarić, Z. (eds), 45. hrvatski i 5. međunarodni simpozij agronoma, Opatija (Hrvatska), February 2010. Poljoprivredni fakultet, Osijek, 983987. (in Croatian)

Paepke, H-J. (2002): Gasterosteus aculeatus Linnaeus, 1758. In: Bănărescu, P. M., Paepke, H-J. (eds), The Freshwater Fishes of Europe. Cyprinidae 2/III, Gasterosteidae. Vol 5/III. AULA-Verlag, Wiebelsheim, 209-256.

Palandačić, A., Bravničar, J., Zupančič, P., Šanda, R., Snoj, A. (2015): Molecular data suggest a multispecies complex of Phoxinus (Cyprinidae) in the Western Balkan Peninsula. Molecular Phylogenetics and Evolution, 92, 118-123.

Palandačić, A. (2012a): Molecular ecology of imotska gaovica (Delminichthys adspersus (Heckel, 1843)). PhD thesis, Biotechnical Faculty, University of Ljubljana. (in Croatian with English abstract)

Palandačić, A. (2012b): Mysterious minnow. Bilten slovenskih terenskih biologov in ljubiteljev narave, 1, 1, 19. (in Slovenian)

Palandačić, A., Matschiner, M., Zupančič, P., Snoj, A. (2012a): Fish migrate underground: the example of Delminichthys adspersus (Cyprinidae). Molecular Ecology, 21, 7, 1658-1672.

Palandačić, A., Bonacci, O., Snoj, A. (2012b): Molecular data as a possible tool for tracing groundwater flow in karst environment: example of Delminichthys adspersus in Dinaric karst system. Ecohydrology, 5, 6, 791-797.

Palandačić, A., Zupančić, P., Snoj, A. (2010): Revised classification of former genus Phoxinellus using nuclear DNA sequences. Biochemical systematics and Ecology, 38, 1069-1073.

Pallaoro, A. (1998): Pantan lagoons and surrounding areas. Radovan, 2, 2, 95-102. (in Croatian)

Parenzan, P. (1928): Il Lago d'Arsa. Histria Nobilissima, 1, 47-51. (in Italian)

Pavletić, J. (1954): A rare fish - the European mudminnow. Ribarstvo Jugoslavije, 9, 3, 62-64. (in Croatian)

Pavlica, M., Štambuk, A., Malović, L., Mladinić, M., Klobučar, G. I. V. (2011): DNA integrity of chub erythrocytes (Squalius cephalus L.) as an indicator of pollution-related genotoxicity in the River Sava. Environmental Monitoring and Assessment, 177, 1-4, 85-94.

Pavličević, J., Bogut, I., Glamuzina, B. (2010): Protection and improvement of the status of the endemic salmonids of the Neretva River: Dentex trout - Salmo dentex, Softmouth trout - Salmo obtusirostris, Sea trout - Salmo farioides and Marble trout - Salmo marmoratus. In: Glamuzina, B., Dulčić, J. (eds), Ribe i Ribarstvo, rijeke Neretve. Međunarodni znanstvenostručni skup "Ribe i Ribarstvo, rijeke Neretve: stanje i perspektive" Opuzen (Hrvatska), 2009. Sveučilište u Dubrovniku i Dubrovačko neretvanska županija,
Dubrovnik, 143-153. (in Croatian)

Pavličević, J., Glamuzina L, Conides, A., Savić, N., Rozić, I., Klaoudatos, D., Kazić, A., Glamuzina, B. (2016): Pikeperch, Sander lucioperca invasion in the Neretva River watershed (Bosnia and Herzegovina, Croatia) after alteration of river flow. River Research and Applications, 32, 967-974.

Pažur, K. (1964): Salmonid fish farming and the issues of feed base in Yugoslavia. Ribarstvo Jugoslavije, 19, 1, 1720. (in Croatian)

Pažur, K. (1965): Final farewell to the Dretulja and Mrežnica. Ribarski list, 1, 21-22. (in Croatian)

Pažur, K. (1968a): Stocking pike in the Lika River basin. Ribarstvo Jugoslavije, 23, 1, 25. (in Croatian)

Pažur, K. (1968b): Good catch of huchens in the Kupa and Dobra. Ribarstvo Jugoslavije, 23, 4, 96. (in Croatian)

Pažur, K. (1969a): Giant trout in Lokvarka Lake. Ribarski list, 1, 9-11. (in Croatian)

Pažur, K. (1969b): Giant trout in Lokvarka Lake. Ribarstvo Jugoslavije, 24, 2, 40-43. (in Croatian)

Pažur, K. (1969c): Researching our high elevation waters. Ribarski list, 3, 55-59. (in Croatian)

Pažur, K. (1970): Possibilities of developing fishing tourism in the area of the "Plitvice Lakes" National Park. Ribarstvo Jugoslavije, 25, 3, 66-69. (in Croatian)

Pažur, K. (1975): State and perspectives of fishing in the Ogulin municipality. Ribarstvo Jugoslavije, 30, 2, 38-39. (in Croatian)

Pažur, K. (1979): Problemwasser Gacka. Der Fliegenfischer, 30, 42-44. (in German)

Pažur, K. (1993): Fishing on the Una in the Kostajnica area. Ribarstvo, 48, 3, 111-112. (in Croatian)

Pažur, K. (1995): An unusual catch. Ribarstvo, 53, 2, 75. (in Croatian)

Pažur, K. (2005): Sports fishing handbook. Hrvatski športsko ribolovni savez, Zagreb, 137pp. (in Croatian)

Pažur, K., Habeković, D., Popović, J. (1982): Growth dynamics of huchen (Hucho hucho L. 1758) in the waters of SR Croatia. Ichthyologia, 14, 2, 161-169. (in Croatian)

Peheim, I. (1905): A rare catch. Lovačko-ribarski viestnik, 14, 45-46. (in Croatian)

Peheim, I. (1909): A rare catch. Lovačko-ribarski viestnik, 18, 47. (in Croatian)

Perdices, A., Bohlen, J., Doadrio, I. (2008): The molecular diversity of adriatic spined loaches (Teleostei, Cobitidae). Molecular Phylogenetics and Evolution, 46, 1, 382-390.

Perea, S., Böhme, M., Zupančič, P., Freyhof, J., Šanda, R., Özuluğ, M., Abdoli, A., Doadrio, I. (2010): Phylogenetic relationships and biogeographical patterns in CircumMediterranean subfamily Leuciscinae (Teleostei, Cyprinidae) inferred from both mitochondrial and nuclear data. BMC Evolutionary Biology, 10, 265, 1-27. Peričić, Š. (1993): Commerical situation of Dalmatia from 
1797 to 1848. Biblioteka znanstvenih djela, knjiga 65, Književni krug, Split, 176pp. (in Croatian)

Perović, F., Tvrtković, N. (2010): Preliminary overview of the lampreys and fishes of the Majdan and Žirovski streams. In: Bučar, M. (ed), Zrinska gora regionalni park prirode. Grad Petrinja, Sisačko-Moslavačka županija, Sveučilište u Zagrebu Učiteljski fakultet Odsjek Petrinja, Matica hrvatska ogranak Petrinja, Petrinja, 208-211. (in Croatian)

Perugia, A. (1881): Elenco dei pesci dell' Adriatico. Ulrico Hoepli, Milano, 60pp. (in Italian)

Pešić, V. (1955): Four fishing districts. Ribarski list, 4, 7881. (in Croatian)

Pešić, V. (1961): Fish of the Adriatic basin. Ribarski list, 6, 118-120. (in Croatian)

Petermann, R. E. (1899): Führer Durch Dalmatien. Hölder, Wienna, 602pp. (in German)

Petrinec, Z., Nejedli, S., Kužir, S., Opačak, A. (2005): Mucosubstances of the digestive tract mucosa in northern pike (Esox lucius L.) and european catfish (Silurus glanis L.). Veterinarski arhiv, 4, 317-327.

Pilicar, F. (1959): Translocated fish. Ribarski list, 3, 61. (in Croatian)

Piller, M., Mitterpacher, L. (1783): Iter per Poseganam Sclavoniae provincism mensibus Junio et Julio anno 1782 susceptum. Typis Regiae Universitatis, Budae, 147pp. (in Latin)

Piria, M. (2007): Ecological and biological diet features of cyprinid fish from the Sava River. PhD thesis, Faculty of Agriculture, University of Zagreb. (in Croatian with English abstract)

Piria, M., Jakšić, G., Jakovlić, I., Treer, T. (2016a): Dietary habits of invasive Ponto-Caspian gobies in the Croatian part of the Danube River basin and their potential impact on benthic fish communities. Science of the Total Environment, 540, 386-395.

Piria, M., Matulić, D., Šprem, N., Reljanović, M., Novosel, H., Buničić, S., Kranjec, I., Drašković, A., Duduković, D., Medvidović, I., Ređić, J. (2009): Ichthyofauna of the lower part of Bliznec stream. Ribarstvo, 4, 145-152. (in Croatian with English abstract)

Piria, M., Milošević, D., Šprem, N., Mrdak, D., Tomljanović, T., Matulić, D., Treer, T. (2016): Condition of European eel from the Adriatic basin of Croatia and Montenegro. In: Pospišil, M., Vnučec, I. (eds), Proceedings of $51^{\text {st }}$ Croatian and $11^{\text {th }}$ International Symposium of Agriculture. $51^{\text {st }}$ Croatian and $11^{\text {th }}$ International Symposium of Agriculture, Opatija (Croatia), February, 2016. Faculty of Agriculture, University of Zagreb, Zagreb, 269-273.

Piria, M., Povž, M., Vilizzi. L., Zanella, D., Simonović, P., Copp, H. G. (2016b): Risk screening of non-native freshwater fishes in Croatia and Slovenia using the Fish Invasiveness Screening Kit. Fisheries Management and Ecology, 23, 1, 21-31.

Piria, M., Simonović, P., Nikolić, V., Ristovska, M.,
Apostolou, A., Adrović, A., Povž, M., Zanella, D., Mrdak, D., Milošević, D., Vardakas, L., Koutsikos, N., Kalogianni, E., Gregori, A., Kostov, V., Škrijelj, R., Korro, K., Bakiu, R., Tarkan, A. S., Joy, M. K. (2018): Alien freshwater fish species in the Balkans - vectors and pathways of introduction. Fish and Fisheries, 19, 1, 138-169.

Piria, M., Svjetličić, S., Poljak, A., Jakovlić, I. (2017): Diet composition of Topmouth gudgeon Pumpkinseed sunfish and Black bullhead catfish and their invasive potential in Croatia. In: Vila, S., Antunović, Z. (eds), Proceedings of $52^{\text {nd }}$ Croatian and $12^{\text {th }}$ International symposium of Agriculture. $52^{\text {nd }}$ Croatian and $12^{\text {th }}$ International symposium of Agriculture, Opatija (Croatia), February, 2017. Faculty of Agriculture, Josip Juraj Strossmayer University of Osijek, Osijek, 428-432.

Piria, M., Šprem, N., Jakovlić, I., Tomljanović, T., Matulić, D., Treer, T., Aničić, I., Safner, R. (2011c): First record of round goby, Neogobius melanostomus (Pallas, 1814) in the River Sava, Croatia. Aquatic Invasions, 6, 1, 153157.

Piria, M., Šprem, N., Tomljanović, T., Slišković, M., Jelić Mrčelić, G., Treer, T. (2014): Length-weight relationships of european eel Anguilla anguilla (Linnaeus, 1758) from six karst catchments of the Adriatic basin, Croatia. Croatian Journal of Fisheries, 72, 1, 32-35.

Piria, M., Tomljanović, T., Treer, T., Safner, R., Aničić, I., Matulić, D., Vilizzi, L. (2016c): The common carp Cyprinus carpio in Croatia (Danube and Adriatic basins) a historical review. Aquaculture International, 24, 6, 1527-1541.

Piria, M., Treer, T., Aničić, I., Safner, R., Odak, T. (2004): The review on the natural diet of five cyprinid fish species. 39. In: Žimbrek, T. (ed), Proceedings from 39. Croatian Symposium on Agriculture with International Participation. 39. Croatian Symposium on Agriculture with International Participation, Opatija (Croatia), February, 2005. Faculty of Agriculture, University of Zagreb, 579-582.

Piria, M., Treer, T., Aničić, I., Safner, R., Odak, T. (2005b): The natural diet of five cyprinid fish species. Poljoprivredna znanstvena smotra, 1, 21-28.

Piria, M., Treer, T., Aničić, I., Tomljanović, T., Safner, R. (2007): Natural diet of barbel Barbus peloponnesius (Valenciennes, 1842) from the Sava River. Ribarstvo, 1, 1-14. (in Croatian with English abstract)

Piria, M., Treer, T., Ivanković, P., Tomljanović, T., Aničić, I., Safner, R., Matulić, D., Šprem, N. (2012): Threatened fishes of the world: Squalius svallize (Heckel \& Kner, 1858) (Cyprinidae). Ribarstvo, 70, 3, 143-146.

Piria, M., Treer, T., Tomljanović, T., Šprem, N., Matulić, D., Aničić, I., Safner, R. (2011a): First record of monkey goby, Neogobius fluviatilis (Pallas, 1814) in the barbel zone of the River Sava, Croatia. Journal of Applied Ichthyology, 27, 1383-1384.

Piria, M., Vrbanec, D., Đurđević, G. (2011a): Von Bertalanffy growth curve of the Rudd (Scardinius 
erythrophthalmus L.) from the Lake Ključić Brdo, central Croatia. Ribarstvo, 69, 4, 127-136. (in Croatian with English abstract)

Piria, M., Odak, T., Treer, T., Aničić, I., Safner, R. (2005a): Diet of Chub and Barbel in the Sava River. Radovi Poljoprivredno-prehrambenog fakulteta, Univerziteta u Sarajevu 56, 119-127. (in Croatian)

Pirogovskiĭ, M. I., Sokolov, L. I., Vasil'ev, V. P. (1989): Huso huso (Linnaeus, 1758). In: Holčík, J. (ed), The Freshwater Fishes of Europe. Vol 1, Part II, General introduction to fishes, Acipenseriformes. AULA-Verlag, Wiesbaden, 156-200.

Pizzul, E., Borme, D., Cassetti, P., Amirante, G. A., Battistella, S. (2002): Comparison between two populations of bleak Alburnus alburnus (L.) (Osteichthyes, Cyprinidae) of the Friulan low plane (north-eastern Italy) and of the Danube basin (north-eastern Croatia) on morphological and genetical basis. Ichthyos, 1, 9-20.

Plančić, J. (1920): A study of the fisheries and commercial opportunites of the Danube. Lovačko-Ribarski vjesnik, 29, 39-49. (in Croatian)

Plančić, J. (1923a): Fish of the Sava River. Lovačko-Ribarski vjesnik, 32, 90-99. (in Croatian)

Plančić, J. (1923b): Fish of the Sava River. Lovačko-Ribarski vjesnik, 32, 74-79. (in Croatian)

Plančić, J. (1935a): Report on the work of the Zagreb Fishing Society for 1934. Ribarski vjesnik, 13, 1, 13-17. (in Croatian)

Plančić, J. (1935b): Our fish ponds and their task. Ribarski vjesnik, 13, 7, 162-166. (in Croatian)

Plančić, J. (1938): Stocking the Plitvice Lakes with whitefish. Ribarstvo, 1-2, 5-7. (in Croatian)

Plančić, J. (1946): Newly stocked fish in the Lika River. Ribarstvo Jugoslavije, 1, 3, 31. (in Croatian)

Plančić, J. (1948): Commercial significance of Vrana Lake. Ribarstvo Jugoslavije, 3, 5, 35-38. (in Croatian)

Plančić, J. (1949): Importance of eels in the human diet. Ribarski kalendar 1949, Riba export-import, Zagreb, 157pp. (in Croatian)

Plančić, J. (1950): Stocking the Visovac Lake, Mišovica River and Imotski Lake with carp. Slatkovodno Ribarstvo Jugoslavije, 5, 8, 160-161. (in Croatian)

Plančić, J. (1952a): Dieoffs of eels in Vrana Lake. Ribarstvo Jugoslavije, 7, 1-2, 16-17. (in Croatian)

Plančić, J. (1952b): Some data for determining the minimum measures of grayling. Ribarstvo Jugoslavije, 7, 4, 52-53. (in Croatian)

Plančić, J. (1952c): Mirna River - possibilities of its use for fisheries. Ribarstvo Jugoslavije, 7, 6, 89-92. (in Croatian)

Plančić, J. (1952d): Catching a marked sterlet. Ribarstvo Jugoslavije, 7, 8, 147. (in Croatian)

Plančić, J. (1952e): Experimental trawl fishery in the Danube. Ribarstvo Jugoslavije, 7, 1-2, 9-10. (in Croatian)

Plančić, J. (1953a): Importance of mullet in our brackish and freshwaters. Morsko Ribarstvo, 5, 1-2, 21-22. (in
Croatian)

Plančić, J. (1953b): A brief overview of the freshwater fisheries of Yugoslavia. Ribarski list, 6, 89-92. (in Croatian)

Plančić, J. (1955): The problem of fisheries on Vrana Lake. Morsko Ribarstvo, 7, 12, 331-334. (in Croatian)

Plančić, J. (1956a): Who is to blame for the poor situation on Vrana Lake?. Morsko Ribarstvo, 8, 5, 150. (in Croatian)

Plančić, J. (1956b): Construction of a dam on the Mrežnica River near Ogulin and the consequences on fisheries. Ribarstvo Jugoslavije, 11, 3-4, 47-49. (in Croatian)

Plančić, J. (1956c): Our salmonid species and their spawning. Ribarski list, 2-3, 27-32. (in Croatian)

Plančić, J. (1967): Prussian carp, Carassius auratus gibelio Bloch, a new species for our ichthyofauna. Ribarstvo Jugoslavije, 22, 6, 155-156. (in Croatian)

Plančić, J., Stanić-Mayer, D., Marko, S. (1953): A contribution to the fisheries and biological research of the Gacka River. Ribarstvo Jugoslavije, 8, 1, 1-9. (in Croatian)

Podrug, M., Raspor, B. (2009): Protein and metal concentrations in two fractions of hepatic cytosol of the European chub (Squalius cephalus L.). Chemosphere, 75, 843-849.

Podrug, M., Raspor, B., Erk, M., Dragun, Z. (2009): Seasonal variation of the metal $(\mathrm{Zn}, \mathrm{Fe}, \mathrm{Mn})$ and metallothionein concentrations in the liver cytosol of the European chub (Squalius cephalus L.). Environmental Monitoring and Assessment, 157, 1-10.

Pofuk, M., Zanella, D., Piria, M. (2017): An overview of the translocated native and non-native fish species in Croatia: pathways, impacts and management. Management of Biological Invasions, 8 (3): 425-435.

Pogrmilović, P. (1972): Damages caused by catastrophic floods on ponds. Ribarstvo Jugoslavije, 27, 5, 113-116. (in Croatian)

Polačik, M., Janáč, M., Trichkova, T., Vassilev, M., Keckeis, H., Jurajda, P. (2008): The distribution and abundance of the Neogobius fishes in their native range (Bulgaria) with notes on the non-native range in the Danube River. Large Rivers, 18, 193-208.

Poljak, J. (1924): Velebit. Priroda, 14, 9-10, 159-160. (in Croatian)

Pompe Gotal, J. (1984): Level of mercury contamination in animal tissues from the ecological region of Crna Mlaka. PhD thesis, Faculty of Science, University of Zagreb. (in Croatian)

Popović, J. (1991): The estimate and the analysis of mortality and survival of pike (Esox lucius L. 1758) at Čambina area next to the Drava River. Ribarstvo Jugoslavije, 46, 3-6, 47-52. (in Croatian with English abstract)

Popović, J. (1994): Significance of the scales of the Illyrian chub (Leuciscus illyricus Heckel et Kner, 1858) of the Cetina River. Ribarstvo, 52, 3, 1-11. (in Croatian) 
Popović, J. (2010): Management of fishing waters. Hrvatski športsko ribolovni savez, Zagreb, 215pp. (in Croatian)

Popović, J., Fašaić, K. (1982): Seasonal changes to allometric growth in brown trout (Salmo trutta $\mathrm{m}$. fario L.) from the Zrmanja River. Ribarstvo Jugoslavije, 37, 3, 49-53. (in Croatian)

Popović, J., Fašaić, K. (1986): Qualitative and quantitative characteristics of the ichthyofauna in the Virovitica area. Ekologija, 1, 45-52. (in Croatian)

Popović, J., Fašaić, K., Homen, Z. (1984): Comparative research of the length-weight relationship in eels (Anguilla anguilla L. 1758) from two different ecosystems. Ichthyologia, 16, 1-2, 29-41. (in Croatian)

Popović, J., Habeković, D. (1981): Length-weight relationship of the Illyrian chub (Leuciscus illyricus, Heckel et Kner, 1858) from the Peruča reservoir. Ichthyologia, 13, 1, 73-80. (in Croatian)

Popović, J., Šurmanović, D., Mišetić, S., Tomašković, N. (1992): Natural diet of the Illyrian chub Leuciscus illyricus (Heckel et Kner 1858) from the Cetina River. Poljoprivredna znanstvena smotra, 3-4, 425-434. (in Croatian)

Popović, K. (1935): One fishing experience. Ribarski vjesnik, 13, 7, 171-173. (in Croatian)

Porečnik, R. (1958): The hunt for eels. Ribič, 10, 295-296. (in Slovenian)

Povž, M. (1982): Carassius auratus gibelio (Bloch) 1783 (Pisces): in Slovenia. Biološki vestnik, 1, 121-126. (in Slovenian)

Povž, M. (1983): The change of the areal of two species from genus Chondrostoma (Pisces) in Slovenia. Biološki vestnik, 31 (1): 45-52.

Povž, M. (1984): The distribution range of the European mudminnow Walbaum, 1772 (Osteichthyes) in Slovenia. Ichthyologia, 16, 1-2, 43-48. (in Slovenian)

Povž, M. (1995): Discovery, distribution, and conservation of mudminnow Umbra krameri Walbaum, 1792 in Slovenia. Annalen des Naturhistorischen Museums in Wien, 97, B, 478-485.

Povž, M. (1999): Biological and ecological caracteristics of Rutilus pigus virgo (Heckel, 1852) population. PhD thesis, Faculty of Science, University of Zagreb. (in Croatian with English abstract)

Povž, M. (2002): Fish of the Dragonja River. Varstvo narave, 19, 69-75. (in Slovenian)

Povž, M. (2011): Distribution and conservation status of the lampreys in Slovenia. Bulletin Lampetra, 7, 84-91.

Povž, M., Gregori, A. (2014): The Freshwater Fish of Slovenia. Zavod Umbra, Ljubljana, 64pp. (in Slovenian)

Povž, M., Leiner, S., Mrakovčić, M., Popović, J. (1990): Rare and endangered fishes from Yugoslavian Adriatic rivers. Journal of Fish Biology, 37, Suppl A, 247-249.

Povž, M., Mrakovčić, M., Kerovec, M. (1997): The first find of Balon's ruffe (Gymnocephalus baloni) in the River Drava in Slovenia and Croatia. Folia Zoologica, 46, 2, 189-190.
Povž, M., Sket, B. (1990): Our Freshwater Fishes. Mladinska knjiga, Ljubljana, 374pp. (in Slovenian)

Povž M, Šumer, S. (2000): Present status and distribution of the species from the genera Misgurnus, Cobitis and Sabanejewia in Slovenia. Folia Zoologica, 49, 1, 107112.

Povž, M., Šumer, S. (2003): Status, distribution and morphometric/meristic characteristics of Cobitis elongata Heckel et Kner 1858 from Slovenia. Folia Biologica (Krakow), 51, Supplement, 21-25.

Povž, M., Šumer, S., Budihna, N. (1998): The Fishes and Crustaceans of the Kolpa River. Založba i2, Ljubljana, 92pp. (in Croatian with English abstract)

Prpa, Z., Treer, T., Piria, M., Šprem, N. (2007): The condition of fish from some freshwaters of Croatia. Ribarstvo, 1, 25-46.

Pustovrh, G., Snoj, A., Sušnik Bajec, S. (2014): Molecular phylogeny of Salmo of the western Balkans, based on multiple nuclear loci. Genetics Selection Evolution, 46, 7, 1-12.

Pušić, I. (1948): Does the trout or marble trout migrate from the Neretva River to the sea?. Ribarstvo Jugoslavije, 3, 7-8, 64. (in Croatian)

Quignard, J. P., Douchement, C. (2003): Alosa fallax nilotica (Geoffroy Saint-Hilaire, 1808). In: Miller, J. P. (ed), The Freshwater Fishes of Europe. Clupeidae, Anguillidae. Vol 2. AULA-Verlag, Wiesbaden, 265-273.

Radaković, M., Delak, M., Žuković, J. (1980): Residues of chlorinated organic insecticides in carp from fish ponds. Hrana i ishrana, 7-8, 187-195. (in Croatian)

Radošević, M. (2013): From gambusia fish to the Paris green chemical: how malaria was overcame in Istria. Journal of Contemporary History, 45, 3, 509-530. (in Croatian with English abstract)

Randić, M. (2011): Kamačnik - a picturesque mountain canyon shaped by the activity of karst waters. Hrvatska vodoprivreda, 194, 99-101. (in Croatian)

Ravnateljstvo Hrvatskog Zemaljskog Zoologičkog Muzeja (1908): List of fish received by the Croatian National Zoological Museum from 1901 to the end of 1905. Glasnik Hrvatskog naravoslovnog društva, 20, 114-126. (in Croatian)

Razpet, A. (2004): Genetic diversity of salmonids of the River Neretva drainage. Master's thesis, Biotechnical Faculty, University of Ljubljana. (in Croatian with English abstract)

Renaud, B. C. (2011): Lampreys of the World. An annotated and illustrated catalogue of lamprey species known to date. FAO Species Catalogue for Fishery Purposes 5, 109pp.

Ribić, D. M. (1909): On trout and fishing for them. Lovačkoribarski viestnik, 18, 42-45. (in Croatian)

Ribić, E. (1901): Fisheries and fish breeding in Bosnia and Herzegovina. Lovačko-ribarski viestnik, 10, 97-98. (in Croatian)

Riđanović, S., Nedić, Z., Riđanović, L. (2014): First 
observation of fish condition from Sava river in Bosnia and Herzegovina. Journal of Survey in Fisheries Sciences, 1, 2, 27-32.

Ristić, M. Đ. (1963): Fisheries and biological study of the Acipenseridae in the Yugoslav section of the lower Danube. Ribarstvo Jugoslavije, 18, 3, 73-80. (in Croatian)

Ristić, M. Đ. (1972a): Biology, age structure, growth and fishing intensity of the bream (Abramis brama L.) in the rivers of the Black Sea basin. Ribarstvo Jugoslavije, 27, 1, 1-13. (in Croatian)

Ristić, M. Đ. (1972b): The catfish (Silurus glanis L.) Biology, distribution and breeding possibilities in the fish ponds of Yugoslavia. Ribarstvo Jugoslavije, 27, 6, 129-139. (in Croatian)

Rödlich, H. F. (1811): Skizzen des physisch-moralischen Zustandes Dalmatiens und der Buchten von Cattaro: Mit einer Charte und neun Kupfertafeln Gebundene Ausgabe. Realschulbuchhandlung, Berlin, 91pp. (in German)

Rössler, E. (1910): Stocking the Sutla with noble fish. Lovačko-Ribarski vjesnik, 19, 59. (in Croatian)

Rössler, E. (1928): Fisheries of Yugoslavia. Ribarski list, 3, 5, 97-103. (in Croatian)

Rössler, E. (1929a): Plitvice Lakes in the fisheries and biological sense. Savić, Sarajevo, 11pp. (in Croatian)

Rössler, E. (1929b): Plitvice Lakes in the fisheries and biological sense. Ribarski list, 4, 5-6, 61-66. (in Croatian)

Rössler, E. (1930): On the fisheries and biological study of waters in the Ogulin area and Gorski Kotar. Ribarski list, 5, 7-8, 88-90. (in Croatian)

Rössler, E. (1931): Fisheries and biological study of the Krka, Cetina and Jadro Rivers. Ribarski list, 6, 7-10, 9498. (in Croatian)

Rössler, E. (1932): Fisheries and biological research of the Lika, Gacka and Dobra Rivers. Ribarski list, 7, 5-6, 83-89. (in Croatian)

Rotarides, M. (1944): Halászat a Dráva-szögletben. Albertina, 1, 185-191. (in Hungarian)

Rüdiger, B., Rüdiger, R. (2002): Biology and egg morphology of the Dalmatian barbelgudgeon Aulopyge huegelii, an endangered species in Croatia. Environmental Biology of Fishes, 63, 4, 451-456.

Rylková, K., Kalous, L., Bohlen, J., Lamatsch, D. K., Petrtýl, M. (2013): Phylogeny and biogeographic history of the cyprinid fish genus Carassius (Teleostei: Cyprinidae) with focus on natural and anthropogenic arrivals in Europe. Aquaculture, 380-383, 13-20.

Ržaničanin, B., Safner, R., Treer, T. (1984a): Results of the first cage breeding of carp (Cyprinus carpio L.) in Vrana Lake near Biograd na moru. Ribarstvo Jugoslavije, 39, 2, 29-31. (in Croatian)

Ržaničanin, B., Treer, T., Safner, R. (1984b): Results of the first cage breeding of catfish (Silurus glanis L.) in Vrana Lake near Biograd na moru. Ribarstvo Jugoslavije, 39, 2, 32-35. (in Croatian)
Ržaničanin, B., Safner, R., Treer, T. (1985): Influence of external factors on the growth of carp (Cyprinus carpio L.) in cage breeding. Ribarstvo Jugoslavije, 40, 2-3, 4951. (in Croatian)

Sabioncello, I. (1953): Pikeperch (Lucioperca lucioperca L.). Ribarstvo Jugoslavije, 8, 3, 56-59. (in Croatian)

Sabioncello, I. (1959): Marked carp caught in the Sava. Ribarstvo Jugoslavije, 14, 3, 75. (in Croatian)

Sabioncello, I. (1962): Contribution to the recognition of grayling in NR Croatia. Ribarstvo Jugoslavije, 17, 1, 9-11. (in Croatian)

Sabioncello, I. (1967): Systematics of freshwater fish. In: Livojević, Z., Bojčić, C. (eds), Priručnik za slatkovodno Ribarstvo. Posebna izdanja "Agronomskog glasnika“, Agronomski fakultet, Zagreb, pp. 21-90. (in Croatian)

Sabioncello, I. (1971a): Tench - Tinca tinca (Linnaeus, 1758). Ribarstvo Jugoslavije, 26, 1, 6-8. (in Croatian)

Sabioncello, I. (1971b): Brown bullhead - Ictalurus nebulosus (Le Sueur, 1816). Ribarstvo Jugoslavije, 26, 3, 52-53. (in Croatian)

Sabioncello, I., Marko, S., Habeković, D. (1964): Fisheries and biological study of Vrana Lake. Ribarstvo Jugoslavije, 19, 4, 82-94. (in Croatian)

Sabioncello, I., Marko, S., Pažur, K. (1969): Fisheries and biological study of the Odra River. Ribarstvo Jugoslavije, 24, 3, 45-47. (in Croatian)

Sabioncello, I., Marko, S., Pažur, K. (1970): Bioecological study of salmonids in SR Croatia. Ribarstvo Jugoslavije, 25, 2, 29-37. (in Croatian)

Safner, R. (1987): Overview of the research on the biology and breeding of the grass carp. Ribarstvo Jugoslavije, 42, 6-9, 6-8. (in Croatian)

Safner, R., Treer, T., Aničić, I., Piria, M., Šprem, N., Matulić, D., Tomljanović, T. (2013): First record of Palmetto bass (Morone saxatilis $\times$ M. chrysops) in the Croatian part of the river Danube. Archives of Biological Sciences 65, 1, 197-200.

Salihćehajić, A. (1965): From the banks of the Sava. Ribarski list, 5, 100-101. (in Croatian)

Sallai, Z., Kontos, T. (2005): Fish faunistical monitoring of the Hungarian part of River Drava (1999-2004). Natura Somogyiensis, 7, 75-104.

Sallai, Z., Kontos, T. (2008): Data to the fish fauna of Croatian Drava sector. In: Purger, J. (ed), Biodiversity studies along the Drava River. University of Pecs, Pecs, pp. 249-273.

Sallai, Z., Mrakovčić, M. (2007): Protocol for sampling and monitoring freshwater ichthyofauna in Drava River. In: Purger, J. (ed), Priručnik za istraživanje bioraznolikosti duž rijeke Drave, Sveučilište u Pečuhu, Pečuh, pp. 133161. (in Croatian)

Salzburger, W., Brandstätter, A., Gilles, A., Parson, W., Hempel, M., Sturmbauer, C., Meyer, A. (2003): Phylogeography of the varione (Leuciscus souffia, Risso 1826) in Central Europe. Molecular Ecology, 12, 23712386. 
Samobor, S. (1935): Fishing in the Bednja Stream. LovačkoRibarski vjesnik, 44, 371-374. (in Croatian)

Schenekar, T., Lerceteau-Köhler, E., Weiss, S. (2014): Finescale phylogeographic contact zone in Austrian brown trout Salmo trutta reveals multiple waves of post-glacial colonization and a pre-dominance of natural versus anthropogenic admixture. Conservation Genetics ,15, 561-572.

Schlosser, J. (1852): Reiseflora aus Süd-Croatien. Oesterreichisches Botanisches Wochenblatt, 2, 49, 385-387. (in German)

Schmall, B., Friedrich, T. (2014a): Die Störarten der Donau Teil 1, Hausen (Huso huso), Europäischer Stör (Acipenser sturio) allochthone Störarten. Österreichs Fischerei, 67, 4, 95-109. (in German)

Schmall, B., Friedrich, T. (2014b): Die Störarten der Donau Teil 2, Waxdick (Acipenser gueldenstaedtii), Glattdick (Acipenser nudiventris), Sternhausen (Acipenser stellatus) und historische Störnachweise zweifelhafter Identität. Österreichs Fischerei, 67, 5-6, 129-143. (in German)

Schmall, B., Friedrich, T. (2014c): Die Störarten der Donau Teil 3, Sterlet, „Stierl“ (Acipenser ruthenus) und aktuelle Schutzprojekte im Donauraum. Österreichs Fischerei, 67,7, 167-183. (in German)

Schmall, B., Friedrich, T. (2014d): Das Schicksal der großen Störarten in der Oberen Donau. Denisia, 33, 432-442. (in German)

Schneider, D. (1998): Taxonomical and Biological Characteristics of Delta Neretva Loaches Cobitis taenia (Pisces, Cobitidae). Master's thesis, Faculty of Science, University of Zagreb. (in Croatian with English abstract)

Schneider, D., Mrakovčić, M., Mustafić, P., Kerovec, M. (2000b): Morphological differences in some Cobitis populations from Croatia. Folia Zoologica, 49, 1, 227234.

Schneider, D., Mustafić, P., Mrakovčić, M., Mihaljević, Z. (2000a): Some aspects of the biology of the Neretvan spined loach. Folia Zoologica, 49, 1, 159-165.

Schöffmann, J. (1991): Die Weichmaulforelle (Salmothymus obtusirostris, Heckei 1851), eine bedrohte Salmonidenart in Dalmatien. Österreichs Fischerei, 44, 64-66. (in German)

Schöffmann, J. (2003): Zur aktuellen Situation der vier Unterarten der Weichmaulforelle, Salmo (Salmothymus) obtusirostris Heckel, 1851. Österreichs Fischerei, 56, 180-184. (in German)

Schöffmann, J. (2004): Nachweis einer Restpopulation der bereits für ausgestorben erklärten Weichmaulforelle, Salmo (Salmothymus) obtusirostris ssp., der Vrlika, südliches Kroatien. Österreichs Fischerei, 57, 277-278. (in German)

Schulz, N. (1985): Das Wachstum des Huchens (Hucho hucho L.) in der Drau in Kärnten. Österreichs Fischerei, 38, 133-142. (in German)

Sebišanović, Gj. (1880): Contributions to the fauna surrounding Karlovac. Jahresbericht der k. k. OberRealschule in Rakovac im kroatische Grenzlande fur das Schuljahr 1880/81, 3-24. (in Croatian)

Sebišanović, Gj. (1881): On the fish in the Mrežnica near Mostanje. Jahresbericht der k. k. Ober-Realschule in Rakovac im kroatische Grenzlande fur das Schuljahr 1880/81, 29-31. (in Croatian)

Sebišanović, Gj. (1888): Gobio uranoscopus Ag. Glasnik Hrvatskog naravoslovnog društva, 3, 271. (in Croatian)

Sebišanović, Gj. (1889a): A contribution to the nomenclature of our fish. Glasnik Hrvatskog naravoslovnog društva, 1-5, 186-194. (in Croatian)

Sebišanović, Gj. (1889b): Zoophenological news. Glasnik Hrvatskog naravoslovnog društva, 1-5, 233-234. (in Croatian)

Sebišanović, Gj. (1889c): This year's spawning of the nase in the Drava near Varaždin. Glasnik Hrvatskog naravoslovnog društva, 1-5, 234. (in Croatian)

Sebišanović, Gj. (1890): Ichthyological reflections. Tiskarski zavod Platzera sina, Varaždin, 82pp. (in Croatian)

Seeley, H. G. (1886): The Fresh-water fishes of Europe. Cassell \& Company, London, 444pp.

Seljan, D. (1843): Map of the Illyrian provinces: or a mirror of a land inhabited by the Illyrian-Slavic people with a description of the hills, streams, cities and numerous places near the present class, with a short enclosure and attached landscape or map. Part I (Austrian-llyric provinces): Kr. p. ilirska tiskarna Dra. Ljudevita Gaja, Zagreb, 256pp. (in Croatian)

Sella, M. (1927): I pesci larvifagi e l'esperimento di campagna antimalarica con le Gambusie a Rovigno d'Istria. Rivista di Malariologia, 6, 6, 881-909. (in Italian)

Sella, M. (1928): I pesci larvifagi e l'esperimento di campagna antimalarica con le Gambusie a Rovigno d'Istria. Bollettino di pesca, piscicoltura e idrobiologia, 4, 2, 174-197. (in Italian)

Sella, M. (1929): Gambusia e Verde di Parigi nella lotta antimalarica a Rovigno e cenni sulla lotta in Istria. Rivista di Malariologia, 8, 4, 367-392. (in Italian)

Sevcsik, A., Erös, T. (2008): A revised catalogue of freshwater fishes of Hungary and the neighbouring countries in the Hungarian Natural History Museum (Pisces). Annales Historico-Naturales Musei Nationalis Hungarici, 100, 331-383.

Shubina, T. N., Popova, A. A., Vasil'ev, V. P. (1989): Acipenser stellatus Pallas, 1771. In: Holčík, J. (ed), The Freshwater Fishes of Europe. Vol 1, Part II, General introduction to fishes, Acipenseriformes. AULA-Verlag, Wiesbaden, pp. 395-443.

Siebold, C. T. E. (1863): Die Süsswasserfische von Mitteleuropa. Engelmann, Leipzig, 431pp. (in German)

Simonović, P. (2001): The fishes of Serbia. NNK Internacional, Beograd, Biološki fakultet Univerziteta u Beogradu, Beograd, Zavod za zaštitu prirode Srbije, Novi Beograd, 247pp. (in Serbian)

Simonović, P., Budakov, Lj., Nikolić, V., Marić, S. (2005): 
Recent record of the ship sturgeon Acipenser nudiventris in the middle Danube (Serbia). Biologia Bratislava, 60, 2, 1-2.

Simonović, P., Piria, M., Zuliani, T., Ilić, M., Marinković, N., Kračun-Kolarević, M., Paunović, M. (2017b): Characterization of sections of the Sava River based on fish community structure. Science of the Total Environment, 574, 264-271.

Simonović, P., Tošić, A., Škraba Jurlina, D., Nikolić, V., Piria, M., Tomljanović, T., Šprem, N., Mrdak, D., Milošević, D., Bećiraj, A., Dekić, R., Povž, M. (2017a): Diversity of Brown trout Salmo cf. trutta in the River Danube Basin of Western Balkans as assessed from the structure of their mitochondrial control region haplotypes. Journal of Ichthyology, 57, 4, 603-616.

Skalin, B. (1990): Huchen as an endangered fish species. Ribarstvo Jugoslavije, 45, 1-2, 22-30. (in Croatian)

Skaramuca, B., Teskeredžić, Z., Teskeredžić, E. (1997): Mariculture in Croatia, history and perspectives. Ribarstvo, 55, 1, 19-25.

Smlatić, S. (1961): Una - a unique river. Priroda, 48, 8, 225-229. (in Croatian)

Snoj, A., Bogut, I., Sušnik, S. (2008): Evidence of a genetically distinct population of Vrljika softmouth trout Salmo obtusirostris Heckel evolved by vicariance. Journal of Fish Biology, 72, 1945-1959.

Snoj, A., Melkič, E., Sušnik, S., Muhamedagić, S., Dovč, P. (2002): DNA phylogeny supports revised classification of Salmothymus obtusirostris. Biological Journal of the Linnean Society, 77, 399-411.

Snoj, A., Razpet, A., Tomljanović, T., Treer, T., Sušnik, S. (2007): Genetic composition of the Jadro softmouth trout following translocation into the new habitat. Conservation Genetics, 8, 1213-1217.

Sofradžija, A. (2009): The freshwater fish of Bosnia and Herzegovina. Vijeće Kongresa bošnjačkih intelektualaca, Sarajevo, 355pp. (in Bosnian)

Sofradžija, A., Berberović, Lj. (1972): Comparative karioloic research of the species Paraphoxinus alepitodus, $P$. adspersus, P. pstrossi, P. metohiensis and P. croaticus. Godišnjak biološkog instituta Univerziteta u Sarajevu, 25, 135-173. (in Bosnian)

Sofradžija, A., Hadžiselimović, R. (1981): Chromosomes of the freshwater lampreys and fish of Yugoslavia. Godišnjak biološkog instituta Univerziteta u Sarajevu, 34, 117-152. (in Bosnian)

Sokal, D. (1961): Disorderly state of fisheries on the Sava. Ribarski list, 2-3, 41. (in Croatian)

Sokolov, L. I., Vasil'ev, V. P. (1989a): Acipenser ruthenus Linnaeus, 1758. In: Holčík, J. (ed), The Freshwater Fishes of Europe. Vol 1, Part II, General introduction to fishes, Acipenseriformes. AULA-Verlag, Wiesbaden, pp. 227-262.

Sokolov LI, Vasil'ev, V. P. (1989b): Acipenser nudiventris Lovetsky, 1828. In: Holčík, J. (ed), The Freshwater Fishes of Europe. Vol 1, Part II, General introduction to fishes, Acipenseriformes. AULA-Verlag, Wiesbaden, pp. 206-226.

Stanković, D., Crivelli, A. J, Snoj, A. (2015): Rainbow trout in Europe: introduction, naturalization, and impacts. Reviews in Fisheries Science \& Aquaculture, 23 (1): 3971.

Steindachner, F. (1866a): Über eine neue Telestes-Art aus Croatien. Sitzungsberichte der Kaiserlichen Akademie der Wissenschaften in Wien, 54, 1, 300-302. (in German)

Steindachner, F. (1866b): Ichthyologische Notizen (II). Zur Flussfischfauna von Croatien. Sitzungsberichte der Kaiserlichen Akademie der Wissenschaften, Mathematisch-Naturwissenschaftliche Classe, 52, 1, 594-599. (in German)

Steindachner, F. (1874): Beschreibung einer neuen Art und Gattung aus der Familie der Pleuronectiden und einer neuen Thymallus-Art. Anzeiger der Kaiserlichen Akademie der Wissenschaften, MathematischNaturwissenschaftliche Classe, Wien 11, 171-172. (in German)

Steindachner, F. (1875): Über eine neue Gattung und Art aus der Familie der Pleuronectiden und über eine neue Thymallus-Art. Sitzungsberichte der Kaiserlichen Akademie der Wissenschaften. MathematischNaturwissenschaftliche Classe, 70, 363-371. (in German)

Steindachner, F. (1882): Beiträge zur Kenntniss der Fische Afrika's (II): und Beschreibung einer neue ParaphoxinusArt aus der Herzegowina. Sitzungsberichte der Kaiserlichen Akademie der Wissenschaften in Wien, 45, 1-28. (in German)

Steindachner, F. (1882): Ichthyologische Beitrage (XII). Sitzungsberichte der Kaiserlichen Akademie der Wissenschaften in Wien, 1, 86, 61-82. (in German)

Steindachner, F. (1895): Beiträge zur Kenntniss der Süsswasserfische der Balkan-Halbinsel. Denkschriften der Akademie der Wissenschaften. MathematischNaturwissenschaftliche Classe, 63, 181-188. (in German)

Steindachner, F. (1901): Bericht über ein Vorkommen einer bisher noch unbeschriebenen Paraphoxinus-Art. Anzeiger der Kaiserlichen Akademie der Wissenschaften. Mathematisch Naturwissenschaftliche, 38, 18, 197198. (in German)

Stepanek, O. (1956): Stocking the reservoirs. Ribarski list, 5,111 . (in Croatian)

Stepanek, O. (1965): Catfish. Ribarski list, 5, 112-115. (in Croatian)

Stepanek, O. (1966): Diet of fish and sports fishing. Ribarski list, 2, 53. (in Croatian)

Steuer, A. (1899): Die Entomostraken der Plitvicer Seen und des Blata Sees (Kroatien), gesamelt von $\mathrm{Dr} \mathrm{R}$. Sturnay 1885. Annalen des Naturhistorischen Museums in Wien, 13, 159-188. (in German)

Stevanović, D. (1960): Our trout. Ribarski list, 4, 85-86. (in 
Bosnian)

Stevanović, D. (1961): Killers on saturday. Ribarstvo Jugoslavije, 16, 2, 55-56. (in Bosnian)

Stevanović, D. (1969a): The secret life of the beluga sturgeon. Ribarski list, 4, 104. (in Bosnian)

Stevanović, D. (1969b): Eel races. Ribarski list, 6, 132. (in Bosnian)

Stierandová, S., Vukić, J., Vasil'eva, E. D., Zogaris, S., Shumka, S., Halačka, K., Vetešník, L., Švátora, M., Nowak, M., Stefanov, T., Koščo, J., Mendel, J. (2016): A multilocus assessment of nuclear and mitochondrial sequence data elucidates phylogenetic relationships among European spirlins (Alburnoides, Cyprinidae). Molecular Phylogenetics and Evolution, 94, 479-491.

Stojnić, E. (1965): Marine trout on the agenda again. Morsko Ribarstvo, 17, 9-10, 177. (in Croatian)

Suić, J. (2005): Model of fisheries management of salmonid waters of the Gacka River. Master's thesis, Faculty of Science, University of Zagreb. (in Croatian with English abstract)

Suić, J., Šarić, M., Homen, Z., Jahutka, I., Mišura, A. (2008): Commercial fishing in the freshwaters of the Republic of Croatia in 2006. Ribarstvo, 1, 17-24. (in Croatian with English abstract)

Sušnik, S., Weiss, S., Odak, T., Delling, B., Treer, T., Snoj, A. (2007): Reticulate evolution: ancient introgression of the Adriatic brown trout mtDNA in softmouth trout Salmo obtusirostris (Teleostei: Salmonidae). Biological Journal of the Linnean Society, 90, 139-152.

Szczerbowski, J. A. (2002): Carassius auratus (Linnaeus, 1758). In: Bănărescu, P. M., Paepke, H-J. (eds), The Freshwater Fishes of Europe. Cyprinidae 2/III. Vol 5/III. AULA-Verlag, Wiebelsheim, pp. 5-41.

Szczerbowski, J. A., Szczerbowski, A. J. (2002): Carassius carassius. In: Bănărescu, P. M., Paepke, H-J. (eds), The Freshwater Fishes of Europe. Cyprinidae 2/III. Vol 5/III. AULA-Verlag, Wiebelsheim, pp. 43-78.

Šafarek, G. (2010): Kamačnik - protected landscape, canyon beauty. Euro city, putna revija Hž-putničkog prijevoza, 18, 67, 88-89. (in Croatian)

Šanda, R., Balković, I., Bogut, I., Galović, D., Vidaković, J., Čerba, D., Kovačević, T. (2013): New data on the invasive gobiid species distribution. Knjiga sažetaka. 9. međunarodnog gospodarsko-znanstvenog skupa o ribarstvu "Hrvatska akvakultura u Europskoj uniji sadašnjost i budućnost". 9. Međunarodni gospodarskoznanstveni skup o ribarstvu "Hrvatska akvakultura u Europskoj uniji - sadašnjost i budućnost", Vukovar (Hrvatska), svibanj 2013. Hrvatska gospodarska komora.

Šanda, R., Bogut, I., Doadrio, I., Kohout, J., Perdices, A., Perea, S., Šediva, A., Vukić, J. (2008): Distribution and taxonomic relationships of spined loaches (Cobitidae, Cobitis): in the River Neretva basin, Bosnia and Herzegovina. Folia Zoologica, 57, 1-2, 20-25.

Šanda, R., Kovačić, M. (2009): Freshwater gobies in the
Adriatic drainage basin of the Western Balkans. Annales Series Historia Naturalis, 9, 1, 1-10.

Šedivá, A., Janko, K., Šlechtová, V., Kotlík, P., Simonović, P., Delić, A., Vassilev, M. (2008): Around or across the Carpathians: colonization model of the Danube basin inferred from genetic diversfications of stone loach (Barbatula barbatula) populations. Molecular Ecology, 17, 5, 1277-1292.

Šiljeg, M. (2012): Ecological state of the Neretva River. Hrvatski neretvanski zbornik, 4, 84-96. (in Croatian)

Šimunčić, A. (1902): A strange occurrence. Lovačkoribarski viestnik, 11, 95. (in Croatian)

Škovrlj, N. (1953a): The Krka near Knin. Ribarstvo Jugoslavije, 8, 5, 106-108. (in Croatian)

Škovrlj, N. (1953b): Protecting the softmouth trout. Ribarstvo Jugoslavije, 8, 4, 88-89. (in Croatian)

Škraba, D., Bećiraj, A., Šarić, I., Ićanović, I., Džaferović, A., Piria, M., Dekić, R., Tošić, A., Nikolić, V., Simonović, P. (2017): Haplotype diversity of brown trout (Salmo trutta L.) populations from Una River drainage area in Bosnia and Herzegovina: implications for conservation and fishery management. Acta Zoologica Bulgarica 69, 1, 25-30.

Šlechtová, V., Bohlen, J., Freyhof, J., Persat, H., Delmastro, G. B. (2004): The Alps as barrier to dispersal in coldadapted freshwater fishes? Phylogeographic history and taxonomic status of the bullhead in the Adriatic freshwater drainage. Molecular Phylogenetics and Evolution, 33, 225-239.

Šloser, J. K. K. (1870): Kalnička gora and its natural features. Rad Jugoslavenske akademije znanosti i umjetnosti, 11, 145-227. (in Croatian)

Šoljan, T. (1931): A special catch of eel from Vrana Lake. Ribarski list, 6, 1-4, 16-18. (in Croatian)

Šoljan, T. (1948): Fauna and flora of the Adriatic: Fish I. Institut za Oceanografiju i Ribarstvo, FNR Jugoslavije, Split, 437pp. (in Croatian)

Šoljan, T. (1975): I pesci dell Adriatico, per pescatori, specialisti e appassionati. Arnoldo Mondadori Editore, Verona, 523pp. (in Italian)

Šorić, V. M. (2007): Taxonomic status of the species of the genus Leuciscus (Pisces, Cyprinidae) from the Adriatic and Aegean basins. Kragujevac Journal of Science, 29, 155-169.

Šorić, V. M., Bănărescu, P. M. (1999): Aulopyge huegeli Heckel, 1841. In: Bănărescu, P. M. (ed), The Freshwater Fishes of Europe. Cyprinidae 2/I. Vol. 5/I. AULA-Verlag, Wiebelsheim, pp. 369-381.

Šoštarić, D. (1888): Contribution to the understanding of the freshwater vertebrates of Croatia. Rad Jugoslavenske akademije znanosti i umjetnosti, 22, 103-214. (in Croatian)

Šprem, N., Matulić, D., Treer, T., Aničić, I. (2010): A new maximum length and weight for Scardinius erythrophthalmus. Journal of Applied Ichthyology, 26, 4, 618-619. 
Šprem, N., Piria, M., Treer, T. (2001): Morphologic parameters and length-mass relationship of three roach (Rutilus rutilus (L., 1758.)) populations from northwestern Croatia. Ribarstvo, 3, 99-106. (in Croatian)

Šprem, N., Tomljanović, T., Piria, M., Treer, T., Safner, R., Aničić, I. (2005a): Condition and CPUE of European Grayling (Thymallus thymallus L.) population in the Croatian Kupa river. Journal of Central European Agriculture, 6, 4, 569-576.

Šprem, N., Matulić, D., Treer, T., Aničić, I. (2005c): A new maximum length and weight for Scardinius erythrophthalmus. Journal of Applied Ichthyology, 26, 618-619.

Šprem, N., Tomljanović, T., Piria, M., Treer, T., Safner, R., Aničić, I. (2005b): The growth of grayling (Thymallus thymallus L.) in the Kupa River. Ribarstvo, 2, 39-46. (in Croatian with English abstract)

Štefanac, M. (1973): Grayling in Lika waters. Ribarstvo Jugoslavije, 28, 2, 45. (in Croatian)

Štefanac, M. (1974): The growth rate of brown trout and rainbow trout in the Gacka River and its tributaries, in relation to the global standards of Dr. Einselle. Ribarstvo Jugoslavije, 29, 2, 39-41. (in Croatian)

Štefanac, M. (1986): Some observations on the population of brown trout (Salmo trutta m. fario L.) in the Gacka River. Ribarstvo Jugoslavije, 41, 4-5, 102. (in Croatian)

Štefanac, M., Bunjevčević, Z. (1982): A contribution to the understanding of the morphological characteristics of brown trout of the Gacka River. Ribarstvo Jugoslavije, 37, 2, 30-32. (in Croatian)

Tadić, A. (1931): Stellate sturgeon. Ribarski list, 6, 7-10, 114. (in Serbian)

Tadić, A. (1932): Zoological rarity in the Podunavlje region. Priroda, 22, 9-10, 288-289. (in Serbian)

Tadić, A. (1933): Exhibit of freshwater and marine fishes in Novi Sad. Ribarski list, 8, 3-4, 44-45. (in Serbian)

Tadić, A. (1959): Lampreys (cyclostomata). Morsko Ribarstvo, 11, 6, 110-112. (in Serbian)

Tadić, A. (1966): The fish world and the Djerdjap hydropower plant. Priroda, 53, 9, 273. (in Serbian)

Taler, Z. (1926): From our waters. Športski ribar, 1, 1, 1719. (in Croatian)

Taler, Z. (1931): A 16 kg trout. Ribarski list, 6, 1-4, 22-23. (in Croatian)

Taler, Z. (1935a): Premature rainbow trout. Ribarski vjesnik, 13, 12, 300. (in Croatian)

Taler, Z. (1935b): On the upper course of the Kupa River. Ribarski vjesnik, 13, 12, 285-293.

Taler, Z. (1938): Rainbow trout in the Gacka River. Ribarstvo, 3-6, 84-87. (in Croatian)

Taler, Z. (1944): Grayling (Thymallus thymallus L.), its distribution in Croatia and in the Balkans, and its economic value. Zagreb, 87pp. (in Croatian)

Taler, Z. (1945a): Nase - Chondrostoma nasus (Linne).
Zoogeographic data, dietary and commercial value, and various observations. Nakladni zavod Hrvatske, Zagreb, 88pp. (in Croatian)

Taler, Z. (1945B): Huchen (Salmo hucho L.) and Neretva marble trout (Salmo marmoratus Cuv.) zoogeographic dana and commercial value. Pučka knjižara Priroda, Zagreb, pp. 71-77. (in Croatian)

Taler, Z. (1946a): A $70 \mathrm{~kg}$ beluga sturgeon. Ribarstvo Jugoslavije, 1, 1, 10. (in Croatian)

Taler, Z. (1946b): Transfer of rainbow trout from Slovenia to Slavonia. Ribarstvo Jugoslavije, 1, 1, 17. (in Croatian)

Taler, Z. (1946c): Transfer of the roe of rainbow and domestic trout from Slovenia to Samobor. Ribarstvo Jugoslavije, 1, 2, 22. (in Croatian)

Taler, Z. (1947): About an evil act in fisheries. Ribarstvo Jugoslavije, 2, 5, 92. (in Croatian)

Taler, Z. (1948a): Discovery on the Gacka and its significance for the breeding of trout. Ribarstvo Jugoslavije, 3, 1-2, 8-11. (in Croatian)

Taler, Z. (1948b): Sterlet, sturgeons and their relatives. Ribarstvo Jugoslavije, 3, 7-8, 57. (in Croatian)

Taler, Z. (1948c): Appearance of eels in the Gacka River and possibility of increasing its catch. Ribarstvo Jugoslavije, 3, 9-10, 96-97. (in Croatian)

Taler, Z. (1948d): Stocking the trout streams around Zagreb. Ribarstvo Jugoslavije, 3, 9-10, 103. (in Croatian)

Taler, Z. (1948e): The case of swim bladder disease in trout. Ribarstvo Jugoslavije, 3, 5, 38-41. (in Croatian)

Taler, Z. (1948f): Catfish and its significance in fisheries. Ribarstvo Jugoslavije, 3, 9-10, 81-82. (in Croatian)

Taler, Z. (1949a): Dalmatian barbelgudgeon. Ribarstvo Jugoslavije, 4, 10-12, 114-115. (in Croatian)

Taler, Z. (1949b): Yield of marked trout int he Gacka River and Ohrid Lake. Ribarstvo Jugoslavije, 4, 5, 48-49. (in Croatian)

Taler, Z. (1950a): Visovac lake trout (Salmo visovacensis n. sp.). Glasnik Hrvatskoga Prirodoslovnoga društva, 3, 118-158. (in Croatian)

Taler, Z. (1950b): Exceptional significance of the Gacka fish farm. Slatkovodno Ribarstvo Jugoslavije, 5, 5, 74-76. (in Croatian)

Taler, Z. (1950c): Which salmonids live in Yugoslavia and which are farmed. Slatkovodno Ribarstvo Jugoslavije, 5, 3-4, 58-60. (in Croatian)

Taler, Z. (1951a): Softmouth trout. Slatkovodno Ribarstvo Jugoslavije, 6, 3, 62-64. (in Croatian)

Taler, Z. (1951b): Protection of our rare and unusual fish. Slatkovodno Ribarstvo Jugoslavije, 6, 10, 224-227. (in Croatian)

Taler, Z. (1951c): Mutual relations between native brown trout and newly translocated trout from stocking. Slatkovodno Ribarstvo Jugoslavije, 6, 12, 285-287. (in Croatian)

Taler, Z. (1951d): Third report on marking trout on the Gacka River. Slatkovodno Ribarstvo Jugoslavije, 6, 3, 
60-62. (in Croatian)

Taler, Z. (1951e): On fish stocking. Slatkovodno Ribarstvo Jugoslavije, 6, 5, 127-130. (in Croatian)

Taler, Z. (1951f): Sturgeons - Acipenseridae. Slatkovodno Ribarstvo Jugoslavije, 6, 5, 183-185. (in Croatian)

Taler, Z. (1951g): Underground fish in our karst. Slatkovodno Ribarstvo Jugoslavije, 6, 4, 107-109. (in Croatian)

Taler, Z. (1952a): The trout of the Plitvice Lakes and their protection. Ribarstvo Jugoslavije, 7, 4, 25-27. (in Croatian)

Taler, Z. (1952b): Protection of the Krka Softmouth trout a natural rarity in the fish world. Ribarstvo Jugoslavije, $7,7,122$. (in Croatian)

Taler, Z. (1952c): The problem of the Buna River. Ribarstvo Jugoslavije, 7, 11-12, 186-188. (in Croatian)

Taler, Z. (1952d): Experiences in breeding salmonids in Yugoslavia and guidelines for stocking open waters, and the farming of fish for consumption. Ribarstvo Jugoslavije, 7, 1-2, 12-15. (in Croatian)

Taler, Z. (1953a): Distribution and list of the freshwater fishes of Yugoslavia. Glasnik Prirodnjačkog muzeja Srpske zemlje B 5-6, 425-455. (in Croatian)

Taler, Z. (1953b): Good success in the domestication of trout in the Korenička Matica. Ribarstvo Jugoslavije, 8, 2 , 48. (in Croatian)

Taler, Z. (1953c): Pike (Esox lucius Linne). Ribarstvo Jugoslavije, 8, 4, 71-75. (in Croatian)

Taler, Z. (1953d): Two cases of the translocation of carp and tench into other waters. Ribarstvo Jugoslavije, 8, 5, 110-112. (in Croatian)

Taler, Z. (1953e): The Sturba and Šuica Rivers in western Bosnia, compared with the Gaska River in Lika. Ribarski list, 1, 4-6. (in Croatian)

Taler, Z. (1953f): Noble and distinctive fish in Bosnia and Herzegovina. Ribarski list, 4, 56-57. (in Croatian)

Taler, Z. (1953g): On the significance of the huchen and its breeding. Ribarski list, 5, 83-85. (in Croatian)

Taler, Z. (1954a): Softmouth trout. Ribarski list, 1, 2-4. (in Croatian)

Taler, Z. (1954b): The Krka and the issue of its protection. Ribarstvo Jugoslavije, 9, 1, 23-24. (in Croatian)

Taler, Z. (1954c): The need to align the interests of hydropower with the interests of fisheries on the Krka River in Dalmatia. Ribarstvo Jugoslavije, 9, 2, 42-44. (in Croatian)

Taler, Z. (1954d): Rainbow trout and its domestication in Yugoslavia as a fisheries, biological and commercial issue. Ribarstvo Jugoslavije, 9, 4, 82-89. (in Croatian)

Taler, Z. (1958): A contribution to the study of the Plitvice trout. In: Šafar, J. (ed), Plitvička jezera Nacionalni park. Nacionalni park Plitvička jezera, Zagreb, 329-353. (in Croatian)

Talpaš (1901): Fish of trouts. Lovačko-ribarski viestnik, 10, 102-105. (in Croatian)
Taube, F. W. (1777): Historische und geographische Beschreibung des Königreiches Slavonien und des Herzogthumes Syrmien: sowol nach ihrer natürlichen Beschaffenheit, als auch nach ihrer itzigen Verfassung und neuen Einrichtung in kirchlichen, bürgerlichen und militarischen Dingen. Leipzig, 331 pp. (in German)

Telar, S. (1925): The release of trout fry into streams. Ribarski vjesnik, 3, 10-11, 57-64. (in Croatian)

Teskeredžić, E. (1983): Breeding rainbow trout (Salmo gairdneri Rich in Žrnovnica Bay near Jurjevo. Morsko Ribarstvo, 35, 1, 16-17. (in Croatian)

Teskeredžić, E. (1984): The influence of brackish water on the quality of meat of rainbow trout (Salmo gairdneri). $\mathrm{PhD}$ thesis, Faculty of Veterinary Medicine, University of Zagreb. (in Croatian)

Teskeredžić, E., Edwards, D. J. (1987): Coho thrive in Adriatic trials. Fish Farmer, 2, 36-37.

Teskeredžić, E., Teskeredžić, Z. (1990): A successful rearing experiment with amago salmon (Oncorhynchus masou rhodurus): in floating cages in the Adriatic Sea. Aquaculture, 86, 201-208.

Teskeredžić, E., Teskeredžić, Z., Malnar Z, Hacmanjek, M., Marguš, D. (1986): The effect of stocking density on growth and mortality of rainbow trout cultured in floating cages in the brackish water of the River Krka estuary. Ichthyologia, 18, 1, 41-46.

Teskeredžić, E., Teskeredžić, Z., McLean, E., Tomec, M., Čož-Rakovac, R. (1993): Occurrence, distribution, and potential future of Yugoslavian salmonids. In: Cloud, G. J., Thorgaard, H. G. (eds), Genetic Conservation of Salmonid Fishes. NATO ASI Series, Series A: Life Sciences, Plenum Press, New York, 243-252.

Teskeredžić, E., Teskeredžić, Z., Tomec, M., Hacmanjek, M. (1989): Culture of coho salmon (Oncorhynchus kisutch): and rainbow trout (Salmo gairdneri) in the Adriatic Sea. World Aquaculture, 20, 56-67.

Teskeredžić, Z., Pfeifer, K. (1986): The meat quality of rainbow trout, Salmo gairdneri cultured in the brackish water. Ichthyologia, 18, 1, 15-22.

Thacker, C. E., Gkenas, C., Triantafyllidis, A., Malavasi, S., Leonardos, I. (2019): Phylogeny, systematics and biogeography of the European sand gobies (Gobiiformes: Gobionellidae). Zoological Journal of the Linnean Society, 185, 1, 212-225.

Thaller, Z. (1929): The fateful reform of fishing maps in BiH. Ribarski list, 4, 1-2, 7. (in Croatian)

Thaller, Z. (1932): Freshwater fish. In: Thaller, Z. (ed), Vode i ribe Jugoslavije u slici i riječi. Tipografija, Zagreb, 5965. (in Croatian)

Thaller, Z. (1936): Marble trout and huchen. Priroda, 26, 8, 230-234. (in Croatian)

Tomljanović, T. (2014): Endemic softmouth trout Solinka (Salmo obtusirostris salonitana). Tusculum, 7, 215-224. (in Croatian with English abstract)

Tomljanović, T., Fabijanić, N., Treer, T., Matulić, D., Piria, M., Šprem, N., Aničić, I., Safner, R. (2012b): 
Influence of invasive fish species Prussian carp (Carassius gibelio) on Croatian ichthyocenose. In: Pospišil, M., (ed), Proceedings of $47^{\text {th }}$ Croatian \& $7^{\text {th }}$ International Symposium of Agriculture. 47th Croatian $\& 7^{\text {th }}$ International Symposium of Agriculture, Opatija (Croatia), February 2012. Faculty of Agriculture, University of Zagreb, Zagreb, pp. 634-636. (in Croatian)

Tomljanović, T., Piria, M., Šprem, N., Matulić, D., Zanella, D. (2013b): Finding of Sterlet (Acipenser ruthenus) in the Sava river near Zagreb. Conference Proceedings. VI International Conference "Water \& Fish", BelgradeZemun, Serbia: 326-330.

Tomljanović, T., Piria, M., Treer, T., Safner, R., Šprem, N., Aničić, I., Matulić, D., Kordić, V. (2011): Morphological parameters of common carp (Cyprinus carpio carpio L.) populations in Republic of Croatia. Ribarstvo, 69, 3, 8193. (in Croatian with English abstract)

Tomljanović, T., Treer, T., Aničić, I., Safner, R., Šprem, N. (2012a): Threatened fishes of the world: Salmo obtusirostris salonitana (Karaman, 1926) (Salmonidae). Ribarstvo, 70, 2, 61-64.

Tomljanović, T., Treer, T., Ćubrić, V. Č., Safner, R., Šprem, N., Piria, M., Matulić, D., Safner, R., Aničić, I. (2013a): Microsatellite-based genetic variability and differentiation of hatchery and feral common carp Cyprinus carpio L. (Cyprinidae; Cypriniformes) populations in Croatia. Archives of Biological Sciences, $65,2,577-584$.

Tomljanović, T., Treer, T., Piria, M., Aničić, I., Safner, R., Šprem, N. (2007): Molecular trait of soft-muzzled trout (Salmothymus obtusirostris salonitana) and its maintaining. In: Pospišil, M. (ed), Book of abstracts of the $42^{\text {th }}$ Croatian and $2^{\text {nd }}$ International Symposium of Agriculture. $42^{\text {th }}$ Croatian and $2^{\text {nd }}$ International Symposium of Agriculture, Opatija (Croatia), February 2007, Faculty of Agriculture, University of Zagreb, Zagreb, 180-181.

Topić Popović, N., Strunjak-Perović, I., Čož-Rakovac, R. (2000): Aeromonas hydrophila isolated from wild freshwater fish in Croatia. Veterinary Research Communications, 24, 6, 371-377.

Topić Popović, N., Hacmanjek, M., Teskeredžić, E. (2001): Health status of rudd (Scardinius erythrophthalmus hesperidicus H.): in Lake Vrana on the Island of Cres, Croatia. Journal of Applied Ichthyology, 17 (1): 43-45.

Tortonese, E. (1989): Acipenser naccarii Bonaparte, 1836. In: Holčík, J. (ed), The Freshwater Fishes of Europe. Vol 1, Part II, General introduction to fishes, Acipenseriformes. AULA-Verlag, Wiesbaden, pp. 285293.

Toth, J., Biro, P. (1982): Exotic fish species acclimatized in Hungarian natural waters. V.2 - Introductions and Transplantations. FAO, EIFAC Technical Paper 42 (1-2): 550-554.

Tóth, J., Mikuska, J. (1971): Similar changes in the ichthyofauna in the Yugoslav and Hungarian sections of the Danube. Ribarstvo Jugoslavije, 26, 6, 119-124. (in Croatian)

Treer, T. (1985): The influence of cage rearing of fish on the development dynamics of zooplanktonic organisms. Poljoprivredna znanstvena smotra 68, 97-112. (in Croatian)

Treer, T. (1987): The influence of bentic on pelagic zone in cage fish culture. Ichthyologia, 19, 1, 69-75.

Treer, T. (1989): An overview of the research of Vrana Lake near Biograd na moru. Morsko Ribarstvo, 41, 4, 135138. (in Croatian)

Treer, T., Aničić, I., Safner, R. (1991): Some water chemical variables in correlation with fish cage culture mass in shallow lake. Ichthyologia, 23, 1, 91-98.

Treer, T., Aničić, I., Safner, R. (1995): The growth and condition of common carps (Cyprinus carpio) introduced into Croatian Vransko lake. Ribarstvo, 53, 2, 63-73.

Treer, T., Aničić, I., Safner, R., Odak, T., Piria, M. (2003): Note on the growth of endemic soft-muzzled trout Salmothymus obtusirostris translocated into a Dalmatian river. Biologia, 58, 5, 999-1001.

Treer, T., Aničić, I., Safner, R., Odak, T., Piria, M. (2008): Growth and condition of endemic trout Salmothymus obtusirostris in a Dalmatian river Jadro. In: Nielsen, J. L., Dodson, J. J., Friedland, K., Hamon, T. R., Musick, J., Verspor, E. (eds), Reconciling fisheries with conservation. American fisheries society, Maryland, pp. 1771-1776.

Treer, T., Aničić, I., Safner, R., Odak, T., Piria, M. (2005): Post-spawning condition of endemic soft-muzzled trout Salmothymus obtusirostris in the Žrnovnica river. Ribarstvo, 63, 3, 85-90.

Treer, T., Aničić, I., Safner, R., Opačak, A. (1989): Influence of cage rearing of fish on water chemistry. Ribarstvo Jugoslavije, 44, 6, 135-138. (in Croatian)

Treer, T., Aničić, I., Safner, R., Odak, T., Piria, M. (2004): Growth and condition of endemic trout Salmothymus obtusirostris in a Dalmatian river Jadro. Fourth World Fisheries Congress. 285pp.

Treer, T., Habeković, D., Aničić, I., Safner, R., Kolak, A. (1997): Standard growth curve for chub (Leuciscus cephalus L. 1758) in Croatia. Ribarstvo, 55, 2, 47-52.

Treer, T., Habeković, D., Safner, R., Aničić, I., Kolak, A. (1998): Growth of pike (Esox lucius L.) in Croatian reservoir Kruščica. Ribarstvo, 56, 3, 85-90.

Treer, T., Habeković, D., Safner, R., Kolak, A., Aničić, I. (1999): Length-mass relationship in chub (Leuciscus cephalus) from five Croatian rivers. Poljoprivredna znanstvena smotra, 64, 2, 137-142.

Treer, T., Matulić, D., Bogdanović, G., Aničić, I., Safner, R., Piria, M., Šprem, N., Tomljenović, T. (2011): The condition of allochtonous fishes in the Mediterranean Vransko Lake. Journal of Applied Ichthyology, 27, 3, 965-967.

Treer, T., Opačak, A., Aničić, I., Safner, R., Piria, M., Odak, 
T. (2003): Growth of bream, Abramis brama, in the Croatian section of the Danube. Czech Journal of Animal Science, 48, 6, 251-256.

Treer, T., Piria, M., Aničić, I., Safner, R., Tomljanović, T. (2006): Diet and growth of spirlin, Alburnoides bipunctatus in barbel zone of the Sava river. Folia Zoologica, 55, 1, 97-106.

Treer, T., Piria, M., Šprem, N. (2009): The relationship between condition and form factors of freshwater fishes of Croatia. Journal of Applied Ichthyology, 25, 5, 608-610.

Treer, T., Safner, R., Aničić, I., Lovrinov, M. (1995): Fisheries. Nakladni zavod Globus, Zagreb, 464pp. (in Croatian)

Treer, T., Safner, R., Aničić, I., Kolak, A., Dražić, M. (2000a): Morphological variation among four strains of common carp Cyprinus carpio in Croatia. Folia Zoologica, 49, 1, 69-74.

Treer, T., Safner, R., Aničić, I., Piria, M., Odak, T. (2002): The introduction of the fish from the Danube area into the Mediterranean Vransko Lake, Croatia. Book of abstracts of Symposium on Inland fisheries management and the aquatic enviroment. The effects of fisheries management on freshwater ecosystems. Symposium on Inland fisheries management and the aquatic enviroment. The effects of fisheries management on freshwater ecosystems, Windermere (England), June 2002. FAO, Rome, pp. 43.

Treer, T., Safner, R., Ržaničanin, B. (1984): Comparison of the allometric growth of roach (Rutilus rubilio, Pisces) on the basis of standard and total body length. Bilten Društva ekologa Bosne i Hercegovine $B, 3,117-120$. (in Croatian)

Treer, T., Šprem, N., Piria, M. (2014): Condition of huchen (Hucho hucho Linnaeus, 1758) from the CroatianSlovenian Kupa River. Journal of Applied Ichthyology, 30, 1, 168-171.

Treer, T., Šprem, N., Torcu-Koc, H., Sun, Y., Piria, M. (2008): Length-weight relationships of freshwater fishes of Croatia. Journal of Applied Ichthyology, 24, 626-628.

Treer, T., Varga, B., Safner, R., Aničić, I., Piria, M., Odak, T. (2003): Growth of the common carp (Cyprinus carpio) introduced onto the Mediterranean Vransko lake. Journal of Applied Ichthyology, 19, 6, 383-386.

Treer, T., Habeković, D., Aničić, I., Safner, R., Piria, M. (2000b): Salmonid species and their breeding. Agriculturae Conspectus Scientificus, 65, 3, 175-180. (in Croatian)

Trewavas, E. (1971): The type-species of the genera Phoxinellus, Pseudophoxinus and Paraphoxinus (Pisces, Cyprinidae). Bulletin of the British Museum (Natural History), Zoology, 21, 359-361.

Trgovčević, L. (1905a): Paraphoxinus Blkr. and Telestes Bonap. in the waters of Lika and Krbava. Nastavni vjesnik, 2, 87-89. (in Croatian)

Trgovčević, L. (1905b): Paraphoxinus Blkr. and Telestes Bonap. in the waters of Lika and Krbava. Nastavni vjesnik, 3, 159-165. (in Croatian)

Trgovčević, L. (1905c): Paraphoxinus Blkr. and Telestes Bonap. in the waters of Lika and Krbava. Nastavni vjesnik, 4, 247-254. (in Croatian)

Trgovčević, L. (1905d): Paraphoxinus Blkr. and Telestes Bonap. in the waters of Lika and Krbava. Nastavni vjesnik, 5, 331-335. (in Croatian)

Trgovčević, L. (1908): A contribution to the ichthyofauna of the waters of Lika. Glasnik Hrvatskog naravoslovnoga društva, 20, 229-239. (in Croatian)

Trgovčević, L. (1930): Sports fishing opportunities in Lika. Ribarski vjesnik, 7, 11-12, 43. (in Croatian)

Trgovčević, L. (1932): Lika minnows. In; Thaller, Z (ed), Vode i ribe Jugoslavije u slici i riječi. Tipografija, Zagreb, 73-74. (in Croatian)

Trgovčić, K. (2010): Taxonomic traits of the genus Phoxinus in Croatia. Master's thesis, University of Zagreb. (in Croatian)

Trois, E. F. (1896): Sopra un esemplare di Acipenser stellatus proveniente dall' Adriatico. Atti del Reale Istituto veneto di scienze, lettere ed arti, 7, 7, 11751177. (in Italian)

Trojanović, S. (1934): On freshwater fish. Priroda, 24, 1, 13-19. (in Croatian)

Trožić-Borovac, S. (2002): The nutrition of a brown trout Salmo trutta m. fario L in the River Una. Ribarstvo, 60, 3, 83-104. (in Bosnian with English abstract)

Trožić-Borovac, S., Škrijelj, R., Mitrašinović-Brulić, M., Muhamedagić, S., Hamzić, A., Đug, S., Šljuka, S. (2013): Diet and condition coefficient of the grayling (Thymallus thymallus L.) from the Una River basin. Radovi Poljoprivredno-prehrambenog fakulteta Univerziteta $\mathrm{u}$ Sarajevu, 63, 1, 1-10. (in Bosnian)

Tsigenopoulos, C. S., Berrebi, P. (2000): Molecular phylogeny of north Mediterranean freshwater barbs (genus Barbus: Cyprinidae) inferred from cytochrome b sequences: biogeographic and systematic implications. Molecular Phylogenetics and Evolution, 14, 2, 165-179.

Turk, M., Debeljak, Lj. (1995): Economic feasability of the culture of consumption fish under different feeding conditions. Ribarstvo, 53, 4, 141-149. (in Croatian with English abstract)

Turk, M., Debeljak, Lj., Fašaić, K. (1987): Results of rearing herbivorous fish in the fish farms of Croatia in the period 1977 to 1986. Ribarstvo Jugoslavije, 42, 6, 121129. (in Croatian)

Tutman, I. (1948): Eastern mosquito fish. Priroda, 35, 5, 22-24. (in Croatian)

Tutman, P., Freyhof, J., Dulčić, J., Glamuzina, B., Geiger, M. (2017): Lampetra soljani, a new brook lamprey from the southern Adriatic Sea basin (Petromyzontiformes: Petromyzontidae). Zootaxa, 4273, 4, 531-548.

Tutman, P., Glamuzina, B., Bartulović, V., Buntić, I. (2002): Endemic ichthyofauna of the Hutovo Blato wetland. Završni simpozij LIFE Projekta LIFETCY 99/BIH/035 „Nova politika gospodarenja vlažnim područjima 
Hutova Blata“. Ministarstvo graditeljstva, prostornog uređenja i zaštite okoliša HNŽ-K, Mostar, pp. 45-48. (in Croatian)

Tutman, P., Glamuzina, B., Bartulović, V., Dulčić, J. (2007): A new maximum length for Anguilla anguilla (Anguillidae). Cybium, 31, 4, 485-486.

Tutman, P., Glamuzina, B., Dulčić, J. (2008a): Threatened fishes of the world: Salmo dentex (Heckel 1852) (Salmonidae). Environmental Biology of Fishes, 82, 2, 175-176.

Tutman, P., Glamuzina, B., Dulčić, J. (2008b): Threatened fishes of the world: Chondrostoma knerii Heckel, 1843 (Cyprinidae). Environmental Biology of Fishes, 83, 2, 209-210.

Tutman, P., Skaramuca, B., Dulčić, J., Matić-Skoko, S., Kraljević, M., Bartulović, V., Glamuzina, B., Glavić, N., Kožul, V., Antolović, N. (2010): Settlement and recruitment of young marine fish within the broader area of the Neretva Delta. In: Glamuzina, B., Dulčić, J. (eds), Ribe i Ribarstvo, rijeke Neretve. Međunarodni znanstveno-stručni skup "Ribe i Ribarstvo, rijeke Neretve: stanje i perspektive" Opuzen (Hrvatska), 2009. Sveučilište u Dubrovniku i Dubrovačko neretvanska županija, Dubrovnik, 39-58. (in Croatian)

Tutman, P., Hamzić, A., Hasković, E., Dulčić, J., Pavličević, J., Glamuzina, B. (2016): Neretva rudd, Scardinius plotizza Heckel \& Kner, 1858 (Cyprinidae), endemic fish species of the Adriatic watershed; biological-ecological and conservation traits. Annales, Series Historia Naturalis, 26, 2, 185-190.

Tvrtković, N. (1985): The Fish of Yugoslavia. Hrvatski narodni zoološki muzej, Zagreb, 27pp. (in Croatian)

Tvrtković, N. (1998): Vertebrate fauna of the lower Neretva River. Časopis za književnost i znanost, 9, 4, 260-270. (in Croatian)

Tvrtković, N. (2010): Endangered flora and fauna of the City of Zagreb. Hrvatski prirodoslovni muzej, Zagreb, 72pp. (in Croatian)

Tvrtković, N., Franičević, M. (2002): Pisces and lampreys. An overview of the cave and interstitial biota of Croatia. Natura Croatica, 11, Suplement 1, 42-44.

Tvrtković, N., Šašić Kljajo, M., Holcer, D., Grbac, I, Mihoci, I., Pavlinić, I., Štamol, V., Vuković, M., Radović, D., Dumbović, V. (2009): Mapping the fauna of Dalmatia. Priority areas: island of Pag, Krka River estuary, island of Vis and remote islands, island of Mljet, Cetina River. COAST projekt, Split, 140pp. (in Croatian)

Tvrtković, N., Veen, P. (2006): The Dinaric Alps: Rare Habitats and Species. A Nature Conservation Project in Croatia. Part A, Hrvatski prirodoslovni muzej (CNHM): and Royal Dutch Society for Nature Conservation (KNNV), Zagreb, 67pp.

Uzelac, Z. (1952a): Odra, sport fishing area. Ribarstvo Jugoslavije, 7, 4, 58-59. (in Croatian)

Uzelac, Z. (1952b): Softmouth trout (Trutta obtusirostris). Ribič, 3-4, 33-36. (in Croatian)
Valić, D. (2006): Morphological and cytogenetical characteristics of fish from river Krka. Master's thesis, Faculty of Science, University of Zagreb. (in Croatian with English abstract)

Valić, D. (2010): Phylogeny of subfamily Leuciscinae (Teleostei: Cyprinidae) inferred from mitochondrial and nuclear gene analysis. PhD thesis, Faculty of Science, University of Zagreb. (in Croatian with English abstract)

Valić, D., Kapetanović, D., Kurtović, B., Teskeredžić, E. (2007): Fish parasites in the Krka River. In: Marguš, D. (ed), Zbornik radova sa simpozija Rijeka Krka i Nacionalni park "Krka": prirodna i kulturna baština, zaštita i održivi razvitak. Rijeka Krka i Nacionalni park "Krka": prirodna i kulturna baština, zaštita i održivi razvitak, Šibenik (Hrvatska), Listopad, 2005. Javna ustanova "Nacionalni park Krka", Šibenik, 1009-1014. (in Croatian)

Valić, D., Kapetanović, D., Zanella, D., Mrakovčić, M., Teskeredžić, E., Besendorfer, V., Rabova, M., Rab, P. (2010): The karyotype and NOR phenotype of Telestes ukliva (Cyprinidae). Folia Zoologica, 59, 2, 169-173.

Valić, D., Mesarić, M., Teskeredžić, E. (2008): Farming of endangered and indigenous freshwater fish. Stočarstvo, 62, 1, 75-77. (in Croatian)

Valić, D., Vardić Smrzlić, I., Kapetanović, D., Teskeredžić, Z., Pleše, B., Teskeredžić, E. (2013): Identification, phylogenetic relationships and a new maximum size of two rudd populations (Scardinius, Cyprinidae) from the Adriatic Sea drainage, Croatia. Biologia, 68, 3, 539-545.

Vanhove, M. P. M., Economou, A. N., Zogaris, S., Larmuseau, M. H. D., Giakoumi, S., Kalogianni, E., Volckaert, F. A. M., Huyse, T. (2012): Phylogenetics and biogeography of the Balkan "sand gobies" (Teleostei: Gobiidae) vulnerable species in need of taxonomic revision. Biological Journal of the Linnean Society, 105, 73-91.

Vardić Smrzlić, I. (2010): Morphologic and genetic characterization of intestinal parasites (Acanthocephala) of freshwater fish from Croatian rivers. PhD thesis, Faculty of Science, University of Zagreb.

Vardić Smrzlić, I., Valić, D., Kapetanović, D., Dragun, Z., Gjurčević, E., Četković, H., Teskeredžić, E. (2013): Molecular characterisation and infection dynamics of Dentitruncus truttae from trout (Salmo trutta and Oncorhynchus mykiss) in Krka River, Croatia. Veterinary Parasitology, 197, 3-4, 604-613.

Vardić, I. (2006): Viral diseases of Salmonides in Croatia. Master's thesis, Faculty of Science, University of Zagreb. (in Croatian with English abstract)

Veljović, P. (1982): Beitrag zum Studium der Ichthyofauna des Save flusses. Internazionale Arbeitsgemeinschaft Donauforschung, Wien, pp. 210-213. (in German)

Veljović, P. (1985): Dietary spectrum of Esox lucius L., Stizostedion lucioperca L. and Silurus glanis L. in the middle course of the Sava River. Ribarstvo Jugoslavije, 40, 2-3, 51-55. (in Serbian)

Vilizzi, L. (2012): The common carp, Cyprinus carpio, in the 
Mediterranean region: Origin, distribution, economic benefits, impacts and management. Fisheries Management and Ecology 19, 93-110.

Vinterhalter, M. (1963): Chub - a sports fish that should be protected in certain waters. Ribarski list, 3-4, 67-69. (in Croatian)

Vinterhalter, M. (1967): Will the herbivorous fish grass carp and silver carp become new residents in our waters?. Ribarski list, 2, 51-52. (in Croatian)

Vinterhalter, M. (1969): Will the new herbivorous fish species grass carp and silver carp become sports fish?. Ribarski list, 5, 103-104. (in Croatian)

Vlasenko, A. D., Pavlov, A. V., Sokolov, L. I., Vasil'ev, V. P. (1989): Acipenser gueldenstaedti Brandt, 1833. In: Holčík, J. (ed), The Freshwater Fishes of Europe. Vol 1, Part II, General introduction to fishes, Acipenseriformes. AULA-Verlag, Wiesbaden, pp. 294-344.

Vraneš, M. (1966): Threatened Lika minnows. Priroda, 53, 6-7, 186-188. (in Croatian)

Vrebac, M. (1970): Things get better in Brčko. Ribarski list, 4, 86-87. (in Bosnian)

Vrebac, M. (1972): Appeal from the Sava River. Ribarski list, 2, 38. (in Bosnian)

Vrenk, B. (1984): 31 best fishes. Stvarnost, Zagreb, 35pp. (in Croatian)

Vucić, M., Jelić, D., Žutinić, P., Grandjean, F., Jelić, M. (2018): Distribution of Eurasian minnows (Phoxinus: Cypriniformes) in the Western Balkans. Knowledge \& Management of Aquatic Ecosystems, 419, 1-11.

Vucić, M., Sučić, I., Jelić, D. (2017): New distribution data for Alburnus sava Bogutskaya, Zupančič, Jelić, Diripasko \& Naseka, 2017 and Telestes souffia (Risso, 1827) in the western Balkans. Croatian Journal of Fisheries, 4, 161169.

Vukić, J., Kovačić, M., Zogaris, S., Šanda, R. (2016): Rediscovery of Knipowitschia goerneri and its molecular relationships with other European northern Mediterranean Knipowitschia species (Teleostei: Gobiidae). Ichthyological Exploration of Freshwaters, 26, 4, 363-372.

Vukić, J., Ulqini, D., Šanda, R. (2017): Occurrence of Knipowitschia goerneri (Gobiidae) in southern Albania confirmed by molecular tools. Journal of Applied Ichtyhology, 33, 284-290.

Vukosav, P., Mlakar, M., Cukrov, N., Kwokal, Ž., Pižeta, I., Pavlus, N., Špoljarić, I., Vurnek, M., Brozinčević, A., Omanović, D. (2014): Heavy matal contents in water, sediment and fish in a karst aquatic ecosystem of the Plitvice Lakes National Park (Croatia). Environmental Science and Pollution Research, 21, 5, 3826-3839.

Vuković, T. (1961): Population and spawning of Alosa fallax nilotica (Geoffroy) in the waters of the Neretva and Skadar Lake. Godišnjak biološkog instituta Univerziteta u Sarajevu, 14, 85-176. (in Bosnian)

Vuković, T. (1962a): New data on the windering in freshwater and growth of young twaite shad Alosa fallax nilotica in the waters of the lower Neretva. Godišnjak biološkog instituta Univerziteta u Sarajevu, 15, 141-145. (in Bosnian)

Vuković, T. (1963): Fish of Bosnia and Herzegovina. Zavod za izdavanje udžbenika, Sarajevo, 127pp. (in Bosnian)

Vuković, T. (1977): Paraphoxinus alepidotus (Heckel, 1843). New data about distribution in the waters of Yugoslavia. Ribarstvo Jugoslavije, 32, 1, 3-4. (in Bosnian)

Vuković, T. (1982): Systematics of fish. In: Habeković, D. (ed), Slatkovodno Ribarstvo. Ribozajednica - Jumena, Zagreb, 99-168. (in Bosnian)

Vuković, T., Aganović, M. (1971): Analysis of the morphological and taxonomic properties of the species Rutilus rutilus Linnaeus (Pisces, Cyprinidae) from several waters of the Black Sea basin. Zbornik referata sa I. simpozijuma sistematičara Jugoslavije, 207-213. (in Bosnian)

Vuković, T., Ivanišević, B. (1962): Existence of two morphologically different populations of Scardinius erythrophthalmus scardafa (Bonaparte) in the lower Neretva River and Skadar Lake. Godišnjak biološkog instituta Univerziteta u Sarajevu 15, 137-140. (in Bosnian)

Vuković, T., Ivanović, B. (1971): Freshwater Fish of Yugoslavia. Zemaljski muzej BiH, Sarajevo, 268pp. (in Bosnian)

Vuković, T., Kosorić, Đ. (1967): Variations in the length of the dorsal spines of Gasterosteus acculeatus Linne (threespined stickleback in the Buna River. Ribarstvo Jugoslavije, 22, 1, 7-9. (in Bosnian)

Vuković, T., Kosorić, Đ. (1978): Effects of the introduction of fish species in the waters of Yugoslavia and possible impacts on the reconstruction of the ichthyofauna. Ribarstvo Jugoslavije, 33, 4, 92-95. (in Bosnian)

Vuković, T., Prolić, F. (1966): Beitrag zu den taxonomischen merkmalen von Gasterosteus aculeatus aus dem unterlauf der Neretva. Glasnik Zemaljskog muzeja Bosne i Hercegovine u Sarajevu, 5, 175-178. (in German)

Vuković, T., Seratlić-Savić, D., Karanac, V. (1970): Some morphological characteristics of the hybrid Chondrostoma phoxinus (Heckel) $\mathrm{x}$ Paraphoxinus alepidotus (Heckel). Ichthyologia, 2, 1, 155-169. (in Bosnian)

Vuković, T., Vuković, N. (1968): Some taxonomic characteristics of the species Rutilus rubilio (Bonap.): [Pisces, Cyprinidae] from the waters of Yugoslavia and northern Italy. Godišnjak Biološkog instituta Univerziteta u Sarajevu 21, 129-135. (in Bosnian)

Vuletić, S. (1960): Brušani fish farm near Gospić. Ribarstvo Jugoslavije, 15, 2, 42-44. (in Bosnian)

Vutskits, G. (1918): Classis Pisces. Fauna Regni Hungariae, Pars I. Vertebrata. Regia Societas Scientarium Naturalium Hungarica, Budapest, 42pp. (in Latin)

Wanzenböck, J. (1995): Current knowledge on the European mudminnow, Umbra krameri Walbaum, 1792 
(Pisces: Umbridae). Annalen des Naturhistorischen Museums in Wien, 97, B, 439-449.

Wettstein, O. R. (1928): Beiträge zur Wirbeltierfauna der kroatischen Gebirge. Annalen des Naturhistorischen Museums in Wien, 42, 1-45. (in German)

Winterhalter, M. (1964): Pike is pike. Priroda, 51, 3, 75-77. (in Croatian)

Witkowski, A., Bajić, A., Treer, T., Hegediš, A., Marić, S., Šprem, N., Piria, M., Kapusta, A. (2013): Past and present of and perspectives for the Danube huchen, Hucho hucho (L.), in the Danube basin. Archives of Polish Fisheries, 21, 3, 129-142.

Zagorac (1897): First fishing on the Božjakovina estate. Lovačko-ribarski viestnik, 6, 145. (in Croatian)

Zanandrea, G. (1958): Le lamprede dei musei di Zagabria e Lubiana. Biološki glasnik, 11, 45-54. (in Italian)

Zanandrea, G. (1959): Lamprede parassite e non parassite nel bacino Danubio e la nuova entità sistematica. Archivio Zoologico Italiano, 44, 215-250. (in Italian)

Zanella, D. (2003): Biological and morphological characteristics of the subspecies Leuciscus souffia muticellus (Bonaparte, 1837), (Pisces, Cyprinidae). Master's thesis, Faculty of Science, University of Zagreb. (in Croatian with English abstract)

Zanella, D., Mihaljević, Z., Mrakovčić, M., Ćaleta, M. (2009a): Ecology and diet of the endemic Telestes ukliva (Cyprinidae) in the Cetina river System, Croatia. Cybium, 33, 2, 97-105.

Zanella, D., Mrakovčić, M., Mustafić, P., Ćaleta, M., Marčić, Z. (2009b): Threatened fishes of the world: Cobitis narentana Karaman, 1928 (Cobitidae). Environmental Biology of Fishes, 86, 523-524.

Zanella, D., Mrakovčić, M., Mustafić, P., Ćaleta, M. (2008a): Recovery of Telestes ukliva, an endemic species from the Cetina River, Croatia (Cypriniformes, Cyprinidae). Journal of Fish Biology, 73, 311-316.

Zanella, D., Mrakovčić, M., Mustafić, P., Ćaleta, M., Buj, I., Marčić, Z., Zrnčić, S., Razlog-Grlica, J. (2008b): Age and growth of Sabanejewia balcanica in the Rijeka River, central Croatia. Folia Zoologica, 57, 1-2, 162-167.

Zanella, D., Mrakovčić, M., Schneider, D., Mustafić, P., Ćaleta, M., Radić, I. (2003): Growth of Cobitis narentana Karaman, 1928 in the Neretva River, Croatia. Folia Biologica (Krakow), 51 (Supplement), 155-157.

Zanella, D., Mrakovčić, M., Zanella, L. N., Miletić, M., Mustafić, P., Ćaleta, M., Marčić, Z. (2011): Reproductive biology of the freshwater goby Knipowitschia croatica Mrakovčić, Kerovec, Mišetić \& Schneider 1996 (Actinopterygii, Gobiidae). Journal of Applied Ichthyology, 27, 5, 1242-1248.

Zanella, D., Schneider, D., Mrakovčić, M., Mustafić, P., Suić, J. (2000): Ichthyofauna of the Wetland Lonjsko Polje. In: Horvatić, J. (ed), Limnological reports, proceedings. Faculty of Education, Josip Juraj Strossmayer University of Osijek, Osijek, pp. 323-328.

Zanella, L. N. (2009): Variability and morphological traits of Gasterosteus aculeatus (Linnaeus, 1758) in Croatia. Master's thesis, Faculty of Science, University of Zagreb.

Zanella, L. N., Zanella, D., Mrakovčić, M., Miletić, M., Mustafić, P., Ćaleta, M. (2009c): Occurrence of fourspined Gasterosteus aculeatus in an isolated Croatian river population. Journal of Fish Biology, 75, 8, 20522061.

Zanella, L. N., DeFaveri, J., Zanella, D., Merilä, J., Šanda, R., Mrakovčić, M. (2015): Does predation drive morphological differentiation among Adriatic populations of the three-spined stickleback? Biological Journal of the Linnean Society, 115, 219-240.

Zaplata, R. (1932): Cactus roach (Leuciscus virgo Heckel). Ribarski list, 7, 9-10, 102-104. (in Croatian)

Zelić, J., Crnjac, T., Grizelj-Paulić, M. (2011): Sovsko Lake - the "Blue eye" of the Dilj mountain. Hrvatska vodoprivreda, 197, 88-91. (in Croatian)

Zerunian, S. (2004): Pesci delle acque interne d'Italia. Ministero Dell'ambiente; Istituto nazionale per la fauna selvatica „Alessandro Ghigi“, Roma, 257pp. (in Italian)

Zlatović, S. (1890): Report on the Bosna for the year 1640 by Father Paul from Rovinj. Starine, 23, 1-38. (in Croatian)

Zobundžija, V. (1968): Fish species of the Bosut River. Biološki list, 21, 23-80. (in Croatian)

Zrnčić, S., Oraić, D., Ćaleta, M., Mihaljević, Ž., Zanella, D., Bilandžić, N. (2013): Biomonitoring of heavy metals in fish from the Danube River. Environmental Monitoring and Assessment, 185, 2, 1189-1198.

Zrnčić, S., Oraić, D., Mihaljević, Ž., Ćaleta, M., Zanella, D., Jelić, D., Jelić, M. (2009a): First observation of Posthodiplostomum cuticola (Nordmann, 1832): metacercariae in cypriniformes from Croatia. Helminthologia, 46, 2, 112-116.

Zrnčić, S., Oraić, D., Šoštarić, B., Ćaleta, M., Buj, I., Zanella, D., Šurmanović, D. (2009b): Occurrence of parasites in Cobitidae from Croatian rivers draining into two different watersheds Journal of Applied Ichthyology, 25, 447-450.

Zuliani, T., Vidmar, J., Drinčić, A., Ščančar, J., Horvat, M., Nečemer, M., Piria, M., Simonović, P., Paunović, M., Milačič, R. (2019): Potentially toxic elements in muscle tissue of different fish species from the Sava River and risk assessment for consumers. Science of the Total Environment, 650, 958-969.

Zupančič, P. (1990): Cyprinids of the Dinaric karst. Proteus, 52, 218-222. (in Slovenian)

Zupančič, P. (2008): Rare and threatened freshwater fish of the Adriatic basin of Croatia, Slovenia and Bosnia and Herzegovina. Narodna in univerzitetna knjižnica, Ljubljana, 79pp. (in Slovenian)

Zupančič, P., Bogutskaya, N. G. (2000): Description of a new species, Phoxinellus dalmaticus (Cyprinidae: Leuciscine), from the Čikola river in the Krka river system, Adriatic basin (Croatia). Natura Croatica, 9, 2, $67-81$. 
Zupančič, P., Bogutskaya N. G. (2002): Description of two new species, Phoxinellus krbavensis and P. jadovensis, re-description of $P$. fontinalis Karaman, 1972, and a discussion of the distribution of Phoxinellus species (Teleostei: Cyprinidae) in Croatia and BosniaHerzegovina. Natura Croatica, 11, 4, 411-437.

Zupančič, P., Marić D, Naseka, A. M., Bogutskaya, N. G. (2010a): Squalius platyceps, a new species of fish (Actinopterygii: Cyprinidae): from the Skadar Lake basin. Zoosystematica Rossica, 19, 1, 154-167.

Zupančič, P., Mrakovčić, M., Marčić, Z., Naseka, A. M., Bogutskaya, N. G. (2010b): Identity of Squalius (Actinopterygii, Cyprinidae) from Istra Peninsula in Croatia (Adritic Sea basin). ZooKeys, 53, 45-58.
Žikić, R., Bertoša, N. (1980): Influence of water pollution in Pazin on the biocenosis of the lower course of the Pazinčica in 1978. Ichthyologia, 12, 2, 97-108. (in Croatian)

Žutinić, P., Jelić, D., Jelić, M., Buj, I. (2014): A contribution to understanding the ecology of the large spot barbel - sexual dimorphism, growth and population structure of Barbus balcanicus (Actinopterygii, Cyprinidae): in Central Croatia. North-Western Journal of Zoology, 10, 1, 158-166. 